\title{
Novel Insights for Improvement of Tacrolimus Therapy
}

Citation for published version (APA):

Stifft, F. (2020). Novel Insights for Improvement of Tacrolimus Therapy. [Doctoral Thesis, Maastricht University]. Maastricht University. https://doi.org/10.26481/dis.20200703fs

Document status and date:

Published: 01/01/2020

DOI:

$10.26481 /$ dis.20200703fs

Document Version:

Publisher's PDF, also known as Version of record

\section{Please check the document version of this publication:}

- A submitted manuscript is the version of the article upon submission and before peer-review. There can be important differences between the submitted version and the official published version of record.

People interested in the research are advised to contact the author for the final version of the publication, or visit the DOI to the publisher's website.

- The final author version and the galley proof are versions of the publication after peer review.

- The final published version features the final layout of the paper including the volume, issue and page numbers.

Link to publication

\footnotetext{
General rights rights.

- You may freely distribute the URL identifying the publication in the public portal. please follow below link for the End User Agreement:

www.umlib.nl/taverne-license

Take down policy

If you believe that this document breaches copyright please contact us at:

repository@maastrichtuniversity.nl

providing details and we will investigate your claim.
}

Copyright and moral rights for the publications made accessible in the public portal are retained by the authors and/or other copyright owners and it is a condition of accessing publications that users recognise and abide by the legal requirements associated with these

- Users may download and print one copy of any publication from the public portal for the purpose of private study or research.

- You may not further distribute the material or use it for any profit-making activity or commercial gain

If the publication is distributed under the terms of Article $25 \mathrm{fa}$ of the Dutch Copyright Act, indicated by the "Taverne" license above, 
Novel Insights for Improvement

$$
\text { of }
$$

\section{Tacrolimus Therapy}


(C) Frank Stifft, Maastricht, 2020

Cover design: Jean Scheijen | vierdrie.nl Layout: Tiny Wouters

Production: Drukkerij Walters Maastricht BV

ISBN/EAN: 978-94-92741-36-3

Publication of this thesis was financially supported by Alfasigma Nederland BV. 


\title{
Novel Insights for Improvement of \\ Tacrolimus Therapy
}

\author{
PROEFSCHRIFT
}

ter verkrijging van de graad van doctor aan de Universiteit Maastricht, op gezag van de Rector Magnificus, Prof. dr. Rianne M. Letschert, volgens het besluit van het College van Decanen,

in het openbaar te verdedigen

op vrijdag 3 juli 2020 om 12.00 uur

door

Frank Stifft

Geboren op 14 augustus 1970 te Goch, Duitsland 


\section{Promotor}

Prof. dr. J.P. van Hooff

\section{Co-promotor}

Dr. M.H.L. Christiaans

\section{Beoordelingscommissie}

Prof. dr. G.W. Schurink (voorzitter)

Prof. dr. T. van Gelder, Erasmus MC Rotterdam;

Prof. dr. D.R.J. Kuypers, UZ Leuven, België

Prof. dr. H.C. Schouten 


\section{Contents}

$\begin{array}{lll}\text { Chapter 1. Introduction and aims of the thesis } & 7\end{array}$

Chapter 2. Increase in tacrolimus exposure after steroid tapering is influenced 39 by Cyp3A5 and Pregnane $X$ receptor genetic polymorphisms in renal transplant recipients

Chapter 3. Rectal and sublingual administration of tacrolimus: a single dose pharmacokinetic study in healthy volunteers

Chapter 4. A limited sampling strategy to estimate exposure of once-daily modified release tacrolimus in renal transplant recipients using linear regression analysis and comparison with Bayesian population pharmacokinetics in different cohorts

Chapter 5. Effect of breakfast on the exposure of the once-daily tacrolimus formulation in stable kidney transplant recipients

Chapter 6. Lower variability in 24-hour exposure during once-daily compared with twice-daily tacrolimus formulation in kidney transplantation

Chapter 7. General discussion and summary

Chapter 8. Nederlandse samenvatting

Chapter 9. Valorisation .

Addenda

Dankwoord 



\section{Chapter 1}

Introduction and aims of the thesis 



\subsection{Kidney transplantation and immunosuppression}

Chronic kidney disease is a major burden to public health. It often comes with comorbidity and an increased risk for cardiovascular disease. ${ }^{1}$ People may progress to end stage renal disease and renal replacement therapy is warranted. One of the renal replacement modalities is kidney transplantation. There is, however, a shortage in kidney donor organs. In 2017, the Dutch organ transplantation registry reported 1301 applications for waitlist registration with an average time on dialysis until transplantation of 3,1 years. In this year, 979 kidney transplantation were performed, $56 \%$ after living donation. Whilst waitlisted, 82 patients had died, and 104 patients experienced a deterioration of their clinical condition resulting in non-eligibility for kidney transplantation. ${ }^{2}$ Compared to waitlisted dialysis patients, however, standardcriteria kidney transplantation provides a long term survival benefit after the first post-transplant year. ${ }^{3}$ In the Netherlands, this survival benefit is also given for donation after cardiac death. ${ }^{4}$

To ensure survival of the allogeneic kidney, up until now, immunosuppressive therapy is required to prevent allograft rejection. The first kidney was transplanted in 1954 in genetically identical twins and thus without need for any immunosuppression. ${ }^{5}$ The prevention of acute rejection in the first allogeneic kidney transplantations was achieved by total body irradiation at a high cost of fatal (infectious) complications. This era was followed by immunosuppression with the combination of azathioprine, steroids and sometimes anti lymphocyte therapy. Nevertheless, rejection and rerejection rates were high, especially within the first year after transplantation, mounting up to $100 \%{ }^{6,7}$

The rejection rate improved with the venture of cyclosporine, a calcineurin inhibitor (CNI), with a rejection rate up to $40 \%{ }^{6,8}$ increasing one-year graft survival rates to more than $80 \% .^{9}$ In the 1990 's tacrolimus was clinically introduced as a new CNI. It was isolated in 1984 from a type of soil bacterium, Streptomyces tsukubaensis. The name of the drug tacrolimus is derived from 'Tsukuba macrolide immunosuppression' ${ }^{10}$ Compared to cyclosporine, it showed less acute rejection episodes with reduced glucocorticoid requirement. ${ }^{8,11,12}$ Over the years, tacrolimus has evolved to be the cornerstone maintenance immunosuppressant in the field of solid organ transplantation. Between 1995 and 2004, the use of tacrolimus after kidney and liver transplantation increased from $<10 \%$ to $72 \%$ of patients and from $\approx 40 \%$ to $89 \%$ of patients, respectively. ${ }^{13}$ It has been marketed globally since 1993 as "Prograf ${ }^{\circledR "}$ (in the Netherlands "Prograft ${ }^{\circledR}$ "). The tacrolimus capsules are usually taken orally twice daily (BID) but also an intravenous (i.v.) solution is available for cases in which this is not feasible. Since April 2007, a modified release once-daily (QD) formulation of tacrolimus (registered under the trade name Advagraf ${ }^{\circledR}$ ) has become available with a similar efficacy and safety profile compared with the BID 
formulation. ${ }^{14}$ In this thesis, the BID formulation refers to Prograf ${ }^{\circledR}$ and the QD formulation refers to Advagraf ${ }^{\circledR}$ and will be named Tac BID and Tac QD, respectively. US registry data showed that whereas short-term graft survival had significantly improved in the past, long term graft survival had not. ${ }^{15,16}$ In more recent years, apart from short-term outcome, also long term graft survival has improved. In Europe, during the 2000-2015 period improvement of graft survival was even greater in the long than in the short term. ${ }^{17}$ The most common cause for a poorer long-term graft survival is chronic allograft dysfunction. There are several factors contributing to the long-term sequelae of graft loss due to chronic allograft dysfunction. These factors can be non-immune mediated (e.g. ascending urinary tract infections, recurrent renal disease, hypertension, CNI nephrotoxicity), as well as immunologically mediated. ${ }^{18}$ It has been reported that $50 \%$ of the late failing transplant grafts were attributed to chronic antibody mediated rejection (ABMR). ${ }^{19}$ Formation of de novo donor specific antibodies (dnDSA) has been suggested to be one of the mechanisms leading to such an immunologically mediated graft loss. ${ }^{20,21}$ Given the strong relationship between mean tacrolimus levels following dnDSA detection, an association between lower tacrolimus levels $(<5 \mu \mathrm{g} / \mathrm{l})$ and graft loss has been suggested. ${ }^{22,23}$ This may become particularly problematic once tacrolimus levels frequently fall below the suggested level of $5 \mu \mathrm{g} / \mathrm{l}$ in cases of a high variability of $\mathrm{CNI}$ exposure, which has been recognized as an important parameter in poor graft survival. ${ }^{24,25}$ All these factors combined with the high prevalence of tacrolimus use in the field of kidney transplantation implicate the urge for adequate tacrolimus-based therapy. This thesis was, therefore, designed to deliver new insights in pharmacokinetic aspects of tacrolimus therapy, contributing to its optimization and individualization.

\subsection{Tacrolimus: Mechanism of action}

Tacrolimus is a macrolide antibiotic compound and together with cyclosporine belongs to the class of CNI's. The immunosuppressive pathways, however, only in part depend on inhibition of calcineurin. Calcineurin inhibition involves the formation of complexes with immunophilins; tacrolimus binds to FK 506 binding protein 12 (FKBP12). The immunophilin-drug complex interacts with the phosphatase calcineurin (the Calmodulin-Calcium complex), leading to inhibition of the dephosphorylation and translocation of nuclear factor of activated T-cells (NF-AT), a nuclear component thought to initiate gene transcription for the formation of cytokines (such as interleukin-2, gamma interferon). ${ }^{26}$ The net result is the inhibition of T-lymphocyte activation.

It appears that tacrolimus- and cyclosporine-induced immunosuppression is not exclusively due to calcineurin inhibition, i.e. interruption of the NF-AT pathway. ${ }^{27}$ It 
also acts on other pathways of T-cell activation. Within lymphocytes, tacrolimus and cyclosporine also exert immunosuppression through the mitogen-activated protein kinase (MAPK) cascade. The MAPK pathway is a signalling cascade involved in a wide variety of processes, particularly within immune system cells. ${ }^{28}$ Two important arms of the MAPK pathway are the c-Jun N-terminal kinase (JNK or MAPK8) and the p38 (MAPK14) pathways. $^{28}$ They induce the expression of the transcription factor Activating Protein-1 (AP-1), which in turn associates with the NF-AT transcription factor as well as nuclear factor kappa-light-chain-enhancer of activated B cells (NF-kB) to enhance cytokine production, such as IL-2. ${ }^{26}$

Another, calcineurin-independent pathway for immunosuppression concerns the upregulation of the transforming growth factor- $\$ 1$ (TGF- 31 ) ${ }^{29,30}$ TGF- $ß 1$ is a cytokine critical for the regulation of immune system cells. TGF- $\beta$ has been shown to inhibit IL-2-dependent $T$ cell proliferation, as well as exerting a variety of other immunosuppressive effects within $\mathrm{T}$ cells ${ }^{31}$, however, some studies have found evidence showing that neither tacrolimus nor cyclosporine are capable of affecting TGF-ß1 protein or mRNA levels at concentrations in which IL-2 production is successfully inhibited. ${ }^{32}$ Summing up, the impact of the drugs on the transcription of IL-2 is probably the best addressed mechanism, and this particular cytokine plays a large role in the immune response. ${ }^{33}$ The NF-AT dependent mechanisms of action of tacrolimus are summarized in Figure 1.1.

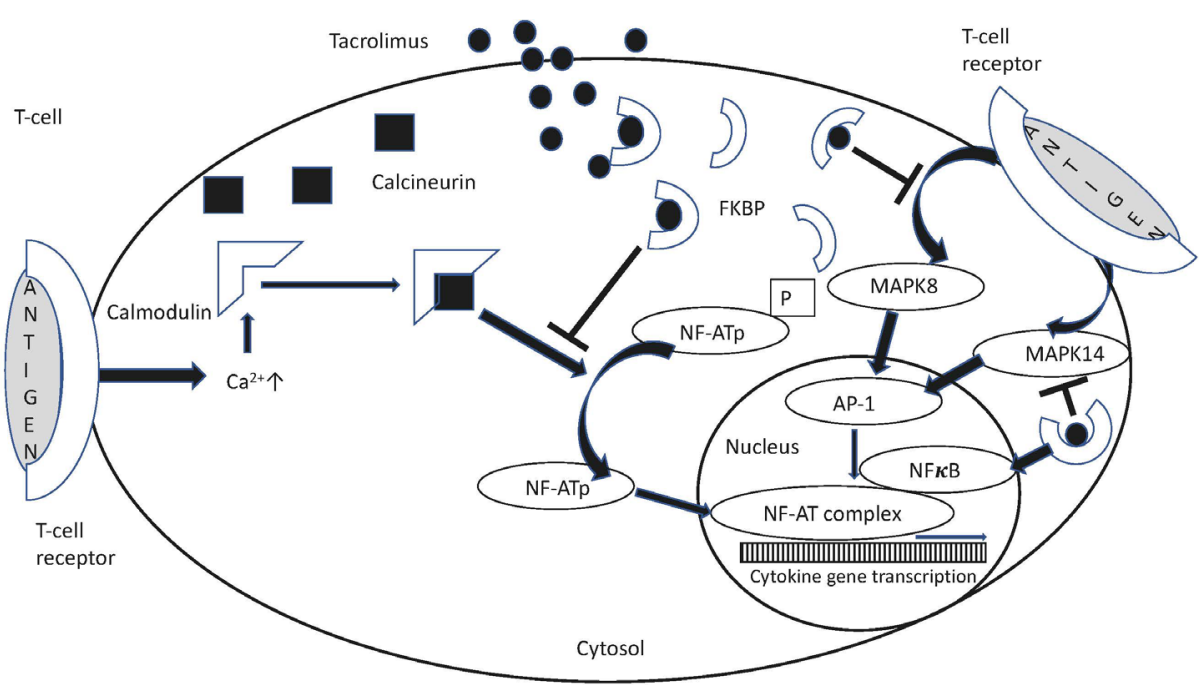

Figure 1.1 Mechanism of action of tacrolimus: see text. Adapted from: Lazarus et al., Drugs Today 2002, 38(1):7. 
Although tacrolimus influences similar pathways like cyclosporine, tacrolimus is more effective in preventing acute rejection, despite its weaker capacity of CNI inhibition. ${ }^{34-36}$ Tacrolimus has also demonstrated efficacy in various types of transplantation as rescue therapy in patients who experience persistent acute rejection (or significant adverse effect's) with cyclosporine-based therapy, whereas cyclosporine has not demonstrated a similar capacity to reverse refractory acute rejection. $^{37,38}$ Compared to cyclosporine, tacrolimus shows less prevalence of hypertension, and dyslipidaemia but a higher rate of Post-Transplantation Diabetes Mellitus after transplantation (PTDM). ${ }^{35,36,39}$ Interestingly, also nephrotoxic side effects were reviewed to be considerably lower in tacrolimus-based treatment compared with cyclosporine. ${ }^{40}$ Other cyclosporine-induced side effects like hirsutism, gum hyperplasia and changes in facial appearance have not been reported under tacrolimus therapy. ${ }^{39}$

It can be concluded from these clinically relevant differences with respect to immunosuppression as well as side effects that other steps distal to calcineurin inhibition in the T-cell activation cascade must be involved. ${ }^{37}$ For example, to a lesser extent, tacrolimus also inhibits B-cell stimulation and antibody production. ${ }^{38}$ Apart from the cytokine gene transcription within the T-lymphocytes, tacrolimus also is described to block cytokine receptor expression and cytokine effects on target cells. ${ }^{41}$ In addition, cyclosporine and tacrolimus displayed different T-cell-receptor beta variable region expression patterns, in vitro. ${ }^{42}$

\subsection{Pharmacokinetics of orally administered Tacrolimus}

\subsubsection{General Pharmacokinetic parameters}

After ingestion of a drug, it is absorbed from the gastrointestinal tract. After a certain lag-time the active compound is detectable in the blood. The time to reach the maximum concentration $\left(T_{\max }\right)$ and the maximum concentration $\left(\mathrm{C}_{\max }\right)$ are parameters that describe the absorption phase of a drug. The drug is then distributed over the body compartments suited for a specific drug and metabolized. This process and the process of elimination will determine the clearance of the drug. The blood concentration before intake of a new dose designates the trough level, which also is the minimum concentration $\left(\mathrm{C}_{0}, \mathrm{C}_{24}\right.$ or $\left.\mathrm{C}_{\mathrm{min}}\right)$. Total exposure to a drug compound is quantified by the area under the concentration time curve (AUC). In case of repetitive dosing, in steady state, the AUC of the blood concentration profile between two dosages is the same as the AUC of single dosing. ${ }^{43}$ The minimal effective concentration (MEC) and the maximal tolerable concentration (MTC) define the therapeutic range. Tacrolimus has a narrow therapeutic range harbouring the risk of allograft loss in case 
of too low and toxicity in case of too high drug levels. General pharmacokinetic parameters are summarized in Figure 1.2.

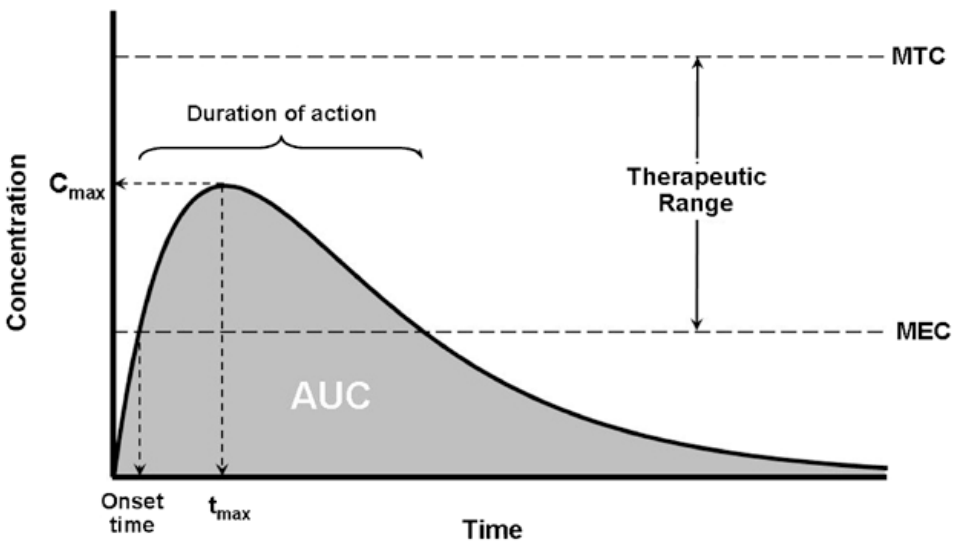

Figure 1.2 Blood concentration time profile with pharmacokinetic parameters. AUC = Area Under the Curve; $C_{\max }=$ maximum concentration, $C_{\min }=$ minimum concentration (trough level), $t_{\max }=$ time to reach $C_{\max }, M E C=$ minimal effective concentration $\mathrm{MTC}=$ maximal tolerable concentration . Source. $^{44}$

\subsubsection{Absorption}

The gut is the first natural barrier for tacrolimus to pass before reaching the systemic circulation. ${ }^{45,46}$ Mucosal enzymes like the Cytochrome P450 (Cyp) 3A and the multidrug efflux pump P-glycoprotein glycoprotein (P-gp; also known as multidrug resistance protein 1 (MDR1) or ATP-binding cassette sub-family $B$ member 1 (ABCB1)), are major contributors to the pre-systemic metabolism of tacrolimus ${ }^{46}$ and present at high levels in the villus tip of enterocytes in the gastrointestinal tract. ${ }^{47}$ Tacrolimus is a substrate of both CYP $3 \mathrm{~A}$ as well as the P-glycoprotein, limiting the absorption of tacrolimus. ${ }^{46}$ The Cyp3A5 appears to be mainly localized in the proximal digestive $\operatorname{tract}^{46,48,49}$ with decreasing catalytic activity downstream. ${ }^{47}$ The P-gp may play a more important role for active extrusion of the absorbed tacrolimus in the rectum, since P-gp mRNA levels are reported to increase longitudinally along the intestine, with highest level in the colon. ${ }^{49,50}$

Since the activity of these enzymes and pumps differ from patient to patient, oral bioavailability of tacrolimus is highly variable and ranges in individual patients from 4 to $93 \%$, on average $25 \%{ }^{51}$ The immediate release (BID) formulation is mainly absorbed in the proximal small intestine (duodenum and jejunum) ${ }^{14}$ the modified release (QD) formulation occurs also in more distal parts of the small intestine by the dissolution properties of the drug (see section on the Tac QD formulation). ${ }^{52}$ After oral administration of Tac BID capsules, peak concentrations $\left(C_{\max }\right)$ are reached after approximately 1.6 to 2.3 hours $\left(T_{\max }\right)$. For Tac $Q D, C_{\max }$ is reached after $2-3$ hours. ${ }^{14}$ 
Normal gastro-intestinal absorption may be impaired under clinical circumstances like vomiting or in cases that the normal swallowing act is not feasible (e.g. in intubated patients). For these circumstances an intravenous solution is available, the practical implementation, however, being very cumbersome due to inappropriate dose conversions and adverse events of the infusion solution. ${ }^{53,54}$ Alternatively, drug absorption may also be achieved through the sublingual or the rectal mucosa. In case of tacrolimus, there are reports on sublingual absorption of the drug, which could not be confirmed in a well-designed study in our centre. ${ }^{43}$ Rectal drug absorption could not only be interesting in a search for a way of alternative tacrolimus administration, but is of special interest since local rectal application of tacrolimus is already performed in patients suffering from refractory distal colitis. ${ }^{55}$ For both sublingual and rectal tacrolimus absorption firm pharmacokinetic data are sparse.

\subsubsection{Distribution}

The plasma protein binding of tacrolimus is approximately $99 \%$ and is independent of concentration over a range of 5-50 $\mu \mathrm{g} / \mathrm{l}$. Tacrolimus is bound mainly to albumin and alpha-1-acid glycoprotein, and has a high level of association with erythrocytes due to a high content of FK-binding proteins in erythrocytes. ${ }^{51}$ Therefore, blood drug concentrations are significantly higher (average 15 times, range 4-114 times) than the corresponding plasma values. ${ }^{56,57}$ Due to the extensive distribution of tacrolimus into the erythrocytes, its apparent volume of distribution based on whole blood concentrations is much lower (1.0-1.5 l/kg) than that based on plasma concentrations (about $30 \mathrm{l} / \mathrm{kg})^{58}$

\subsubsection{Metabolism}

Tacrolimus is extensively metabolised by hydroxylation and demethylation to metabolites. The major metabolite is 13-0-demethyl-tacrolimus with negligible immunosuppressive activity in vitro. Cytochrome P450 3A enzymes in the liver are major enzymes responsible for tacrolimus phase I metabolism leading to a substantial first-pass effect. ${ }^{59,60}$ Since Tac QD (Advagraf) contains the same active constituent as Tac BID (Prograf(t)), it shares the same metabolic pathways. The first pass effect can be circumvented after i.v., sublingual or rectal administration of the drug. This may have pharmacokinetic and pharmacodynamic consequences which, so far, are not well studied in case of tacrolimus.

\subsubsection{Elimination}

Following intravenous and oral administration of 14C-labelled tacrolimus, most of the radioactivity is eliminated in the faeces. ${ }^{61}$ Approximately $2 \%$ of the radioactivity is eliminated in the urine. Less than $1 \%$ of unchanged tacrolimus is detected in the urine 
and faeces, indicating that tacrolimus is almost completely metabolised prior to elimination, bile being the principal route of elimination. The average total body clearance and thus the half-life of tacrolimus is variable. In adult heart, liver and kidney transplant patients, clearances of $3.9 \mathrm{l} / \mathrm{h}, 4.1 \mathrm{l} / \mathrm{h}$ and $6.7 \mathrm{l} / \mathrm{h}$, respectively, have been estimated from whole blood concentrations. ${ }^{14}$ In transplant recipients, the average half-life amounts 12-15 hours. Factors such as low haematocrit and protein levels, which result in an increase of the unbound fraction of tacrolimus, or corticosteroid-induced increased metabolism, are considered to be responsible for the higher clearance rates observed following transplantation. ${ }^{62}$ A summary of the different aspects of tacrolimus disposition is given in Figure 1.3.

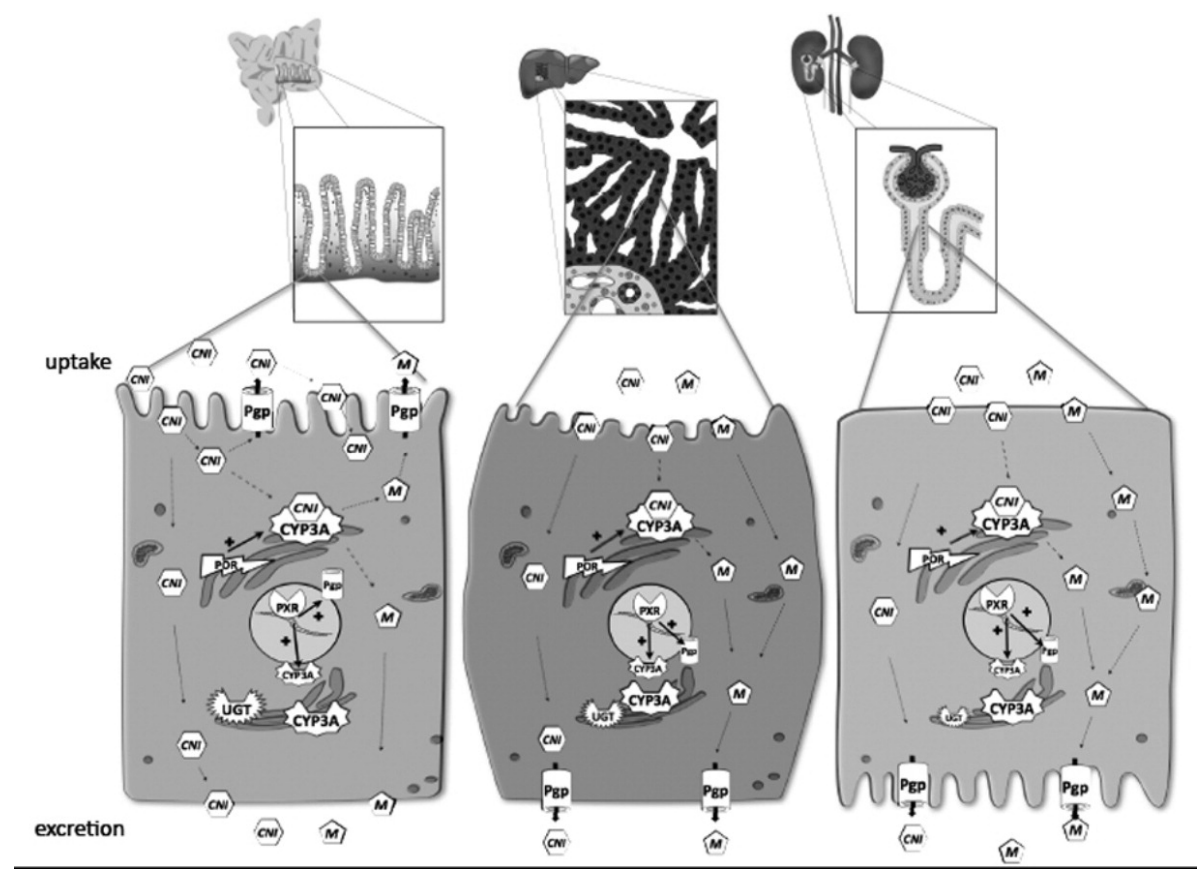

Figure 1.3 Route map of CNI's and CNI metabolites through enterocyte, hepatocyte and proximal tubular cell with the tissue specific cellular localization of enzymes and transporters involved in $\mathrm{CNI}$ metabolism. (M: CNI metabolite). In the intestines the CNI will enter the enterocyte by passive diffusion at the apical cell surface. There it can encounter P-gp and as a result be effluxed back into the intestinal lumen before (or after metabolization) by Cyp3A (and possibly UGT). The drug then has a new opportunity to re-enter the cell, allowing more metabolites to be formed and less of the unchanged drug to pass toward the basolateral surface. The Pregnane $X$ receptor (PXR) and $P 450$ oxidoreductase (POR) are involved in functional Cyp3A and $A B C B 1 / P$-gp expression. In the hepatocyte and proximal tubular cell, the drug enters from the basolateral side and is exposed to metabolization prior to efflux at the apical surface. As a result, less metabolites are formed and more unchanged drug traverses the membrane. Source with permission. ${ }^{45}$ 


\subsection{Narrow therapeutic index and therapeutic drug monitoring}

Considering tacrolimus, there is no simple short term pharmacodynamic surrogate for its therapeutic effect, contrary to other established drugs such as e.g. beta blockers which pharmacodynamically show a decrease of the heart rate after their administration. Therefore, tacrolimus therapy relies on measurement of blood concentrations aiming at a sufficient immunosuppressive effect but the same time avoiding toxic effects in case of over-exposure. The relationship between optimal tacrolimus whole blood concentrations and efficacy or toxicity has not yet been fully established. ${ }^{63}$ Efficacy, in clinical terms, stands for prevention of immunological damage leading to transplant rejection. While the probability of acute rejection decreases with increasing tacrolimus exposure, the chances of toxicity increases, as illustrated in Figure 1.4.

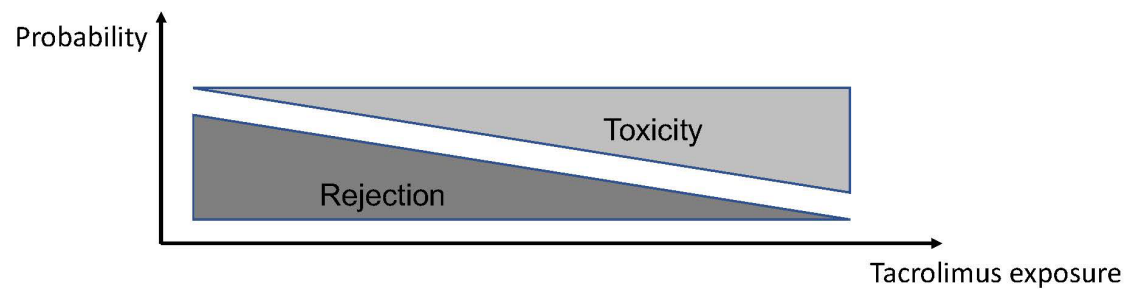

Figure 1.4 The gradual overlap between tacrolimus efficacy and toxicity.

The ratio that compares the blood concentration at which a drug causes a therapeutic effect to the amount that causes death (in animal studies) or toxicity (in human studies) defines the therapeutic index. ${ }^{64}$ For most drugs, the therapeutic window is wide enough, and the $C_{\max }$ of the drug and the AUC achieved when the recommended doses of a drug are prescribed lie sufficiently above the minimum therapeutic concentration and sufficiently below the toxic concentration. ${ }^{65}$ Drugs with a narrow therapeutic index have a narrow window between their effective doses and those at which they produce adverse toxic effects. For tacrolimus, this range is rather small which makes it a 'Narrow Therapeutic Index Drug'.

The definition of a therapeutic and/or toxic effect in clinical practice is highly dependent on the type of therapeutic or toxic effect under consideration and may be influenced by co-medication. ${ }^{68}$ For example, the targeted tacrolimus exposure may be different when co-administered with other immunosuppressive drugs, such as induction therapy, depending on the center-specific immunosuppressive protocol. 
Another complicating factor is the variability of pharmacological response between but also within patients. ${ }^{57,63,69}$

Therefore, tacrolimus therapy is finding the balance within the given narrow therapeutic window and predicting which drug concentration will be achieved with a particular dose change hampered by the high pharmacokinetic variability of tacrolimus. ${ }^{51}$ Given the narrow therapeutic window harbouring the risk of allograft loss in case of too low and toxicity in case of too high drug levels, and given the variability, drug exposure should be monitored by therapeutic drug monitoring (TDM) according to the drug-specific recommendations. ${ }^{70}$

\subsubsection{Tacrolimus exposure targets}

As summarized above, the target tacrolimus exposure depends on the time after transplantation, immunosuppressive co-medication and the anticipated risk of rejection (e.g. donor-recipient HLA-matching). Because evidence-based guidelines are lacking immunosuppressive regimen may be different from center to center. ${ }^{66,68,71,72}$ The tacrolimus exposure is best measured by the AUC, however, in clinical practice, single or several time point whole blood concentrations serve as a surrogate to estimate this. Trough level $\left(C_{\min }\right)$ measurement is the most widely used method ${ }^{63}$, which will be further outlined in paragraph 1.4.2.

There have only been few clinical trials that have specifically related tacrolimus drug concentrations to a clinical outcome, so that the relationship between tacrolimus whole blood concentrations and efficacy or toxicity has not yet been fully established. ${ }^{63}$ Therapeutic ranges developed for tacrolimus have generally been based on clinical/empirical observations, in quite small samples of patients. ${ }^{66}$ In addition, the targeted tacrolimus concentrations change according to the transplantation era with growing experience of tacrolimus therapy: whereas $C_{\min }$ concentrations as high as $20 \mathrm{\mu g} / \mathrm{l}$ were common in the early 1990s, targeted concentrations were reduced after the publication of the Symphony trial in 2007, where trough levels between 3 and $7 \mu \mathrm{g} / \mathrm{l}$ were targeted in combination with administration of daclizumab, mycophenolate mofetil, and corticosteroids. ${ }^{68}$

With this study it had been suggested that treatment for 12 months with a regimen containing "low-dose" tacrolimus combined with daclizumab, mycophenolate mofetil and corticosteroids provided adequate immunosuppression with better renal function and less acute rejection, as well as better allograft survival than did regimens with standard-dose cyclosporine or low-dose sirolimus. ${ }^{68}$ Although low tacrolimus trough levels were targeted (3-7 $\mu \mathrm{g} / \mathrm{l}$ ) during the first year post-transplant, the study patients achieved a higher average trough level of $6-8 \mu \mathrm{g} / \mathrm{l}$ and 30 to $40 \%$ of patients remained above the target. ${ }^{73}$ The achieved average tacrolimus trough level of $7.3 \mu \mathrm{g} / \mathrm{l}$ was still lower than that commonly used in most transplant centres at that time, but was 
above the targeted narrow range, questioning the qualification of "low-dose" tacrolimus treatment but could be regarded as acceptable in clinical practice. ${ }^{74}$

In an earlier study, a significant linear trend towards increasing rates of toxicity with increasing trough concentrations of tacrolimus was observed. Conversely, episodes of acute rejection were associated with lower trough concentrations. Results from this study indicated that trough levels between $5 \mu \mathrm{g} / \mathrm{l}$ and $25 \mu \mathrm{g} / \mathrm{l}$ should be targeted in an attempt to optimize the risk/benefit ratio. $^{75}$ Considering the time after transplantation, during the early post-transplant period the risk of acute rejection has been shown to be higher in patients with low systemic exposure (as expressed by a 12-hour AUC: $\mathrm{AUC}_{0-12}$ ) in a European multicenter trial. ${ }^{76}$ It was concluded that to reduce the risk of acute rejection, a minimum $A_{U C C_{0-12}}$ of $200 \mu \mathrm{g}^{*} \mathrm{~h} / \mathrm{l}$ with a corresponding trough concentration of $10 \mu \mathrm{g} / \mathrm{l}$ should be achieved by day 2 to 3 posttransplant (for which reason, in our centre, tacrolimus is already administered one day before engraftment). On the other hand, the risk of toxicity (tremor, diabetes, nephrotoxicity) has been shown to be significantly higher with prolonged exposure to blood levels $>20 \mu \mathrm{g} / \mathrm{l}^{76}$

The relation of exposure indices with tacrolimus efficacy and side effects has also been described in a well-designed single centre prospective pharmacokinetic study in 100 kidney transplant recipients that have been studied for one year from grafting. It has been shown that at day 7 the highest rejection rates occurred in patients within the lowest quartile of the combined Tac BID and mycophenolate mofetil AUC. This study has provided a conceptual basis for defining a combined target therapeutic window for tacrolimus (and mycophenolate mofetil) based on $\mathrm{AUC}_{0-12}$ measurements. In addition, they have shown that abbreviated sampling for prediction of the AUC was better correlated to clinical endpoints (i.e. infections) than was the pre-dose trough level, indicating the need for adequate monitoring strategies. ${ }^{71}$

The optimal tacrolimus trough level target later post-transplant (i.e. 3 month) to prevent acute rejection of transplanted allografts is unclear. ${ }^{77,78}$ Most institutions reduce tacrolimus exposure between two and three months post-transplant in rejection-free kidney transplant recipients. ${ }^{73}$ The concept of tacrolimus dose reduction was introduced to limit the long-term side effect of CNIs on the renal allograft known as 'chronic CNI nephrotoxicity' as well as other CNI-related side effects of immunosuppressive therapy. ${ }^{36,79}$ However, as already outlined earlier, there is, growing evidence that formation of de novo donor-specific antibodies ( $d n D S A$ ), being associated with antibody mediated rejection (ABMR), is a major contributor to late graft loss. ${ }^{19-21}$ In an observational study, lower tacrolimus trough levels were significantly associated with increased risk of acute rejection in the first 6 months post-transplant with additional risk of acute rejection between months 3-6 posttransplant. ${ }^{73}$ In line with this observation is a threshold tacrolimus level of $<5 \mu \mathrm{g} / \mathrm{I}$ below which an increased rate of dnDSA detection and graft loss has been shown and higher trough levels predicted better graft survival. ${ }^{22,23}$ Therefore, the timing and 
practice of tacrolimus dose reduction were recommended to be personalized based on the individual's risk factors. ${ }^{73}$

\subsubsection{Therapeutic Drug Monitoring (TDM) strategies for tacrolimus}

For tacrolimus the AUC is the golden standard for tacrolimus exposure. ${ }^{63}$ As with any measurement of AUC, this is time consuming, expensive and not convenient for the patient given the number of blood samples that have to be drawn within the defined period. Given the high correlation between the trough level and the $\mathrm{AUC}_{0-12}{ }^{80}$, together with practical considerations, the trough level has become the clinical standard of care as a surrogate for the AUC. However, several studies have questioned the usefulness of the trough level for prediction of outcome, and reported a poor correlation with the AUC. ${ }^{71,81-84}$ Besides, the correlation between these parameters changes with transplantation type and time post-transplant. ${ }^{57,85}$

Consequently, other single time points have been studied and reported to have a better correlation with the AUC. In a study performed by Kuypers et al. the best correlation between a single tacrolimus concentration time point and the full 12-hour AUC of Tac BID was obtained at 6 hours post-administration. ${ }^{71}$ In another study published two years earlier, in 15 stable kidney transplant recipients, $\mathrm{C}_{4}$ had the best correlation with $\mathrm{AUC}_{0-12}$, whereas $\mathrm{C}_{0}$ had the least. ${ }^{82}$ In the same year in yet another study, the 5-hour post-dose sample was most predictive for $\mathrm{AUC}_{0-12 .}{ }^{83}$ Limited sampling strategies (LSS's) comprising two or more blood sampling time points have been proposed, which showed high correlation with and better prediction of $\mathrm{AUC}_{0-12}$. $71,82,83,86,87$

For Tac QD, in multiple studies in stable and de novo transplant recipients the relationship between $\mathrm{C}_{\min }$ and $\mathrm{AUC}_{0-24}$ has been described. ${ }^{88-92}$ In these studies, correlation was consistently reported as moderate to high ( $r>0.80)$, and the slope of the line of best fit was shown to be similar for the two formulations. Subsequently, it has been repeatedly concluded that the TDM used for Tac BID (trough measurement) could be readily applied to the QD formulation. The correlation coefficients reported in the phase II trials were higher than those previously reported in the literature for Tac BID. ${ }^{52}$ It seems reasonable to think, however, that the same difficulties in prediction of the AUC that accounted for Tac BID, are similar for Tac QD. In addition, for a prolonged release formulation other time points than trough levels may give better information on drug exposure.

Besides, different methods for predicting drug exposure to tacrolimus have been described and tested..$^{93}$ In brief, tacrolimus exposure is predicted through either linear regression analysis (LRA) or by Bayesian forecasting in population pharmacokinetic modelling (Maximum A Posterior Bayesian (MAPB) estimation). The advantage of the former would be its easy implementation in daily clinical practice by putting the blood concentration at the designated sampling time points in the regression formula. The 
clear disadvantage would be the need for exact timing of the blood sample. This could be circumvented by Bayesian forecasting. In this instance, however, the clinician would depend on software modelling requiring special expertise as exemplified by a French group. ${ }^{94}$ Whether these two methods differ in their predictive performance is unresolved, so far.

\subsubsection{Dried blood spot sampling}

Therapeutic drug monitoring can be quite cumbersome for patients and care givers. Often patients have to travel from far for their post-transplant outpatient control. In this setting, blood samples are drawn at the transplantation centre early in the morning for measurement of the trough level. Results will not be available by the time they have the appointment, and the care giver will have to contact them in case a dose change is necessary. Sometimes, monitoring strategies with more frequent or later sampling are custom, which, obviously, comes with practical inconveniences.

Dried blood spot sampling (DBS) could be a useful alternative sampling method. With DBS, capillary blood is obtained from a finger prick with an automatic lancet by the patients themselves and the drop of blood is applied to sampling paper. After drying, the paper with the blood spot sample is sent by mail to the laboratory. Possible advantages of therapeutic drug monitoring with DBS are: 1) no phlebotomist is necessary; 2 ) the patients can stay at home; 3 ) monitoring results are known when patients visit the clinician for routine control; 4) monitoring at any desired sampling time point can conveniently be done and an area under the blood concentration-time curve (AUC) can be constructed; and 5) because most analytes are very stable when dry, it is expected that blood spot samples can be stored for a long time at ambient temperature. 95

The method of DBS has been routinely used for measuring tacrolimus levels in our outpatient clinical practice for over a decade. In our institute, Dried Blood Spot analysis of tacrolimus has been developed and validated as being a reliable and practical method for home sampling at any desired time point. ${ }^{95-97}$ This method can easily be taught to patients and they find it convenient. Although this technique is not yet widely applied elsewhere, it could be easily implemented in centres were tacrolimus levels are measured by UPLC-MS/MS. ${ }^{96}$ In a validation study from our institute it was shown that 1) The difference between venous and blood spot sampling of less than $15 \%$, has considered to be clinically acceptable. 2) For results of blood spot sampling with and without nurse assistance comparable results were obtained. 3) There was no significant difference of tacrolimus concentrations before and after postal transport, even over very long distances. Concentration-time curves constructed with the results of both methods did not differ significantly. ${ }^{95}$ In conclusion, the DBS method appears to be a valid method for monitoring tacrolimus concentrations. 


\subsection{Variability of tacrolimus exposure}

One of the immunologically mediated risk factors for graft loss are variable medication trough concentrations. By now, this negative impact of high variability in drug exposure on graft survival has been established in several studies. ${ }^{24,25}$ Variability in drug exposure may result from non-compliance or can be due to pharmacokinetic variability. ${ }^{18}$ Non-compliance (also called non-adherence) is an important factor that contributes to the phenomenon of intrapatient variability, which stresses the need for good communication with and education for the transplant recipient and his or her environment. In a review paper, the reported prevalence of non-adherence varied widely, ranging from 2 to $67 \%$, depending on the used operational definitions, case finding and measurement methods. A weighted mean prevalence, calculated over all studies that measured non-adherence by self-report, was $27.7 \% .{ }^{98}$ It has been concluded from a meta-analysis that the odds of graft failure increased sevenfold in non-adherent subjects compared with adherent subjects. ${ }^{99}$ Given the reported prevalence of non-adherence, one always will have to consider some degree of noncompliance. Simplifying dosing regimen (e.g. once daily dosing) could be beneficial for improving therapy adherence.

Besides non-compliance, other factors also play a role in variability of drug exposure, which, if modifiable, could be additional targets for interventions optimizing chances for graft survival. These factors may rather reflect the pharmacodynamic and the pharmacokinetic properties of the drug, i.e. result from the interaction of patient factors and drug related qualities. In this thesis, the focus will mainly lie on how pharmacokinetic factors can contribute to variability of drug exposure.

\subsubsection{Quantification of variability}

Different patients on the same drug dosage can expose different drug concentrations (inter-patient variability), but also within one individual, different drug concentrations at different time points can exist with unchanged drug dosage (intrapatient variability). There may be consensus about which target levels should be achieved, whereby the inter-patient variability can be handled, however, dosage will still differ from patient to patient. At the same time, a high intrapatient variability poses the real challenge, since the impact of adaptions in current drug dosages to alterations in actual drug exposure will be very hard to predict. So, it can be very difficult for the physician to decide when dosages should be changed and when not, since (trough) levels will be higher or lower anyway, even without dose corrections. In these cases, the average drug exposure may be within acceptable limits but at times either above or below the target level, which puts the patient and the graft at risk.

There are several measures to quantify the intrapatient variability such as the variance, the standard deviation (being the square root of the variance), the Mean 
Absolute Deviation (MAD) and the coefficient of variation (CV), as reviewed elsewhere. ${ }^{100}$ The problem is that using different variability measures impedes direct comparison of reported values. According to the European Medicines Agency (EMEA) on bioavailability and bioequivalence, the $\mathrm{CV}$ can then be calculated as follows ${ }^{101}$ :

$$
C V_{W R}(\%)=100 \sqrt{e^{s_{W R}^{2}}-1}
$$

where $S_{W R}$ is the within subject standard deviation of the natural log-transformed values. ${ }^{101}$ The $\mathrm{CV}$ is a statistical measure for assessment of the degree of variation, which represents the ratio of the standard deviation to the mean. Given the limited number of subjects in many pharmacokinetic studies, the data can be skewed and should be (natural) log-transformed, to get a normal distribution. To comply with the EMEA standard, in this thesis, the intrapatient variability will be quantified by the CV. In many studies, as in our studies discussed in the latter chapters, tacrolimus concentration measurement is only considered in a steady state and mostly during the "stable" phase after transplantation (e.g. at least 6 months after transplantation). ${ }^{100}$ A steady state in this thesis excludes recent changes in tacrolimus dosage, interacting co-medication, states of diarrhoeal and liver diseases.

\subsubsection{Factors contributing to the variability of tacrolimus}

Inter- and intrapatient variability is caused by many factors with a complex interplay covering all the different fields of pharmacokinetics from absorption to distribution and clearance of the drug, respectively. Each of these aspects may be influenced by patient-bound factors considering compliance, comorbidity and co-medication which, on its turn, will be influenced by pharmacogenetics. In addition, factors that are not related to the patient, such as variability in the measurement of the tacrolimus concentration and elapsing time since transplantation play a role in the inter- and intrapatient variability of tacrolimus. In the following paragraphs several of these items will be described.

\subsubsection{Causes for inter-patient variability}

Demographic factors and drug-drug interactions are well-known causes of this interindividual variability in tacrolimus disposition. ${ }^{57,58}$ Paediatric transplant recipients, for example, require two- to fourfold higher doses of tacrolimus to reach target levels. ${ }^{57}$ Adult renal transplant recipients have shown higher tacrolimus clearance compared with liver transplant recipients and healthy volunteers. ${ }^{57}$ Such observations have been partly related to the presence of low haematocrit and albumin concentrations in kidney patients, different liver function and differences in corticosteroid dosage. ${ }^{56}$ In diabetic patients a $38 \%$ reduction in the AUC of tacrolimus was reported compared 
with non-diabetic patients pre-transplant. ${ }^{102}$ This may be due to disturbed gastrointestinal motility in patients with diabetes mellitus.

Differences between ethnic groups may result from racial differences in genes encoding the metabolizing enzymes Cyp3A5 and Cyp3A4, and possibly, the drug transporter ABCB1 (P-glycoprotein; P-gp). ${ }^{93,103-105}$ Patients with the high metabolizing Сур3A5*1 genotype have a higher tacrolimus clearance as expressed by a lower $\mathrm{AUC}_{0}$ ${ }_{24}$ and $C_{0}$ corrected for the dose. ${ }^{106,107}$ Since African American and Asian patients have a higher prevalence of the Cyp3A5*1 genotype, this may be the substrate for higher tacrolimus dosages $(\mathrm{mg} / \mathrm{kg})$ compared with whites to achieve similar concentrations. $^{108}$ Polymorphisms in the Cyp3A5 gene explain $40-50 \%$ of the variability in tacrolimus dose requirement. ${ }^{109,110}$ Genetic polymorphisms of other proteins, like e.g. the Pregnane X-Receptor (PXR) may also explain inter-patient differences in tacrolimus pharmacokinetics. ${ }^{111}$ The PXR will be analyzed in more detail in a later section of this thesis. The role of pharmacogenetics in tacrolimus pharmacokinetics in solid organ transplantation is reviewed in more detail in several papers. $^{104,112-114}$

\subsubsection{Causes for intrapatient variability}

Other factors than those discussed for inter-patient variability can be responsible for the intrapatient variability. Some of which may be modifiable or at least have to be considered when interpreting results of tacrolimus measurement in blood and are summarized in Table 1.1. Some of them will be briefly reviewed in the following sections.

Table 1.1 Modifiable vs. non-modifiable causes for intrapatient variability of tacrolimus exposure.

\begin{tabular}{ll}
\hline Causes for intrapatient variability & \\
\hline Modifiable & Non-modifiable \\
Therapy adherence & Tacrolimus assay \\
Generic substitution & Haematocrit \\
Corticosteroids & Albumin \\
Drug interaction & Diurnal variation \\
Food & Diarrhoeal illness \\
& Liver disease \\
\hline
\end{tabular}

\section{Tacrolimus assay}

One of the factors which have to be considered is the tacrolimus assay. There are several methods available with different lower limits of quantification and inter-assay variability. In the earlier days, measurement of tacrolimus concentration has been mainly performed by an immunoassay technique. The original immunoassay kit was configured to measure tacrolimus concentrations over a range of about 5-40 $\mu \mathrm{g} / \mathrm{l}$, 
reflecting the target ranges in use at that time. As experience with the use of the drug increased, tacrolimus target concentration ranges were reduced and the assay was no longer able to measure the lower concentrations accurately. ${ }^{63}$

Nowadays more laboratories rely on the method of High Performance Liquid Chromatography with tandem Mass Spectrometry (HPLC MS/MS). In the laboratory of the Maastricht University Medical Centre, which participates at the international tacrolimus proficiency testing scheme, the lower limit of quantification is $1.0 \mu \mathrm{g} / \mathrm{l}$. Inter-assay precision (i.e. how much do separate measurements deviate from each other) lies between $4,6 \%$ and $8,2 \%$ for concentrations between 3 and $13 \mu \mathrm{g} / \mathrm{l} .{ }^{95}$ So in our centre, for a patient in the maintenance phase when the targeted trough level lies between 4 and $6 \mu \mathrm{g} / \mathrm{l}$, the results of repeated measurements could deviate by almost $10 \%$ and can thus contribute to inter- as well as intrapatient variability.

\section{Time since transplantation}

It is also important to consider that tacrolimus pharmacokinetics changes according to the time post-transplant. Shortly after transplantation, several clinical factors, like changes in haematocrit and albumin, will result in fluctuating drug concentrations. There is a correlation between relative clearance and both haematocrit and albumin concentration over the first 12 weeks post-transplant. ${ }^{62}$ This is consistent with the strong binding of tacrolimus to red blood cells and serum albumin. Haematocrit and albumin concentrations are generally lower in kidney transplant patients immediately post-surgery and increase significantly as the patient recovers. As tacrolimus concentrations are measured in whole blood, low haematocrit and albumin concentration will result in a reduction in total drug concentration in whole blood, whereas the fraction of unbound tacrolimus increases. ${ }^{57}$

Later on, dose reduction due to lower target trough level with elapsing time after transplantation will result in fluctuating tacrolimus levels, as well. ${ }^{58}$ In addition, a decrease in the dosage of tacrolimus required to maintain similar trough concentrations with increasing time post-transplant has also been reported. ${ }^{71} \mathrm{~A}$ decrease in tacrolimus clearance with time is generally considered to be the reason for this or due to tapering of steroids. ${ }^{57}$ However, also in a more stable condition, several months after transplantation, some patients still show highly variable drug concentrations each time they visit the outpatient clinic. Possible reasons for this will be reviewed in the following sections.

Factors that may cause variable drug concentrations also several months after transplantation may be related to generic substitution of tacrolimus, diurnal variation, co-ingestion of food or changes in co-medication. Since transplantation may come with comorbidity or complications, diarrhoea can contribute to changes in gastrointestinal absorption and the metabolization of the drug can be affected by liver 
disease. $^{57}$ In severe cases the normal oral application of the drug (e.g. in cases of sedation or intubation) is not feasible.

\section{Generic substitution}

For several years generic Tac BID preparations have been available. They are required to fulfil the criteria for bioequivalence with the original formulation (Prograf), i.e. the geometric mean ratio of the AUC for the generic preparation to Prograf had to lie within 80 and $125 \%{ }^{101}$ This was reduced to 90 and $111 \%$ later on, as Prograf was considered a critical narrow therapeutic index drug (see also later section on Tac QD). ${ }^{101}$ However, there is no requirement to demonstrate bioequivalence with the other generic formulations, which may result in variable tacrolimus exposure. ${ }^{100}$ It has been stated that uncontrolled substitutions of different generic drugs poses a theoretical potential danger for the kidney graft. A retrospective reanalysis of existing bioequivalence studies on different drugs (other than tacrolimus) revealed that the differences in exposure observed after switching from a brand-name to a generic drug were comparable with those observed upon repeated administration of the brand-name drug without switching and only depended on the intrasubject variability. ${ }^{115}$ Considering tacrolimus treatment, in clinical practice, TDM will monitor potential changes in drug exposure and doses can consequently be adjusted to ensure equivalent efficacy.

\section{Diurnal variation}

From the pharmacokinetic studies it is well known that the $C_{\max }$ after ingestion of the evening dose of Tac BID is lower compared with the $C_{\max }$ after the morning dose. $^{49,112,113,116,117}$ The reasons for this are not completely understood but may rely on diurnal variation of gastro-intestinal motility, food interaction or diurnal activity of intestinal P-gp and/or Cyp3A.

\section{Drug interaction}

Few formal pharmacokinetic studies investigating tacrolimus drug interactions have been conducted, except for the role of steroids, as will be outlined in the next section. Some potential interactions were derived from animal or in vitro studies, that will not always translate into real clinical significance in the setting of organ transplantation. ${ }^{118,119}$ Pharmacokinetically spoken, as tacrolimus is metabolised by Cyp3A enzymes and is a substrate of the drug efflux pump P-glycoprotein, drugs or concomitant use of certain substances (e.g. St John's wort or grapefruit juice) that inhibit or induce these systems may increase or decrease blood tacrolimus concentrations, respectively. ${ }^{57}$ Mechanisms for drug interactions and a list of clinically relevant drugs interacting with tacrolimus have been reviewed elsewhere. ${ }^{58}$ 


\section{Corticosteroids}

Several studies revealed that corticosteroids are inducers of Cyp3A. ${ }^{56,59,120,121}$ Accordingly, we and others have described rising tacrolimus levels after steroid withdrawal. ${ }^{59,71,122,123}$ Since the Сур3A enzymes are present in both the intestines and the liver, this may lead to a reduced bioavailability and an increased clearance. Given the large inter-individual differences in Сур3A activity which can differ for 5- to 10 -fold between individuals ${ }^{121}$, it is possible that the influence of steroids on hepatic or intestinal tacrolimus metabolism will vary amongst carriers of different Cyp3A alleles. Since steroids are often prescribed for indications other than graft protection, e.g. gout or COPD exacerbations, the prescribing physician should bear in mind that this may thus decrease tacrolimus concentrations.

\section{Food}

The factors discussed above are more related to the medical care provided by the transplantation centre and the pharmacy. Other factors that can influence variability are directly related to the patient itself. Probably closely related to mechanisms responsible for drug interactions, the intake of food may influence the bio-availability of tacrolimus. The effect of food on the oral bioavailability of tacrolimus when administered as the BID formulation has been investigated before. ${ }^{62,83,124}$ The presence of food decreased both the rate and extent of absorption of tacrolimus, with the effect being most pronounced after a high-fat meal. ${ }^{124}$ It is therefore advised to administer tacrolimus on an empty stomach, i.e. one hour before or two hours after a meal.

However, we have shown that after ingestion of tacrolimus in a fed state vs. fasting state, i.e. together with a continental breakfast, conversion to fed ingestion of tacrolimus, without dose adjustments, did not significantly change tacrolimus trough levels in stable renal transplant recipients. ${ }^{62,125}$ Based on these results, in our centre, the patients are offered the possibility to take Tac BID in the morning simultaneously with breakfast from the second week post-transplant on. ${ }^{62}$ No such reports had been published for the once-daily formulation.

\section{Diarrhoea}

Increased tacrolimus trough concentrations in patients with diarrhoeal illness have been reported in several papers. ${ }^{126-130}$ This does not seem to be the case when diarrhoea is only mild. ${ }^{130}$ A possible explanation for the elevation in tacrolimus levels is increased bioavailability due to increased intestinal permeability through mucosal injury. ${ }^{117}$ Alternatively, as Cyp3A4-enzymes and P-glycoprotein are present in differing amounts throughout the gastrointestinal tract ${ }^{49}$, the bioavailability of tacrolimus may be influenced by changes in gastrointestinal transit time, with shunting of drug to 
areas of lower intestinal metabolism. ${ }^{57}$ Other possible mechanisms for (small) increased tacrolimus levels during diarrhoea concern haemoconcentration, increased absorption as a result of fasted intake of the drug, or reduced hepatic metabolism caused by reduced hepatic blood flow or hepatic dysfunction. ${ }^{116}$

\subsection{Tac QD}

\subsubsection{Development of Tac QD}

Based on the composition of Tac BID capsules, a granule formulation has been developed to prolong the drug release profile of tacrolimus. The objective was to achieve $90 \%$ drug release at 6 to 12 hours. A preparation designated modifiedrelease 4 (MR-4) was found to have appropriate dissolution characteristics and, after administration of the drug in the morning, to yield comparable drug exposure (as measured by the area under the concentration-time curve from 0 to 24 hours postdose; $\mathrm{AUC}_{0-24}$ ) to the immediate release Tac BID formulation. ${ }^{14}$ Due to its different dissolution properties, this preparation is typically released further along the gastrointestinal tract. ${ }^{52}$ With enabling QD dosing, it has been suggested that the primary benefit of the prolonged-release preparation may be improved convenience of medication administration. ${ }^{14,131}$

\subsubsection{Conversion to Tac QD in stable kidney transplant patients}

On the basis of the earlier studies in healthy volunteers kidney transplant patients participating in pharmacokinetic phase II trials were dosed on an 1:1 mg:mg base compared with Tac BID. ${ }^{88,91}$ In stable kidney transplant recipients (at least 6 months after transplantation and stable graft function) Alloway et al. presented an open-label, multi-centre study with a crossover design. ${ }^{88}$ The $90 \%$ confidence intervals for natural logarithmic transformed ratios of the Tac QD to Tac BID at steady state were within the $80-125 \%$ range that defined bioequivalence according to the European Medicines Agency (EMA). ${ }^{101}$ Later, EMA guidelines were altered to specify that in cases of drugs with a narrow therapeutic index, the bioequivalence acceptance interval for were tightened to $90-111 \%{ }^{101}$

The Tac QD formulation was tolerated with a safety profile comparable to that of Tac BID. It has also been shown that the steady state pharmacokinetics of Tac QD was equivalent to Tac BID regardless of gender, race, or presence of diabetes. ${ }^{88}$ Similar results were reported in a 4-period crossover, replicate-design study that compared the pharmacokinetics of once-daily (QD) and twice-daily tacrolimus (BID) in stable renal transplant recipients. ${ }^{91}$ In this study, steady-state tacrolimus exposure and blood trough levels were comparable for both tacrolimus formulations. In addition, both 
formulations were well tolerated, with renal function remaining stable throughout the study.

However, in both studies, as in other clinical conversion studies in stable renal transplant recipients, the average $\mathrm{AUC}_{0-24}$ and the trough level $\left(\mathrm{C}_{\mathrm{min}}\right)$ were consistently 5-15\% lower for Tac QD compared with BID. ${ }^{132-136}$ In the prospective studies, no increases in dose were required in most patients. ${ }^{52}$ In the biggest observational study so far, small increases in the dose requirements were introduced (less than $3 \%$ of relative increase in the entire population sample of 1832 patients) at the time of conversion to a once daily formulation because of modest reductions (of approximately $10 \%$ ) in tacrolimus levels during the first days of administration after conversion. Thereafter, the levels and doses remained stable for the rest of the studied follow up year. ${ }^{137}$ Retrospective analysis of data from a conversion study in 284 adult kidney transplant recipients, however, showed an average decrease in $\mathrm{C}_{\text {min }}$ of $12.7 \pm 24.4 \%$ and a dose increase in more than $50 \%$ of the patients. ${ }^{132}$ Similarly, another retrospective study also showed increased daily tacrolimus dosage after conversion to Tac QD. ${ }^{134}$

An increase in tacrolimus dosage as in some studies cited above might be due to the retrospective nature of the studies, which then reflects an individual clinical decision made by the treating physician. On the other hand, the fact that no dose changes are necessary in most cases might indicate limited clinical relevance of the supposed decrease in drug exposure. However, tacrolimus has a narrow therapeutic index, the extent of reduction in AUC or $C_{\text {min }}$ observed in clinical trials may thus be clinically significant in the lower target range. In this respect, follow-up studies were necessary to confirm the long-term efficacy and safety.

In this respect, patient and graft survival at 2 years after conversion were $100 \%$ and $98.5 \%$, respectively, and the incidence of biopsy proven acute rejection was $6.0 \% .{ }^{138}$ The safety profile was similar to the Tac BID formulation. The incidence of posttransplant diabetes mellitus, hyperlipidaemia, hypertension, infections, renal dysfunction, or hepatic dysfunction was consistent with that observed with Tac BID. ${ }^{138}$ Also, a four-year follow-up study of former phase II trials has delivered evidence that kidney, liver, and heart transplant patients could be maintained for up to four-year post-transplant on modified-release Tac QD dosing with stable renal function, providing similar efficacy and safety to the BID Formulation. ${ }^{139}$

\subsubsection{Tac QD in de novo kidney transplant patients}

In de novo kidney transplant patients, a Phase II study (study FG-506E-12-01) was performed to compare the pharmacokinetics of tacrolimus following once daily administration of Tac QD and twice daily administration of Tac BID. ${ }^{92}$ Comparisons of systemic exposure $\left(A \cup C_{0-24}\right)$, peak concentration $\left(C_{\max }\right)$ and trough levels $\left(C_{24}\right)$ were performed following the first dose and at day 14 and week 6 post-transplantation. The 
study was of 6-week duration. The first total daily dose for Tac QD and Tac BID was comparable; however, the systemic exposure to tacrolimus on Day 1 was approximately 32\% lower for Tac QD than for Tac BID. By Day 14 and Week 6, the geometric mean $\mathrm{AUC}_{0-24}$ ratio of Tac QD:Tac BID (normalised to an equivalent dose for both formulations $\left(0.1 \mathrm{mg} / \mathrm{kg} /\right.$ day)) was 98 and $82 \%$, respectively. ${ }^{92}$ The systemic exposure to tacrolimus for Tac QD was then within $10 \%$ of that for Tac BID, which is comparable to the exposure in the stable conversion studies. Despite the $32 \%$ lower exposure to tacrolimus on Day 1 observed for Tac QD compared with Tac BID, the efficacy and safety of Tac QD was not different to that of Tac BID. In a follow-up study in de novo kidney transplant recipients it has been shown that one-year patient and graft survival were similar for the combination therapy of Tac QD and Mycophenolic Acid (MMF) compared with Tac BID and MMF. ${ }^{140}$ Also, the safety profile of Tac QD was similar to that observed with Tac BID in this study. ${ }^{140}$

Lower drug exposure in the early post-transplant period with Tac QD compared with BID was reported in several other studies, where 20-50\% higher Tac QD doses were required to reach the targeted drug levels. ${ }^{107,141,142}$ The lower systemic exposure to tacrolimus observed for Tac QD on Day 1 has been suggested to be attributed to an absence of the absorption of tacrolimus administered in the evening relative to the morning (as is the case for the Tac BID formulation) on the first day post-transplant. ${ }^{14}$ Notwithstanding, in most cases, use of Tac QD in de novo transplant recipients leads to lower drug exposure at a certain dose compared with Tac BID. The reduction in dose-corrected $\mathrm{AUC}_{0-24}$ appears to be of greater magnitude than that observed in stable transplant patients, and occurs at a time that is potentially the most crucial with regard to achieving adequate immunosuppression. ${ }^{52}$ Careful and frequent monitoring of tacrolimus trough levels is recommended in the first two weeks posttransplant with Tac QD to ensure adequate drug exposure in the immediate posttransplant period. ${ }^{14}$

Another solution for this potential caveat in the very early post-transplant period is to give the first Tac QD dose before engraftment of the kidney. In our centre, this had already been introduced with Tac BID to achieve sufficient immunosuppression without induction therapy early after engraftment. For Tac QD, this concept has been proven to reduce the earlier reported 32\% lower AUC on day 1 to $16 \%$ after initiating tacrolimus therapy before transplant at a dose of $0.1 \mathrm{mg} / \mathrm{kg} / \mathrm{day} .{ }^{143}$ From day 1 on, the regular $0.2 \mathrm{mg} / \mathrm{kg} /$ day were administered resulting in similar exposure between treatment groups. It has been concluded from this study that initiating tacrolimus therapy before transplantation minimizes the exposure differences between Tac QD and Tac BID in the immediate post-transplant period by half and represented an appropriate strategy in treating renal transplant patients. ${ }^{143}$ Similar efficacy and safety results have also been concluded from a multi-centre, placebo-controlled randomized trial where Tac QD has been compared in a double-blind fashion to Tac BID in 
combination with low-dose MMF and corticosteroids without antibody induction, with pre-operative dosing of any tacrolimus formulation. ${ }^{142}$

\subsubsection{Variability of Tac QD}

In de novo kidney transplant recipients treated with Tac QD, between- or inter-subject coefficients of variation (CVs) for $\mathrm{AUC}_{0-24}$ dose were more than $40 \%$ on days 14 and 42 post-transplant. ${ }^{144}$ Similarly, in a study of 41 stable Tac QD-treated kidney transplant recipients, inter-subject steady-state $C V$ s were $47 \%$ and $45 \%$ for dose-adjusted $A \mathrm{CC}_{0-24}$ and $\mathrm{C}_{\min }$, respectively. ${ }^{89}$

A limited number of studies have compared within- or intra-subject pharmacokinetic variability for Tac BID with that of Tac QD. In a conversion study of 41 stable kidney transplant recipients, intrapatient variability of dose-normalized $\mathrm{C}_{\min }$ values was lower for the two formulations $(26.2 \%$ for Tac BID vs. $23.4 \%$ for Tac QD, but not significant). ${ }^{136}$ Alternatively, in a phase II study involving stable liver transplant recipients, intrapatient variability for dose-adjusted tacrolimus exposure was lower for Tac QD than with Tac BID (ratio Advagraf/Prograf=0.594; $p=0.044$ ).$^{90}$ Interestingly, in a study of 67 stable kidney transplant recipients, intrapatient variability in tacrolimus exposure with Tac QD versus Tac BID was 4-fold lower in African-American recipients compared with whites (ratio $Q D / B I D=0.235 ; p=0.02$ )..$^{88}$ The last two studies, however, measured the $\mathrm{AUC}_{0-24}$ for each formulation only on two occasions, which would limit the accuracy for calculation of the intrapatient variability. Formal comparison of within-patient variability for both formulations with $\mathrm{AUC}_{0-24}$ measurement on more frequent occasions had not been described, yet.

In conclusion, both in de novo and in stable kidney transplant recipients Tac QD has been proven to be an effective and safe alternative treatment compared with Tac BID in terms of clinical endpoints such as prevention of acute rejection, patient- and graft survival, with similar side effects. The convenience of once-daily dosing may improve therapy adherence and reduce within-patient variability, which may, eventually, improve long-term graft survival. However, aspects considering its variability and influence of co-ingestion of food had not formerly been investigated yet.

\subsection{Aims and outline of the thesis}

In this thesis, we explored some pharmacokinetic and pharmacogenetic aspects of tacrolimus-based immunosuppressive therapy which may then be implemented in strategies to aim at optimization of tacrolimus use. The aims of this thesis were: 
- To elucidate factors contributing to the pharmacokinetic variability in tacrolimus exposure influenced by pharmacogenetics, steroids, and tacrolimus-food interactions.

- To investigate the pharmacokinetic properties of alternative routes of administration in cases the drug cannot be taken orally, to guarantee graft protection under these conditions.

- To develop reliable TDM strategies to predict drug exposure for Tac QD.

- To assess the intrapatient variability of drug exposure of Tac QD compared with Tac BID.

Specifically, the subsequent chapters elaborate these aspects by answering the following questions:

1. Do genetic polymorphisms of the Cytochrome P450 3A5 (Сyp3A5) and the Pregnane $X$ Receptor (PXR) contribute to the observed increase in dosenormalised tacrolimus trough levels (DnTac) (i.e. the decreased Tarolimus dose requirements during the post-transplant period) after steroid tapering and withdrawal (Chapter 2)?

2. Do alternative routes of tacrolimus administration result in systemic drug exposure? In Chapter 3, the results of a pharmacokinetic study on the relative bioavailability of sublingual and rectal application of tacrolimus compared with the oral capsules in healthy volunteers will be described.

3. Which TDM strategies perform best to predict drug exposure for Tac QD? In Chapter 4 different TDM strategies were developed and validated.

4. What is the influence of a standard continental breakfast on the oral bioavailability of Tac QD (Chapter 5$)$ ?

5. What is the intrapatient variability in 24-hour drug exposure expressed as the 24-hour blood concentration-time curve $\left(\mathrm{AUC}_{0-24}\right)$ of Tac QD compared with Tac BID (Chapter 6)?

The overall conclusions from these studies will be presented during the general discussion in chapter 7 . 


\section{References}

1. Menon V, Sarnak MJ. The epidemiology of chronic kidney disease stages 1 to 4 and cardiovascular disease: a high-risk combination. Am J Kidney Dis 2005;45(1):223-232.

2. Jaarverslag 2017 Nederlandse Transplantatie Stichting (2017). Nederlandse Transplantatie Stichting.

3. Meier-Kriesche HU, Ojo OA, Port FK, et al. Survival improvement among patients with end-stage renal disease: trends over time for transplant recipients and wait-listed patients. J Am Soc Nephrol 2001;12(6):1293-1296.

4. Snoeijs MG, Schaubel DE, Hene R, et al. Kidneys from donors after cardiac death provide survival benefit. J Am Soc Nephrol 2010;21(6):1015-1021.

5. Merrill JP, Murray JE, Harrison JH, et al. Successful homotransplantation of the human kidney between identical twins. J Am Med Assoc 1956;160(4):277-282.

6. Morris PJ. Transplantation--a medical miracle of the 20th century. N Engl J Med 2004;351(26): 2678-2680.

7. van Hooff JP, van Es A, Koolen MI, et al. Less aggressive rejection therapy and low-dose corticosteroids leading to satisfactory cadaveric kidney graft survival and low morbidity rate. Proc Eur Dial Transplant Assoc 1980;17:435-439.

8. Boots JM, van Duijnhoven EM, Christiaans $\mathrm{MH}$, et al. Single-center experience with tacrolimus versus cyclosporine-Neoral in renal transplant recipients. Transpl Int 2001;14(6):370-383.

9. Kahan BD. Cyclosporine. N Engl J Med 1989;321(25):1725-1738.

10. Pritchard DI. Sourcing a chemical succession for cyclosporin from parasites and human pathogens. Drug Discov Today 2005;10(10):688-691.

11. Knoll GA, Bell RC. Tacrolimus versus cyclosporin for immunosuppression in renal transplantation: meta-analysis of randomised trials. BMJ 1999;318(7191):1104-1107.

12. Webster A, Woodroffe RC, Taylor RS, et al. Tacrolimus versus cyclosporin as primary immunosuppression for kidney transplant recipients. Cochrane Database Syst Rev 2005;(4): CD003961.

13. Meier-Kriesche HU, Li S, Gruessner RW, et al. Immunosuppression: evolution in practice and trends, 1994-2004. Am J Transplant 2006;6(5 Pt 2):1111-1131.

14. Advagraf: scientific discussion. European Medicines Agency. 2007 Available at: http://www.ema.europa.eu/

15. Lamb KE, Lodhi S, Meier-Kriesche HU. Long-term renal allograft survival in the United States: a critical reappraisal. Am J Transplant 2011;11(3):450-462.

16. Meier-Kriesche HU, Schold JD, Srinivas TR, et al. Lack of improvement in renal allograft survival despite a marked decrease in acute rejection rates over the most recent era. Am J Transplant 2004;4(3):378-383.

17. Coemans, M., C. Susal, B. Dohler, et al. Analyses of the short- and long-term graft survival after kidney transplantation in Europe between 1986 and 2015. Kidney Int 2018;94(5):964-973.

18. Nankivell BJ, Kuypers DR. Diagnosis and prevention of chronic kidney allograft loss. Lancet 2011; 378(9800):1428-1437.

19. Sellares J, de Freitas DG, Mengel M, et al. Understanding the causes of kidney transplant failure: the dominant role of antibody-mediated rejection and nonadherence. Am J Transplant 2012;12(2): 388-399.

20. Djamali A, Kaufman DB, Ellis TM, et al. Diagnosis and management of antibody-mediated rejection: current status and novel approaches. Am J Transplant 2014;14(2):255-271.

21. Wiebe C, Gibson IW, Blydt-Hansen TD, et al. Evolution and clinical pathologic correlations of de novo donor-specific HLA antibody post kidney transplant. Am J Transplant 2012;12(5):1157-1167.

22. Beland MA, Lapointe I, Noel R, et al. Higher calcineurin inhibitor levels predict better kidney graft survival in patients with de novo donor-specific anti-HLA antibodies: a cohort study. Transpl Int 2017; 30(5):502-509.

23. Opelz G. Tacrolimus trough levels and graft survival. Collaborative Transplant Study Newsletter, 2014. 
24. Borra LC, Roodnat JI, Kal JA, et al. High within-patient variability in the clearance of tacrolimus is a risk factor for poor long-term outcome after kidney transplantation. Nephrol Dial Transplant 2010;25(8):2757-2763.

25. Kahan BD, Welsh M, Schoenberg L, et al. Variable oral absorption of cyclosporine. A biopharmaceutical risk factor for chronic renal allograft rejection. Transplantation 1996;62(5): 599-606.

26. Kapturczak MH, Meier-Kriesche HU, Kaplan B. Pharmacology of calcineurin antagonists. Transplant Proc 2004;36(2 Suppl):25S-32S.

27. Matsuda S, Shibasaki F, Takehana K, et al. Two distinct action mechanisms of immunophilin-ligand complexes for the blockade of T-cell activation. EMBO Rep 2000;1(5):428-434.

28. Jeffrey KL, Camps M, Rommel C, et al. Targeting dual-specificity phosphatases: manipulating MAP kinase signalling and immune responses. Nat Rev Drug Discov 2007;6(5):391-403.

29. Khanna A, Cairns V, Hosenpud JD. Tacrolimus induces increased expression of transforming growth factor-beta1 in mammalian lymphoid as well as nonlymphoid cells. Transplantation 1999;67(4): 614-619.

30. Khanna AK, Hosenpud JD. Cyclosporine induces the expression of the cyclin inhibitor p21. Transplantation 1999;67(9):1262-1268.

31. Gorelik L, Flavell RA. Transforming growth factor-beta in T-cell biology. Nat Rev Immunol 2002;2(1): 46-53.

32. Minguillon J, Morancho B, Kim SJ, et al. Concentrations of cyclosporin A and FK506 that inhibit IL-2 induction in human T cells do not affect TGF-beta1 biosynthesis, whereas higher doses of cyclosporin A trigger apoptosis and release of preformed TGF-beta1. J Leukoc Biol 2005;77(5):748-758.

33. Boyman $\mathrm{O}$, Sprent J. The role of interleukin-2 during homeostasis and activation of the immune system. Nat Rev Immunol 2012;12(3):180-190.

34. Kamel M, Kadian M, Srinivas $T$, et al. Tacrolimus confers lower acute rejection rates and better renal allograft survival compared to cyclosporine. World J Transplant 2016;6(4):697-702.

35. Mayer AD, Dmitrewski J, Squifflet JP, et al. Multicenter randomized trial comparing tacrolimus (FK506) and cyclosporine in the prevention of renal allograft rejection: a report of the European Tacrolimus Multicenter Renal Study Group. Transplantation 1997;64(3):436-443.

36. Pirsch JD, Miller J, Deierhoi $\mathrm{MH}$, et al. A comparison of tacrolimus (FK506) and cyclosporine for immunosuppression after cadaveric renal transplantation. FK506 Kidney Transplant Study Group. Transplantation 1997;63(7):977-983.

37. Almawi WY, Melemedjian OK. Clinical and mechanistic differences between FK506 (tacrolimus) and cyclosporin A. Nephrol Dial Transplant 2000;15(12):1916-1918.

38. Plosker GL, Foster RH. Tacrolimus: a further update of its pharmacology and therapeutic use in the management of organ transplantation. Drugs 2000;59(2):323-389.

39. van Hooff JP, Christiaans MH. Use of tacrolimus in renal transplantation. Transplant Proc 1999;31(8): 3298-3299.

40. van Hooff JP, Gelens M, Boots JM, et al. Preservation of renal function and cardiovascular risk factors. Transplant Proc 2006;38(7):1987-1991.

41. Mori A, Suko M, Kaminuma O, et al. IL-2-induced IL-5 synthesis, but not proliferation, of human CD4+ T cells is suppressed by FK506. J Immunol 1997;158(8):3659-3665.

42. Miller JL, Ericson SG. Cyclosporin a and tacrolimus (FK506) differentially alter T-cell receptor expression in vivo. Immunopharmacol Immunotoxicol 2007;29(1):105-118.

43. van de Plas A, Dackus J, Christiaans $\mathrm{MH}$, et al. A pilot study on sublingual administration of tacrolimus. Transpl Int 2009;22(3):358-359.

44. Pea F. Pharmacokinetics in Everyday Clinical Practice. Torino 2012.

45. Knops N, Levtchenko E, van den Heuvel B, et al. From gut to kidney: transporting and metabolizing calcineurin-inhibitors in solid organ transplantation. Int J Pharm 2013;452(1-2):14-35.

46. Zhang Y, Benet LZ. The gut as a barrier to drug absorption: combined role of cytochrome P450 $3 \mathrm{~A}$ and P-glycoprotein. Clin Pharmacokinet 2001;40(3):159-168.

47. Wacher VJ, Silverman JA, Zhang $Y$, et al. Role of P-glycoprotein and cytochrome P450 $3 A$ in limiting oral absorption of peptides and peptidomimetics. J Pharm Sci 1998;87(11):1322-1330. 
48. Paine MF, Hart HL, Ludington SS, et al. The human intestinal cytochrome P450 "pie". Drug Metab Dispos 2006;34(5):880-886.

49. Thorn M, Finnstrom N, Lundgren S, et al. Cytochromes P450 and MDR1 mRNA expression along the human gastrointestinal tract. Br J Clin Pharmacol 2005;60(1):54-60.

50. Fojo AT, Ueda K, Slamon DJ, et al. Expression of a multidrug-resistance gene in human tumors and tissues. Proc Natl Acad Sci U S A 1987;84(1):265-269.

51. Venkataramanan R, Swaminathan A, Prasad T, et al. Clinical pharmacokinetics of tacrolimus. Clin Pharmacokinet 1995;29(6):404-430.

52. Barraclough KA, Isbel NM, Johnson DW, et al. Once- versus twice-daily tacrolimus: are the formulations truly equivalent? Drugs 2011;71(12):1561-1577.

53. Collin C, Boussaud V, Lefeuvre S, et al. Sublingual tacrolimus as an alternative to intravenous route in patients with thoracic transplant: a retrospective study. Transplant Proc 2010;42(10):4331-4337.

54. Nicolai S, Bunyavanich S. Hypersensitivity reaction to intravenous but not oral tacrolimus. Transplantation 2012;94(9):e61-63.

55. van Dieren JM, van Bodegraven AA, Kuipers EJ, et al. Local application of tacrolimus in distal colitis: feasible and safe. Inflamm Bowel Dis 2009;15(2):193-198.

56. Christians $U$, Jacobsen W, Benet LZ, et al. Mechanisms of clinically relevant drug interactions associated with tacrolimus. Clin Pharmacokinet 2002;41(11):813-851.

57. Staatz CE, Tett SE. Clinical pharmacokinetics and pharmacodynamics of tacrolimus in solid organ transplantation. Clin Pharmacokinet 2004;43(10):623-653.

58. van Gelder T. Drug interactions with tacrolimus. Drug Saf 25(10): 707-712.

59. Shimada T, Terada A, Yokogawa K, et al. Lowered blood concentration of tacrolimus and its recovery with changes in expression of СурЗ $\mathrm{A}$ and $\mathrm{P}$-glycoprotein after high-dose steroid therapy. Transplantation 2002;74(10):1419-1424.

60. Tuteja S, Alloway RR, Johnson JA, et al. The effect of gut metabolism on tacrolimus bioavailability in renal transplant recipients. Transplantation 2001;71(9):1303-1307.

61. Advagraf: Summary of Product Characateristics. (2007, last updated 04/15/2019). European Medicines Agency.

62. Christiaans M, van Duijnhoven E, Beysens T, et al. Effect of breakfast on the oral bioavailability of tacrolimus and changes in pharmacokinetics at different times posttransplant in renal transplant recipients. Transplant Proc 30(4): 1271-1273.

63. Wallemacq $\mathrm{P}$, Armstrong VW, Brunet $\mathrm{M}$, et al. Opportunities to optimize tacrolimus therapy in solid organ transplantation: report of the European consensus conference. Ther Drug Monit 31(2): 139-152.

64. Ree van LMB, D.D. Algemene Farmacologie, Elsevier. 2003.

65. Tamargo J, Le Heuzey JY, Mabo P. Narrow therapeutic index drugs: a clinical pharmacological consideration to flecainide. Eur J Clin Pharmacol 2015;71(5):549-567.

66. Andrews LM, Li Y, De Winter BCM, et al. Pharmacokinetic considerations related to therapeutic drug monitoring of tacrolimus in kidney transplant patients. Expert Opin Drug Metab Toxicol 2017;13(12):1225-1236.

67. de Jonge $H$, Naesens $M$, Kuypers DR. New insights into the pharmacokinetics and pharmacodynamics of the calcineurin inhibitors and mycophenolic acid: possible consequences for therapeutic drug monitoring in solid organ transplantation. Ther Drug Monit 2009;31(4):416-435.

68. Ekberg H, Tedesco-Silva H, Demirbas A, et al. Reduced exposure to calcineurin inhibitors in renal transplantation. N Engl J Med 2007;357(25):2562-2575.

69. van Gelder T. Within-patient variability in immunosuppressive drug exposure as a predictor for poor outcome after transplantation. Kidney Int 2014;85(6):1267-1268.

70. Budde K, Glander P. Pharmacokinetic principles of immunosuppressive drugs. Ann Transplant 2008;13(3):5-10.

71. Kuypers DR, Claes K, Evenepoel P, et al. Time-related clinical determinants of long-term tacrolimus pharmacokinetics in combination therapy with mycophenolic acid and corticosteroids: a prospective study in one hundred de novo renal transplant recipients. Clin Pharmacokinet 2004;43(11): 741-762. 
72. Undre NA, Schafer A. Factors affecting the pharmacokinetics of tacrolimus in the first year after renal transplantation. European Tacrolimus Multicentre Renal Study Group. Transplant Proc 1998;30(4): 1261-1263.

73. Israni AK, Riad SM, Leduc R, et al. Tacrolimus trough levels after month 3 as a predictor of acute rejection following kidney transplantation: a lesson learned from DeKAF Genomics. Transpl Int 2013;26(10):982-989.

74. Ekberg H, Mamelok RD, Pearson TC, et al. The challenge of achieving target drug concentrations in clinical trials: experience from the Symphony study. Transplantation 2009;87(9):1360-1366.

75. Laskow DA, Vincenti F, Neylan J, et al. Phase II FK 506 multicenter concentration control study: oneyear follow-up. Transplant Proc 1995;27(1):809-811.

76. Undre NA, van Hooff J, Christiaans M, et al. Low systemic exposure to tacrolimus correlates with acute rejection. Transplant Proc 1999;31(1-2):296-298.

77. Heemann U, Abramowicz D, Spasovski G, et al. Endorsement of the Kidney Disease Improving Global Outcomes (KDIGO) guidelines on kidney transplantation: a European Renal Best Practice (ERBP) position statement. Nephrol Dial Transplant 2011;26(7):2099-2106.

78. Kasiske BL, Zeier MG, Chapman JR, et al. KDIGO clinical practice guideline for the care of kidney transplant recipients: a summary. Kidney Int 2010;77(4):299-311.

79. Spencer CM, Goa KL, GillisJC. Tacrolimus. An update of its pharmacology and clinical efficacy in the management of organ transplantation. Drugs 1997;54(6):925-975.

80. Braun F, Schutz E, Peters B, et al. Pharmacokinetics of tacrolimus primary immunosuppression in kidney transplant recipients. Transplant Proc 2001;33(3):2127-2128.

81. Macchi-Andanson M, Charpiat B, Jelliffe RW, et al. Failure of traditional trough levels to predict tacrolimus concentrations. Ther Drug Monit 2001;23(2):129-133.

82. Pisitkun T, Eiam-Ong S, Chusil S, et al. The roles of $\mathrm{C} 4$ and AUCO-4 in monitoring of tacrolimus in stable kidney transplant patients. Transplant Proc 2002;34(8):3173-3175.

83. Stolk LM, van Duijnhoven EM, Christiaans $\mathrm{MH}$, et al. Trough levels of tacrolimus. Ther Drug Monit 2002;24(4): 573; author reply 573-574.

84. Wong KM, Shek CC, Chau KF, et al. Abbreviated tacrolimus area-under-the-curve monitoring for renal transplant recipients. Am J Kidney Dis 2000;35(4):660-666.

85. Braun F, Peters B, Schutz E, et al. Therapeutic drug monitoring of tacrolimus early after liver transplantation. Transplant Proc 2002;34(5):1538-1539.

86. Armendariz Y, Pou L, Cantarell C, et al. Evaluation of a limited sampling strategy to estimate area under the curve of tacrolimus in adult renal transplant patients. Ther Drug Monit 2005;27(4):431-434.

87. Scholten EM, Cremers SC, Schoemaker RC, et al.. AUC-guided dosing of tacrolimus prevents progressive systemic overexposure in renal transplant recipients. Kidney Int 2005;67(6):2440-2447.

88. Alloway R, Steinberg S, Khalil K, et al. Conversion of stable kidney transplant recipients from a twice daily Prograf-based regimen to a once daily modified release tacrolimus-based regimen. Transplant Proc 2005;37(2):867-870.

89. Benkali K, Rostaing L, Premaud A, et al. Population pharmacokinetics and Bayesian estimation of tacrolimus exposure in renal transplant recipients on a new once-daily formulation. Clin Pharmacokinet 2010;49(10):683-692.

90. Florman S, Alloway R, Kalayoglu M, et al. Conversion of stable liver transplant recipients from a twicedaily Prograf-based regimen to a once-daily modified release tacrolimus-based regimen. Transplant Proc 2005;37(2):1211-1213.

91. van Hooff J, van der Walt I, Kallmeyer K, et al. Pharmacokinetics in stable kidney transplant recipients after conversion from twice-daily to once-daily tacrolimus formulations. Ther Drug Monit 2012;34(1): 46-52.

92. Wlodarczyk Z, Squifflet JP, Ostrowski M, et al. Pharmacokinetics for once- versus twice-daily tacrolimus formulations in de novo kidney transplantation: a randomized, open-label trial. Am J Transplant 2009;9(11):2505-2513.

93. Op den Buijsch RA, Christiaans MH, Stolk LM, et al. Tacrolimus pharmacokinetics and pharmacogenetics: influence of adenosine triphosphate-binding cassette $B 1(A B C B 1)$ and cytochrome (CYP) 3A polymorphisms. Fundam Clin Pharmacol 2007;21(4):427-435. 
94. Marquet P, Bedu A, Monchaud C, et al. Pharmacokinetic Therapeutic Drug Monitoring of Advagraf in More Than 500 Adult Renal Transplant Patients, Using an Expert System Online. Ther Drug Monit 2018;40(3):285-291.

95. Hoogtanders K, van der Heijden J, Christiaans M, et al. Therapeutic drug monitoring of tacrolimus with the dried blood spot method. J Pharm Biomed Anal 2007;44(3):658-664.

96. Cheung CY, van der Heijden J, Hoogtanders K, et al. Dried blood spot measurement: application in tacrolimus monitoring using limited sampling strategy and abbreviated AUC estimation. Transpl Int 2008;21(2):140-145.

97. Hoogtanders K, van der Heijden J, Christiaans M, et al. Dried blood spot measurement of tacrolimus is promising for patient monitoring. Transplantation 2007;83(2):237-238.

98. Denhaerynck K, Dobbels F, Cleemput I, et al. Prevalence, consequences, and determinants of nonadherence in adult renal transplant patients: a literature review. Transpl Int 2005;18(10): 1121-1133.

99. Butler JA, Roderick P, Mullee $M$, et al. Frequency and impact of nonadherence to immunosuppressants after renal transplantation: a systematic review. Transplantation 2004;77(5): 769-776.

100. Shuker N, van Gelder T, Hesselink DA. Intra-patient variability in tacrolimus exposure: causes, consequences for clinical management. Transplant Rev (Orlando) 2015;29(2):78-84.

101. Note for Guidance on the Investigation of Bioavailability and Bioequivalence (NFG on BA BE). E. M. Agency. London. 2010.

102. van Duijnhoven E, Christiaans $M$, Schafer A, et al. Tacrolimus dosing requirements in diabetic and nondiabetic patients calculated from pretransplantation data. Transplant Proc 1998;30(4):1266-1267.

103. Cheung $C Y$, Op den Buijsch RA, Wong KM, et al. Influence of different allelic variants of the Cyp3A and $A B C B 1$ genes on the tacrolimus pharmacokinetic profile of Chinese renal transplant recipients. Pharmacogenomics 2006;7(4):563-574.

104. de Jonge $H$, Kuypers DR. Pharmacogenetics in solid organ transplantation: current status and future directions. Transplant Rev (Orlando) 2008;22(1):6-20.

105. Macphee IA, Fredericks S, Tai T, et al. Tacrolimus pharmacogenetics: polymorphisms associated with expression of cytochrome p4503A5 and P-glycoprotein correlate with dose requirement. Transplantation 2002;74(11):1486-1489.

106. Haufroid V, Wallemacq $P$, Van Kerckhove V, et al. Cyp3A5 and ABCB1 polymorphisms and tacrolimus pharmacokinetics in renal transplant candidates: guidelines from an experimental study. Am J Transplant 2006;6(11):2706-2713.

107. Crespo M, Mir M, Marin M, et al. De novo kidney transplant recipients need higher doses of Advagraf compared with Prograf to get therapeutic levels. Transplant Proc 2009;41(6):2115-2117.

108. Andrews PA, Sen M, Chang RW. Racial variation in dosage requirements of tacrolimus. Lancet 1996; 348(9039):1446.

109. Haufroid V, Mourad M, Van Kerckhove V, et al. The effect of Cyp3A5 and MDR1 (ABCB1) polymorphisms on cyclosporine and tacrolimus dose requirements and trough blood levels in stable renal transplant patients. Pharmacogenetics 2004;14(3):147-154.

110. Press RR, Ploeger BA, den Hartigh J, et al. Explaining variability in tacrolimus pharmacokinetics to optimize early exposure in adult kidney transplant recipients. Ther Drug Monit 2009;31(2):187-197.

111. Forman BM. Polymorphisms in promiscuous PXR: an explanation for interindividual differences in drug clearance? Pharmacogenetics 2001;11(7):551-552.

112. Hesselink DA, Bouamar R, Elens $L$, et al. The role of pharmacogenetics in the disposition of and response to tacrolimus in solid organ transplantation. Clin Pharmacokinet 2014;53(2):123-139.

113. Tang JT, Andrews LM, van Gelder T, et al. Pharmacogenetic aspects of the use of tacrolimus in renal transplantation: recent developments and ethnic considerations. Expert Opin Drug Metab Toxicol 2016;12(5):555-565.

114. van Gelder T, van Schaik RH, Hesselink DA. Pharmacogenetics and immunosuppressive drugs in solid organ transplantation. Nat Rev Nephrol 2014;10(12):725-731. 
115. Yu Y, Teerenstra S, Neef $C$, et al. A comparison of the intrasubject variation in drug exposure between generic and brand-name drugs: a retrospective analysis of replicate design trials. $\mathrm{Br} \mathrm{J}$ Clin Pharmacol 2016;81(4):667-678.

116. Mittal N, Thompson JF, Kato $\mathrm{T}$, et al. Tacrolimus and diarrhea: pathogenesis of altered metabolism. Pediatr Transplant 2001;5(2):75-79.

117. Watkins PB. The barrier function of Cyp3A4 and P-glycoprotein in the small bowel. Adv Drug Deliv Rev 1997;27(2-3):161-170.

118. Christians U, Schmidt G, Bader A, et al. Identification of drugs inhibiting the in vitro metabolism of tacrolimus by human liver microsomes. Br J Clin Pharmacol 1996;41(3):187-190.

119. Prasad TN, Stiff DD, Subbotina N, et al. FK 506 (Tacrolimus) metabolism by rat liver microsomes and its inhibition by other drugs. Res Commun Chem Pathol Pharmacol 1994;84(1):35-46.

120. El-Sankary W, Plant NJ, Gibson GG, et al. Regulation of the Cyp3A4 gene by hydrocortisone and xenobiotics: role of the glucocorticoid and pregnane X receptors. Drug Metab Dispos 2000;28(5): 493-496.

121. McCune JS, Hawke RL, LeCluyse EL, et al. In vivo and in vitro induction of human cytochrome P4503A4 by dexamethasone. Clin Pharmacol Ther 2000;68(4):356-366.

122. Anglicheau $\mathrm{D}$, Flamant $\mathrm{M}$, Schlageter $\mathrm{MH}$, et al. Pharmacokinetic interaction between corticosteroids and tacrolimus after renal transplantation. Nephrol Dial Transplant 2003;18(11):2409-2414.

123. van Duijnhoven EM, Boots JM, Christiaans $\mathrm{MH}$, et al. Increase in tacrolimus trough levels after steroid withdrawal. Transpl Int 2003;16(10):721-725.

124. Bekersky I, Dressler D, Mekki Q. Effect of time of meal consumption on bioavailability of a single oral 5 mg tacrolimus dose. J Clin Pharmacol 2001;41(3):289-297.

125. van Duijnhoven EM, Christiaans MH, Boots JM, et al. Evidence that fasting does not significantly affect trough levels of tacrolimus in stable renal transplant recipients. Transplant Proc 2002;34(5): 1723-1725.

126. Zylber-Katz E, Granot E. Abrupt increase of tacrolimus blood levels during an episode of Shigella infection in a child after liver transplantation. Ther Drug Monit 2001;23(6):647-649.

127. Fruhwirth $\mathrm{M}$, Fischer $\mathrm{H}$, Simma B, et al. Elevated tacrolimus trough levels in association with mycophenolate mofetil-induced diarrhea: a case report. Pediatr Transplant 2001;5(2):132-134.

128. Matsui A, Arakawa Y, Momoya T, et al.. Apparently increased trough levels of tacrolimus caused by acute infantile diarrhea in two infants with biliary atresia after liver transplantation. Acta Paediatr Jpn 1996;38(6):699-701.

129. Fruhwirth $M$, Fischer $H$, Simma B, et al. Rotavirus infection as cause of tacrolimus elevation in solidorgan-transplanted children. Pediatr Transplant 2001;5(2):88-92.

130. van Boekel GA, Aarnoutse RE, van der Heijden JJ, et al. Effect of mild diarrhea on tacrolimus exposure. Transplantation 2012;94(7):763-767.

131. First MR, Fitzsimmons WE. Modified release tacrolimus. Yonsei Med J 2004;45(6):1127-1131.

132. de Jonge $H$, Kuypers $D R$, Verbeke $K$, et al. Reduced $C 0$ concentrations and increased dose requirements in renal allograft recipients converted to the novel once-daily tacrolimus formulation. Transplantation 2010;90(5):523-529.

133. Diez Ojea B, Alonso Alvarez M, Aguado Fernandez S, et al. Three-month experience with tacrolimus once-daily regimen in stable renal allografts. Transplant Proc 2009;41(6):2323-2325.

134. Hougardy JM, Broeders N, Kianda M, et al. Conversion from Prograf to Advagraf among kidney transplant recipients results in sustained decrease in tacrolimus exposure. Transplantation 2011; 91(5):566-569.

135. Iaria G, Sforza D, Angelico R, et al. Switch from twice-daily tacrolimus (Prograf) to once-daily prolonged-release tacrolimus (Advagraf) in kidney transplantation. Transplant Proc 2011;43(4): 1028-1029.

136. Wehland $M$, Bauer S, Brakemeier S, et al. Differential impact of the Cyp3A5* 1 and Cyp3A5*3 alleles on pre-dose concentrations of two tacrolimus formulations. Pharmacogenet Genomics 2011;21(4): 179-184. 
137. Guirado L, Cantarell C, Franco A, et al. Efficacy and safety of conversion from twice-daily to once-daily tacrolimus in a large cohort of stable kidney transplant recipients. Am J Transplant 2011;11(9): 1965-1971.

138. Alloway R, Steinberg S, Khalil K, et al. Two years postconversion from a prograf-based regimen to a once-daily tacrolimus extended-release formulation in stable kidney transplant recipients. Transplantation 2007;83(12):1648-1651.

139. van Hooff JP, Alloway RR, Trunecka P, et al. Four-year experience with tacrolimus once-daily prolonged release in patients from phase II conversion and de novo kidney, liver, and heart studies. Clin Transplant 2011;25(1):E1-12.

140. Silva HT Jr, Yang HC, Abouljoud M, et al. One-year results with extended-release tacrolimus/MMF, tacrolimus/MMF and cyclosporine/MMF in de novo kidney transplant recipients. Am J Transplant 2007;7(3):595-608.

141. Andres A, Delgado-Arranz M, Morales E, et al. Extended-release tacrolimus therapy in de novo kidney transplant recipients: single-center experience. Transplant Proc 2010;42(8):3034-3037.

142. Kramer BK, Charpentier B, Backman L, et al. Tacrolimus once daily (ADVAGRAF) versus twice daily (PROGRAF) in de novo renal transplantation: a randomized phase III study. Am J Transplant 2010; 10(12):2632-2643.

143. Wlodarczyk Z, Ostrowski M, Mourad M, et al. Tacrolimus pharmacokinetics of once- versus twicedaily formulations in de novo kidney transplantation: a substudy of a randomized phase III trial. Ther Drug Monit 20102;34(2):143-147.

144. Saint-Marcoux F, Debord J, Undre N, et al. Pharmacokinetic modeling and development of Bayesian estimators in kidney transplant patients receiving the tacrolimus once-daily formulation. Ther Drug Monit 2010;32(2):129-135. 


\section{Chapter 2}

Increase in tacrolimus exposure after steroid tapering is influenced by Cyp3A5 and Pregnane X Receptor genetic polymorphisms in renal transplant recipients

F. Stifft, S.M.J. v. Kuijk, O.Bekers, M.H.L. Christiaans

Nephrology, dialysis, transplantation. 2018;33 (9):1668-1675 


\section{Abstract}

\section{Background}

Tacrolimus, a drug for prevention of rejection after kidney transplantation, has a narrow therapeutic window and is metabolised by the Cytochrome P540 3a (Cyp3A) system. Tacrolimus exposure increases after steroid tapering in many patients. The Pregnane $X$ Receptor (PXR) -a mediator for Cyp3A- has a steroid receptor and might regulate Cyp3A5 activity depending on Single Nuclear Polymorphisms (SNPs) of Cyp3A5 or PXR. This may contribute to differences in tacrolimus exposure after steroid tapering.

\section{Methods}

In a cohort of renal transplant recipients, the influence of Cyp3A5 and PXR SNPS (A7635G, C8055T and C25385T) on the Dose-normalized tacrolimus trough concentrations $\left(\mathrm{DnC}_{0}\right)$ and their potential interaction with each other after steroid taper was analyzed by linear regression. Eligible were all 83 outpatient renal transplant patients on tacrolimus and steroids in a pharmacokinetic steady state at least six weeks after transplantation and of whom blood was available for genetic analysis.

\section{Results}

Compared with the Cyp3A5 $*_{1} / *_{3}$ genotype, the Cyp3A5*3/*3 SNP showed a significantly stronger increase in $\mathrm{DnC}_{0}$ after steroid taper $(+0.29 \mu \mathrm{g} / \mathrm{l} / \mathrm{mg} ; \mathrm{p}=0.002)$. Of the tested PXR SNPS, PXR G7635G individuals had a significant stronger increase in $\mathrm{DnC}_{0}$ (compared to $A 7635 A+0.31 \mu \mathrm{g} / \mathrm{l} / \mathrm{mg} ; \mathrm{p}=0.02$ ), with a weaker increase in $A 7635 G$ heterozygotes $(+0.17 \mu \mathrm{g} / \mathrm{l} / \mathrm{mg} ; \mathrm{p}=0.124)$. There was neither interaction nor association between Cyp3A5 and PXR SNPs.

\section{Conclusions}

The magnitude of $\mathrm{DnC}_{0}$ rise due to steroid taper after renal transplantation is related to Cyp3A5 SNPs. Independently, the PXR G7635G SNP is related to this rise, proving the role of PXR in tacrolimus metabolism. 


\section{Introduction}

Tacrolimus is a frequently used immunosuppressive after renal transplantation. Because of its narrow therapeutic window between underexposure (with risk of rejection) and overexposure (with risk of side-effects) therapeutic drug monitoring is advised. In clinical practice, dosing is adapted on the trough concentration (as surrogate for the exposure). With increasing time post-transplant, a decrease in the dosage of tacrolimus required to maintain similar trough concentrations has been reported. ${ }^{1}$ A decrease in tacrolimus clearance with time has been considered to be the reason for this. ${ }^{2}$

However, tacrolimus dose requirements also diminish after steroid withdrawal. ${ }^{1,3-5}$ Clinically relevant increases ( $>20 \%$ rise of trough concentration) have been reported in $43 \%$ of the patients after withdrawal of $5 \mathrm{mg}$ and in $61 \%$ of patients after withdrawal of $10 \mathrm{mg}$ prednisolone. ${ }^{4}$ This rise in trough concentration after steroid tapering has been demonstrated also to be present when tapering takes place more than 6 months after transplantation, when mechanisms like improving graft function, liver function and rise in haemoglobin or albumin concentrations are less likely to occur. ${ }^{1,4}$

The reason for this phenomenon has not been clearly elucidated yet. Relevant pharmacokinetic interaction between corticosteroids and tacrolimus has been linked to Cytochrome P450 3a (Cyp3A) and P-glycoprotein (P-gp), a drug efflux pump produced by the multidrug-resistance-1 (MDR-1) gene. ${ }^{3}$ Since steroids are reported to induce the activity of Cyp3A enzymes ${ }^{6}$, it has been postulated that steroid tapering may result in diminished Сyp3A5 activity. ${ }^{4}$ Сyp3A enzymes in the liver and small intestine are major enzymes responsible for tacrolimus metabolism leading to a substantial first-pass effect. ${ }^{7,8}$ Tacrolimus exposure is also related to single nucleotide polymorphisms (SNPS) of Cyp3A5. To our knowledge, it is not known whether the effect of tapering steroids on tacrolimus exposure is different between these SNPs.

The induction of $С y p 3 A$, on the other hand, is mediated by the Pregnane $\mathrm{X}$ receptor (PXR, also called NR1/2: nuclear receptor $1 / 2) .{ }^{9}$ It is a regulatory factor of the Nuclear Receptor family with a steroid receptor and is involved in the upregulation of many drug-metabolizing enzymes and drug transporters. ${ }^{10,11}$ The induction of the Cyp3A gene in response to treatment with a variety of compounds, such as the glucocorticoid dexamethasone, the antibiotic rifampicin, and the antimycotic clotrimazole $^{9,12}$, could then be linked to the PXR.

Several SNPS in the $P X R$-gene have been found to increase Cyp3A induction. ${ }^{6}$ However, it is unknown whether the systemic exposure to tacrolimus is then (in-) directly related to the inducing capacity mediated by SNPs in the PXR-gene. If so, carriers of these inducing SNPs should have higher tacrolimus requirements while on steroids and thus have a more pronounced increase in dose-corrected trough concentrations after steroid withdrawal compared to the wild type allele-carriers. 
In this study we hypothesize that the steroid-mediated induction of the PXR upregulates the metabolic clearance through Cyp3A5 and, therefore, contributes to the rise in tacrolimus exposure after steroid tapering or withdrawal. The aim of the current study is then to determine whether the observed increase in tacrolimus exposure after steroid tapering is associated with certain SNPs of the Cyp3A5 or the $P X R$ gene and whether there is an interaction between SNPs of the Cyp3A5 and the $P X R$ gene. To test this hypothesis, we retrospectively analyzed data in a population of renal transplant patients of whom we obtained relevant biochemical, pharmaceutical and genetic data.

\section{Methods}

\section{Patients and samples and clinical data}

Between December 2002 and February 2005, leftover ethylenediaminetetraacetic acid (EDTA) blood samples of renal transplant patients routinely visiting our outpatient clinic were collected. Patient data and tacrolimus trough concentrations were retrieved from the patient files and hospital information system. Collection, storage, and use of blood and patient data have been performed in agreement with FEDERA (Federation of Dutch University Medical Centres) Code of Conduct (www.nfu.nl). Secondary usage of this leftover material was approved by the Medical Ethical Committee of the Maastricht University Medical Centre (MEC 04-188).

All clinically stable renal transplant patients at least 6 weeks posttransplant on tacrolimus-based immunosuppression and whose leftover samples for DNA analysis were available were included. In addition, pharmacokinetic steady state tacrolimus trough concentrations had to be present at a prednisolone dose of $10 \mathrm{mg} / \mathrm{day}$ and/or $5 \mathrm{mg} /$ day and after complete steroid withdrawal. According to our centre protocol at that time, prednisolone dose was $10 \mathrm{mg}$ during the first six weeks, $7.5 \mathrm{mg}$ and $5 \mathrm{mg}$ for four weeks during the ensuing two months, respectively, and complete withdrawal thereafter, provided there were no signs of rejection or a higher degree of immunization.

Tacrolimus trough concentration in pharmacokinetic steady state was defined as at least 7 days after last prednisolone dose reduction and/or last tacrolimus dose change. Excluded from analysis were patients who were treated for acute rejection within one month before measurements and who were suffering from any condition influencing absorption or elimination of tacrolimus, like gastrointestinal disorders, hepatic dysfunction, or medication interfering with tacrolimus pharmacokinetics. 


\section{Parameters}

We collected the following biochemical, pharmaceutical and genetic parameters from hospital clinical and laboratory files: 'Age', 'Time since transplantation', 'Body weight', 'Dose-normalized trough concentration $\left(D_{n} C_{0}\right)$ ' (calculated from 'Tacrolimus trough level [ $\left.C_{0}\right]$ )' and 'Tacrolimus total daily dose') , 'Haemoglobin' and 'serum albumin'. The Cyp3A5*1 and *3 alleles as well as three different SNPs of the PXR gene (A7635G, C8055T and C25385T) were determined. The choice of the PXR SNPs was based on previous reports: $A 7635 \mathrm{G}$ and $C 8055 \mathrm{~T}$ are associated with a higher magnitude of (intestinal) Cyp3A inducibility ${ }^{6}$, while $C 25385 T$ has been reported to be related to differences in tacrolimus apparent clearance. ${ }^{13}$

\section{DNA-analysis}

Genomic DNA from a venous blood sample was extracted according to the manufacturers' instructions (Qiagen, Leusden, the Netherlands). Real-time polymerase chain reaction (PCR) fluorescence resonance transfer (FRET) assays were used for genotyping with the LightCycler (Roche Diagnostics, Almere, The Netherlands) as described previously. ${ }^{14}$

\section{Statistical analysis}

Baseline characteristics of the patients were presented as mean and standard deviation (SD), or absolute value and percentage for all three prednisolone dosages. $\mathrm{DnC}_{0}$ values were compared using an independent samples t-test. The unadjusted association of both Cyp3A5 and PXR SNPs and the $\mathrm{DnC}_{0}$ value for $10 \mathrm{mg}$ steroid use were first computed using linear regression. In addition, we computed the adjusted associations after correction for all other SNPs and potential confounding factors (i.e. gender, age, weight at $10 \mathrm{mg}$ steroid use (baseline), elapsed time since transplantation, haemoglobin concentration, serum albumin concentration, $\mathrm{DnC}_{0}$ at $10 \mathrm{mg}$ steroid use, time interval between 10 and $0 \mathrm{mg}$ steroid use). These potential confounders were tested for significance using backward stepwise elimination. To assess interactions between Cyp3A5 and PXR SNPs for the individual slopes of the $\mathrm{DnC}_{0}$ value depending on steroid use, we computed interaction terms and tested using linear regression.

Next, we determined $\mathrm{DnC}_{0}$ changes over the three measures by performing a linear regression analysis per patient to obtain each patients individual $\mathrm{DnC}_{0}$ slope. These slopes were used for subsequent analyses using the same methods as for the associations with $\mathrm{DnC}_{0}$ at $10 \mathrm{mg}$ steroid use. Finally, we used Fisher's Exact test to assess whether there were any associations between Cyp3A5- and PXR-SNPs. All analyses were performed using SPSS, version 23 (IBM, Armonk, NY, USA). P-values $\leq 0.05$ were considered statistically significant. 


\section{Results}

Patients screened for this study received a renal transplant between March 1993 and January 2003. After this time our centre protocol changed into early steroid withdrawal. Therefore, patients transplanted after 2003 could not be included. By collecting leftover EDTA blood samples for DNA analyses between December 2002 and February 2005 we were able to collect material for all patients that were under regular control in our centre. This way, we had a database consisting of 325 patients. When retrospectively applying the criteria for eligibility (at least 6 weeks posttransplant on tacrolimus-based immunosuppression, pharmacokinetic steady state tacrolimus trough concentration at prednisolone dose of $10 \mathrm{mg} /$ day and/or $5 \mathrm{mg} / \mathrm{day}$ and after complete steroid withdrawal) 105 patients were eligible for further analyses. After application of the exclusion criteria, we had tacrolimus trough concentration measurements in 83 patients after complete steroid withdrawal. Of these, 66 patients also had a measurement at $5 \mathrm{mg}$ prednisolone dose and 81 at $10 \mathrm{mg}$ prednisolone dose. Tacrolimus trough concentrations were available at all 3 prednisolone doses in 64 patients. Of these, 17 patients did not have a stable tacrolimus trough concentration at prednisolone $5 \mathrm{mg}$ and 2 patients at $10 \mathrm{mg}$. Baseline characteristics of the entire cohort for the respective prednisolone dosages are shown in Table 2.1.

\section{Correlation of Cyp3A5 and PXR SNPs for $\mathrm{DnC}_{0}$ at $10 \mathrm{mg}$ steroid dose}

The unadjusted (univariable) and adjusted (multivariable) associations between the tested Cyp3A5 and PXR SNPs and $\mathrm{DnC}_{0}$ for $10 \mathrm{mg}$ steroid dose (baseline measurement) are shown in Table 2.2. In the multivariable model, all SNPs were entered simultaneously together with all potential relevant confounders (i.e. except $\mathrm{DnC}_{0}$ at $10 \mathrm{mg}$ steroid use and time interval between 10 and $0 \mathrm{mg}$ steroid use). None of the potential confounders were statistically significant and they were therefore left out of the final multivariable model. Both in the univariable and multivariable analysis, Cyp3A5 carrier state was the only significant factor correlated with $\mathrm{DnC}_{0}: *^{*} / *^{*} \mathrm{SNP}$ individuals had a $42 \%$ higher $\mathrm{DnC}_{0}$ in comparison to the ${ }^{*} 1 / *^{*} \mathrm{SNP}$ individuals $(1.4 \mu \mathrm{g} / \mathrm{l} / \mathrm{mg}$ vs. $0.8 \mu \mathrm{g} / \mathrm{l} / \mathrm{mg})$. At baseline, i.e. under $10 \mathrm{mg}$ prednisolone dose, none of the PXR SNPs showed a statistically significant difference in $\mathrm{DnC}_{0}$. 
Table 2.1 Baseline characteristics of the study cohort at three different prednisolone dosages ${ }^{\mathrm{a}}$.

\begin{tabular}{|c|c|c|c|}
\hline & $\begin{array}{c}\text { Prednisolone } 10 \\
(\mathrm{~N}=81)\end{array}$ & $\begin{array}{c}\text { Prednisolone } 5 \\
(\mathrm{~N}=66)\end{array}$ & $\begin{array}{c}\text { Prednisolone } 0 \\
(\mathrm{~N}=83)\end{array}$ \\
\hline Age (years) & $49.1(12.5)$ & $48.4(11.9)$ & $50.2(12.7)$ \\
\hline Gender (male) & $50(61.0 \%)$ & 39 (59\%) & $51(61.4 \%)$ \\
\hline Time since transplant (days) & $88.5(276.8)$ & 151 (218.9) & $580(759.5)$ \\
\hline Days on stable prednidolone dose & $41.5(132.8)$ & $48.8(115.1)$ & 95.1 (293.9) \\
\hline Kreatinin $(\mu \mathrm{mol} / \mathrm{l})$ & $222.5(168.6)$ & $168.9(67.7)$ & $146.1(50.8)$ \\
\hline $\mathrm{Hb}(\mathrm{mmol} / \mathrm{l})$ & $6.8(5.6)$ & $7.4(1.2)$ & $8.1(1.1)$ \\
\hline Albumin (g/l) & $33.8(5.1)$ & $39.5(4.3)$ & $38.8(4.1)$ \\
\hline ALAT (mmol/l) & $28.1(20.4)$ & $21.3(9.8)$ & $24.7(14.1)$ \\
\hline Daily Tacrolimus dose (mg) & 15.5 (9) & $10.2(6.1)$ & $6.7(4.4)$ \\
\hline Tacrolimus trough concentration $(\mu \mathrm{g} / \mathrm{l})$ & $13.9(4.1)$ & $11.9(3.7)$ & $9.6(3.2)$ \\
\hline Dn trough concentration $(\mu \mathrm{g} / \mathrm{l} / \mathrm{mg})^{\mathrm{b}}$ & $1.2(0.7)$ & $1.6(1.1)$ & $1.9(1.1)$ \\
\hline \multicolumn{4}{|l|}{ Cyp3A5 } \\
\hline$* 3 / * 3$ & $52(64.2 \%)$ & $43(65.1 \%)$ & $54(65 \%)$ \\
\hline$* 1 / * 3$ & $29(35.8 \%)$ & $23(34.9 \%)$ & 29 (34.9\%) \\
\hline \multicolumn{4}{|l|}{ PXR C25385T } \\
\hline $\mathrm{CC}$ & 38 (46.9\%) & $36(54.5 \%)$ & 39 (47.0\%) \\
\hline $\mathrm{CT}$ & 35 (43.2\%) & 25 (37.9\%) & 35 (43.4\%) \\
\hline TT & $8(9.8 \%)$ & $5(7.6 \%)$ & $8(9.6 \%)$ \\
\hline \multicolumn{4}{|l|}{ PXR A7635G } \\
\hline$A A$ & $33(40.7 \%)$ & $24(36.4 \%)$ & 33 (39.7\%) \\
\hline$A G$ & $29(35.8 \%)$ & 25 (37.9\%) & 30 (36.1\%) \\
\hline GG & $19(23.5 \%)$ & $16(25.7 \%)$ & 19 (24.0\%) \\
\hline \multicolumn{4}{|l|}{ PXR C8055T } \\
\hline $\mathrm{CC}$ & 56 (69.1\%) & 48 (72.3\%) & 59 (71.1\%) \\
\hline CT & $22(27.2 \%)$ & $15(22.7 \%)$ & 22 (26.5\%) \\
\hline $\mathrm{TT}$ & $3(3.7 \%)$ & 3 (4.5\%) & $3(3.6 \%)$ \\
\hline
\end{tabular}

${ }^{\mathrm{a}}$ Data are presented as mean (standard deviation) or absolute value (\%); ${ }^{\mathrm{b}} \mathrm{Dn}=$ Dose-normalized.

\section{Influence of steroid withdrawal on $\mathrm{DnC}_{0}$}

$\mathrm{DnC}_{0}$ increased by $58 \%$ (from a mean of 1.2 to $1.9 \mu \mathrm{g} / \mathrm{l} / \mathrm{mg}$ ) after withdrawal of prednisolone from $10 \mathrm{mg}$ to $0 \mathrm{mg}$ daily $(\mathrm{p}<0.001$, Table 2.1). As illustrated in Figure 2.1, $\mathrm{DnC}_{0}$ increased with every step of prednisolone tapering with a large interindividual variability. The individual changes in $\mathrm{DnC}_{0}$ after steroid withdrawal are demonstrated in Figure 2.2. By considering (arbitrarily) a $20 \%$ change to be clinically relevant, about $75 \%$ of the individuals $(n=62)$, had a clinically relevant increase up to $260 \%$ (with a single outlier of $>400 \%$ ), while 18 remained stable and three individuals had a clinically relevant decline in $\mathrm{DnC}_{0}$. Since we observed one patient with an extreme increase in $\mathrm{DnC}_{0}$ after steroid withdrawal of more than $400 \%$ (Figure 2.2), we performed a sensitivity analysis in which the extreme outlier was omitted from the analyses. The sensitivity analysis did not result in different conclusions, nor did it result in different effect sizes or the signs of effects (results not shown), as described in the following sections. 
Table 2.2 Association of Pregnane X Receptor- and cytochrome gene single nucleotide polymorphisms with dose-normalized tacrolimus concentration for $10 \mathrm{mg}$ of steroid use.

\begin{tabular}{lcccccc}
\hline & Univariable & \multicolumn{5}{c}{ Multivariable } \\
\cline { 2 - 7 } & Coefficient & SE & p-value & Coefficient & SE & p-value \\
\hline PXR C25385T (CC is reference) & & & & & & \\
CT & -0.02 & 0.15 & 0.916 & -0.10 & 0.14 & 0.497 \\
TT & -0.11 & 0.25 & 0.650 & -0.27 & 0.24 & 0.257 \\
PXR A7635G (AA is reference) & & & & & & \\
AG & -0.05 & 0.15 & 0.740 & 0.13 & 0.17 & 0.446 \\
GG & 0.24 & 0.17 & 0.170 & 0.15 & 0.21 & 0.477 \\
PXR C8055T (CC is reference) & & & & & & \\
CT & 0.01 & 0.17 & 0.977 & 0.04 & 0.18 & 0.832 \\
TT & 0.56 & 0.38 & 0.151 & 0.58 & 0.38 & 0.143 \\
Cyp3A5 $(* 3 / * 3$ is reference) & & & & & & \\
$\quad * 1 / * 3$ & -0.66 & 0.13 & $<0.001$ & -0.71 & 0.14 & $<0.001$ \\
\hline
\end{tabular}

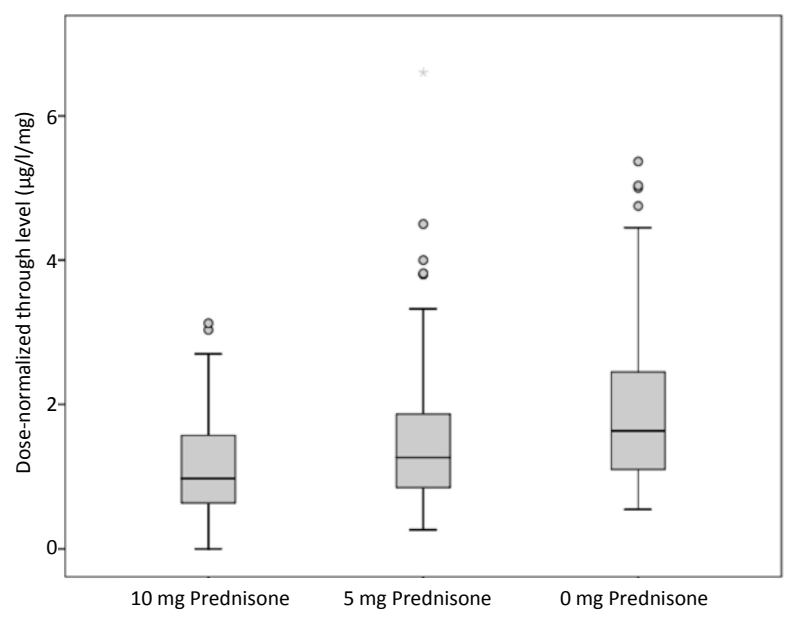

Figure 2.1 Boxplots ${ }^{a}$ of the dose-normalized Tacrolimus Trough concentrations for three different Prednisolone dosages. ${ }^{\text {a }}$ The band inside the box shows the median value, whereas the bottom and top of the box represent the first and third quartiles, respectively. The small vertical lines at the end of the whiskers denote the lowest and highest value that are still within 1.5 times the interquartile range (IQR). The individual points are the outliers that fall outside of this boundary 


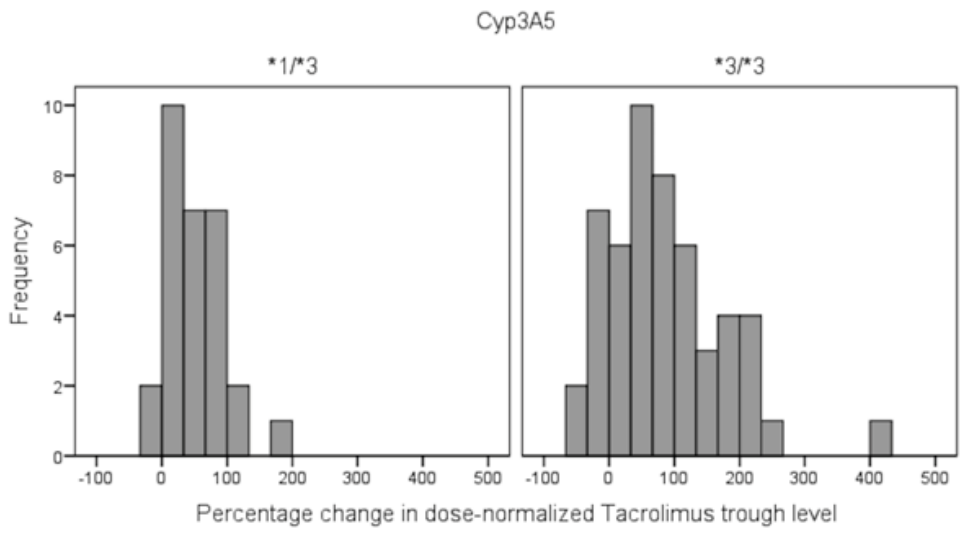

Figure 2.2 Distribution of frequencies of percentage change in $\mathrm{DnC}_{0}$ after prednisolone dose reduction from $10 \mathrm{mg} /$ day to $0 \mathrm{mg} /$ day.

Relationship of Cyp3A5 and PXR SNPs with the change in $\mathrm{DnC}_{0}$ for different steroid dosages

In accordance with literature, Сyp $3 \mathrm{~A}^{*} 3 /{ }^{*} 3$ homozygotes have a higher $\mathrm{DnC}_{0}$ compared to Cyp $3 A 5^{*} 1 /{ }^{*} 3$ carriers. This finding is valid at all prednisolone dosages (Figure 2.3 and Table 2.3). As shown in Table 2.3, not only the $\mathrm{DnC}_{0}$ but also its increase by steroid tapering is significantly higher in Cyp3A5*3/*3 individuals (+64\%) compared with Cyp3A5*1/*3 individuals $(+37 \%)(p=<0.001)$.

Table 2.3 Main outcomes stratified by Cyp3A5 genotype ${ }^{a}$

\begin{tabular}{lcccccc}
\hline & \multicolumn{5}{c}{ Cyp3A5*3/*3 } & \multicolumn{5}{c}{ Cyp3A5 *1/*3 } \\
\cline { 2 - 7 } & Pred 10 & Pred 5 & Pred 0 & Pred 10 & Pred 5 & Pred 0 \\
& $(\mathrm{N}=52)$ & $(\mathrm{N}=43)$ & $(\mathrm{N}=54)$ & $(\mathrm{N}=29)$ & $(\mathrm{N}=23)$ & $(\mathrm{N}=29)$ \\
\hline Daily Tacrolimus dose $(\mathrm{mg})$ & $11.5(6.1)$ & $7.3(3.8)$ & $4.6(2.2)$ & $23.0(9.3)$ & $15.5(6.0)$ & $10.8(5.2)$ \\
Tacrolimus trough concentration $(\mu \mathrm{g} / \mathrm{l})$ & $13.1(3.5)$ & $11.8(3.9)$ & $9.2(3.0)$ & $15.3(4.7)$ & $12.0(3.2)$ & $10.3(3.3)$ \\
Dn trough concentration $(\mu \mathrm{g} / \mathrm{l} / \mathrm{mg})^{\mathrm{b}}$ & $1.4(0.7)$ & $2.0(1.2)$ & $2.3(1.1)$ & $0.8(0.3)$ & $0.9(0.4)$ & $1.1(0.5)$ \\
\hline
\end{tabular}

${ }^{\mathrm{a}}$ Data are presented as mean (standard deviation) or absolute value (\%); ${ }^{\mathrm{b}} \mathrm{Dn}=$ Dose-normalized.

The univariable and multivariable associations between Cyp3A5 and PXR SNPs and the change in $\mathrm{DnC}_{0}$ with every $5 \mathrm{mg}$ decline in prednisolone dose are depicted in Table 2.4. In the multivariable model, all SNPs were entered together with all potential confounders. As none of the latter were statistically significant related to the outcome parameter, the final multivariable model only contains the Cyp3A5 and PXR SNPs. For Сур3A5, both the univariable and the multivariable model show that, compared with 
Cyp3A5*1/*3 heterozygotes, Cyp3A5*3/*3 homozygotes had a clinically significant additional $\pm 0.30 \mu \mathrm{g} / \mathrm{l} / \mathrm{mg}$ increase in $\mathrm{DnC}_{0}$ for every $5 \mathrm{mg}$ steroid taper $(\mathrm{p}=0.002)$.

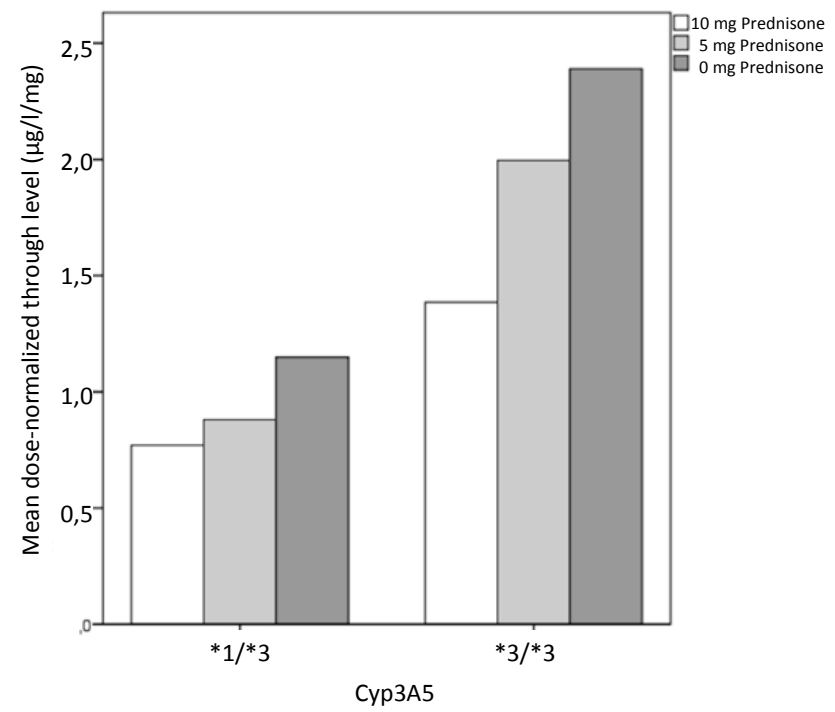

Figure 2.3 Mean DnC0 values stratified by the Сyp3A5 genotype.

Table 2.4 Association of Pregnane $\mathrm{X}$ Receptor- and cytochrome gene single nucleotide polymorphisms with steroid-decrease induced dose-normalized tacrolimus concentration increase.

\begin{tabular}{lcccccc}
\hline & Univariable & \multicolumn{3}{c}{ Multivariable } \\
\cline { 2 - 7 } & Coefficient & SE & P-value & Coefficient & SE & P-value \\
\hline PXR C25385T (CC is reference) & & & & & & \\
CT & 0.07 & 0.09 & 0.489 & 0.04 & 0.09 & 0.644 \\
TT & -0.16 & 0.16 & 0.308 & -0.11 & 0.15 & 0.486 \\
PXR A7635G (AA is reference) & & & & & & \\
AG & -0.02 & 0.10 & 0.837 & 0.17 & 0.11 & 0.138 \\
GG & 0.11 & 0.11 & 0.316 & 0.31 & 0.13 & 0.020 \\
PXR C8055T (CC is reference) & & & & & & \\
CT & -0.08 & 0.11 & 0.478 & -0.18 & 0.11 & 0.110 \\
TT & -0.50 & 0.24 & 0.044 & -0.72 & 0.25 & 0.006 \\
Cyp3A5 (*3/*3 is reference) & & & & & & \\
$* 1 / * 3$ & -0.30 & 0.09 & 0.001 & -0.29 & 0.09 & 0.002 \\
\hline
\end{tabular}

In comparison to the homozygote PXR A7635A genotype, the A7635G heterozygotes had a trend towards a higher increase in $D_{n C}(+0.17 \mu \mathrm{g} / \mathrm{l} / \mathrm{mg}$ for every $5 \mathrm{mg}$ prednisolone tapering), while for the $67635 G$ homozygotes this increase was statistically and clinically significant and nearly twice as high $(+0.31 \mu \mathrm{g} / \mathrm{l} / \mathrm{mg} ; \mathrm{p}=0.02$, 
Figure 2.4). In contrast, the homozygote PXR T8055T genotype had a statistically and clinically relevant lower $\mathrm{DnC}_{0}$ compared with the homozygote PXR C8055C genotype in both univariable and multivariable analyses $(-0,50$ and $-0,72$ resp. for every $5 \mathrm{mg}$ prednisolon tapering). However, interpretation of this has to be cautious because of the small number of individuals with PXR T8055T ( $n=3$, Table 2.1) and the fact that there was no indication for a dose-effect relationship (no decline in the C8055T genotype).

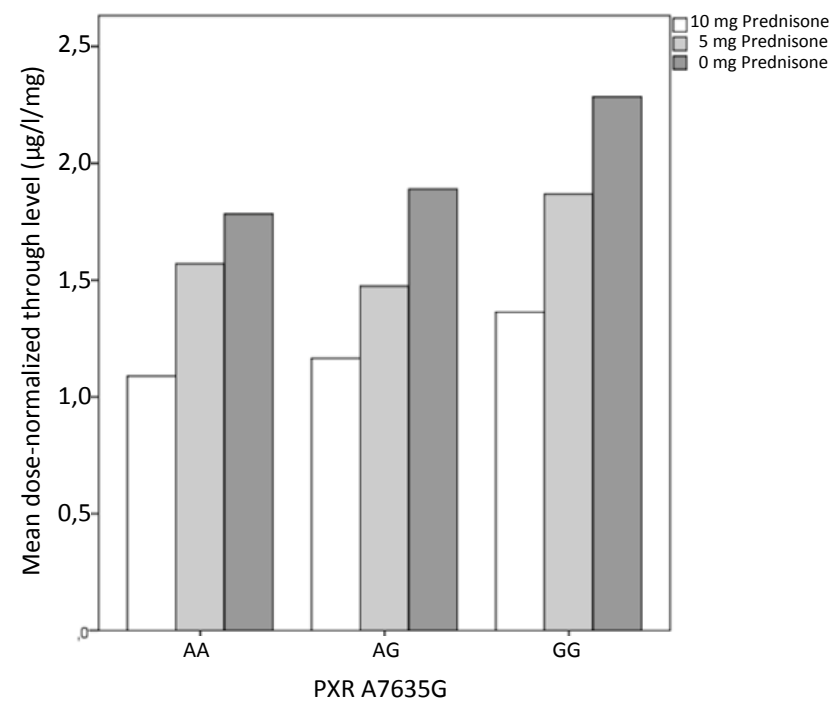

Figure 2.4 Mean $\mathrm{DnC}_{0}$ values stratified by the PXR 7635 genotype at different prednisolone doses/day.

There were no statistically significant interactions between Cyp3A5 and the two statistically significant PXR SNPs (interaction with PXRA7635G GG: B=0.14, SE $=0.23$, $\mathrm{p}=0.559$; interaction with PXRC8055T TT: $\mathrm{B}=-0.70, \mathrm{SE}=0.48, \mathrm{p}=0.149$ ). Fishers Exact test revealed no associations between the presence of PXR SNPs and Cyp3A5 SNPs. $P$-values derived from these tests were $0.840,0.131$, and 0.433 , respectively, for $P X R$ C25385T, PXR A7635G, and PXR C8055T.

\section{Discussion}

This study was designed to determine whether the observed increase in tacrolimus exposure after steroid tapering correlates with certain SNPs of the Cyp3A5 or the PXR gene and whether these SNPs showed any interaction. First, we confirmed the former 
finding of rising $\mathrm{DnC}_{0}$ with steroid tapering (Figure 2.1) with a magnitude consistent with an earlier report from our group. ${ }^{4}$ Second, in line with the Cyp3A5 activity, carriers of the Cyp3A5 *1 genotype had a lower $\mathrm{DnC}_{0}$ compared to the CYP $3 A 5 * 3$ SNP homozygotes (Figure 2.3 and Tables 2.2 and 2.3). Third, not only do Cyp3A5 *1-carriers have a lower $\mathrm{DnC}_{0}$, but a new finding was that they also exhibit a significant approximately $30 \%$ lower rise after steroid taper compared to the Cyp3A5 *3/*3 SNP. Finally, we identified a correlation between the PXR 7635 carrier state on the change in $\mathrm{DnC}_{0}$ and that this correlation is independent from the Cyp3A5 carrier state.

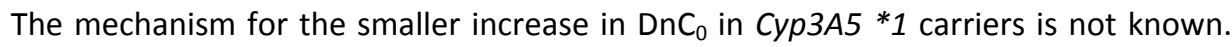
Whereas the increased drug clearance in stable long-term post-transplant patients in Cyp3A5*1 carriers has extensively been described and reviewed ${ }^{15-18}$, their diminished increase in $\mathrm{DnC}_{0}$ over time has only been described by Kuypers et al. who found a $39 \%$ increase in dose-corrected tacrolimus exposure only in the Cyp3A5*3/*3 group during the first 5 years after renal transplant. ${ }^{19}$ The majority of this increase was already within first year post transplant. As most of the steroid reduction takes place during this first year and we found a $25 \%$ increase in drug exposure $/ 5 \mathrm{mg}$ steroid reduction, we conclude that this increase in tacrolimus exposure can be primarily attributed to the steroid taper. This is strengthened by the fact that the time since transplantation of the steroid tapering was not related to the change in tacrolimus exposure in our analysis.

More recently, it has been suggested that the phenomenon of maturation of tacrolimus exposure in the first year after renal transplantation observed in Cyp3A5 *3/*3 homozygous patients can partly be explained by a (steroid tapering-related) decline in Cyp3A4 activity (measured by diminished apparent oral clearance of midazolam) and a progressive increase in haematocrit. ${ }^{20}$ We measured a progressive increase of the haemoglobin concentration and assume that this is equivalent to an increase in haematocrit. However, we did not establish a significant relation of the rise in haemoglobin concentration and the increasing dose corrected tacrolimus concentration during the total time of observation, which was longer than the reported 1-year period in the study of de Jonge et al.. ${ }^{20}$

Since the PXR is a transcriptional regulator of Cyp3A $5^{21-23}$ and this regulation is mediated by steroids ${ }^{24,25}$, carriers of inducing SNPs could then have higher tacrolimus requirements while on steroids and thus have a more pronounced increase in $\mathrm{DnC}_{0}$ after steroid withdrawal compared with the wild-type allele carriers. Our analysis revealed two PXR SNPs to be related to the change in the $\mathrm{DnC}_{0}$ with steroid tapering (Table 2.4). First, compared to PXR A7635A patients, patients with the homozygote G7635G allele had an $0.31 \mu \mathrm{g} / \mathrm{l} / \mathrm{mg}$ higher increase in $\mathrm{DnC}_{0}$ after complete steroid withdrawal and this was nearly double the increase of heterozygote PXR A7635G carriers $(p=0.02)$. These results are in line with the results of Zhang et al, who found a two-fold higher Cyp3A4 mRNA content after 2 days rifampicine exposure in 
homozygous $G 7635 G$ carriers compared to homozygous $A 7635 A$ carriers. ${ }^{26}$ This should translate into a higher Сур3A activity. Reducing the inducing influence of steroids through dose reduction would then result in diminished Cyp3A activity leading to a bigger rise in $\mathrm{DnC}_{0}$. Notably, at a stable steroid dose we did not find differences in DnC $_{0}$ between heterozygote PXR A7635G carriers and homozygote PXR G7635G carriers. However, PXR G7635G homozygotes display a significantly stronger increase after steroid withdrawal which is compatible with our hypothesis that carriers of inducing SNPs have higher tacrolimus requirements while on steroids and thus have a more pronounced increase in $\mathrm{DnC}_{0}$ after steroid withdrawal. Our analysis revealed that this stronger increase in $\mathrm{DnC}_{0}$ is independent of the Cyp3A5 genotype. One has to be aware that the PXR 7635 genotype was the only tested genotype not in Hardy Weinberg (HW) equilibrium: Our study cohort had a relative abundance of homozygous $7635 \mathrm{G}$ patients. We were unable to identify any plausible explanation for this finding in this population that was not preselected by any other parameter than the availability of steady state tacrolimus trough concentration while on prednisolone (10 $\mathrm{mg}$ and/or $5 \mathrm{mg}$ ) and after complete tapering of the prednisolone. Also, the entire pharmacogenetic renal transplant database $(n=325$; without differences in patient characteristics compared to the studied cohort) was in a Hardy Weinberg equilibrium. We therefore think that the HW disequilibrium of the $A 7635 G$ genotype is due to coincidence.

The second SNP statistically related to the change in the $\mathrm{DnC}_{0}$ with steroid tapering was the PXR T8055T genotype. It showed a significantly lower $\mathrm{DnC}_{0}$ increase compared to the heterozygote PXR C8055T or the PXR C8055C reference (multivariable coefficient $-0.72, p=0.06$, Table 2.4 ), which means there is less effect of steroid withdrawal. This is not consistent with what would be expected given the intestinal Cyp3A inducibility as shown by Zhang et al.. ${ }^{6}$ One should consider that, in our study, only three patients carried this PXR T8055T genotype. So no firm conclusions can be drawn from these findings concerning the C8055T SNP, unless they are confirmed in an independent larger cohort.

An advantage of our study was that we had longitudinal data of pharmacokinetically stable patients at different phases of steroid taper during the first 2 years of transplantation instead of studying cross sectional data. Since we adapted our immunosuppressive protocol towards early steroid withdrawal in our centre later on, we are unable to extend the number of patients in this study. As could expected in a white population of this size, we lack in this study Cyp3A5 ${ }^{*} 1 / *^{*} 1$ homozygotes and therefore we have to refrain to make any conclusion about them. Given the low genotype frequency of the PXR T8055T SNP we could not elucidate in detail the potential influence of this apparently rare SNP.

In conclusion, we confirmed the clinically relevant increase in tacrolimus exposure due to steroid tapering in renal transplantation. This phenomenon is not time dependent and probably largely explains the reported increase in drug exposure in the first year 
post-transplant. Given the large inter-patient variability, the fact that tacrolimus exposure increases in all Cyp3A5 groups after steroid tapering and the dose-response relation with every $5 \mathrm{mg}$ prednisolone taper, our advice is to monitor tacrolimus trough concentration after every steroid dose adaptation. Above all, we found that the rise in $\mathrm{DnC}_{0}$ after steroid tapering will be larger in Cyp3A5*3 homozygotes compared to Cyp3A5*1 carriers (and probably Cyp3A5 $\left.{ }^{*} 1\right|^{*} 1$ homozygotes). In addition, we also demonstrated that some SNPS of PXR gene (especially G7635G but also possibly $T 8055 T$ ) were related to a clinically relevant change in tacrolimus exposure due to steroid tapering and that this was independent of the Cyp3A5 SNP. Thereby, this study is the first clinical study showing that the steroid receptor of $P X R$ might be of clinical relevance for tacrolimus metabolism. 


\section{References}

1. Kuypers DR, Claes K, Evenepoel P, et al. Time-related clinical determinants of long-term tacrolimus pharmacokinetics in combination therapy with mycophenolic acid and corticosteroids: a prospective study in one hundred de novo renal transplant recipients. Clin Pharmacokinet 2004;43(11):741-762.

2. Staatz CE, Tett SE. Clinical pharmacokinetics and pharmacodynamics of tacrolimus in solid organ transplantation. Clin Pharmacokinet 2004;43(10):623-653.

3. Anglicheau D, Flamant $\mathrm{M}$, Schlageter $\mathrm{MH}$, et al. Pharmacokinetic interaction between corticosteroids and tacrolimus after renal transplantation. Nephrol Dial Transplant 2003;18(11):2409-414.

4. van Duijnhoven EM, Boots JM, Christiaans $\mathrm{MH}$, et al. Increase in tacrolimus trough levels after steroid withdrawal. Transpl Int 2003;16(10):721-725.

5. Hesselink DA, Ngyuen $\mathrm{H}$, Wabbijn $\mathrm{M}$, et al. Tacrolimus dose requirement in renal transplant recipients is significantly higher when used in combination with corticosteroids. $\mathrm{Br} \mathrm{J}$ Clin Pharmacol 2003;56(3):327-330.

6. Zhang J, Kuehl P, Green ED, et al. The human pregnane $X$ receptor: genomic structure and identification and functional characterization of natural allelic variants. Pharmacogenetics 2001;11(7):555-572.

7. Shimada T, Terada A, Yokogawa K, et al. Lowered blood concentration of tacrolimus and its recovery with changes in expression of Сур3A and P-glycoprotein after high-dose steroid therapy. Transplantation 2002;74(10):1419-1424.

8. Tuteja S, Alloway RR, Johnson JA, et al. The effect of gut metabolism on tacrolimus bioavailability in renal transplant recipients. Transplantation 2001;71(9):1303-1307.

9. Burk O, Koch I, Raucy J, et al. The induction of cytochrome P450 3A5 (Cyp3A5) in the human liver and intestine is mediated by the xenobiotic sensors pregnane $X$ receptor (PXR) and constitutively activated receptor (CAR). J Biol Chem 2004;279(37):38379-38385.

10. Kliewer SA, Moore JT, Wade L, et al. An orphan nuclear receptor activated by pregnanes defines a novel steroid signaling pathway. Cell 1998;92(1):73-82.

11. Ma X, Idle JR, Gonzalez FJ. The pregnane X receptor: from bench to bedside. Expert Opin Drug Metab Toxicol 2008;4(7):895-908.

12. Lehmann JM, McKee DD, Watson MA, et al. The human orphan nuclear receptor PXR is activated by compounds that regulate Cyp3A4 gene expression and cause drug interactions. J Clin Invest 1998;102(5):1016-1023.

13. Benkali K, Premaud A, Picard N, et al. Tacrolimus population pharmacokinetic-pharmacogenetic analysis and Bayesian estimation in renal transplant recipients. Clin Pharmacokinet 2009;48(12): 805-816.

14. Op Den Buijsch R. Pharmacokinetics and Pharmacogenetics of Tacrolimus in Renal Transplant Patients Pharmacokinetics. Maastricht University Clinical Chemistry, 2007.

15. Hesselink DA, van Schaik RH, van der Heiden IP, et al. Genetic polymorphisms of the Cyp3A4, Cyp3A5, and MDR-1 genes and pharmacokinetics of the calcineurin inhibitors cyclosporine and tacrolimus. Clin Pharmacol Ther 2003;74(3):245-254.

16. Tada H, Tsuchiya N, Satoh S, et al. Impact of Cyp3A5 and MDR1(ABCB1) C3435T polymorphisms on the pharmacokinetics of tacrolimus in renal transplant recipients. Transplant Proc 2005;37(4): 1730-1732.

17. de Jonge $H$, Kuypers DR. Pharmacogenetics in solid organ transplantation: current status and future directions. Transplant Rev (Orlando) 2008;22(1):6-20.

18. Thervet $E$, Anglicheau $D$, Legendre $C$, et al. Role of pharmacogenetics of immunosuppressive drugs in organ transplantation. Ther Drug Monit 2008;30(2):143-150.

19. Kuypers DR, de Jonge $H$, Naesens $M$, et al. Cyp3A5 and Cyp3A4 but not MDR1 single-nucleotide polymorphisms determine long-term tacrolimus disposition and drug-related nephrotoxicity in renal recipients. Clin Pharmacol Ther 2007;82(6):711-725. 
20. de Jonge $\mathrm{H}$, Vanhove $\mathrm{T}$, de Loor $\mathrm{H}$, et al. Progressive decline in tacrolimus clearance after renal transplantation is partially explained by decreasing Cyp3A4 activity and increasing haematocrit. $\mathrm{Br} \mathrm{J}$ Clin Pharmacol 2015;80(3):548-559.

21. Lamba JK, Lin YS, Schuetz EG, et al. Genetic contribution to variable human Cyp3A-mediated metabolism. Adv Drug Deliv Rev 2002;54(10):1271-1294.

22. Wang H, LeCluyse EL. Role of orphan nuclear receptors in the regulation of drug-metabolising enzymes. Clin Pharmacokinet 2003;42(15):1331-1357.

23. Zhou SF. Structure, function and regulation of P-glycoprotein and its clinical relevance in drug disposition. Xenobiotica 2008;38(7-8):802-832.

24. Pascussi JM, Drocourt L, Fabre JM, et al. Dexamethasone induces pregnane $X$ receptor and retinoid $X$ receptor-alpha expression in human hepatocytes: synergistic increase of Cyp3A4 induction by pregnane $X$ receptor activators. Mol Pharmacol 2000;58(2):361-372.

25. Pascussi JM, Drocourt L, Gerbal-Chaloin S, et al. Dual effect of dexamethasone on Cyp3A4 gene expression in human hepatocytes. Sequential role of glucocorticoid receptor and pregnane $X$ receptor. Eur J Biochem 2001;268(24):6346-6358.

26. Zhang $\mathrm{Y}$, Benet LZ. The gut as a barrier to drug absorption: combined role of cytochrome $\mathrm{P} 4503 \mathrm{~A}$ and P-glycoprotein. Clin Pharmacokinet 2001;40(3):159-168. 


\title{
Chapter 3
}

Rectal and sublingual administration of tacrolimus: a single dose pharmacokinetic study in healthy volunteers

\author{
F. Stifft, F. Vanmolkot, I. Scheffers, L. van Bortel, C. Neef, M. Christiaans
}

British Journal of Clinical Pharmacology 2014;78(5):996-1004 


\section{Abstract}

\section{Aims}

The immunosuppressant tacrolimus is usually administered orally. When this is not feasible, other routes of administration may be useful. Previous research suggested that tacrolimus may be applied sublingually or rectally. Pharmacokinetic data are sparse. The aim of this study was to investigate and compare the pharmacokinetics of these alternative formulations with the orally administered tacrolimus.

\section{Methods}

Three single, fixed dose formulations of tacrolimus were administered in a random sequence in 18 healthy subjects, using a cross-over study design. For sublingual administration, $3 \mathrm{mg}$ of powder obtained from oral capsules was applied under the tongue for a period of 15 minutes without swallowing, with mouth rinsing afterwards. For rectal administration, a suppository containing $15 \mathrm{mg}$ of the oral powder was used. Oral administration consisted of $7 \mathrm{mg}$ of instant release tacrolimus capsules (Prograf). Main pharmacokinetic outcome parameters were compared by ANOVA.

\section{Results}

Sublingual administration showed no clinically significant exposure, contrary to rectal administration, where all subjects had clinically relevant exposure, with a lower relative bioavailability (70\%), a lower $C_{\max }$, and a later $\mathrm{T}_{\max }$ compared with oral administration.

\section{Conclusion}

Sublingual administration of single dose tacrolimus does not result in systemic exposure if care is taken not to swallow saliva and to rinse the oral cavity afterwards. Rectal administration of tacrolimus results in clinically relevant systemic exposure and might represent an alternative formulation in case oral administration is not feasible. When used as a topical agent, systemic side effects should be considered. 


\section{Introduction}

Tacrolimus is a commonly used immunosuppressant in the field of organ transplantation. It is available as a capsule for oral administration. In some clinical conditions (e.g. nausea, vomiting, sedation, or intubation) oral administration is less feasible. An intravenous (iv) formulation is available, however, iv administration has been associated with an increased incidence of adverse drug reactions compared with oral administration. ${ }^{1,2}$ Several reasons for this increased toxicity have been proposed. First, an increased risk of overexposure owing to unfortunate dose calculations, excessive oral to iv conversion rates or inappropriate dilutions for infusion. ${ }^{3}$ Second, hypersensitivity to polyoxyethylated hydrogenated castor oil, a constituent of the intravenous solution. ${ }^{4}$ Besides, iv administration cannot be used for maintenance therapy. In this context an alternative route of administration would be useful.

Previously, Reams et al. measured therapeutic trough concentrations after sublingual tacrolimus administration $(0.04 \mathrm{mg} / \mathrm{kg})$ in lung transplantation recipients suffering from cystic fibrosis. ${ }^{5}$ Likewise, a recently published paper reports significant tacrolimus exposure in six liver transplant recipients. ${ }^{6}$ In a pilot study of our group with three renal transplant patients we could not confirm tacrolimus absorption after sublingual application. ${ }^{7}$ Likewise, Romero et al. report a case of sublingual tacrolimus administration in a renal transplant patient in which the blood concentration-time profile observed suggests absorption in the lower digestive tract rather than the sublingual mucosa. ${ }^{8}$

Systemic exposure to tacrolimus has been demonstrated in healthy rats treated with high doses of rectally administered tacrolimus. ${ }^{9}$ Previous studies in patients with distal colitis suggest rectally administered tacrolimus is clinically effective and well tolerated. ${ }^{10,11}$ We previously observed significant blood tacrolimus concentrations after rectal administration of tacrolimus in renal transplant patients. ${ }^{12}$

Only few data on the pharmacokinetics and safety of sublingual and rectal tacrolimus administration in humans are available. ${ }^{5-11}$ We therefore designed a pharmacokinetic study to investigate sublingual and rectal administration of tacrolimus and to compare it with orally administered tacrolimus in healthy volunteers.

\section{Methods}

\section{Design}

This study was an open label, single dose, three period crossover study (Table 3.1). The administration sequence was randomized and balanced. Randomization was done by drawing a random envelope containing the number of the stratum. The study was approved by the local medical ethics committee and was in accordance with the 
ethical standards of the responsible committee on human experimentation or with the Helsinki Declaration of 1975 (as revised in 1983). The trial registered in a trial register (Eudra CT, registration no. 2008-005943-40).

Table 3.1 Study design.

\begin{tabular}{lcccccc}
\hline & Sequence 1 & Sequence 2 & Sequence 3 & Sequence 4 & Sequence 5 & Sequence 6 \\
\hline Period 1 & sublingual & sublingual & oral & oral & rectal & rectal \\
Period 2 & rectal & oral & sublingual & rectal & sublingual & oral \\
Period 3 & oral & rectal & rectal & sublingual & oral & sublingual \\
\hline
\end{tabular}

\section{Subjects}

Subjects between 18 and 65 years of age were recruited from a healthy volunteer database. Main exclusion criteria were as follows: regular drug use other than oral contraceptives, abnormal liver biochemistry or renal function, blood pressure $>160 / 100 \mathrm{mmHg}$ and abnormal electrocardiogram. Females with childbearing potential had to ensure effective contraception during the study period. Informed consent was obtained from all subjects.

\section{Treatment}

\section{Formulations}

Subjects received three different tacrolimus formulations: (1) an instant release capsule (Prograf, Astellas Pharma, Staines, UK) as oral formulation, (2) the powder content from capsule as sublingual formulation, and (3) a suppository containing the powder content from capsule. Suppositories were manufactured according to the Good Manufacturing Practice (GMP) by a GMP-certified hospital pharmacy. The suppositories comprised lactose monohydricum as fill-up on the basis of $\mathrm{H} 15$ witepsol.

Dose

The tacrolimus formulations were administered as a single dose. The oral capsule was given at a dose of $7 \mathrm{mg}$ (corresponding to $0.1 \mathrm{mg} / \mathrm{kg}$, the usual dose applied in clinical practice), the sublingual dose consisted of $3 \mathrm{mg}$ (in accordance with the earlier publication by Reams et al. ${ }^{5}$ ) and the suppository dose amounted $15 \mathrm{mg}$ (derived from our pilot study ${ }^{12}$ ). The rectal dose was higher than that used in case reports of patients with colitis, considering the presence of an intact mucosal barrier in healthy volunteers. 


\section{Study procedures}

Each subject received the above-mentioned formulations in a sequence according to their randomization seven days apart from each occasion at the same time point in the morning after an overnight fast. Consumption of alcohol and nicotine was prohibited during this phase. On each occasion an indwelling catheter (Braun Vasofix ${ }^{\circledR}$ Safety, Melsungen, Germany) was inserted into a forearm vein for the purpose of repeated venous blood sampling.

After supervised dosing, the subjects had to remain in a semi-recumbent position for three hours. The subjects received a standardized continental breakfast one hour after drug administration. Any adverse event during the study period was recorded.

For sublingual application, the powder content of the oral Prograf capsule was placed under the tongue for 15 minutes, during which period the subjects were not allowed to swallow. To establish a 'true' sublingual route, the subjects had to rinse their mouth with water (and spit this out) after the 15-minute period to prevent consecutive swallowing of residual tacrolimus powder. Any act of swallowing before this time point was recorded.

After rectal application a minimum interval of three hours had to be maintained between administration of the suppository and possible defecation (subjects were instructed to defecate before administration of the drug). In case of earlier defecation this intervention period had to be repeated on a later occasion, at least one week after former drug application.

\section{Blood sampling and assay}

Three $\mathrm{ml}$ of venous blood was drawn into an EDTA vacuum tube (Becton and Dickinson, Franklin Lakes NJ, USA). Blood was sampled pre-dose and during the first hour at 5, 10, 20, 30 and 60 minutes. Thereafter, samples were taken at 2, 4, 6, 8, 10, 12 and 24 hours post- dose. In case of rectal administration, samples were also taken at 36 and 48 hours after dosing.

The blood samples were stored at $4^{\circ} \mathrm{C}$ until further analysis between three days and three weeks later. Tacrolimus concentrations were determined by high-performance chromatography tandem mass spectroscopy (HPLC/MS/MS) as previously described. ${ }^{13}$ The routine assay in venous blood is linear from 1 to $300 \mu \mathrm{g} / \mathrm{l}$. Intra-assay precision and accuracy was 3.4\%, 2.2\%, 3.0\% and $102 \%, 94 \%$ and $94 \%$, respectively at 3.04, 6.23 and $13.0 \mu \mathrm{g} / \mathrm{l}(\mathrm{n}=6)$. Inter-assay precision and accuracy was $8.2 \%, 5.2 \%, 4.6 \%$ and $102 \%, 94 \%$, and $93 \%(n=9)$, respectively. Limit of quantification was $1.0 \mu \mathrm{g} / \mathrm{l}$. The laboratory successfully participates in the international tacrolimus proficiency testing scheme (www.bioanalytics.co.uk). 


\section{Genotyping}

Each subject was taken a venous blood sample at the screening visit for genetic analysis for Cytochrome P450 3A5 polymorphisms. For this purpose, genomic DNA was extracted from all subjects according to the manufacturers' instructions (Qiagen, Leusden, the Netherlands). Real-time polymerase chain reaction (PCR) fluorescence resonance transfer (FRET) assays were used for genotyping with the LightCycler (Roche Diagnostics, Almere, The Netherlands).

\section{Pharmacokinetic outcome parameters}

For all formulations the pharmacokinetic outcome parameters were: Time to reach maximum concentration $\left(T_{\max }\right)$, maximum blood concentration $\left(\mathrm{C}_{\max }\right)$ and area under the blood concentration-time curve $(A \cup C)$ from 0 time to 24 hours $\left(A \cup C_{0-24}\right)$. For rectal administration we measured the AUC from 0 time to 48 hours $\left(A \cup C_{0-48}\right) . C_{\max }$ and $T_{\max }$ were obtained from the raw data. The AUC was measured by the linear trapezoidal rule. $C_{\max }$ and AUC were 'dose-normalised' (Dn) by dividing the measured parameter through the respective dose. The relative bioavailability of the rectal formulation was estimated using the rectal to oral ratio of the natural log-transformed values for AUC and DnAUC. The inter-subject variability was quantified as the coefficient of variation (CV\%) according to:

$$
C V(\%)=100 \sqrt{e^{S_{B R}^{2}}-1}
$$

where $S_{B R}$ is the between subject standard deviation of the natural log-transformed values.

\section{Statistical analysis}

The study was powered to meet the EMA regulatory criteria for assessment of bioequivalence. ${ }^{14}$ According to these guidelines the number of evaluable subjects should not be less than 12. Considering possible drop-out and a three-period sixsequence crossover design we included 18 (i.e. $6 \times 3$ ) subjects in our study. Demographic data are reported as the mean and their standard deviations. Pharmacokinetic data are presented as the geometric mean and their $90 \%$ confidence interval. Data were natural log-transformed prior to statistical analysis and transformed back to the original scale for display of the results. The pharmacokinetic parameters under consideration were compared using ANOVA. The terms used in the ANOVA model were sequence, period and formulation. A p-value smaller than 0.05 was considered to be statistically significant. Statistical analysis was performed using SPSS 16.0 for Windows, release 16.0.1. 


\section{Results}

\section{Study population}

Eighteen healthy white subjects (six male and 12 female; age $39 \pm 16$ years, body mass index (BMI) $27.3 \pm 5.5 \mathrm{~kg} / \mathrm{m}^{2}$ ) were recruited and completed the study. Six female subjects used oral contraception during the study.

\section{Pharmacokinetics}

Regarding the single dose, three period crossover design, there was neither a confounding influence of the period nor of the sequence the subjects were randomized to, confirming the absence of carry-over effect between treatment periods (see Supplemental Table S3.1). A summary of the pharmacokinetic parameters for the oral and the rectal formulation is given in Table 3.2, while Figure 3.1 shows the individual blood concentration-time profiles after sublingual tacrolimus administration of all individual subjects.

Table 3.2 Pharmacokinetic parameters for the oral and the rectal formulation.

\begin{tabular}{|c|c|c|c|c|c|}
\hline \multirow[t]{2}{*}{ Parameter } & \multicolumn{2}{|l|}{ Oral } & \multicolumn{2}{|l|}{ Rectal } & \multirow[b]{2}{*}{$\begin{array}{c}\text { Ratio rectal/ora } \\
(90 \% \mathrm{Cl})\end{array}$} \\
\hline & $\begin{array}{c}\text { (Geom) mean } \\
(90 \% \mathrm{Cl})\end{array}$ & CV\% & $\begin{array}{c}\text { (Geom) mean } \\
(90 \% \mathrm{Cl})\end{array}$ & CV\% & \\
\hline $\begin{array}{l}T_{\max } \\
\text { (hours) }\end{array}$ & $1.58(1.37-1.80)$ & 44.7 & $4.23(3.35-5.10) *$ & 52.3 & $3.23(2.28-4.17)$ \\
\hline $\begin{array}{l}C_{\max } \\
(\mu g / I)\end{array}$ & $34.5(30.10-39.50)$ & 34.1 & $23.1(19.8-26.9)$ * & 38.7 & $0.67(0.57-0.79)$ \\
\hline $\begin{array}{l}\mathrm{DnC}_{\max } \\
(\mu \mathrm{g} / \mathrm{l} / \mathrm{mg})\end{array}$ & $4.9(4.3-5.6)$ & 34.1 & $1.5(1.3-1.8)^{*}$ & 38.7 & $0.31(0.27-0.37)$ \\
\hline $\begin{array}{l}\mathrm{AUC}_{0-24} \\
(\mu \mathrm{g} / \mathrm{h} / \mathrm{I})\end{array}$ & $194(170-222)$ & 33.0 & $292(233-365)$ * & 59.5 & $1.5(1.22-1.85)$ \\
\hline $\begin{array}{l}\text { DnAUC }_{0-24} \\
(\mu \mathrm{g} / \mathrm{h} / \mathrm{l} / \mathrm{mg})\end{array}$ & $28(24-32)$ & 33.0 & $19(16-24) *$ & 59.5 & $0.70(0.57-0.86)$ \\
\hline $\begin{array}{l}\mathrm{AUC}_{0-48} \\
(\mu \mathrm{g} / \mathrm{h} / \mathrm{l})\end{array}$ & - & - & 394 (309-504) & 65.2 & n.a \\
\hline $\begin{array}{l}\text { DnAUC }_{0-48} \\
(\mu \mathrm{g} / \mathrm{h} / \mathrm{l} / \mathrm{mg})\end{array}$ & - & - & $26(21-34)$ & 65.2 & n.a. \\
\hline $\begin{array}{l}\mathrm{T}_{1 / 2} \\
\text { (hours) }\end{array}$ & $24.0(19.5-28.5)$ & 36.85 & $22.8(21.6-24.0)$ & 27.23 & \\
\hline
\end{tabular}

Sublingual administration of $3 \mathrm{mg}$ of tacrolimus gave no detectable tacrolimus concentrations (i.e $>1 \mu \mathrm{g} / \mathrm{l}$ ) in 11 out of 18 patients. In seven subjects, tacrolimus concentrations were detected. In three out of these seven volunteers, a swallowing act was documented; these subjects had a $C_{\max }$ ranging between 1.3 and $22 \mu \mathrm{g} / \mathrm{l}$ and a $\mathrm{T}_{\max }$ of 2 hours. In the 4 cases without documented swallowing, the $\mathrm{C}_{\max }$ ranged from 
1.3 to $7.2 \mu \mathrm{g} / \mathrm{l}$, also with a $T_{\max }$ of 2 hours. All subjects reported to have difficulties with keeping the powder content in their mouth without swallowing for the required 15 minutes. Since tacrolimus concentrations after 'true' sublingual application (i.e. with the precautions taken to prevent gastro-intestinal absorption) were not consistent, no further statistical analysis was performed for the sublingual formulation.

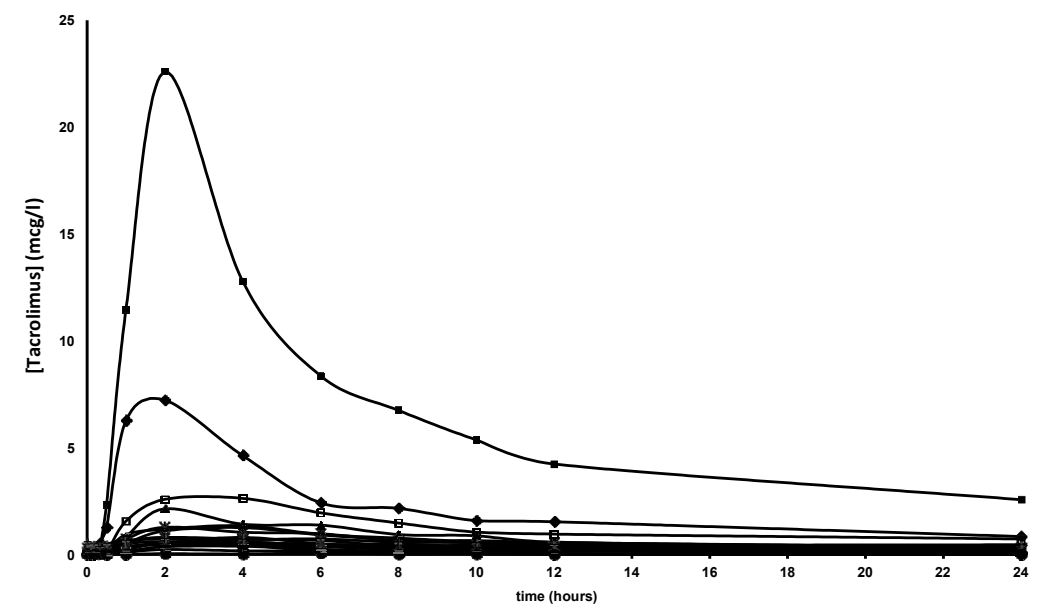

Figure 3.1 Individual blood concentration-time profiles after sublingual tacrolimus administration of all individual subjects.

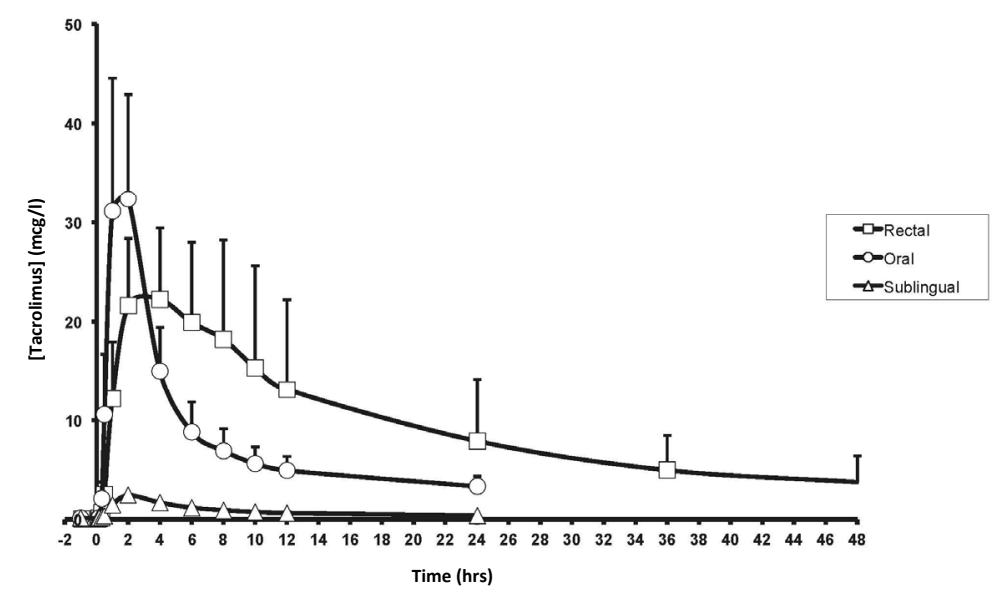

Figure 3.2 Average blood concentration-time curves of the three different formulations. 
Rectal application of tacrolimus showed clinically relevant systemic exposure in all volunteers (Figure 3.2). As listed in Table 3.2, dose-corrected AUCs were numerically lower for the suppository with a relative bioavailability of approximately $70 \%(90 \% \mathrm{Cl}$ $57-86 \%)$ after 24 hours referred to the oral formulation with a significantly lower $\mathrm{DnC}_{\max }(1.5 \mu \mathrm{g} / \mathrm{l} / \mathrm{mg}(\mathrm{Cl} 1.3-1.8 \mu \mathrm{g} / \mathrm{l} / \mathrm{mg})$ vs. $4.9 \mu \mathrm{g} / \mathrm{l} / \mathrm{mg}(\mathrm{Cl} 4.3-5.6 \mu \mathrm{g} / \mathrm{l} / \mathrm{mg}) ; \mathrm{p}<0.001)$ and a prolonged $\mathrm{T}_{\max }$ (4.2 hours ( $\mathrm{Cl} 3.3-5.1$ hours) vs. 1.58 hours $(\mathrm{Cl} 1.4-1.80$ hours); $\mathrm{p}<0.001)$. After 48 hours the AUC for the rectal formulation amounted $394 \mu \mathrm{g} / \mathrm{h} / \mathrm{I}$ $(\mathrm{Cl} 309-504 \mu \mathrm{g} / \mathrm{h} / \mathrm{l})$ with a trough level of $3.7 \mu \mathrm{g} / \mathrm{l}$. Eight of the 18 subjects have a biphasic absorption pattern as shown in Figure 3.3 for one representative subject. Volunteers with a biphasic absorption had a numerically higher $C_{\max }(26.8 \mu \mathrm{g} / \mathrm{l} \mathrm{vs}$. $22.5 \mu \mathrm{g} / \mathrm{l}$ ) and a statistically significantly higher (Dn)AUC $0-24$ (27 vs. $15 \mu \mathrm{g} / \mathrm{h} / \mathrm{l} / \mathrm{mg}$; $\mathrm{p}=0.02$ ). Rectal application of tacrolimus has a higher interpatient variation coefficient (CV) of drug exposure compared with the oral reference (Table 3.2). Volunteers with a biphasic absorption had a 13-27\% lower CV with regard to the different pharmacokinetic parameters compared with the subjects without a second concentration peak $(p<0.001)$.

The estimated elimination rate constant $\mathrm{K}_{\mathrm{e}}$ was 0.03 for both the oral and rectal formulation, with a respective corresponding $T_{1 / 2}$ of 24 hours ( $\mathrm{Cl} 19.5-28.5$ hours) and 22.8 hours ( $\mathrm{Cl} 21.6-24.0$ hours) (see supplemental content on the calculation of elimination half-life).

\section{Safety}

In total, 32 adverse events were reported during the study, none being serious. Most events were classified as 'mild' and five as 'moderate' (headache $n=3$, "migraine" $n=2)$. The most often reported adverse events were headache $(n=10)$ and paraesthesia at the application site $(n=7)$. Headache was mostly reported after rectal administration $(n=6)$. Paraesthesia at the application site occurred after sublingual $(n=5)$, and after rectal $(n=2)$ administration. For details see the Supplemental Table S3.2. 


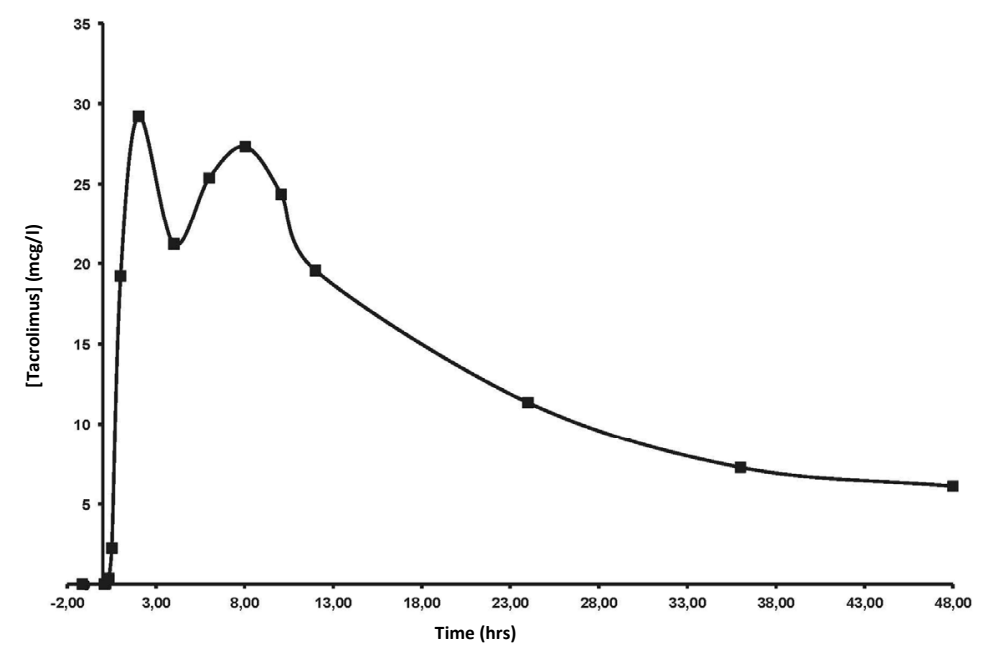

Figure 3.3 Example of a blood concentration-time profile of one representative subject with a biphasic absorption pattern.

\section{Discussion}

\section{Sublingual Tacrolimus}

To our knowledge this is the first 'adequately powered' pharmacokinetic study of sublingual and rectal formulations of tacrolimus with direct comparison of these formulations to the pharmacokinetics of the oral formulation in healthy volunteers. We first confirmed our earlier report that, contrary to earlier papers ${ }^{5,6}$, a single 'true' sublingual application (i.e. with the precautions taken to prevent gastro-intestinal absorption) of tacrolimus in a dose of $3 \mathrm{mg}$ (being comparable to the dose of $0.04 \mathrm{mg} / \mathrm{kg}$ applied in the Reams study) fails to produce consistent systemic exposure in healthy volunteers.

In accordance with the findings of Zhang et al. ${ }^{15}$ and Romero et al. ${ }^{8}$, the single subject in our series with clinically relevant exposure after sublingual dosing showed a concentration-time profile with a late $C_{\max }$ comparable to that of oral dosing, rather than to an early $C_{\max }$ as expected after sublingual absorption. The concentration-time profiles in Zahra's study show a similar pattern. ${ }^{6}$

Theoretically, the 15 minute sublingual administration might not have been long enough to allow for substantial absorption. However, $40 \%$ of the drug is dissolved within 15 minutes and we would expect fast absorption if true sublingual absorption had taken place of the current formulation with relevant tacrolimus levels. ${ }^{15,16}$ In 
clinical practice, we assume that it would not be feasible to keep the powder content under the tongue for more than 15 minutes. All subjects declared that keeping the drug content sublingual for 15 minutes without swallowing was very difficult, and indeed, in several subjects swallowing was observed.

Hence, we postulate that in Reams' and Zahra's report normal gastrointestinal absorption occurred after swallowing; the studies do not mention precautions that were taken to prevent this. Subjects in our study were instructed not to swallow during the first 15 minutes and to rinse their mouth afterwards with spitting the rinse solution under strict supervision. In addition, a substantial proportion of the subjects mentioned local paraesthesia as side-effect, underscoring the fact that tacrolimus (although in a small amount) had some local (side-)effect. Thus, in conclusion, we believe that single-dose sublingual tacrolimus application does not result into 'true' sublingual absorption, but that systemic exposure is probably a result of gastrointestinal absorption. Whether repetitive or higher doses might result in sublingual tacrolimus absorption remains unanswered, so far.

\section{Rectal administration}

The second goal of our study was to perform an adequately powered pharmacokinetic study of single dose rectal tacrolimus application through a suppository. All subjects had a clinically relevant systemic tacrolimus exposure. This has 2 consequences. First, this might be an alternative route for adequate systemic tacrolimus exposure (e.g. in solid organ transplant recipients). Second, rectal administration of tacrolimus with the goal to induce a local therapeutic effect in patients suffering from colitis may have systemic (side-)effects.

Van Dieren et al. reported local efficacy and detectable tacrolimus trough levels (maximum $5 \mu \mathrm{g} / \mathrm{l}$ ) after repeated rectal administration of 2-4 mg tacrolimus in patients with active colitis ${ }^{11}$ which implies systemic therapeutic levels with potential side effects. It should be considered that patients suffering from inflammatory bowel disease have a diminished mucosal barrier function resulting in increased and less predictable drug exposure compared with healthy volunteers.

Drug exposure after rectal application appeared to be more variable with almost twice as high inter-subject CV of the rectal formulation compared with the oral formulation. We assume that this increased inter-subject variability could be explained by the presence of two distinct subpopulations with different absorption patterns: a monophasic and a biphasic absorption. The reason for these different patterns is not completely understood. It could be that late defecation led to incomplete absorption resulting in a higher variability. By protocol, no subject defecated within the first three hours after dosing, but it is not documented whether the subjects did so on a later occasion. 
Another explanation for the second absorption peak could be saturation of the mucosal transport, combined with insufficient delivery of tacrolimus molecules due to bad dissolution (absence of water in the rectum) ${ }^{17}$, and subsequent drug absorption in more proximal bowel segments. Mucosal saturation may also be responsible for the later $\mathrm{T}_{\max }$ and the consistently elevated concentrations after rectal application (Figure 3.2), which indicates a prolonged absorption phase due to sustained transport over the mucosal barrier and slow release of tacrolimus from the rectal mucosal cells into the circulation. In clinical practice, this could allow for less frequent dosing, which may facilitate drug administration for people being unable or unwilling (e.g. children) to take the drug via the normal oral route.

Since we cannot exclude the possibility that the mucosal surface is a limitation to absorption of a large amount of drug, it might be that if the healthy volunteers were to have received a substantially lower rectal dose this might have resulted in better bioavailability. However, in our view $15 \mathrm{mg}$ is a relatively small amount of drug in comparison to other lipophilic drugs such as e.g. $500 \mathrm{mg}$ of acetaminophen, which is almost completely absorbed after rectal administration. The extent of absorption also depends on the surface over which the drug can spread after melting of the suppository and a large suppository will deliver a higher amount of drug than a small one. ${ }^{18}$ In this context we do not assume that the absorption of $15 \mathrm{mg}$ of tacrolimus is limited by its amount or the compartmental size, but by other mechanisms like mucosal pre-systemic metabolism of tacrolimus.

Mucosal enzymes like the cytochrome-P450, subfamily 3a5 (Cyp3A5), and the P-glycoprotein (P-gp) are major contributors to the pre-systemic metabolism of tacrolimus. ${ }^{19}$ Three out of 18 subjects were heterozygous carriers of the wild type Cyp3A5*1 genotype. However, these subjects did not show consistently identical absorption patterns so that we do not assume that the Cyp3A $5 * 3$ genotype plays a major role. The Cyp3A5 appears to be mainly localized in the proximal digestive tract $^{19-21}$ with decreasing catalytic activity downstream. ${ }^{22}$ This makes a contribution of this enzyme with regard to pre-systemic tacrolimus metabolism limited in case of rectal application.

The P-gp may play a more important role for active extrusion of the absorbed tacrolimus in the rectum, since P-gp mRNA levels are reported to increase longitudinally along the intestine, with highest level in the colon. ${ }^{21,23}$ Single Nucleotide Polymorphisms (SNPs) of the P-gp may be associated with a decreased protein expression. ${ }^{24}$ We were not able to confirm this hypothesis since we did not determine P-gp SNPs.

\section{Limitations}

We achieved our goal to determine if rectal tacrolimus administration results in systemic exposure and to compare the pharmacokinetics to orally administered 
tacrolimus. However, because at our latest sample time point (48 hours) tacrolimus blood concentrations were still $3.7 \mu \mathrm{g} / \mathrm{l}$ after rectal administration, sampling in future pharmacokinetic studies should be prolonged up to at least 72 hours for better evaluation of the elimination phase and bio-availability.

We are aware of the fact that only the relative bioavailability compared with the oral formulation can be given. This is due to the nature of the study; on the one hand, it was meant to provide us, for the first time, with firm pharmacokinetic data on alternative tacrolimus formulations, but on the other hand, it was meant to prove the concept whether or not these formulations result in significant exposure, at all. In this light, we argued that comparison with the oral formulation would suffice. Given that we found a clinically relevant outcome with potential use of rectal tacrolimus formulations, future studies should provide us with data on the absolute bioavailability and thus an intravenous arm will have to be added.

Another issue is the different doses used for each formulation. Due to these different doses, blood concentrations had to be dose-corrected, making direct comparison less clear. Nevertheless, these limitations do not undermine our conclusions.

\section{Conclusion and future directions}

In conclusion, single sublingual dosing of $3 \mathrm{mg}$ tacrolimus in healthy volunteers does not result in consistent clinically relevant systemic exposure, provided swallowing of the tacrolimus powder (with subsequent gastrointestinal resorption) is prevented. It is not precluded, however, that repetitive and/or escalated dosing or other formulations of tacrolimus (e.g. intravenous solution or solid dispersions ${ }^{25}$ ) could result in significant drug exposure after sublingual exposure. This is in line with the finding that one in three subjects experienced local paraesthesia after sublingual dosing. This has to be explored further.

On the contrary, rectal application of a single dose of tacrolimus by means of a suppository gives consistent clinically relevant tacrolimus exposure and, therefore, may represent an alternative formulation under circumstances that normal gastrointestinal absorption is not feasible. Future bioequivalence and phase II studies with repetitive ascending dosing should establish the optimal doses and dosing frequencies, which would also supply us with more data on dose proportionality. These studies should then also be carried out in relevant patient populations (e.g. solid organ transplant recipients or patients with (distal) colitis). Until then, when applying tacrolimus by suppository, one has to be aware of significant exposure including systemic (side-) effects apart from the local/topical efficacy and therapeutic drug monitoring will be required. 


\section{References}

1. Cox TH, Baillie GM, Baliga P. Bradycardia associated with intravenous administration of tacrolimus in a liver transplant recipient. Pharmacotherapy 1997;17:1328-1330.

2. Takahashi S, Sugimoto K, Hishikawa S, Mizuta K, Fujimura A, Kawarasaki H. Recurrence of hepatic artery thrombosis following acute tacrolimus overdose in pediatric liver transplant recipient. Pediatric Transplant 2005;9:809-812.

3. Collin C, Boussaud V, Lefeuvre S, Amrein C, Glouzman AS, Havard L, Billaud EM, Guillemain R. Sublingual tacrolimus as an alternative to intravenous route in patients with thoracic transplant: a retrospective study. Transplant Proc 2010;42:4331-4337.

4. Nicolai S, Bunyavanich S. Hypersensitivity reaction to intravenous but not oral tacrolimus. Transplantation 2012;94:e61-e63.

5. Reams D, Rea J, Davis D, Palmer S. Utility of sublingual tacrolimus in cystic fibrosis patients after lung transplantation. J Heart Lung Transplant 2001;20:207-208.

6. Nasiri-Toosi Z, Dashti-Khavidaki S, Nasiri-Toosi M, Khalili H, Jafarian A, Irajian H, Abdollahi A, Sadrai S. Clinical pharmacokinetics of oral versus sublingual administration of tacrolimus in adult liver transplant recipients. Exp Clin Transplant 2012;10:586-591.

7. van de Plas A, Dackus J, Christiaans MH, Stolk LM, van Hooff JP, Neef C. A pilot study on sublingual administration of tacrolimus. Transpl Int 2009;22:358-359.

8. Romero I, Jimenez C, Gil F, Escuin F, Ramirez E, Fudio S, Borobia A, Carcas A. Sublingual administration of tacrolimus in a renal transplant patient. J Clin Pharm Ther 2008;33:87-89.

9. Sakai M, Hobara N, Hokama N, Kameya H, Ohshiro S, Sakanashi M, Saitoh H. Increased bioavailability of tacrolimus after rectal administration in rats. Biol Pharm Bull 2004;27:1480-1482.

10. Lawrance IC, Copeland TS. Rectal tacrolimus in the treatment of resistant ulcerative proctitis. Aliment Pharmacol Ther 2008;28:1214-1220.

11. van Dieren JM, van Bodegraven AA, Kuipers EJ, Bakker EN, Poen AC, van Dekken H, Nieuwenhuis EE, van der Woude CJ. Local application of tacrolimus in distal colitis: feasible and safe. Inflam Bowel Dis 2009;15:193-198.

12. van de Plas A, Dackus J, Christiaans MH, Stolk LM, van Hooff JP, Neef C. Pharmacokinetics of sublingual and rectal administration of tacrolimus: a pilot study. Pharmaceutisch Weekblad 2008;2: 66-68.

13. Hoogtanders K, van der Heijden J, Christiaans M, Edelbroek P, van Hooff JP, Stolk LM. Therapeutic drug monitoring of tacrolimus with the dried blood spot method. J Pharm Biomed Anal 2007;44: 658-664.

14. Note for Guidance on the Investigation of Bioavailability and Bioequivalence (NfG on BA BE). In, edAgency EM, London, 2010.

15. Zhang $\mathrm{H}$, Zhang J, Streisand JB. Oral mucosal drug delivery: clinical pharmacokinetics and therapeutic applications. Clin Pharmacokinet 2002;41:661-80.

16. Petan JA, Undre N, First MR, Saito K, Ohara T, Iwabe O, Mimura H, Suzuki M, Kitamura S. Physiochemical properties of generic formulations of tacrolimus in Mexico. Transplant Proc 2008;40: 1439-1442.

17. Venkataramanan R, Jain A, Warty VS, Abu-Elmagd K, Alessiani M, Lever J, Krajak A, Flowers J, Mehta S, Zuckerman S, et al. Pharmacokinetics of FK 506 in transplant patients. Transplant Proc 1991;23: 2736-2740.

18. van Hoogdalem E, de Boer AG, Breimer DD. Pharmacokinetics of rectal drug administration, Part I. General considerations and clinical applications of centrally acting drugs. Clin Pharmacokinet 1991;21:11-26.

19. Zhang Y, Benet LZ. The gut as a barrier to drug absorption: combined role of cytochrome P450 $3 A$ and P-glycoprotein. Clin Pharmacokinet 2001; 40: 159-168.

20. Paine MF, Hart HL, Ludington SS, Haining RL, Rettie AE, Zeldin DC. The human intestinal cytochrome P450 "pie". Drug Metab Dispos 2006;34:880-886. 
21. Thorn M, Finnstrom N, Lundgren S, Rane A, Loof L. Cytochromes P450 and MDR1 mRNA expression along the human gastrointestinal tract. Br J Clin Pharmacol 2005;60:54-60.

22. Wacher VJ, Silverman JA, Zhang Y, Benet LZ. Role of P-glycoprotein and cytochrome P450 $3 \mathrm{~A}$ in limiting oral absorption of peptides and peptidomimetics. J Pharm Sci 1998;87:1322-1330.

23. Fojo AT, Ueda K, Slamon DJ, Poplack DG, Gottesman MM, Pastan I. Expression of a multidrugresistance gene in human tumors and tissues. Proc Natl Acad Sci U S A 1987;84:265-269.

24. Hoffmeyer S, Burk O, von Richter O, Arnold HP, Brockmoller J, Johne A, Cascorbi I, Gerloff T, Roots I, Eichelbaum M, Brinkmann U. Functional polymorphisms of the human multidrug-resistance gene: multiple sequence variations and correlation of one allele with P-glycoprotein expression and activity in vivo. Proc Natl Acad Sci U S A 2000;97:3473-3478.

25. Srinarong P, Pham BT, Holen M, van der Plas A, Schellekens RC, Hinrichs WL, Frijlink HW. Preparation and physicochemical evaluation of a new tacrolimus tablet formulation for sublingual administration. Drug Dev Ind Pharm 2012;38(4):490-500. 


\section{Supplemental material}

Table S3.1 Influence of sequence, period and formulation on pharmacokinetic outcome parameters (ANOVA analysis, p-values).

\begin{tabular}{lccc}
\hline Dep/factor & Sequence & Period & Treatment \\
\hline$T_{\text {max }}($ hours $)$ & 0.756 & 0.856 & $<0.001$ \\
$C_{\text {max }}(\mu \mathrm{g} / \mathrm{l})$ & 0.064 & 0.288 & 0.001 \\
$\operatorname{DnC}_{\text {max }}(\mu \mathrm{g} / \mathrm{l} / \mathrm{mg})$ & 0.064 & 0.292 & $<0.001$ \\
$\mathrm{AUC}_{0-24}(\mu \mathrm{g} / \mathrm{h} / \mathrm{l})$ & 0.182 & 0.585 & 0.007 \\
$\operatorname{DnAUC}_{0-24}(\mu \mathrm{g} / \mathrm{h} / \mathrm{l} / \mathrm{mg})$ & 0.138 & 0.586 & 0.015 \\
$\mathrm{AUC}_{0-48}(\mu \mathrm{g} / \mathrm{h} / \mathrm{l})$ & 0.187 & 0.585 & 0.006 \\
DnAUC $_{0-48}(\mu \mathrm{g} / \mathrm{h} / \mathrm{l} / \mathrm{mg})$ & 0.187 & 0.585 & 0.042 \\
AUC $_{0-72}(\mu \mathrm{g} / \mathrm{h} / \mathrm{l})$ & 0.239 & 0.512 & 0.003 \\
DnAUC $_{0-72}(\mu \mathrm{g} / \mathrm{h} / \mathrm{l} / \mathrm{mg})$ & 0.239 & 0.512 & 0.074 \\
AUC $_{0-\text { inf }}(\mu \mathrm{g} / \mathrm{h} / \mathrm{l})$ & 0.324 & 0.341 & 0.002 \\
DnAUC $_{0-\text { inf }}(\mu \mathrm{g} / \mathrm{h} / \mathrm{l} / \mathrm{mg})$ & 0.324 & 0.341 & 0.103 \\
\hline
\end{tabular}

Table S3.2 Overview of adverse events.

\begin{tabular}{llll}
\hline Adverse event & oral & rectal & sublingual \\
\hline $\begin{array}{l}\text { General disorders } \\
\text { fatigue }\end{array}$ & 0 & 2 & 0 \\
$\quad$ dizziness & 0 & 1 & 1 \\
$\quad \begin{array}{l}\text { Administration site conditions } \\
\quad \text { paraesthesia }\end{array}$ & 1 & 2 & 5 \\
Nervous sytem disorders & & & \\
$\quad$ headache & 3 & 6 & 1 \\
$\quad$ migraine & 1 & 2 & 1 \\
tremor & 1 & 1 & 1 \\
$\quad$ paraesthesia \# & 0 & 1 & 0 \\
$\quad$ dysaesthesia & 0 & 1 & 0 \\
feeling of temperature change & 0 & 1 & 0 \\
\hline
\end{tabular}

\# excluding paraesthesia at administration site. 
Supplemental Content: Calculation of $\mathrm{AUC}_{0-\infty}$ and elimination Half life

To have a more thorough indication of the elimination phase we extrapolated tacrolimus concentrations with 12-hour intervals until 72 hours by multiplying the last measured concentration with the elimination rate constant:

$$
C_{t}=k_{e} \cdot C_{0}
$$

Where $C_{t}$ is the concentration to be extrapolated for time point $t, k_{e}$ is the elimination rate constant and $C_{0}$ is the last measured/extrapolated concentration. The elimination rate constant $\mathrm{K}_{\mathrm{e}}$ was determined by log-linear regression. The AUCO- $\infty$ was then calculated by:

$$
\mathrm{AUC}_{0-\infty}=\mathrm{AUC}_{0-72}+\frac{\mathrm{C}_{\mathrm{t}}}{\beta}
$$

where $C_{t}$ is the last measurable concentration above the lower limit of quantification and $\beta$ is the slope of the elimination phase obtained by linearising the last logtransformed concentrations vs. time. ${ }^{23}$ The elimination half life $\left(T_{1 / 2}\right)$ was calculated according to:

$$
\mathrm{T}_{1 / 2}=\operatorname{Ln}(2) / \mathrm{k}_{\mathrm{e}}
$$





\section{Chapter 4}

A limited sampling strategy to estimate exposure of oncedaily modified release tacrolimus in renal transplant recipients using linear regression analysis and comparison with Bayesian population pharmacokinetics in different cohorts

F. Stifft, F. Vandermeer, C. Neef, S. van Kuijk, M. Christiaans

European Journal of Clinical Pharmacology DOI 10.1007/s00228-019-02814-x 


\section{Abstract}

\section{Purpose}

Tacrolimus has a narrow therapeutic window. Measuring trough level $\left(C_{0}\right)$ as surrogate for drug exposure (AUC) in renal transplant recipients has limitations. Therefore, Limited Sampling Strategies (LSS's) have been developed. For the newer modified release once-daily formulation (TaC QD) LSS's are based on either linear regression analysis (LRA) or population pharmacokinetics with Maximum a posteriori Bayesian (MAPB) estimation. The predictive performances of both methods were compared, also to LSS's as described in literature.

\section{Methods}

LSS's (maximally three sampling time points) were developed for Tac QD from full 24-hour sampling by LRA in 27 white, stable renal transplant recipients. Performance for accuracy (Mean Absolute Prediction Error <10\%) and precision (Root Mean Squared Error <15\%) was quantified also after MAPB estimation in two independent groups (early and late post-transplant, $n=12$ each).

\section{Results}

LRA determined a single eight hours post-dose measurement $\left(C_{8}\right)$ to fulfil predefined criteria for accuracy (MAPE 3.41\%) and precision (RMSE 4.28\%). The best LSS contained $\mathrm{C}_{2}, \mathrm{C}_{8}$ and $\mathrm{C}_{12}$ for the stable (MAPE $2.42 \%$, RMSE $3.1 \%$ ) and the early posttransplant group (MAPE 2.46\%, RMSE 3.14\%). LRA did not include $\mathrm{C}_{0}$ for any LSS, unless it was forced into the model. MAPB estimation showed similar performance.

\section{Conclusions}

In renal transplant patients sampling in the elimination phase $\left(C_{8}\right)$ accurately predicted Tac QD exposure, contrary to $C_{0}$. The 3-point sampling $C_{2}, C_{8}$ and $C_{12}$ had the best performance and is also valid early post-transplant. These LSS's were similarly predictive with MAPB estimation. Dried Blood Spot could facilitate late sampling in clinical practice. 


\section{Introduction}

Tacrolimus is a pivotal immunosuppressant in solid organ transplantation for prevention of transplant rejection. ${ }^{1}$ Due to its narrow therapeutic window and high variability, therapeutic drug monitoring (TDM) is advised for optimal drug dosing. ${ }^{2}$ The area under the concentration time curve (AUC) (drug exposure) correlates with efficacy and side effects. ${ }^{3,4}$ In a consensus report on the immediate release twice-daily formulation of tacrolimus (Tac BID), an AUC from 0-12 hours $\left(A \cup C_{0-12}\right.$ ) has been denoted to be the best pharmacokinetic (PK) marker for overall drug exposure. ${ }^{2}$ In clinical practice, TDM with $\mathrm{AUC}_{0-12}$ measurement is not feasible and monitoring is routinely based on tacrolimus trough levels $\left(C_{0}\right)$. However, several authors report a moderate predictive value of the $C_{0}$ for the AUC for Tac BID. ${ }^{5-7}$ Limited Sampling Strategies (LSS's) consisting of two to three sampling points were validated and have shown to better predict AUC for Tac BID. ${ }^{8}$

A once-daily modified-release formulation of tacrolimus (Tac QD; Advagraf, Astellas Pharma, Staines, UK) has been introduced in the market. In PK studies, drug exposure to this formulation has been expressed as the AUC from 0-24 hours $\left(A \cup C_{0-24}\right){ }^{9}$ Tac QD $\mathrm{C}_{0}$ has been reported to correlate with the $\mathrm{AUC}_{0-24}$ and is recommended for TDM. ${ }^{9-11}$ For Tac QD, it has also been postulated that $C_{0}$ could only be considered a fair, but not a very good, surrogate marker of the overall exposure. ${ }^{12}$ In LSS studies on Tac QD, sampling times mostly have been constrained to the first hours after dosing for reasons of patient convenience and clinical feasibility. ${ }^{13-15}$ It is unknown whether later sampling would allow more accurate prediction of Tac QD exposure. In addition, different methods are used for LSS development. Whereas some papers describe a model based on Linear Regression Analysis (LRA) ${ }^{8,16}$, a French group describes a model on the basis of population PK analysis. ${ }^{13,14}$

The aim of our study was to develop a LSS for Tac QD in our (mainly white) renal transplant population, on the basis of LRA over the complete 24-hour dose interval in the maintenance phase ( $>6$ months post-transplant) and to validate it in 2 independent groups: stable ( $>6$ months post-transplant) and early ( 2 weeks posttransplant). At the same time, the raw PK data of the stable index group would be used for estimating population PK parameters. The best predicting time points from LRA would then be used in our MAPB model. The predictive performance for estimating the $A_{U-24}$ by means of LRA and MAPB-estimation would then be compared through validation in independent populations, as with (optimal) sampling time points described in literature. ${ }^{13,14,16}$ 


\section{Patients and methods}

\section{Patient groups}

The patient groups consisted of three independent groups of renal transplant recipients:

1. The index group consisted of 27 patients (17 male, 10 female) from our centre participating in an international multicentre study, investigating the long-term efficacy and safety of Tac $\mathrm{QD}^{17}$ who provided informed consent for a sub-study, approved by the local medical ethical board (MEC). ${ }^{18}$ Their mean age was 49 years (range 31-63). All patients were white, with the exception of one Asian male. These patients had been converted before from Tac BID to Tac QD at least 6 months after transplantation and had stable graft function before inclusion in this local study. The patients were tested by a 10 -point steady state 24 -hour blood concentration-time profile. For details of this study we refer to the Chapter $5 .^{18}$

2. Validation group 1 . This group consisted of 12 recipients ( 8 male, 4 female, all white) of our centre participating in an international, multi-centre, open label, prospective, conversion PK study in stable renal transplant recipients at least 6 months after transplantation. ${ }^{19}$ In this study, transplant patients had been converted from Tac BID to Tac QD and provided two 17-sample 24-hour blood concentrationtime profiles for each formulation in steady state.

3. Validation group 2 consisted of 12 French kidney transplant patients ( 3 male, 9 female) that had participated in a pharmacokinetic phase II trial ${ }^{20}$ tested at week 2 and 6 post-transplant as described by Saint-Marcoux et al.. ${ }^{14}$ The sponsor of that study kindly provided the raw PK data.

None of the patients in the index group or validation group 1 used medications interacting with the pharmacokinetics of Tac $Q D^{18,19}$, and all patients took the drug in a fasted state, i.e. at least one hour before a meal. In validation group 2 (patients from the Saint-Marcoux paper), none of the potentially interacting co-medication was frequent enough to emerge as a covariate in the model. Also, the Bayesian estimators were strong enough with 3 time-points to overcome the variability brought in by Сyp3A polymorphisms and drug-drug interactions (personal communication). An overview of the study populations and basic demographics is given in Table 4.1.

\section{Blood sampling}

Tacrolimus concentrations were determined from venous blood samples taken by phlebotomy from each patient in all three patient groups. Since the index population and the validation population had different sampling frequencies (10 samples and 17 samples, respectively) we used the ten common sampling times from each group. 
These were: approximately 5 minutes prior to dosing $\left(C_{0}\right)$ and at $1\left(C_{1}\right), 2\left(C_{2}\right), 3\left(C_{3}\right)$, $4\left(C_{4}\right), 6\left(C_{6}\right), 8\left(C_{8}\right), 10\left(C_{10}\right), 12\left(C_{12}\right)$ and $24\left(C_{24}\right)$ hours post-dose.

Table 4.1 Subject demographics.

\begin{tabular}{|c|c|c|c|}
\hline Reference & Stifft et al. ${ }^{18}$ & Van Hoof et al. ${ }^{19}$ & Saint Marcoux et al. ${ }^{14}$ \\
\hline Number of patients & 27 & 12 & 12 \\
\hline Kidney transplant phase & $\begin{array}{l}\text { Maintenance } \\
\text { (>6 months) }\end{array}$ & $\begin{array}{l}\text { Maintenance } \\
\text { (>6 months) }\end{array}$ & $\begin{array}{l}\text { De novo } \\
\text { (6 weeks) }\end{array}$ \\
\hline \multicolumn{4}{|l|}{ Gender } \\
\hline Male & 17 & 9 & 3 \\
\hline Female & 10 & 3 & 9 \\
\hline \multicolumn{4}{|l|}{ Race } \\
\hline white & 26 & 12 & 12 \\
\hline other & 1 (Asian) & 0 & 0 \\
\hline \multicolumn{4}{|l|}{ Age (years) } \\
\hline Mean & 49 & 55 & Unknown $^{\mathrm{a}}$ \\
\hline range & $31-63$ & $21-64$ & \\
\hline \multicolumn{4}{|l|}{ Days since transplantation } \\
\hline \multirow[t]{2}{*}{ range } & 3185 & 1389 & 14 and 42 \\
\hline & $1968-5694$ & $546-2209$ & n.a. ${ }^{b}$ \\
\hline \multicolumn{4}{|l|}{ Weight (kg) } \\
\hline Mean & 78 & 76 & Unknown $^{\mathrm{a}}$ \\
\hline range & $58-100$ & $56-116$ & \\
\hline \multicolumn{4}{|l|}{ Daily dose Tac QD (mg/kg) } \\
\hline Mean & 0.06 & 0.05 & Unknown $^{\mathrm{a}}$ \\
\hline range & $0.01-0.2$ & $0.02-0.11$ & \\
\hline
\end{tabular}

${ }^{a}$ Parameter of this subset of patients was not mentioned in Reference. ${ }^{14}$ Mean age (range) of the entire study population as published in reference ${ }^{20}$ was 44 (19-66) years; ${ }^{b}$ n.a. $=$ not applicable since PK profiles were taken on day 14 and day 42 , respectively, after transplant according to study protocol. ${ }^{20}$

\section{Sample handling and tacrolimus assay}

Blood samples were frozen within 1 hour of withdrawal and stored at $-20^{\circ} \mathrm{C}$ until shipping to a central laboratory for analysis, as described earlier. ${ }^{17,19,20}$ Whole blood tacrolimus concentrations were determined using ultra performance liquid chromatography mass spectrometry (UPLC-MS/MS) in two central laboratories: for the index population and validation group 2 this was Covance Laboratories Ltd, Harrogate, UK with a lower limit of quantification of $0.1 \mu \mathrm{g} / \mathrm{l}$. For the validation group 1 this was Farmovs-Parexel, Bloemfontein, South Africa with a lower limit of quantification of $0.059 \mu \mathrm{g} / \mathrm{l}$. Analyses of PK samples followed the Principles of Good Laboratory Practice.

\section{Sample size}

Due to the nature of our study no formal sample size calculation was performed. Based on previous data on LSS with similar studies, the number of 27 available profiles 
in our study is in line with earlier reports on the development of a population kinetic model. ${ }^{13,14,21}$ The dataset of 27 patients also exceeded the minimum sample size for bioequivalence $(\mathrm{N}=12)$ research as stated in current guidelines. ${ }^{22}$

\section{Pharmacokinetic modelling and validation}

\section{Linear regression analysis}

The non-compartmental $\mathrm{AUC}_{0-24}$ was determined using the linear trapezoidal rule. Multiple linear regression was used to determine the linear regression coefficient $(R)$ of the evolving LSS's up to three time points over the full 24-hour range of the PK-profile. In addition, the linear regression coefficient of multiple determination $\left(R^{2}\right)$ was calculated for expression of strength of association between the predicted and the actual $A \cup C_{0-24}$. The multilinear regression analysis for prediction of the $\mathrm{AUC}_{0-24}$ was performed by entering the Tacrolimus full blood concentrations at $t=0, t=2, t=4, t=8$, $t=12, t=14, t=16$ and $t=24$ hours post dose, using both stepwise forward selection and backward elimination techniques. Since it is common practice to include a trough level for tacrolimus monitoring, we performed a second run of regression analyses after forced entering of the trough level $\mathrm{C}_{0}$ (as Niioka did ${ }^{16}$ ) and $\mathrm{C}_{24}$, if the first regression model did not already include this. In addition, we also entered the time points as described by Niioka et al. and Saint-Marcoux et al. into the regression analysis. The search for the best fitting regression model containing only significant associations was done. The final model had to have a $p$-value $<0.05$. For being included in the final model, individual parameters had to have a $p$-value $<0.05$ in forward selection technique, or $p$-value $<0.10$ in the backward elimination technique. Analyses were performed using SPSS for Windows, version 20 (IBM statistics, USA).

\section{Maximum A Posteriori Bayesian estimation}

For our index group, we used a population PK model, developed using the KINPOP module in MW \Pharm 3.81 (Mediware, Czech Republic). KINPOP uses an iterative two-stage Bayesian analysis (ITSB). The ITSB analysis used in MW $\backslash$ Pharm 3.81 is a method of reliably estimating population PK parameters, which has been shown to perform well in rich datasets and established in the field of population pharmacokinetics ${ }^{23,24}$

We chose, as others ${ }^{13,15,25}$, a two-compartment PK model with first order absorption and elimination and with a lag time. Due to the first post-dose blood sample being at one hour post-dose, the information on the lag time was limited in the data. The lag time was estimated as a common parameter for all patients without inter-patient variability (dissolution of the capsule). Residual error was described by an exponential error model and this was calculated along with the PK parameters. The initial estimates were derived from the papers by Woillard, relating to distribution and 
elimination, and from Xue, relating to absorption and lag time, with a $50 \%$ coefficient of variation. $^{15,25}$

Although our software package used for population kinetic model development does not allow direct identification of sub-populations in PK parameters (e.g. fast and slow metabolizers), identifying subpopulations by post-analysis visual inspection of the frequency histograms of individual population kinetic parameters did not reveal any subpopulations (data not shown).

We used two different methods for MAPB estimation of the $\mathrm{AUC}_{0-24}$. First, the sampling times derived from the LRA were applied in our model. Second, sampling times in our model for MAPB estimation were calculated by D-optimality. ${ }^{26}$ In $D$-optimality, the determinant of the Fisher information matrix has been maximized. A detailed description of $D$ optimality is provided in the supplementary Appendix $A$. The appropriate calculations have been made using custom built software based on the VB.net programming language implemented in Microsoft Visual Studio 2008. As for LRA, we tested sampling time intervals of the complete dosing interval of 0 to 24 hours post-dose.

\section{Predictive performance}

For LRA- and MAPB-estimation, the predicted $\mathrm{AUC}_{0-24}$ were validated in independent populations, as defined above. In the MAPB estimation, the PK parameter for which the predictive performance is calculated is "Apparent Clearance from the central compartment" $(\mathrm{Cl} / \mathrm{F})$. In steady state, $\mathrm{Cl} / \mathrm{F}$ is directly related to AUC of the dosing interval:

$$
\frac{\text { Clearance }}{F}=\frac{\text { Dose }}{A U C_{0-24}}
$$

For each individual patient in the validation groups, the individual PK parameters and AUC are calculated using only the sampling times of interest, as determined by LRA. For validation group 2, only the raw PK data were available. For these patients, a dosing schedule of $5 \mathrm{mg}$ tacrolimus once daily was assumed with weight of $70 \mathrm{~kg}$ for each patient.

The calculated AUC, both from LRA as from MAPB, were then compared to the trapezoidal AUC of the full PK profile. Validation of the data occurred as described by Sheiner and Beal. ${ }^{27}$ The percentage of the mean prediction error (MPE\% = prediction bias) was calculated as:

$$
M P E \%=\frac{1}{n} \sum_{i=1}^{n}\left(\frac{A U C_{\text {pred,i }}-A U C_{\text {actual }, i}}{A U C_{\text {actual }, i}}\right) \times 100
$$


where $A \cup C_{\text {pred, } i}$ is the predicted $A \cup C$ of subject $i$ and $A U C_{a c t u a l, i}$ is the reference or actual AUC of subject $i$. The prediction accuracy was measured by the percentage of the mean absolute prediction error (MAPE\%):

$$
M A P E \%=\frac{1}{n} \sum_{i=1}^{n}\left(\frac{\left|A U C_{p r e d, i}-A U C_{a c t u a l, i}\right|}{A U C_{a c t u a l, i}}\right) \times 100
$$

The root mean squared error (RMSE\%) was used as a measure for precision and calculated as:

$$
R M S E \%=\sqrt{\frac{1}{n} \sum_{i=1}^{n}\left(\frac{A U C_{p r e d, i} \mid A U C_{a c t u a l, i}}{A U C_{a c t u a l, i}}\right)^{2}} \times 100
$$

We set an absolute prediction error (MAPE\%) of less than $10 \%$ and a RMSE\% of less than $15 \%$ with a prediction bias (MPE\%) of less than $5 \%$ to be required for valid prediction of the $\mathrm{AUC}_{0-24}$ in clinical practice. ${ }^{8,16}$ We also considered the number (and percentage) of patients with accurate prediction of the AUC (i.e. MAPE\% $<10 \%$ ) by each strategy. In addition, the agreement between the predicted and the measured $\mathrm{AUC}_{0-24}$ was determined by Bland Altman analysis. ${ }^{28}$

For our predictive performance analysis of the D-optimal sampling times we decided to use simulated patients. The predictive performance of all combinations of the up to maximum 5 D-optimal sampling times was tested in a simulated dataset of 500 patients, created by Latin Hypercube (LH) sampling from the population pharmacokinetic model of the index group. This is a method of sampling from a (log) normal distribution. LH sampling divides the cumulative distribution function (CDF) of a distribution into $\mathrm{N}$ even parts. A random sample is taken from each part of the CDF. This approach guarantees the entire CDF is used and evenly sampled to create the dataset. Each collection of LH drawn PK parameters is randomly paired to create simulated patients.

\section{Results}

\section{Pharmacokinetics}

The ranges (and median) of AUC's for all patient groups are presented as box-plot in Figure 4.1. In the index population the mean $\mathrm{AUC}_{0-24}$ was $204 \mathrm{\mu g} \cdot \mathrm{h} / \mathrm{l}$ (median $\mathrm{AUC}_{0-24}$ was $202 \mu \mathrm{g} \cdot \mathrm{h} / \mathrm{l}$, range 140-264 $\mu \mathrm{g} \cdot \mathrm{h} / \mathrm{l}$ ) with a corresponding mean $\mathrm{C}_{0}$ of $6.2 \mu \mathrm{g} / \mathrm{l}$ (median $6.2 \mu \mathrm{g} / \mathrm{l}$, range $4.2-8.2 \mu \mathrm{g} / \mathrm{l}$ ). In the maintenance population (validation group 1), the mean $\mathrm{AUC}_{0-24}$ was $206 \mu \mathrm{g} \cdot \mathrm{h} / \mathrm{l}$ (median $220 \mu \mathrm{g} \cdot \mathrm{h} / \mathrm{l}$, range 106-289 $\mu \mathrm{g} \cdot \mathrm{h} / \mathrm{l}$ ) with a corresponding mean $\mathrm{C}_{0}$ of $5.9 \mu \mathrm{g} / \mathrm{l}$ (median $6.2 \mu \mathrm{g} / \mathrm{l}$, range 
2.7-8.3 $\mu \mathrm{g} / \mathrm{l})$. On day 14 in the early post-transplant population (validation group 2), the mean $\mathrm{AUC}_{0-24}$ was $344 \mu \mathrm{g} \cdot \mathrm{h} / \mathrm{l}$ (median $356 \mu \mathrm{g} \cdot \mathrm{h} / \mathrm{l}$, range 172-542 $\mu \mathrm{g} \cdot \mathrm{h} / \mathrm{l}$ ) with a mean $\mathrm{C}_{0}$ of $9.7 \mu \mathrm{g} / \mathrm{l}$ (median 10,6 $\mathrm{\mu g} / \mathrm{l}$, range $4.4-14 \mu \mathrm{g} / \mathrm{l}$ ). On day 42 these values were similar (data not shown).

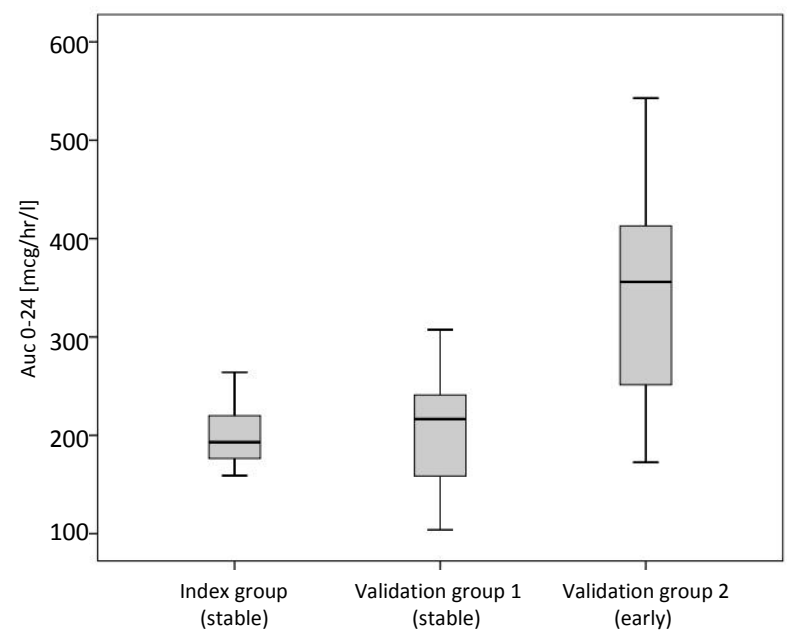

Figure 4.1 Boxplot of the $\mathrm{AUC}_{0-24}$ of the Index group and the validation groups 1 and 2 . The band inside the box shows the median value, whereas the bottom and top of the box represent the first and third quartiles, respectively. The small vertical lines at the end of the whiskers denote the lowest and highest value that are still within 1.5 times the interquartile range (IQR).

\section{Linear regression analysis}

Of all single time point measurements, the $C_{8}$ value had the highest correlation coefficient $\left(R^{2}=0.91\right)$. The trough levels $\left(C_{0}\right.$ or $\left.C_{24}\right)$ had a low correlation coefficient $\left(R^{2}\right.$ of 0.46 and 0.59 , respectively). A two-point LSS yielded both sampling times in the elimination phase $\left(C_{8}\right.$ and $\left.C_{12}\right)$. When a second sample point was added to a trough level $\left(C_{0}\right.$ or $\left.C_{24}\right)$, the $C_{8}$ sample was the best. In a three-point LSS, an early peak sample was added $\left(\mathrm{C}_{2}\right.$ or $\mathrm{C}_{3}$ in all cases. The time points as described by Niioka and SaintMarcoux were used in LRA and the results show that the correlation coefficient of both were numerically lower than the correlation coefficient of the single $C_{8}$ value. The regression and correlation coefficients of the different models and regression equations in the index population can be viewed in the Supplementary Table S4.1.

As for the predictive performance of various LSS by LRA, in the stable validation group 1, the single $C_{8}$-value predicted all AUC's correctly (Supplementary Table S4.2). Adding the $\mathrm{C}_{12}$ - and $\mathrm{C}_{2}$-values led to a numerical small improvement of accuracy and precision. When applying a single trough level $\left(C_{0}\right.$ or $\left.C_{24}\right)$, only $58 \%$ of the predicted 
AUC's were acceptable and there was a much stronger bias (MPE\%) compared with $\mathrm{C}_{8}$. This is also illustrated by the Bland Altman analysis in Figures $4.2 \mathrm{a}$ and $4.2 \mathrm{~b}$, showing a stronger bias especially for an $A \mathrm{CC}_{0-24}<200 \mu \mathrm{g} * \mathrm{hr} / \mathrm{l}$. Adding the $\mathrm{C}_{8}$ concentration to either the $C_{0}$ or the $C_{24}$ concentration, improved the predictive performance of such a LSS. Addition of a third concentration time point did not improve test characteristics.

In the de novo validation group 2 (day 14), measurement of a trough level as single time point (either $\mathrm{C}_{0}$ or $\mathrm{C}_{24}$ ) did not fulfil the pre-set requirements for prediction of AUC (Supplementary Table S4.3). Using $C_{8}$ as single time point, fulfilled the accuracy criteria in $75 \%$ of the cases, despite a bias (MPE\%) above the accepted $5 \%$ margin. Adding $\mathrm{C}_{12}$ to $\mathrm{C}_{8}$, improved the accuracy of this model, while the three-point strategy $\mathrm{C}_{8}-\mathrm{C}_{12}-\mathrm{C}_{2}$ performed best with correct prediction in all recipients. The improving agreement between the predicted and the measured $\mathrm{AUC}_{0-24}$ with this three-point LSS is also demonstrated in the Bland Altman plot in Figure $4.2 \mathrm{~d}-\mathrm{f}$. On day 42 similar results were derived (for this reason data not shown).

Forced entering of the time points described by Niioka (LRA) ${ }^{16}$ and Saint-Marcoux $(\mathrm{MAPB})^{14}$ (Supplementary Table S4.1) yielded comparable predictive performance in both validation groups (Supplementary Tables S4.2 and S4.3, respectively); the accuracy and precision were within the pre-set limits. Compared to our $\mathrm{C}_{8}$-model, they had a higher bias and numerically fewer patients fulfilled the criteria for accuracy, especially in the maintenance phase.

\section{Population pharmacokinetic modelling}

The results of the iterative two-stage Bayesian analysis and bootstrap analysis are displayed in the Supplementary Table S4.4. The bootstrap analysis confirmed the adequacy of the model. In validation group 1 (Supplementary Table S4.5), the predictive performance improved numerically with increasing number of samples. Adding a second sample in the elimination phase to an LSS that contains a forced trough level gives the biggest improvement in predictive performance, which is, however, still not better than single measurement of one sample in the elimination phase. This is similar to the results of validation of the LRA-based LSS's. Despite this numerical improvement with increasing number of included samples, contrary to the LSS's based on LRA, in the maintenance phase, all but the $C_{0}-C_{1}-C_{3}-L S S$ already met the pre-set criteria for bias, accuracy and precision. The $C_{0}-C_{1}-C_{3}$ LSS fell just outside the acceptance limits with a $-6,6 \%$ bias and only predicted 8 of the 12 AUC's with a MAPE of $<10 \%$. On an individual level we see that, as in the LRA-LSS, the trough level $\mathrm{C}_{0}$ only gave accurate prediction in 6 out of 12 cases. On the other hand, it is of notice that contrary to $\mathrm{C}_{0}$, using $\mathrm{C}_{24}$ in our MAPB-estimation already resulted in correct prediction in $90 \%$ of the cases. 

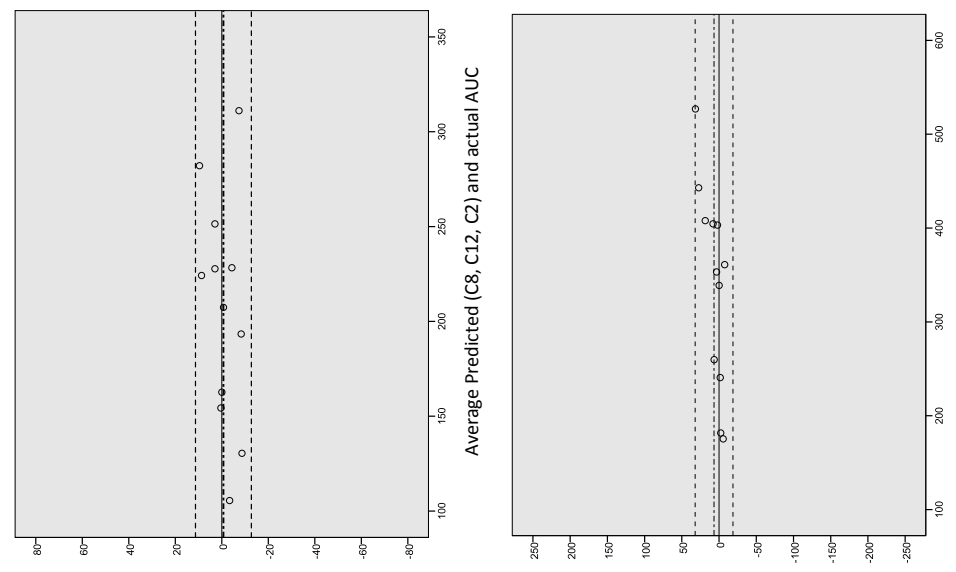

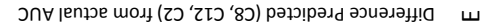

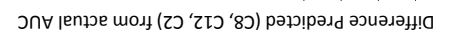

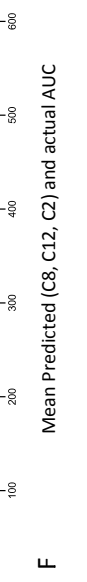

ปี่

我

늠

过 苗

을

ठ்

画
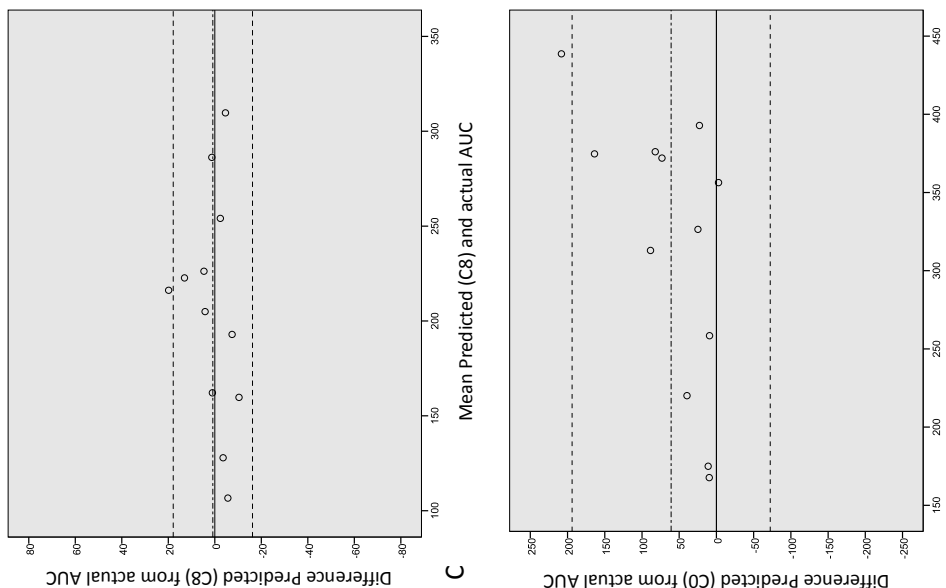

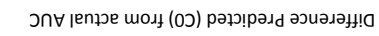

๑

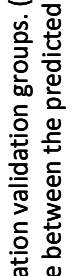
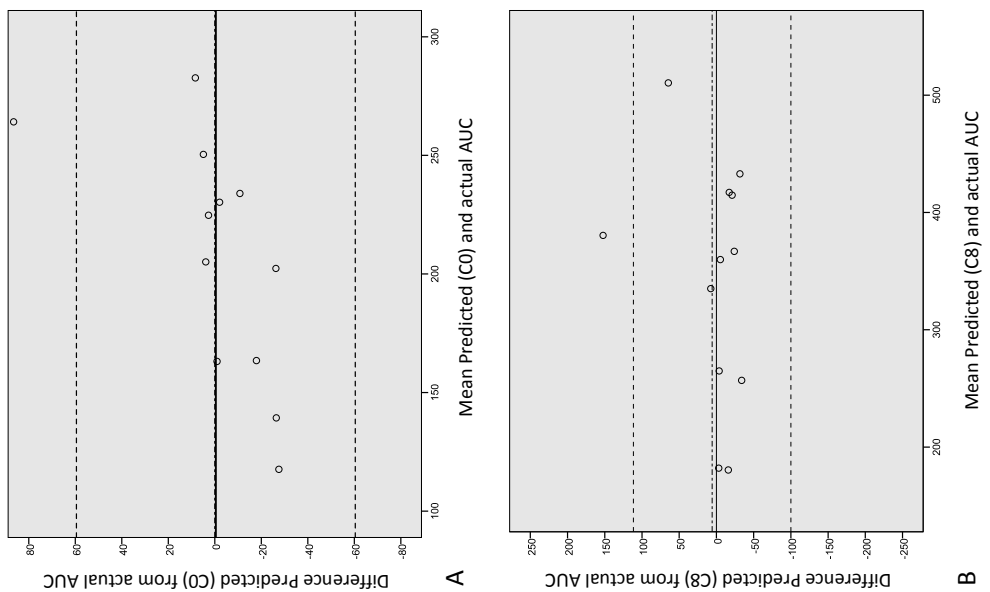

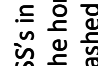

다을

o $\frac{1}{d}$

$\therefore$

¿

焉

듈

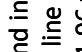

完岳卉

齐苋

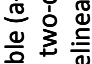

要

ป 웛

苍过

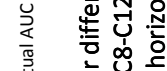

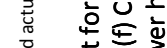

등 흥

응

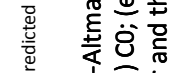

i

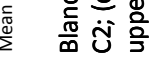

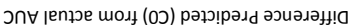

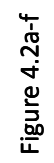


In validation group 2, a similar pattern is discernable (Supplementary Table S4.6). Here, as with LRA-LSS's, the predictive performance greatly benefits from the addition of a third sampling time near peak concentration with relative mean and root mean squared errors below $10 \%$. Comparable with the validation of the LRA-based LSS's, single time point measurement of a trough level does not meet predefined targets of accurate prediction of the $\mathrm{AUC}_{0-24}$ in the early post-transplant phase.

\section{D-optimal sampling times}

For the entire 24-hour period the D-optimal sampling times were $0.76,0.97,1.68$, 4.30 and 11.72 hours post-dose. The predictive performance of the combinations of 1 to 5 D-optimal sampling times is displayed in Supplementary Table S4.7. When choosing the optimal combination of 1 to 5 sampling times, the predictive performance increased from 1 to 5 samples with the largest added value of the second sample. In addition, we tested the predictive performance of a single pre-dose sample. This had a RMSE\% of $9.49 \%$ with a single sample and an MAPE\% of $9.01 \%$ and accurately (i.e. MAPE\% <10\%) predicted the $\mathrm{Cl} / \mathrm{F}$ in $76 \%$ of all cases (data not shown).

\section{Discussion}

This PK study in renal transplant recipients served to develop and validate a LSS for prediction of $\mathrm{AUC}_{0-24}$ for Tac $\mathrm{QD}$ based on LRA and compare its predictive performance with LSS's described in the literature and after application of optimal sampling times in a population PK model. For TDM purpose, it is of crucial importance to predict the exposure for patients with very low or very high exposure for prevention of graft loss and toxicity, respectively. Pharmacokinetic surrogates that correlate with these clinical outcome measures, e.g. $\mathrm{C}_{0}$, have evolved in the past decades. Recommendations define targets between 5 and $10 \mathrm{mcg} / \mathrm{l}$ as sufficient in a stable population for the twice daily tacrolimus formulation ${ }^{2}$ Our data show that the patients included in our model development set were within this range.

However, in renal transplant patients, a decrease in the apparent clearance (a measure for the AUC) of tacrolimus over time ${ }^{29,30}$ was not reflected in a corresponding change in $\mathrm{C}_{0}{ }^{31}$ Therapeutic drug monitoring by means of $\mathrm{C}_{0}$ measurement only, missed outlying drug exposure, as illustrated by the Bland Altman plot in Figure 4.2a. We observed in this PK study on LSS for estimation of $A \mathrm{CC}_{0-24}$ for Tac QD that a LSS based on LRA containing just the $\mathrm{C}_{8}$-value (i.e. a time point in the elimination phase) correlated highly with the $\mathrm{AUC}_{0-24}$ with a high predictive performance in stable renal transplant recipients.

In a study in Japanese renal transplant recipients it was concluded from linear regression analysis (LRA) that adding a concentration value at 12 hours after dosing 
$\left(C_{12}\right)$ to $C_{0}$ gave good prediction of the $A \cup C_{0-24}{ }^{16}$ Our findings agree with this model of Niioka et al. in that sampling during the elimination phase seems to be required for Tac QD to get an acceptable MAPE\% and RMSE\%. None of the strategies that emerged from our stepwise regression analysis contained a trough value. Niioka forced the $\mathrm{C}_{0}$ into their model, because they considered monitoring of tacrolimus using $\mathrm{C}_{12}$ alone to be uncommon. ${ }^{16}$ However, introduction of a trough level does not improve our model for recipients in the maintenance phase after kidney transplantation.

In the early post-transplant period (at day 14), we demonstrated that although a twosample LSS may have acceptable overall accuracy and precision, in a substantial number of cases the acceptance limits will be exceeded. In general, although sampling strategies may numerically predict drug exposure within the predefined acceptance criteria developed by Sheiner and Beal $^{27}$, a significant number of patients may have over- or underestimated drug exposure; an item that often is underreported. Therefore, in our early post-transplant population, a peak concentration $\left(C_{2}\right.$ or $\left.C_{3}\right)$ is required in addition to the values in the elimination phase $\left(C_{8}\right.$ and $\left.C_{12}\right)$, to reach individually acceptable accuracy and precision (Figure 4.2f). This is in accordance with the report of Saint Marcoux et al. They developed Bayesian estimators based on a 24-hour PK profile with combinations of a maximum of three sampling times within 4 hours post-dose (Maximum A Posteriori Bayesian (MAPB) analysis). ${ }^{13,14}$ In their first study, a model was developed and validated in 41 renal transplant recipients in the maintenance phase, while in a second study they applied MAPB in 12 kidney transplant patients at 2 and 6 weeks post-transplant. They report the optimal limited sampling schedule to consist of the time points pre-dose, 1 and 3 hours post-dose for both studies, i.e. also time points near the peak concentration. ${ }^{14}$

A major limitation of LSS on the basis of LRA is the need for exact timing of sampling. This can be avoided by using population kinetic parameter estimation. ${ }^{12}$ Therefore, we also developed a population kinetic model with first order absorption and first order elimination from the central compartment. In validation group 1 (stable), MAPBestimation resulted in excellent predictive performance in nearly all sampling strategies, with the exception of the $C_{0}$-level and to a lesser extent the $C_{0}-C_{1}-C_{3}$ LSS. In concert with the results of our LRA, addition of $C_{8}$ or $C_{12}$ greatly increases accuracy of LSS's. Using only the early post-dose sampling points apparently is insufficient for estimation of the AUC of an extended release drug. In contrast to single time point measurement of the $C_{0}$ trough level, the $C_{24}$ sampling time point gave better predictive accuracy and precision when implemented in MAPB-estimation. One may expect better correlation of the $C_{24}$ value with the $A_{U C} C_{0-24}$ since the $C_{24}$ value belongs to the same blood concentration profile that is to be predicted and does not contain the day-to-day intrapatient variability in drug exposure.

In validation group 2 (day 14), i.e. early post transplant, a similar pattern of improvements in predictive performance is discernable compared to the LRA-based LSS's. Addition of a third sampling time near peak plasma concentration further 
improves AUC estimation. In this group, only the raw concentration-time profiles were available without dosing and co-variable data. The individually calculated PK parameters will thus not reflect the true, and unknown, parameters. However, for evaluation of intrapatient predictive performance of specific sampling times the results are valid in our opinion.

The predictive performance of D-optimal sampling increased from 1 to 5 samples with the largest added value of the second sample, so that for LSS more frequent sampling has no added value. Our D-optimal times are spread around the absorption, distribution and elimination phases (sampling times at approximately 12 hours post dose) with adequate predictive performance, comparable to the LRA-based LSS's. This corroborates our findings of LRA, in which sampling strategies also covered the elimination phase.

Contrary to our results, Saint Marcoux et al. constrained sampling time combinations to the first 4 hours post-dose to find a good compromise between the precision of parameter estimates and possible implementation in clinical practice. ${ }^{14}$ Although not explicitly stated, we assume that in the paper of Benkali et al., the analyses were also constrained to the first hours post-dose, since this study is from the same group as in the Saint-Marcoux paper. ${ }^{13}$ However, our data showed that later sampling times in the elimination phase are the most relevant predictors of $A U C_{0-24}$ for Tac $Q D$, requiring easy and practical sampling for tacrolimus levels at these time points. In our institute, Dried Blood Spot analysis of tacrolimus has been developed and validated as being a reliable and practical method for home sampling at any desired time point. ${ }^{32,33}$ This method can easily be taught to patients and they find it convenient. Although this technique is not yet routinely applied, it can be easily implemented in centres were tacrolimus levels are measured by UPLC-MS/MS.

The strength of our study is that we disposed of a rich dataset comprised of three independent populations both early and late after renal transplantation with a sufficient number of 24-hour concentration-time profiles of each group. In addition, within these datasets we applied results from LRA in Bayesian forecasting also including later time points in the LSS's. As we did not have independent populations for both MAPB-model development and validation in the early post-transplant phase, we refrained from developing such a model in this group. Instead, we used the model developed in patients in the maintenance phase without the possibility to directly compare the Bayesian estimators as described by St Marcoux with a MAPB model in our population. Although we fulfilled the criteria set for the number of patients needed and the number was at least equal to previous studies, we consider that especially data on accuracy are based on a limited number of patients. Nevertheless, the similar results from our two different ways of analysis, LRA and MAPB, corroborated consistency of our conclusions.

In conclusion, we found that a LRA-based LSS containing one single measurement during the elimination phase $\left(C_{8}\right)$ gives accurate estimation of drug exposure in an 
adult white renal transplant population using Tac QD in the maintenance phase. This was confirmed in a population PK model (MAPB). None of the LSS's contained $\mathrm{C}_{0}$, the currently most used parameter for TDM of Tac QD, unless it was forced into a model in combination with other sampling time points. In the early post-transplant group, none of the single time-point LSS's adequately predicted $\mathrm{AUC}_{0-24} \mathrm{~A}$ model with the sampling triplet $C_{2}$, (a peak level) in combination with $C_{8}$ and $C_{12}$ (two samples in the elimination phase) gave the best prediction for the true $\mathrm{AUC}_{0-24}$ in the early posttransplant phase. Accurate prediction of drug exposure (i.e. by later and more frequent sampling) may outweigh convenience advantages of earlier sampling time points post-dose. The Dried Blood Spot method may facilitate this sampling in an outpatient setting. Whether adaptation of drug dosing based on more accurate measurement of drug exposure improves transplant outcome warrants further investigations.

\section{Compliance with ethical standards}

Ethical approval: All main studies cited above were conducted in accordance with the Declaration of Helsinki. The protocols had been reviewed by the Ethics Committee at each study center and each patient had given written informed consent prior to enrollment into the study. The main studies were conducted between 2003 and $2004^{19,20}$, i.e. before the cut-off date for registration of new and ongoing clinical trials and, thus, were not registered in a public trials registry. Collection, storage, and use of patient data were performed in agreement with FEDERA (Federation of Dutch University Medical Centers) Code of Conduct (www.nfu.nl). 


\section{References}

1. Staatz CE, Tett SE. Clinical Pharmacokinetics of Once-Daily Tacrolimus in Solid-Organ Transplant Patients. Clin Pharmacokinet. 2015;54(10):993-1025.

2. Wallemacq P, Armstrong VW, Brunet M, Haufroid V, Holt DW, Johnston A, et al. Opportunities to optimize tacrolimus therapy in solid organ transplantation: report of the European consensus conference. Therapeutic drug monitoring. 2009;31(2):139-152.

3. Mahalati K, Kahan BD. Pharmacological surrogates of allograft outcome. Ann Transplant. 2000;5(2):14-23.

4. Undre NA, van Hooff J, Christiaans M, Vanrenterghem Y, Donck J, Heeman U, et al. Low systemic exposure to tacrolimus correlates with acute rejection. Transplant Proc. 1999;31(1-2):296-298.

5. Pisitkun T, Eiam-Ong S, Chusil S, Praditpornsilpa K, Pansin P, Tungsanga K. The roles of C4 and AUCO-4 in monitoring of tacrolimus in stable kidney transplant patients. Transplant Proc. 2002;34(8): 3173-3175.

6. Stolk LM, Van Duijnhoven EM, Christiaans MH, van Hooff JP. Trough levels of tacrolimus. Therapeutic drug monitoring. 2002;24(4):573; author reply 573-574.

7. Wong KM, Shek CC, Chau KF, Li CS. Abbreviated tacrolimus area-under-the-curve monitoring for renal transplant recipients. Am J Kidney Dis. 2000;35(4):660-666.

8. Op den Buijsch RA, van de Plas A, Stolk LM, Christiaans MH, van Hooff JP, Undre NA, et al. Evaluation of limited sampling strategies for tacrolimus. Eur J Clin Pharmacol. 2007;63(11):1039-1044.

9. Alloway R, Steinberg S, Khalil K, Gourishankar S, Miller J, Norman D, et al. Conversion of stable kidney transplant recipients from a twice daily Prograf-based regimen to a once daily modified release tacrolimus-based regimen. Transplant Proc. 2005;37(2):867-870.

10. Advagraf: EPAR - Scientific Discussion: European Medicines Agency; 2007.

11. Florman S, Alloway R, Kalayoglu M, Lake K, Bak T, Klein A, et al. Conversion of stable liver transplant recipients from a twice-daily Prograf-based regimen to a once-daily modified release tacrolimusbased regimen. Transplant Proc. 2005;37(2):1211-1213.

12. Marquet P, Bedu A, Monchaud C, Saint-Marcoux F, Rerolle JP, Etienne I, et al. Pharmacokinetic Therapeutic Drug Monitoring of Advagraf in More Than 500 Adult Renal Transplant Patients, Using an Expert System Online. Ther Drug Monit. 2018;40(3):285-291.

13. Benkali K, Rostaing L, Premaud A, Woillard JB, Saint-Marcoux F, Urien S, et al. Population pharmacokinetics and Bayesian estimation of tacrolimus exposure in renal transplant recipients on a new once-daily formulation. Clin Pharmacokinet. 2010;49(10):683-692.

14. Saint-Marcoux F, Debord J, Undre N, Rousseau A, Marquet P. Pharmacokinetic modeling and development of Bayesian estimators in kidney transplant patients receiving the tacrolimus once-daily formulation. Ther Drug Monit. 2010;32(2):129-135.

15. Woillard JB, de Winter BC, Kamar N, Marquet P, Rostaing L, Rousseau A. Population pharmacokinetic model and Bayesian estimator for two tacrolimus formulations--twice daily Prograf and once daily Advagraf. Br J Clin Pharmacol. 2011;71(3):391-402.

16. Niioka T, Miura M, Kagaya $H$, Saito M, Numakura K, Habuchi T, et al. A limited sampling strategy to estimate the area under the concentration-time curve of tacrolimus modified-release once-daily preparation in renal transplant recipients. Ther Drug Monit. 2013;35(2):228-232.

17. van Hooff JP, Alloway RR, Trunecka P, Mourad M. Four-year experience with tacrolimus once-daily prolonged release in patients from phase II conversion and de novo kidney, liver, and heart studies. Clin Transplant. 2011;25(1):E1-12.

18. Stifft F, Undre N, JP VH, Christiaans MH. Effect of breakfast on the exposure of the once-daily Tacrolimus formulation in stable kidney transplant recipients. Ther Drug Monit. 2016;38(4):456-462.

19. van Hooff J, Van der Walt I, Kallmeyer J, Miller D, Dawood S, Moosa MR, et al. Pharmacokinetics in stable kidney transplant recipients after conversion from twice-daily to once-daily tacrolimus formulations. Ther Drug Monit. 2012 Feb;34(1):46-52. 
20. Wlodarczyk Z, Squifflet JP, Ostrowski M, Rigotti P, Stefoni S, Citterio F, et al. Pharmacokinetics for once- versus twice-daily tacrolimus formulations in de novo kidney transplantation: a randomized, open-label trial. Am J Transplant. 2009;9(11):2505-2513.

21. Touw DJ, Vinks AA, Neef C. Pharmacokinetic modelling of intravenous tobramycin in adolescent and adult patients with cystic fibrosis using the nonparametric expectation maximization (NPEM) algorithm. Pharm World Sci. 1997;19(3):142-151.

22. Note for Guidance on the Investigation of Bioavailability and Bioequivalence (NfG on BA BE). London: European Medicines Agency; 2010.

23. Fuchs A, Csajka C, Thoma Y, Buclin T, Widmer N. Benchmarking therapeutic drug monitoring software: a review of available computer tools. Clin Pharmacokinet. 2013;52(1):9-22.

24. Proost JH, Eleveld DJ. Performance of an iterative two-stage bayesian technique for population pharmacokinetic analysis of rich data sets. Pharm Res. 2006;23(12):2748-2759.

25. Xue L, Zhang H, Ma S, Rui JZ, Miao LY. Population pharmacokinetics and pharmacogenetics of tacrolimus in healthy Chinese volunteers. Pharmacology. 2011;88(5-6):288-294.

26. D'Argenio DZ. Optimal sampling times for pharmacokinetic experiments. J Pharmacokinet Biopharm. 1981;9(6):739-756.

27. Sheiner LB, Beal SL. Some suggestions for measuring predictive performance. J Pharmacokinet Biopharm. 1981;9(4):503-512.

28. Bland JM, Altman DG. Statistical methods for assessing agreement between two methods of clinical measurement. Lancet. 1986;1(8476):307-310.

29. de Jonge $\mathrm{H}$, Vanhove $\mathrm{T}$, de Loor $\mathrm{H}$, Verbeke $\mathrm{K}$, Kuypers DR. Progressive decline in tacrolimus clearance after renal transplantation is partially explained by decreasing CYP3A4 activity and increasing haematocrit. Br J Clin Pharmacol. 2015;80(3):548-559.

30. Stifft F, van Kuijk SMJ, Bekers O, Christiaans MHL. Increase in tacrolimus exposure after steroid tapering is influenced by CYP3A5 and pregnane $\mathrm{X}$ receptor genetic polymorphisms in renal transplant recipients. Nephrology, dialysis, transplantation : official publication of the European Dialysis and Transplant Association - European Renal Association. 2018;33(9):1668-1675.

31. Scholten EM, Cremers SC, Schoemaker RC, Rowshani AT, van Kan EJ, den Hartigh J, et al. AUC-guided dosing of tacrolimus prevents progressive systemic overexposure in renal transplant recipients. Kidney Int. 2005;67(6):2440-2447.

32. Hoogtanders K, van der Heijden J, Christiaans M, Edelbroek P, van Hooff JP, Stolk LM. Therapeutic drug monitoring of tacrolimus with the dried blood spot method. J Pharm Biomed Anal. 2007;44(3): 658-664.

33. Hoogtanders K, van der Heijden J, Christiaans M, van de Plas A, van Hooff J, Stolk L. Dried blood spot measurement of tacrolimus is promising for patient monitoring. Transplantation. 2007;83(2):237-238. 


\section{Supplement: Appendix A}

\section{D-optimality and Maximum A Posteriori Bayesian estimation}

\section{D-optimality}

D-optimality uses Fisher information ${ }^{1}$, with calculation of the Fisher information matrix (FIM), which is calculated as:

$$
F I M=J^{T} R^{-1} J
$$

Where $P$ is the Jacobian matrix, $P^{\top}$ the transpose of the Jacobian matrix and $R^{-1}$ is the inverse of the variance matrix, signifying the weights attached to the measurements. The Jacobian matrix is defined as the matrix containing all first order derivatives of a vector valued function. Let $C\left(P^{*}, t_{m}\right)$ the concentration of drug in a PK compartment as a function of parameter $\mathrm{P}^{*}$ at time $\mathrm{t}$. Then, the Jacobian matrix is of the following NxM form:

$$
J=\left[\begin{array}{cccc}
\frac{d C\left(P^{*}, t_{1}\right)}{d P_{1}} & \bullet & \bullet & \frac{d C\left(P^{*}, t_{1}\right)}{d P_{m}} \\
\bullet & \bullet & \bullet \\
\bullet & \bullet & \bullet \\
\frac{d C\left(P^{*}, t_{n}\right)}{d P_{1}} & \bullet & \bullet & \frac{d C\left(P^{*}, t_{n}\right)}{d P_{m}}
\end{array}\right]
$$

Equation (2)

And Matrix $R$ is defined as:

$R=\left[\begin{array}{cccc}S D_{1}^{2} & & & 0 \\ & \bullet & & \\ & & \bullet & \\ 0 & & & S D_{i}^{2}\end{array}\right]$

Equation (3)

Where SDi is the standard deviation of measurement $\mathrm{i}$.

The determinant of the FIM is then calculated. The maximum of the determinant of the FIM over the dosing interval is calculated using an adaptation of the guided Monte Carlo search algorithm previously described. ${ }^{2}$ 


\section{Maximum A Posteriori Bayesian estimation}

Concentrations are calculated using integrated equations, similar to the ones used in MW \Pharm 3.80. Individual MAPB estimates are calculated by minimizing the following objective function:

$$
\sum_{i=1}^{n} \frac{\left(C_{i, o b s}-C_{i, p t}\right)^{2}}{S D^{2} C_{i, o b s}}+\sum_{j=1}^{m} \frac{\left(P_{j, p o p}-P_{j, p t}\right)^{2}}{S D^{2} P_{j, p o p}}
$$

Where $n$ is the number of observed plasma concentrations, $m$ is the number of parameters, $P_{p o p}$ and $P_{p t}$ are the log transformed parameter estimates of the population model and the individual estimates, respectively; $\mathrm{C}_{\mathrm{obs}}$ represents the observed plasma concentrations and $C_{p t}$ represents the patients' predicted concentration; $S D P_{\text {pop }}$ represents the standard deviation of the log transformed population PK parameter and SD $C_{o b s}$ represents the standard deviation of the observed plasma concentrations, or the residual error. Minimization of the objective function is performed by an adaptation of the Nelder-Mead simplex algorithm. ${ }^{3}$ The above calculations are made using custom built software based on the VB.net programming language implemented in Microsoft Visual Studio 2008.

\section{References for Appendix A}

1 D'Argenio DZ. Optimal sampling times for pharmacokinetic experiments. J Pharmacokinet Biopharm. 1981;9(6):739-756.

2 Delgoda R, Pulfer JD. A Guided Monte Carlo Search Algorithm for Global Optimization of Multidimensional Functions. Journal of chemical information and computer sciences 1998;38(6): 1087-1095.

3 Nelder JA, Mead K. A simplex method for function minimization. The Computer Journal 1965;7(4): 308-313. 
Table S4.1 Results from linear regression analysis with correlation and regression equations of different time point LSS with $\mathrm{AUC}_{0-24}$ hours (Index group, $\mathrm{n}=27$ ).

\begin{tabular}{lccl}
\hline Parameter & $\mathrm{R}$ & $\mathrm{R}^{2}$ & Equation \\
\hline $\mathrm{C}_{8}{ }^{a}$ & 0.95 & 0.903 & $8.238+22.112^{*} \mathrm{C} 8$ \\
$\mathrm{C}_{8}, \mathrm{C}_{12}{ }^{\mathrm{a}}$ & 0.971 & 0.942 & $12.997+12.561^{*} \mathrm{C} 8+10.148^{*} \mathrm{C} 12$ \\
$\mathrm{C}_{8}, \mathrm{C}_{12}, \mathrm{C}_{2}{ }^{\mathrm{a}}$ & 0.989 & 0.979 & $10.786+8.961^{*} \mathrm{C} 8+10.011^{*} \mathrm{C} 12+2.506^{*} \mathrm{C} 2$ \\
& & & \\
$\mathrm{C}_{0}{ }^{\mathrm{b}}$ & 0.677 & 0.458 & $64.757+22.556 * \mathrm{C} 0$ \\
$\mathrm{C}_{0}, \mathrm{C}_{8}{ }^{\mathrm{b}, \mathrm{a}}$ & 0.953 & 0.908 & $1.771+3.062^{*} \mathrm{C} 0+20.708^{*} \mathrm{C} 8$ \\
$\mathrm{C}_{0}, \mathrm{C}_{8}, \mathrm{C}_{2}{ }^{\mathrm{b}, \mathrm{a}}$ & 0.974 & 0.950 & $-2.482+3.996^{*} \mathrm{C} 0+16.317^{*} \mathrm{C} 8+2.662^{*} \mathrm{C} 2$ \\
$\mathrm{C}_{24}{ }^{\mathrm{b}}$ & & & \\
$\mathrm{C}_{24}, \mathrm{C}_{8}{ }^{\mathrm{b}, \mathrm{a}}$ & 0.768 & 0.589 & $22.992+29.426^{*} \mathrm{C} 24$ \\
$\mathrm{C}_{24}, \mathrm{C}_{8}, \mathrm{C}_{3}{ }^{\mathrm{b}, \mathrm{a}}$ & 0.959 & 0.920 & $-8.047+7.018^{*} \mathrm{C} 24+19.076^{*} \mathrm{C} 8$ \\
$\mathrm{C}_{0}, \mathrm{C}_{12}{ }^{\mathrm{c}}$ & 0.983 & 0.967 & $-23.056+10.432^{*} \mathrm{C} 24+12.416^{*} \mathrm{C} 8+4.281^{*} \mathrm{C} 3$ \\
$\mathrm{C}_{0}, \mathrm{C}_{1}, \mathrm{C}_{3}{ }^{\mathrm{d}}$ & & & \\
\hline
\end{tabular}

$C$ with subscript denotes the tacrolimus concentration measured at that specific time point. Linear Regression Methods: $a=$ method stepwise; $b=$ forced enter $C_{0}$ or $C_{24}$ and stepwise method; $c=$ forced enter of time points of Niioka study ${ }^{20} ; d=$ forced enter of time points of Saint-Marcoux study. ${ }^{1}$

Table S4.2 Predictive performance of LSS's by linear regression analysis in validation group $1(n=12)$.

\begin{tabular}{lccccc}
\hline Model & $\begin{array}{c}\text { Bias } \\
\text { MPE(\%) (SD) }\end{array}$ & $\begin{array}{c}\text { Accuracy } \\
\text { MAPE(\%) (SD) }\end{array}$ & $\begin{array}{c}\text { Precision } \\
\text { RMSE (\%) }\end{array}$ & $\mathrm{R}^{2}$ & $\mathrm{~N}$ MAPE <10\% \\
\hline $\mathrm{C}_{8}$ & $0.14(4.46)$ & $3.41(2.69)$ & 4.28 & 0.990 & $12 / 12(100 \%)$ \\
$\mathrm{C}_{8}, \mathrm{C}_{12}$ & $0.60(3.29)$ & $2.57(2.00)$ & 3.20 & 0.995 & $12 / 12(100 \%)$ \\
$\mathrm{C}_{8}, \mathrm{C}_{12}, \mathrm{C}_{2}$ & $0.78(3.14)$ & $2.42(2.03)$ & 3.10 & 0.995 & $12 / 12(100 \%)$ \\
& & & & & \\
$\mathrm{C}_{0}$ & $3.59(13.98)$ & $9.60(10.43)$ & 13.85 & 0.891 & $7 / 12(58 \%)$ \\
$\mathrm{C}_{0}, \mathrm{C}_{8}$ & $0.10(3.22)$ & $2.53(1.84)$ & 3.08 & 0.993 & $12 / 12(100 \%)$ \\
$\mathrm{C}_{0}, \mathrm{C}_{8}, \mathrm{C}_{2}$ & $-0.03(3.74)$ & $2.80(2.34)$ & 3.58 & 0.992 & $12 / 12(100 \%)$ \\
& & & & & \\
$\mathrm{C}_{24}$ & $7.26(9.46)$ & $9.25(7.31)$ & 11.60 & 0.948 & $7 / 12(58 \%)$ \\
$\mathrm{C}_{24}, \mathrm{C}_{8}$ & $1.40(5.37)$ & $4.97(1.82)$ & 4.63 & 0.990 & $12 / 12(100 \%)$ \\
$\mathrm{C}_{24}, \mathrm{C}_{8}, \mathrm{C}_{3}$ & $0.65(6.23)$ & $4.96(3.42)$ & 5.31 & 0.998 & $11 / 12(92 \%)$ \\
$\mathrm{C}_{0}, \mathrm{C}_{12}$ & & & & & \\
$\mathrm{C}_{0}, \mathrm{C}_{1}, \mathrm{C}_{3}$ & $1.62(6.59)$ & $4.97(4.40)$ & 6.51 & 0.969 & $10 / 12(83 \%)$ \\
\hline $\mathrm{C}_{w}$ & $1.26(6.27)$ & $4.55(4.3)$ & 6.14 & 0.982 & $9 / 12(75 \%)$ \\
\hline
\end{tabular}

$C$ with subscript denotes the tacrolimus concentration measured at that specific time point.

$\mathrm{MPE}=$ Mean Prediction Error; MAPE = Mean Absolute Prediction Error; RMSE = Root Mean Squared Error; $\mathrm{N} M A P E<10 \%=$ number of patients $(\%)$ with a MAPE\% of less than $10 \%$. 
Table S4.3 Predictive performance of LSS's by linear regression analysis in validation group 2 ( $n=12$ ).

\begin{tabular}{lccccc}
\hline Model & $\begin{array}{c}\text { Bias } \\
\text { MPE(\%) (SD) }\end{array}$ & $\begin{array}{c}\text { Accuracy } \\
\text { MAPE(\%) (SD) }\end{array}$ & $\begin{array}{c}\text { Precision } \\
\text { RMSE (\%) }\end{array}$ & $\mathrm{R}^{2}$ & N MAPE\% $<10 \%$ \\
\hline $\mathrm{C}_{8}$ & $0.35(12.42)$ & $8.26(8.94)$ & 11.9 & 0.883 & $9 / 12(75 \%)$ \\
$\mathrm{C}_{8}, \mathrm{C}_{12}$ & $-0.35(8.11)$ & $5.67(5.55)$ & 7.78 & 0.956 & $10 / 12(83 \%)$ \\
$\mathrm{C}_{8}, \mathrm{C}_{12}, \mathrm{C}_{2}$ & $-1.25(3.01)$ & $2.46(2.04)$ & 3.14 & 0.997 & $12 / 12(100 \%)$ \\
& & & & \\
$\mathrm{C}_{0}$ & $-15.04(12.81)$ & $15.18(12.62)$ & 19.41 & 0.824 & $6 / 12(50 \%)$ \\
$\mathrm{C}_{0}, \mathrm{C}_{8}$ & $0.69(12.07)$ & $8.08(8.66)$ & 11.58 & 0.885 & $9 / 12(75 \%)$ \\
$\mathrm{C}_{0}, \mathrm{C}_{8}, \mathrm{C}_{2}$ & $-0.17(6.51)$ & $5.09(3.76)$ & 6.24 & 0.971 & $11 / 12(92 \%)$ \\
& & & & & \\
$\mathrm{C}_{24}$ & $-12.73(12.11)$ & $13.75(10.83)$ & 17.22 & 0.886 & $5 / 12(42 \%)$ \\
$\mathrm{C}_{24}, \mathrm{C}_{8}$ & $0.64(11.47)$ & $8.36(7.46)$ & 11.00 & 0.898 & $9 / 12(75 \%)$ \\
$\mathrm{C}_{24}, \mathrm{C}_{8}, \mathrm{C}_{3}$ & $4.29(6.65)$ & $6.65(4.01)$ & 7.68 & 0.979 & $10 / 12(83 \%)$ \\
$\mathrm{C}_{0}, \mathrm{C}_{12}$ & & & & \\
$\mathrm{C}_{0}, \mathrm{C}_{1}, \mathrm{C}_{3}$ & $-3.71(8.25)$ & $7.53(4.60)$ & 8.73 & 0.957 & $10 / 12(83 \%)$ \\
\hline
\end{tabular}

$C$ with subscript denotes the tacrolimus concentration measured at that specific time point.

$\mathrm{MPE}=$ Mean Prediction Error; MAPE = Mean Absolute Prediction Error; RMSE = Root Mean Squared Error; $\mathrm{N}$ MAPE $<10 \%$ = number of patients (\%) with a MAPE\% of less than $10 \%$.

Table S4.4 Population pharmacokinetic model.

\begin{tabular}{lcccccc}
\hline & \multicolumn{3}{c}{ Base model } & \multicolumn{3}{c}{ Bootstrap analysis } \\
Parameters & Mean & \multicolumn{1}{c}{ SD } & CV(\%) & Median & Mean 95\% Cl & SD 95\% Cl \\
\hline Cl/F (I/hr) & 17.176 & 9.945 & 57.9 & 17.163 & $13.930-21.061$ & $5.575-13.977$ \\
V1/F (I/kg) & 0.362 & 0.201 & 55.4 & 0.334 & $0.221-0.521$ & $0.101-0.277$ \\
Cl12/F (I/hr) & 90.419 & 49.525 & 54.7 & 89.525 & $67.782-121.928$ & $26.759-74.005$ \\
V2/F (I/kg) & 11.445 & 4.909 & 42.9 & 11.435 & $9.082-16.550$ & $2.337-7.374$ \\
Ka (/hr) & 0.335 & 0.119 & 35.6 & 0.330 & $0.253-0.464$ & $0.032-0.196$ \\
Lag time & 0.760 & 0 & 0 & 0.776 & $0.672-0.836$ & $0-0$ \\
\hline
\end{tabular}

$\mathrm{SD}=$ Standard deviatio; $\mathrm{CV}(\%)=$ Variation coefficient in \%; $\mathrm{Cl}=$ Confidential Interval; $\mathrm{Cl} / \mathrm{F}=\mathrm{Apparent}$ total clearance from plasma; $\mathrm{V} 1 / \mathrm{F}=\mathrm{Apparent}$ volume of distribution of the central compartemt; $\mathrm{V} 2 / \mathrm{F}=\mathrm{Apparent}$ volume of distribution of the peripheral compartment; $\mathrm{CL}$ 12/F Apparent clearance from the central and peripheral compartment; $\mathrm{Ka}=$ Absorption rate constant. 
Table S4.5 Predictive performance of LSS's by Maximum A Posteriori Bayesian estimation in validation group 1.

\begin{tabular}{lccccc}
\hline Model & $\begin{array}{c}\text { Bias } \\
\text { MPE\% }\end{array}$ & $\begin{array}{c}\text { Accuracy } \\
\text { MAPE\% }\end{array}$ & $\begin{array}{c}\text { Precision } \\
\text { RMSE\% }\end{array}$ & $\mathrm{R}^{2}$ & $\mathrm{~N} \mathrm{MAPE \% <10 \%}$ \\
\hline $\mathrm{C}_{8}$ & +5.0 & 6.9 & 8.9 & 0.948 & $10 / 12(83.3)$ \\
$\mathrm{C}_{8}, \mathrm{C}_{12}$ & +3.0 & 4.4 & 5.6 & 0.978 & $10 / 12(83.3)$ \\
$\mathrm{C}_{8}, \mathrm{C}_{12}, \mathrm{C}_{2}$ & -1.1 & 3.2 & 3.9 & 0.979 & $12 / 12(100)$ \\
& & & & & \\
$\mathrm{C}_{0}$ & -4.2 & 8.2 & 9.3 & 0.933 & $6 / 12(50.0)$ \\
$\mathrm{C}_{0}, \mathrm{C}_{8}$ & +1.2 & 4.3 & 5.9 & 0.965 & $10 / 12(83.3)$ \\
$\mathrm{C}_{0}, \mathrm{C}_{8}, \mathrm{C}_{2}$ & -0.7 & 4.2 & 5.0 & 0.975 & $12 / 12(100)$ \\
$\mathrm{C}_{24}$ & & & & & \\
$\mathrm{C}_{24}, \mathrm{C}_{8}$ & +1.0 & 4.5 & 5.6 & 0.964 & $11 / 12(91.7)$ \\
$\mathrm{C}_{24}, \mathrm{C}_{8}, \mathrm{C}_{3}$ & +3.3 & 4.4 & 5.9 & 0.980 & $10 / 12(83.3)$ \\
$\mathrm{C}_{0}, \mathrm{C}_{12}$ & +1.0 & 2.3 & 2.9 & 0.994 & $12 / 12(100)$ \\
$\mathrm{C}_{0}, \mathrm{C}_{1}, \mathrm{C}_{3}$ & & & & & \\
\hline
\end{tabular}

$C$ with subscript denotes the tacrolimus concentration measured at that specific time point.

$\mathrm{MPE}=$ Mean Prediction Error; MAPE = Mean Absolute Prediction Error; RMSE = Root Mean Squared Error; N MAPE $<10 \%$ = number of patients (\%) with a MAPE\% of less than $10 \%$.

Table S4.6 Predictive performance of LSS by Maximum A Posteriori Bayesian estimation in validation group 2.

\begin{tabular}{lccccr}
\hline Model & $\begin{array}{c}\text { Bias } \\
\text { MPE\% }\end{array}$ & $\begin{array}{c}\text { Accuracy } \\
\text { MAPE\% }\end{array}$ & $\begin{array}{c}\text { Precision } \\
\text { RMSE\% }\end{array}$ & $\mathrm{R}^{2}$ & $\mathrm{~N} \mathrm{MAPE \% <10 \%}$ \\
\hline $\mathrm{C}_{8}$ & +1.8 & 8.9 & 12.1 & 0.778 & $9 / 12(75.0)$ \\
$\mathrm{C}_{8}, \mathrm{C}_{12}$ & +1.6 & 8.7 & 10.0 & 0.856 & $9 / 12(75.0)$ \\
$\mathrm{C}_{8}, \mathrm{C}_{12}, \mathrm{C}_{2}$ & -1.0 & 5.3 & 7.0 & 0.958 & $10 / 12(83.3)$ \\
& & & & & \\
$\mathrm{C}_{0}$ & -6.5 & 11.3 & 14.8 & 0.679 & $7 / 12(58.3)$ \\
$\mathrm{C}_{0}, \mathrm{C}_{8}$ & -2.0 & 7.3 & 11.4 & 0.777 & $9 / 12(75.0)$ \\
$\mathrm{C}_{0}, \mathrm{C}_{8}, \mathrm{C}_{2}$ & -2.9 & 4.2 & 6.2 & 0.950 & $11 / 12(91.7)$ \\
$\mathrm{C}_{24}$ & & & & & \\
$\mathrm{C}_{24}, \mathrm{C}_{8}$ & -9.6 & 10.9 & 14.2 & 0.784 & $7 / 12(58.3)$ \\
$\mathrm{C}_{24}, \mathrm{C}_{8}, \mathrm{C}_{3}$ & -3.4 & 6.5 & 10.5 & 0.820 & $10 / 12(83.3)$ \\
$\mathrm{C}_{0}, \mathrm{C}_{12}$ & -1.3 & 4.6 & 5.6 & 0.959 & $10 / 12(83.3)$ \\
$\mathrm{C}_{0}, \mathrm{C}_{1}, \mathrm{C}_{3}$ & & & & & \\
\hline
\end{tabular}

$C$ with subscript denotes the tacrolimus concentration measured at that specific time point.

$\mathrm{MPE}=$ Mean Prediction Error; MAPE = Mean Absolute Prediction Error; RMSE = Root Mean Squared Error; $\mathrm{N} M A P E<10 \%=$ number of patients (\%) with a MAPE\% of less than $10 \%$. 
Table S4.7 Predictive performance of Bayesian LSS's.

\begin{tabular}{lccccc}
\hline $\begin{array}{l}\text { Sampling times } \\
\text { (hours post dose) }\end{array}$ & $\begin{array}{c}\text { Bias } \\
\text { MPE(\%) }\end{array}$ & $\begin{array}{c}\text { Accuracy } \\
\text { MAPE(\%) }\end{array}$ & $\begin{array}{c}\text { Precision } \\
\text { RMSE (\%) }\end{array}$ & $\mathrm{R}^{2}$ & N MAPE $<10 \%$ \\
\hline 11.72 & 0.10 & 6.23 & 8.65 & 0.945 & $82 \%$ \\
$1.67,11.72$ & 0.40 & 2.44 & 3.45 & 0.992 & $98 \%$ \\
$1.67,4.30,11.72$ & 0.08 & 1.77 & 2.37 & 0.997 & $99 \%$ \\
$0.76,1.67,4.30,11.72$ & 0.06 & 1.02 & 1.48 & 0.998 & $100 \%$ \\
$0.76,0.97,1.67,4.30,11.72$ & 0.01 & 1.03 & 1.53 & 0.997 & $100 \%$ \\
\hline
\end{tabular}

$\mathrm{MPE}=$ Mean Prediction Error; MAPE = Mean Absolute Prediction Error; RMSE $=$ Root Mean Squared Error; N MAPE $<10 \%$ = percentage of simulated patients with a MAPE\% of less than $10 \%$ 



\title{
Chapter 5
}

Effect of breakfast on the exposure of the once-daily Tacrolimus formulation in stable kidney transplant recipients

\author{
F. Stifft, N. Undre, J. van Hooff, M. Christiaans
}

Therapeutic drug monitoring 2016;38(4):456-462 


\begin{abstract}

\section{Background}

The once-daily Tacrolimus formulation (Tac QD) has to be taken on an empty stomach. This is inconvenient for patients and may hamper compliance. The influence of food intake on the exposure of Tac QD is unknown in transplant recipients. We compared the pharmacokinetics (PK) of Tac QD in stable kidney transplant recipients under fasted and fed conditions.
\end{abstract}

\title{
Methods
}

In an open-label, single centre cross-over PK study, 27 stable kidney white transplant recipients (17 male, 10 female) treated with Tac QD under fasted conditions were enrolled. Two 10-point 24-hour blood concentration-time profiles $\left(\mathrm{AUC}_{0-24}\right)$ were collected under steady state conditions. The primary objective was to investigate the effect of food on the pharmacokinetics and relative bioavailability of Tac QD.

\section{Results}

27 stable renal transplant patients provided one $\mathrm{AUC}_{0-24}$ under fasted and fed conditions, respectively. $A \cup C_{0-24}, C_{24}$ and $C_{\max }$, were lower in the fed state and $T_{\max }$ was 1 hour postponed. The $90 \%$ confidence interval ratio (fed: fasted) for $A \mathrm{CC}_{0-24}$ was $0.81-0.91$ and for $\mathrm{C}_{24} 0.82-0.92$ (both $\mathrm{p}<0.001$ ). The majority $(60 \%)$ had no significant change, but the change in $\mathrm{AUC}_{0-24}$ ranged from $-38 \%$ to $+29 \%$. One trough level was below the target range after fed intake.

\section{Conclusions}

When Tac QD is ingested with standard continental breakfast, $\mathrm{AUC}_{0-24}$ and $\mathrm{C}_{24}$ decrease overall, with $\mathrm{C}_{24}$ in the therapeutic range in almost all patients. The convenient fed intake could promote therapy adherence. Given the possible significant change in exposure, we advise monitoring of the tacrolimus trough level one week after fed ingestion of Tac QD 


\section{Introduction}

Tacrolimus (Tac) is the cornerstone immunosuppressive drug treatment in solid organ transplantation and has been available as an immediate release formulation (Tac BID, Prograf) administered twice daily since 1993. The effect of food on the exposure of Tac when administered as the BID formulation has been investigated before. ${ }^{1-3}$ The presence of food decreased both the rate and the extent of absorption of Tac, with the effect being most pronounced after a high-fat meal. ${ }^{1}$ This observation led to the recommendation that Tac should be administered on an empty stomach, that is, one hour before or two hours after a meal.

However, this is not convenient for patients and may lead to decrease in compliance. Therefore, in previous studies our group investigated pharmacokinetic parameters of Tac BID after ingestion of the Tac in a fasting state vs. a fed state, that is, together with a continental breakfast. ${ }^{2,4}$ These studies showed, that conversion to fed ingestion of Tac, without dose adjustments, did not significantly change Tac trough levels in stable renal transplant recipients. ${ }^{4}$ In addition, the differences in bioavailability (AUC) were not significantly reduced, but variable between patients, with the smallest difference three weeks after transplantation. ${ }^{2}$ Hence in our centre, patients are converted to fed ingestion of Tac BID in this early post transplantation phase, provided Tac trough levels are within the therapeutic range.

A once-daily formulation (Tac QD, Advagraf ${ }^{\circ}$ ) has become available for several years by now. The efficacy and safety profile of Tac QD is comparable to that of Tac BID in de novo and in stable kidney transplant recipients after conversion. ${ }^{5-7}$ Based on studies in healthy volunteers, reduced bioavailability with fed intake led to the advice to ingest Tac QD on an empty stomach. ${ }^{8}$ To our knowledge the influence of food intake on the bioavailability of this newer formulation has not formerly been reported yet in kidney transplant recipients. Establishing the effect of meals on absorption could help practitioners with the management of their patients on a daily basis. We therefore compared the exposure of Tac QD in 27 stable kidney transplant recipients after conversion from fasting to fed ingestion of the drug.

\section{Materials and methods}

\section{Study population}

All stable kidney transplant recipients already participating in a clinical follow-up study $^{9}$, and treated with Tac QD under fasted conditions were eligible for participation in this sub-study. Thereby, patients met the inclusion/exclusion criteria of the main study. ${ }^{9}$ For the sub-study, patients were specifically obliged to have a serum creatinine level $<265 \mu \mathrm{mol} / \mathrm{l}$ at enrolment. 
The total daily dose of each immunosuppressive medication or any other concomitant medication had remained unchanged for at least 7 days prior to enrolment of the sub study. Patients who had been treated for any rejection episode within 90 days prior to enrolment, any rejection episode within the last 6 months that had required antilymphocyte antibody therapy or more than 2 rejection episodes the last 12 months were excluded as were patients with significant liver disease. Medication or substances known to interfere with tacrolimus metabolism taken within 28 days prior to enrolment were prohibited. The patients were capable of understanding the purpose and risks of the sub-study, had been fully informed and had given written informed consent.

\section{Study design}

This study was an open-label, single-centre one way cross-over pharmacokinetic (PK) study and was approved by the local medical ethical board. Following enrolment, all patients remained on all their medication and the same dosing regimen throughout the study. If any change of regimen was clinically necessary at any time during the sub-study, the patient had to be withdrawn.

The study was divided into four periods including five scheduled visits for screening and control purposes (Figure 5.1). Patient enrolment took place on day 1 after giving written informed consent. During period 1 (screening period, 7-14 days), period 2 (7 days) and period 4 (7 days), Tac QD capsules were taken at $8.00 \mathrm{hrs}$. in the morning under fasted conditions. Fasted condition in our study meant no food intake after midnight and ingestion of Tac QD at least 1 hour before or 2-3 hours after a meal. Tacrolimus was supplied as $0.5,1$ and $5 \mathrm{mg}$ capsules.

During period 3 (7 days) Tac QD capsules were taken within 5 minutes of completion of a standard continental breakfast in the morning. The standard continental breakfast consisted of two slices of bread, cheese, ham, egg, marmalade, fresh fruits or juice (grapefruit and grapefruit juice were prohibited), tea and/or coffee. This breakfast would contain $2177 \mathrm{~kJ}$ (520 kcal), consisting of $106 \mathrm{~g}$ (53\%) carbohydrates, $24 \mathrm{~g}(28 \%)$ fat and $37 \mathrm{~g} \mathrm{(19 \% )} \mathrm{of} \mathrm{proteins.}$

Patients were hospitalized for 24 hours and two 24-hour blood concentration-time profiles were collected: Profile 1 on day 7of period 2 and profile 2 on day 14 (day 7 of period 3). Each profile consisted of 10 blood samples which were drawn at 0 hours (pre-dose) and 1, 2, 3, 4, 6, 8, 10, 12 and 24 hours after dosing.

All PK related data and other information on each patient generated according to the sub study protocol were recorded in the case report file (CRF). All data regarding adverse events, study medication, concomitant (immunosuppressive) medication, rejections and hospitalizations were recorded in the main CRF of the clinical study. Whole blood tacrolimus trough levels were used as a guide to assess patient compliance during the study. 
The primary objective of this sub study was to assess the effect of food on the pharmacokinetics and relative bioavailability of orally administered Tac QD.

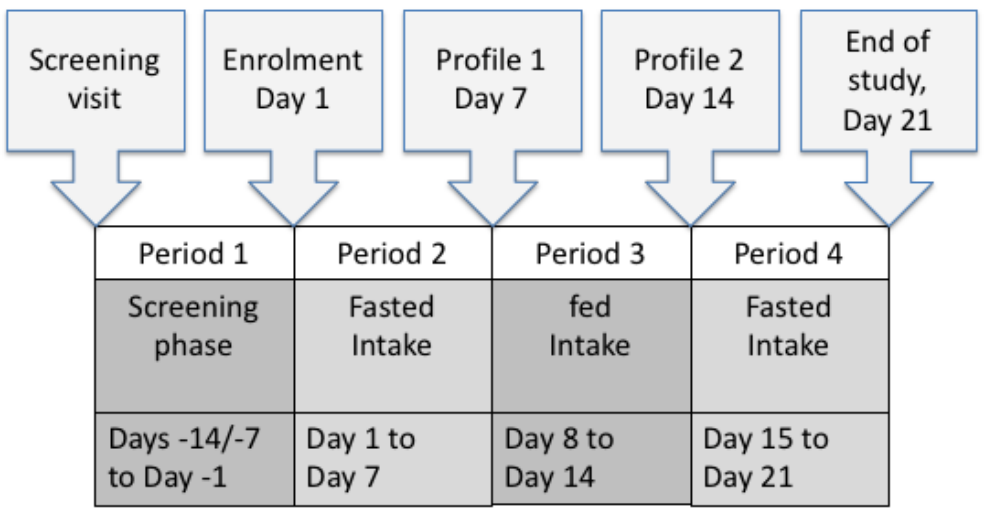

Figure 5.1 Study design.

\section{Sample handling and assay}

At each sampling time, a $2 \mathrm{ml}$ aliquot of whole blood was collected. All blood samples were drawn into pre-labelled ethylenediaminetetraacetic acid (EDTA) vacutainer tubes. The blood samples were frozen within 1 hour of withdrawal and were stored at $-20^{\circ} \mathrm{C}$ until shipping to the central laboratory, Covance Laboratories Ltd. (Harrogate, UK) for analysis.

Whole blood Tac concentrations were determined using ultra performance liquid chromatography mass spectrometry (UPLC-MS/MS) for all samples of the 24-hour blood concentration-time profiles (lower limit of quantification $0.059 \mathrm{ng} / \mathrm{ml}$ ). Wholeblood calibrators, quality control samples and study samples were thawed and 1-ml aliquots were taken. Analog internal standard of tacrolimus (FR900520; $20 \mu \mathrm{l}$, $50 \mathrm{ng} / \mathrm{ml}$ ) was added and mixed briefly. Aliquots were extracted using protein precipitation and solid-phase extraction using C18 200-mg/3-ml extraction cartridges. Elutes were evaporated to dryness under a stream of nitrogen at $40^{\circ} \mathrm{C}$, and residues were re-dissolved in a $50: 50 \mathrm{mix}(\mathrm{v} / \mathrm{v})$ of acetonitrile and water, mixed and centrifuged, before being submitted for UPLC/MS/MS. Analysis of PK samples was performed working on validated procedures in compliance with the Principles of Good Laboratory Practice as described earlier. ${ }^{7}$ 


\section{Pharmacokinetic analysis}

The PK analysis was conducted using WinNonlin Enterprise Version 4.0.1 (Pharsight Corporation, Mountain View, California, USA). The PK parameters determined were:

- area under the blood concentration-time curve from time zero to 24 hours $\left(\mathrm{AUC}_{0-24}\right)$,

- maximum observed concentration $\left(C_{\max }\right)$

- concentration at 24 hours post-dose $\left(\mathrm{C}_{24}\right)$

- time to reach maximum concentration $\left(t_{\max }\right)$

- geometric mean ratio of fed/fasted $\mathrm{AUC}_{0-24}$

\section{Statistics}

\section{Sample Size}

Based on previous experience with similar studies and the EMEA guidelines on the investigation of bioequivalence ${ }^{10}, 24$ patients with 2 complete evaluable profiles was considered to be adequate for investigating the effect of food on the exposure of Tac. So, no formal sample size calculation was performed. Complete profiles were required; if patients had a missing blood sample they were regarded as non-evaluable.

\section{Pharmacokinetics}

The PK endpoints $A \cup C_{0-24}, C_{\max }, C_{\min }$ and $t_{\max }$ were derived from the blood concentration-time profiles by using non-compartmental methods (trapezoidal rule for the Area under the blood concentration-time curve (AUC)). Any comparison of PK parameters was done for assumed log-normal distribution for concentration-related parameters at the two-sided $10 \%$ level of significance corresponding to two-sided $90 \%$ confidence intervals derived from an appropriate ANOVA for repeated measurements using an mixed model of the form:

Response $=$ Subject + Food state + random error .

In the above model, the term 'Subject' was fitted as a random effect and the term 'Food State' was fitted as a fixed effect. Least square means were calculated for the fed and fasted states. Mean differences between the fed and fasted state were calculated. The residual variance from the mixed model was used to calculate $90 \%$ confidence intervals $(\mathrm{Cl})$ for the difference between the fed and the fasted states. These values were back transformed to give geometric least square means, a point estimate and $90 \% \mathrm{Cl}$ for the ratio of the fed relative to the fasted state.

We arbitrarily judged a $20 \%$ change in $\mathrm{AUC}_{0-24}$ or $\mathrm{C}_{24}$ a clinically significant change. The $20 \%$ cut-off value is based on the reported coefficients of variation for tacrolimus trough levels at $5 \mu \mathrm{g} / \mathrm{l}$ and for $\mathrm{AUC}_{0-24}{ }^{4,11}$ 


\section{Results}

The study group (Table 5.1) consisted of 17 male and 10 female patients. All patients were white with the exception of 1 Asian male participant. Their mean age was 49 (range 31-63) years with a median time since transplantation of 8.3 (range 5.4-15.6) years. Additional immunosuppression consisted of mycophenolate mofetil in 5 patients and prednisolone in 1 patient. The remaining 21 patients were on tacrolimus monotherapy. Mean tacrolimus dose $/ \mathrm{kg}$ body weight was $0.06 \mathrm{mg} / \mathrm{kg}$ (range $0.01-0,2 \mathrm{mg} / \mathrm{kg}$ ). All patients completed two 24-hour blood concentration profiles for Tac, after administration of Tac QD under fasted and fed conditions. There were no missing data.

Table 5.1 Subject demographics.

\begin{tabular}{|c|c|}
\hline \multicolumn{2}{|l|}{ Sex } \\
\hline Male & 17 \\
\hline Female & 10 \\
\hline \multicolumn{2}{|l|}{ Race } \\
\hline white & 26 \\
\hline Asian & 1 \\
\hline \multicolumn{2}{|c|}{ Years since transplantation (years) } \\
\hline Mean & 8.3 \\
\hline range & $5.4-15.6$ \\
\hline \multicolumn{2}{|l|}{ Age (years) } \\
\hline Mean & 49 \\
\hline range & $31-63$ \\
\hline \multicolumn{2}{|l|}{ Weight (kg) } \\
\hline Mean & 78 \\
\hline range & $58-100$ \\
\hline \multicolumn{2}{|l|}{ Daily dose Tacrolimus QD (mg) } \\
\hline Mean & 4.3 \\
\hline range & $1-15$ \\
\hline \multicolumn{2}{|c|}{ Daily dose Tacrolimus QD (mg/kg) } \\
\hline Mean & 0.06 \\
\hline range & $0.01-0.2$ \\
\hline \multicolumn{2}{|l|}{ Immunosuppression } \\
\hline Tacrolimus monotherapy & 21 \\
\hline \multicolumn{2}{|l|}{ Co-immunosuppression } \\
\hline Mycophenolate Mofetil & 5 \\
\hline Prednisolone & 1 \\
\hline \multicolumn{2}{|l|}{ Creatinine $(\mathrm{mcmol} / \mathrm{l})$} \\
\hline Mean & 141 \\
\hline range & $82-250$ \\
\hline
\end{tabular}

Whole blood concentrations of tacrolimus following administration of Tac QD in the fasted and fed state are presented in Figure 5.2. Summary statistics for PK parameters of the patient group are presented in Table 5.2. All PK parameters $\left(A \cup C_{0-24}, C_{\max }\right.$, and 
$\mathrm{C}_{24}$ ) showed a decrease in the fed compared to the fasted state $(p<0,001$ for all parameters). $T_{\max }$ was prolonged for 1 hour under fed conditions with a flattened curve at peak concentrations (Figure 5.2). The $90 \% \mathrm{Cl}$ for the geometric mean ratio $(90 \% \mathrm{Cl})$ of $\mathrm{AUC}_{0-24}$ and $\mathrm{C}_{24}$ were $0.86(0.81-0.91)$ and 0.87 (0.74-0.90), respectively.

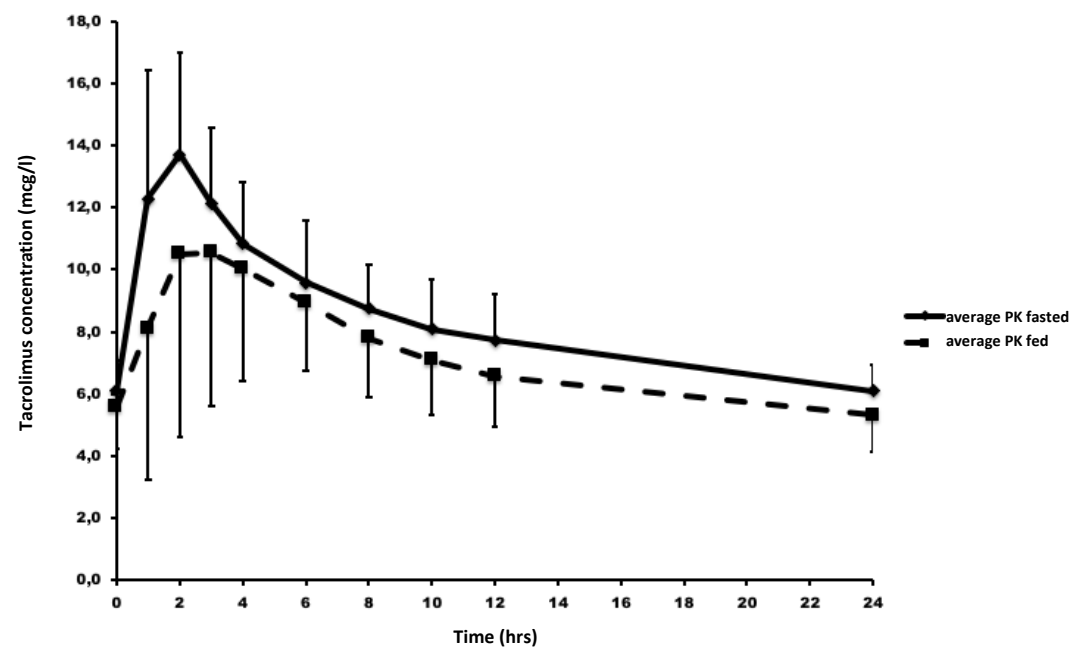

Figure 5.2 24-hour blood concentration-time profiles of tacrolimus following ingestion of Tac QD under fasting (solid line) and fed (dashed line) conditions. Concentrations are mean whole blood tacrolimus concentrations; error bars reflect the standard deviation.

Table 5.2 Summary of PK parameters of all subjects.

\begin{tabular}{lcccc}
\hline Parameter & $\begin{array}{c}\text { Tac QD } \\
\text { Fasted } \\
\text { (range) }\end{array}$ & $\begin{array}{c}\text { Tac QD } \\
\text { fed } \\
\text { (range) } \\
\mathrm{N}=27\end{array}$ & $\begin{array}{c}\text { Ratio of geometric } \\
\text { means } \\
(90 \% \mathrm{Cl}) \text { fed:fasted }\end{array}$ & ${ }^{{ }^{a} p}$ \\
\hline $\mathrm{AUC}_{0-24}(\mu \mathrm{g} . \mathrm{h} / \mathrm{l})$ & 201 & 173 & 0.86 & \\
& $(140-264)$ & $(130-341)$ & $(0.81-0.91)$ & $<0.001$ \\
$\mathrm{C}_{24}(\mu \mathrm{g} / \mathrm{l})$ & 6.1 & 5.3 & 0.87 & $<0.001$ \\
& $(4.5-7.9)$ & $(3.7-9.3)$ & $(0.82-0.92)$ & \\
$\mathrm{C}_{\max }(\mu \mathrm{g} / \mathrm{l})$ & 14.1 & 11.5 & 0.81 & 0.001 \\
& $(10.1-24.5)$ & $(6.7-29.8)$ & $(0.74-0.90)$ & $<0.001$ \\
${ }^{\mathrm{b}} \mathrm{T}_{\max }(\mathrm{h})$ & 2.0 & 3.0 & $\mathrm{NC}$ & \\
\hline
\end{tabular}

Geometric mean (range) are presented. $\mathrm{N}=$ total number of patients; $N C=$ not calculated; ${ }^{\mathrm{a}} \mathrm{P}$ for ANOVA; ${ }^{\mathrm{b}}$ Median (range). 
Individual changes in $\mathrm{AUC}_{0-24}$ ranged from -38 to $+29 \%$ (Figure 5.3). In 16 out of 27 patients (59\%) the change of $\mathrm{AUC}_{0-24}$ in was less than $20 \%$ (Figure 5.4). Two patients (7\%) had an increase in $\mathrm{AUC}_{0-24}$ of 20 and $29 \%$, respectively. These patients had an increase in $\mathrm{C}_{\max }$ under fed conditions of 52 and $89 \%$, respectively and were in the highest quartile of weight-corrected tacrolimus dose $(0.08$ and $0.14 \mathrm{mg} / \mathrm{kg})$. Nine patients (33\%) had a more than $20 \%$ decrease in $\mathrm{AUC}_{0-24}$ after ingestion of the drug together with the continental breakfast. Within these patients, the lowest fasted $\mathrm{AUC}_{0-24}$ was $173 \mu \mathrm{g} . \mathrm{h} / \mathrm{l}$ and four patients had fasted AUCs of about $238 \mu \mathrm{g} . \mathrm{h} / \mathrm{l}$ (highest quartile).

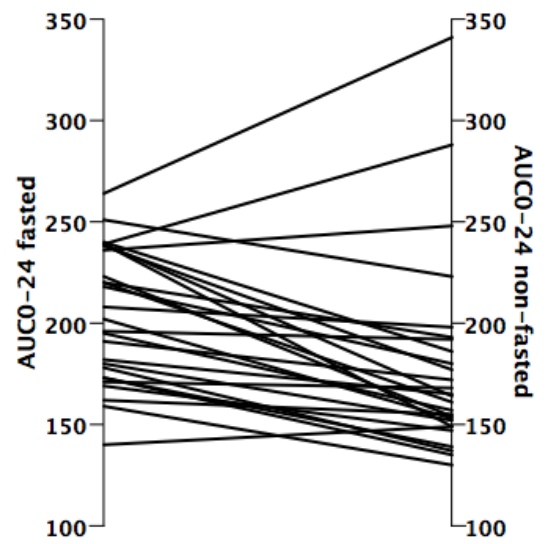

Figure 5.3 Individual changes in AUC0-24 after conversion from the fasted to the fed state.

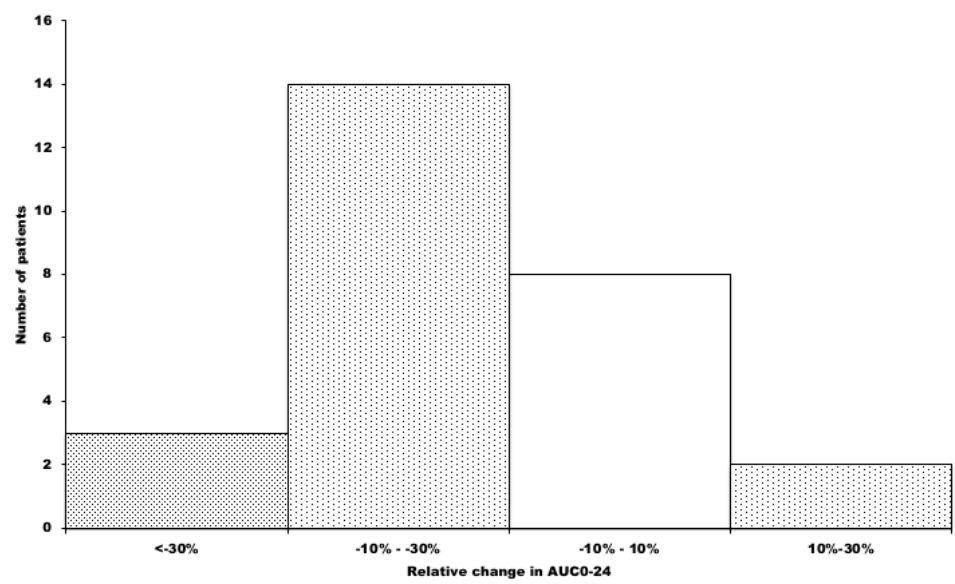

Figure 5.4 Histogram of relative changes in $\mathrm{AUC}_{0-24}$ after conversion from fasted to fed ingestion of Tac QD. 
The change in $\mathrm{C}_{24}$ after conversion to fed conditions ranged from $-39 \%$ to $+36 \%$. The majority had a non-significant change of less than $20 \%$. The targeted trough concentrations ranged between 4 and $8 \mu \mathrm{g} / \mathrm{l}$. One patient showed an increase from $6,8 \mu \mathrm{g} / \mathrm{l}\left(75^{\text {th }}\right.$ percentile) to $9.3 \mu \mathrm{g} / \mathrm{l}$ ( $>20 \%$ increase). Seven patients (26\%) showed a more than $20 \%$ decrease in $\mathrm{C}_{24}$ (five of whom had also shown a more than $20 \%$ decline in $\mathrm{AUC}_{0-24}$ ) after ingestion of the drug together with the continental breakfast. One patient declined from $5.7 \mu \mathrm{g} / \mathrm{l}$ to $3.7 \mu \mathrm{g} / \mathrm{l}$, whereas the remaining six patients remained above $4 \mu \mathrm{g} / \mathrm{l}$.

The correlation between $\mathrm{AUC}_{0-24}$ and $\mathrm{C}_{24}$ under fed conditions was good and numerically higher than under fasting conditions ( $R=0.89$ and $R=0.77$, respectively) (Figure 5.5). In the therapeutic trough level target range, the difference between both slopes was minimal (Figure 5.5).

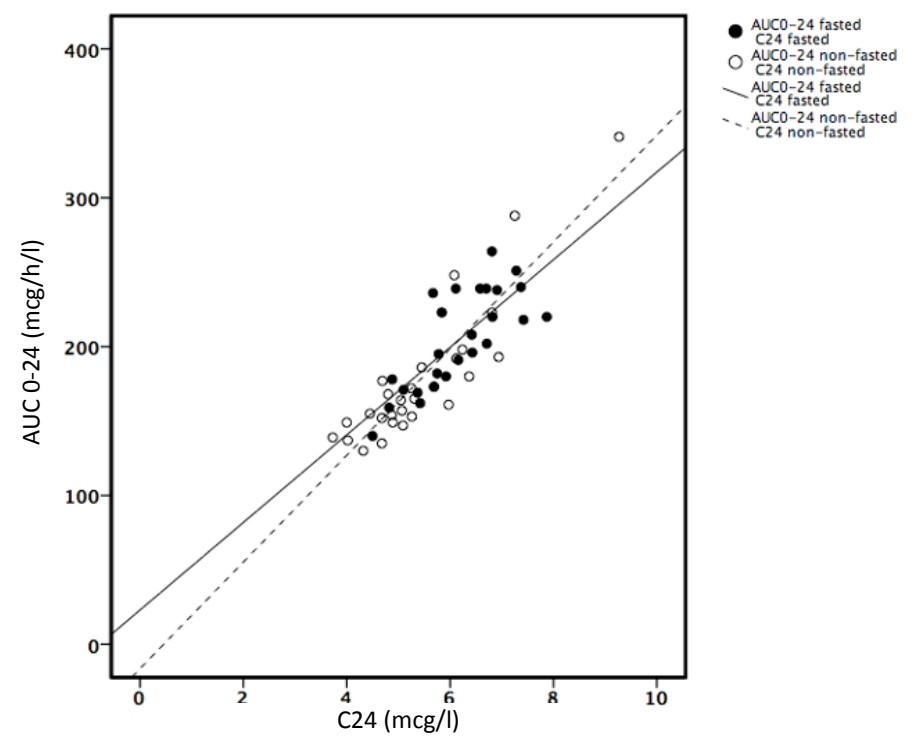

Figure 5.5 Correlations between $\mathrm{AUC}_{0-24}$ and $\mathrm{C}_{24}$ for tacrolimus following administration of Tac QD under fasted and fed conditions. The regression equation equal:

- for fasting conditions: $\mathrm{AUC}_{0-24}=29.426 * \mathrm{C}_{24}+22.992$ (correlation coefficient $\mathrm{R}=0.78$ ).

- For fed conditions: $\mathrm{AUC}_{0-24}=35.851 * \mathrm{C}_{24}-16.479$ (correlation coefficient $\mathrm{R}=0.89$ ).

\section{Safety}

There were neither episodes of biopsy proven acute rejection during the conduct of the study nor any other (severe) adverse events. None of the patients had a doseadjustment. 


\section{Discussion}

This pharmacokinetic study was conducted to assess the effect of a standard continental breakfast on the relative bioavailability of orally administered tacrolimus modified release formulation, Tac QD, in (stable) kidney transplant recipients. To our knowledge this is the first PK study on this matter for the newer tacrolimus formulation in transplant patients. Relevant clinical PK parameters like $\mathrm{AUC}_{0-24}$ and $\mathrm{C}_{24}$ showed an average $13-14 \%$ decrease according to the geometric mean ratios. We also observed a 1-hour prolongation of the $T_{\max }$ when Tac QD is administered together with the breakfast compared with administration under fasting conditions. Although the mean ratio of $\mathrm{AUC}_{0-24}$ is just below 0.9 , there was a large spread in individual changes in $\mathrm{AUC}_{0-24}$ (Figures 5.2, and 5.3). Therefore, in clinical practice we would advise to monitor tacrolimus trough levels one week after the patient is converted to fed ingestion of tacrolimus. As the relationship between tacrolimus trough level and $\mathrm{AUC}_{0-24}$ was comparable in the therapeutic trough level range under fasting and fed conditions, the trough level can be used in clinical guidance of tacrolimus exposure under these conditions for trough levels between approximately 4 and $8 \mu \mathrm{g} / \mathrm{l}$, as observed in our study.

The paper on the scientific discussion on the product specifications cites a company driven study on the relationship between the timing of a meal and subsequent blood levels of tacrolimus in 23 healthy individuals. ${ }^{8}$ The study showed that in the presence of food there was an increase of approximately $80 \%$ in time to maximum whole blood tacrolimus concentration and a reduction of approximately $20 \%$ in maximum whole blood tacrolimus concentration and a reduction of $26 \%$ in AUC in the presence of food. We find the same general tendency, but with less outspoken average decreases, probably due to the higher interindividual range of changes (i.e. also patients with a significant increase in $\mathrm{AUC}_{0-24}$ and $\mathrm{C}_{24}$ ).

In another earlier study in healthy individuals, a high fat meal also significantly reduced the rate and extent of tacrolimus absorption relative to that in the fasted state. ${ }^{8}$ The mean $\mathrm{AUC}_{0-24}$ was reduced by $25 \%$, which is a stronger decline than that observed in our study. The higher fat content of food used in this population may be responsible for this difference since a high fat meal has a stronger impact on tacrolimus bioavailability than a continental breakfast as has been shown by Bekersky et al.. ${ }^{1}$ The reason for this was probably fat-induced delayed gastric emptying.

In the Bekersky study, the low-fat meal, which is comparable to that of our study, reduced the oral bioavailability of the BID formulation by $25 \% .{ }^{1}$ This also is more than the reduction in AUC in the current study and could be related to a more pronounced Tac QD absorption in the distal gastro-intestinal tract. ${ }^{12}$ The same mechanism was also postulated earlier for kidney transplant patients: in a study on the effect of continental breakfast on PK parameters of the BID formulation at different episodes 
post transplantation, at one year after transplantation the AUCs did not differ significantly after drug ingestion with food. ${ }^{2}$ Because Tac QD has been shown to be absorbed in the more distal part of the gastro-intestinal tract the impact of food should be diminished for Tac QD compared with these data. Because this is not the case, we postulate that other variable factors like activity of the drug efflux pump $p$ Glycoprotein might play a role.

Despite a $14 \%$ decrease in average $A U C_{0-24}$ with a minimum $A U C_{0-24}$ of $130 \mu \mathrm{g} . \mathrm{h} / \mathrm{l}$ in the fed state, of notice is, that all but one fed trough levels remained within the therapeutic range. In addition, no adverse events like biopsy proven rejection occurred. From clinical data, there is no clear consensus what the target $\mathrm{AUC}_{0-24}$ for Tac QD should be. An earlier consensus report on the twice daily Prograf formulation mentions that due to the limited number of studies recommending a target AUC, it is difficult to reach any consensus for a target. ${ }^{13}$ The lowest $A U C_{0-24}$ in our study is within the range of AUCs reported in earlier conversion studies of Tac QD with similar safety profiles after conversion.,

Surprisingly, two of the 27 patients had a substantial (>20\%) increase in $\mathrm{AUC}_{0-24}$ when the drug was ingested together with a continental breakfast. A former study about the influence of breakfast on tacrolimus trough levels using the twice daily Prograf formulation also showed an increase $>20 \%$ in tacrolimus trough levels in 3 out of the 27 patients. ${ }^{4}$ Therefore, our observation seems to be valid. There were no patient characteristics that could be linked to this observation.

The potential influence of the Cyp3A5 genotype was investigated in a post-hoc analysis. For this purpose, genetic analysis was performed from a venous blood sample each patient provided at the screening visit of this study and was analyzed as described before. ${ }^{14}$ The ratio in $A_{U C} C_{0-24}$ fed versus fasting was numerically higher in recipients with Cyp3A5*1/*3 $(n=5)$ compared to the patients with Cyp3A5 *3/*3 $(n=22)$ : respectively 0.936 and 0.845 . The same trend was found for $C_{\min }, C_{24}$ and $C_{\max }$ (data not shown). It seems that conversion to fed intake has less impact on the tacrolimus exposure in patients with Cyp3A5*1/*3. In our study, we did not have patients with Сур $3 A 5{ }^{*} 1 /{ }^{*} 1$. This very intriguing observation needs confirmation in a larger population.

In conclusion, we found a modest, clinically not-significant decrease in $\mathrm{AUC}_{0-24}, \mathrm{C}_{\max }$, $C_{24}$ and a prolongation of $T_{\max }$ in the majority of patients when Tac QD is ingested with standard continental breakfast. A limitation of this study is that we investigated only the influence of a standard continental breakfast, which has a low-fat content, on the tacrolimus exposure. The influence of a 'high-fat' breakfast in kidney transplant recipients is unknown. In the end, fed ingestion of the drug could be more convenient for the patient and may promote therapy adherence. As we have found that ingestion of Tac QD together with the standard continental breakfast results into a wide interindividual change in exposure in approximately $40 \%$ of the patients, we advise to monitor tacrolimus trough level at one week after allowing the patient to ingest Tac 
QD together with food, with the precaution that our observations may apply for the trough concentration range of $4-8 \mu \mathrm{g} / \mathrm{l}$.

\section{Acknowledgement}

This trial was funded by Astellas Pharma Europe Ltd. 2000 Hillswood Drive Chertsey KT16 ORS United Kingdom. 


\section{References}

1. Bekersky I, Dressler D, Mekki QA Effect of low- and high-fat meals on tacrolimus absorption following $5 \mathrm{mg}$ single oral doses to healthy human subjects J Clin Pharmacol. 2001;41:176-182.

2. Christiaans $M$, van Duijnhoven E, Beysens $T$, et al. Effect of breakfast on the oral bioavailability of tacrolimus and changes in pharmacokinetics at different times posttransplant in renal transplant recipients Transplant Proc. 1998;30:1271-1273.

3. van Duijnhoven E, Christiaans $M$, Undre N, et al. J The effect of breakfast on the oral bioavailability of tacrolimus in diabetic and nondiabetic patients before transplantation Transplant Proc. 1998;30: 1268-1270.

4. van Duijnhoven EM, Christiaans MH, Boots JM, et al. Evidence that fasting does not significantly affect trough levels of tacrolimus in stable renal transplant recipients Transplant Proc. 2002;34:1723-1725.

5. Alloway R, Steinberg S, Khalil K, et al. Conversion of stable kidney transplant recipients from a twice daily Prograf-based regimen to a once daily modified release tacrolimus-based regimen Transplant Proc. 2005;37:867-870.

6. Kramer BK, Charpentier B, Backman L, et al. Tacrolimus once daily (ADVAGRAF) versus twice daily (PROGRAF) in de novo renal transplantation: a randomized phase III study Am J Transplant. 2010;10: 2632-2643.

7. van Hooff J, Van der Walt I, Kallmeyer J, et al. Pharmacokinetics in stable kidney transplant recipients after conversion from twice-daily to once-daily tacrolimus formulations Ther Drug Monit. 2012;34: 46-52.

8. European Medicines Agency. Advagraf: EPAR-Scientific Discussion [web site] 2007. Available at: http://www.ema.europa.eu/docs/en_GB/document_library/EPAR_-_Scientific_Discussion/human/ 000712/WC500022237.pdf.

9. van Hooff JP, Alloway RR, Trunecka P, et al. Four-year experience with tacrolimus once-daily prolonged release in patients from phase II conversion and de novo kidney, liver, and heart studies Clin Transplant. 2011;25:E1-12.

10. European Medicines Agency. Note for Guidance on the Investigation of Bioavailability and Bioequivalence London, 2010.

11. Shuker N, van Gelder T, Hesselink DA Intra-patient variability in tacrolimus exposure: causes, consequences for clinical management Transplant Rev. 2015;29:78-84.

12. European Medicines Agency. Advagraf: EPAR-Product information [web site] 2009. Available at: http://www.ema.europa.eu/ema/index.jsp?curl=pages/medicines/human/medicines/000712/human _med_000629.jsp\&mid=WC0b01ac058001d124.

13. Wallemacq $\mathrm{P}$, Armstrong $\mathrm{VW}$, Brunet $\mathrm{M}$, et al. Opportunities to optimize tacrolimus therapy in solid organ transplantation: report of the European consensus conference Ther Drug Monit. 2009;31: 139-152.

14. Stifft F, Stolk LM, Undre N, et al. Lower variability in 24-hour exposure during once-daily compared to twice-daily tacrolimus formulation in kidney transplantation Transplantation. 2014;97:775-780. 


\section{Chapter 6}

Lower variability in 24-hour exposure during once-daily compared with twice-daily tacrolimus formulation in

kidney transplantation

F. Stifft, L.M.L. Stolk, N. Undre, J.P. van Hooff, M.H.L. Christiaans

Transplantation 2014;97(7):775-80 


\section{Abstract}

\section{Introduction}

Tacrolimus has originally been registered as a twice-daily formulation (Prograf, Tac BID), although, a once-daily formulation (Advagraf, Tac QD) is also available. A reduced intrapatient variability of $\mathrm{Tac} \mathrm{C}_{\mathrm{min}}$, a surrogate marker for 24-hour drug exposure $\left(A \cup C_{0-24}\right)$ has been suggested. The variability of $A \cup C_{0-24}$ has never been studied prospectively yet. The purpose of this study was to investigate the change in intrapatient variability of Tac $\mathrm{AUC}_{0-24}$ after conversion from Tac BID to Tac QD.

\section{Methods}

Forty renal transplant patients on Tac BID were converted on a 1:1 (mg:mg) basis to Tac QD in an investigator-driven comparative pharmacokinetic (PK) study. $\mathrm{AUC}_{0-24}$ was determined 5 times before and after conversion. Duplicate samples were collected by the patients themselves using the Dried Blood Spot method. The main outcome measure is the change in intrapatient variability of $A U C_{0-24}$ expressed as coefficient of variation (CV). Moreover, the influence of Cyp3A5 genotype polymorphism on change in CV was studied.

\section{Results}

In total, $400 \mathrm{AUC}_{0-24}$ were available for analysis. Conversion to Tac QD resulted in a significant improvement of intrapatient CV from $14.1 \%$ to $10.9 \%(p=0.012)$. Patients with the Cyp3A5*1/*3 genotype $(n=11)$ had a numerically larger improvement in CV than patients with the CYP3A $5 * 3 / * 3$ genotype.

\section{Conclusion}

Intra-patient CV of Tac $\mathrm{AUC}_{0-24}$ improved after conversion of Tac BID to Tac QD in stable renal transplant patients, especially in patients with the CYP3A5*1/3 genotype. Given the very strict protocol of this PK study this improvement is most likely due to different intrinsic PK properties of Tac QD and Tac BID. 


\section{Introduction}

Tacrolimus (Tac) is the cornerstone immunosuppressive drug in solid organ transplantation and has been available as an immediate release formulation administered twice daily (Prograf, Tac BID) for almost 20 years. More recently, a oncedaily prolonged release formulation (Advagraf, Tac QD) has been made available. The efficacy and safety profile of Tac QD is comparable to that of Tac BID after conversion to Tac QD in stable kidney transplant recipients. ${ }^{1,2}$

Tacrolimus has a narrow therapeutic window and high interpatient variability in its bioavailability. Therefore, therapeutic drug monitoring (TDM) of exposure is mandatory in order to ensure that patients are maintained in a narrow predefined therapeutic exposure window. ${ }^{3}$ The area under the 24-hour blood concentration-time curve $\left(A \cup C_{0-24}\right)$ is presumed to better reflect exposure to the drug than predose concentrations or other single-time measurements. In clinical practice, this is not practically feasible and therefore, the trough level $\left(C_{\min }\right)$ that correlates well with the $\mathrm{AUC}_{0-24}$ is used as a surrogate marker for exposure. This relationship between $\mathrm{C}_{\min }$ and the $\mathrm{AUC}_{0-24}$ is similar for Tac QD and Tac BID. ${ }^{1,2,4}$

There are indications that high intrapatient (within-patient) variability (CV) in Tac BID $\mathrm{C}_{\min }$ is a risk factor for poor long-term outcome after kidney transplantation. ${ }^{5}$. Earlier safety and tolerability or PK studies have suggested that $\mathrm{CV}$ for $\mathrm{C}_{\min }$ was lower during Tac QD when compared to Tac BID in stable kidney transplant patients. ${ }^{1,2}$ An explanation for this lower CV is that once-daily dosing of tacrolimus leads to an improved adherence. ${ }^{6}$ However, improvement of intrinsic PK properties of Tac QD might result in a lower $\mathrm{CV}$ as well.

Alloway et al. reported a greater decrease in CV of tacrolimus exposure with Tac QD versus Tac BID for African-American patients compared to white patients. ${ }^{1}$ Similarly, Wu et al. report a decrease of $C V$ in $C_{\min }$ after conversion to Tac $Q D$ in a Chinese population. ${ }^{7}$ This decrease in $\mathrm{CV}$ might be linked to the higher prevalence of Cytochrome P450 3A5*1 (Cyp3A5*1) allele in African Americans and Chinese. It is expressed in more than $70 \%$ among African Americans, and $30 \%$ in Chinese in contrast to only $10 \%$ in whites. ${ }^{8-10}$

To our knowledge, intrapatient CV of the Tac exposure $\left(\mathrm{AUC}_{0-24}\right)$ - both of Tac BID and Tac QD - has not yet been investigated prospectively in stable renal transplant recipients. Therefore, a prospective PK study was designed in which CV of the $\mathrm{AUC}_{0-24}$ in stable renal transplant patients was compared before and after conversion from Tac BID to Tac QD. Moreover, the influence of the Cyp450 3A5 polymorphism on the change of CV after conversion was analyzed. 


\section{Methods}

\section{Patients}

All patients 18 years or older with a stable renal allograft function six months prior to inclusion and on stable Tac BID dosages were eligible for participation in our study. Because we were particularly interested in the Cyp3A5*1 genotype, patients with a daily dose at least twice as high as the average daily dose (i.e. $6 \mathrm{mg}$ or more) were regarded as potential carriers of the Cyp3A $5 * 1$ genotype and were preferentially selected. Patients were excluded if they had any organ other than kidney transplanted, had active malignancy, had renal replacement therapy (RRT), had signs of infection shortly before inclusion, or have chronic diarrhoea. Patients were enrolled after they had given their informed consent. The study had been approved by the local medical ethics committee (METC 09-2-040) and was registered in the EUDRA CT trial register (registration no. 2009-010400-28).

\section{Study design}

The study was an investigator-driven, single centre, open label, prospective twosequence, conversion comparative study, in which PK parameters where evaluated with respect to their variability before and after converting from Tac BID to Tac QD. The study period started with a run-in period of two weeks during which the patients were instructed to take the Tac BID under fasting conditions, that is, one hour before or two hours after a meal. From the third week on, five weekly 24-hour blood concentration-time to determine $\mathrm{AUC}_{0-24}$ were taken (Figure 6.1), and if one of the profiles was not evaluable (see below), an additional profile was taken. After five evaluable profiles had been taken, patients were converted to Tac QD on a 1:1 (mg:mg) basis. After a stabilisation period of two weeks, five additional evaluable 24-hour AUC profiles at weekly intervals were taken in the second phase of the study (Figure 6.1). One week after conversion, a venous tacrolimus trough level was taken and Tac QD dosage was adjusted if the trough level was $<3,5 \mu \mathrm{g} / \mathrm{l}$. Sampling of the first blood concentration-time profile was postponed up to 2 weeks after the last Tac QD adjustment.

\section{Sample size}

Because no numerical data of a change in intrapatient variability in $24 \mathrm{~h}$ drug exposure were available, no formal statistical power analysis could be performed. The study population was set at 40 patients based on common practice in other PK studies. ${ }^{12}$ 


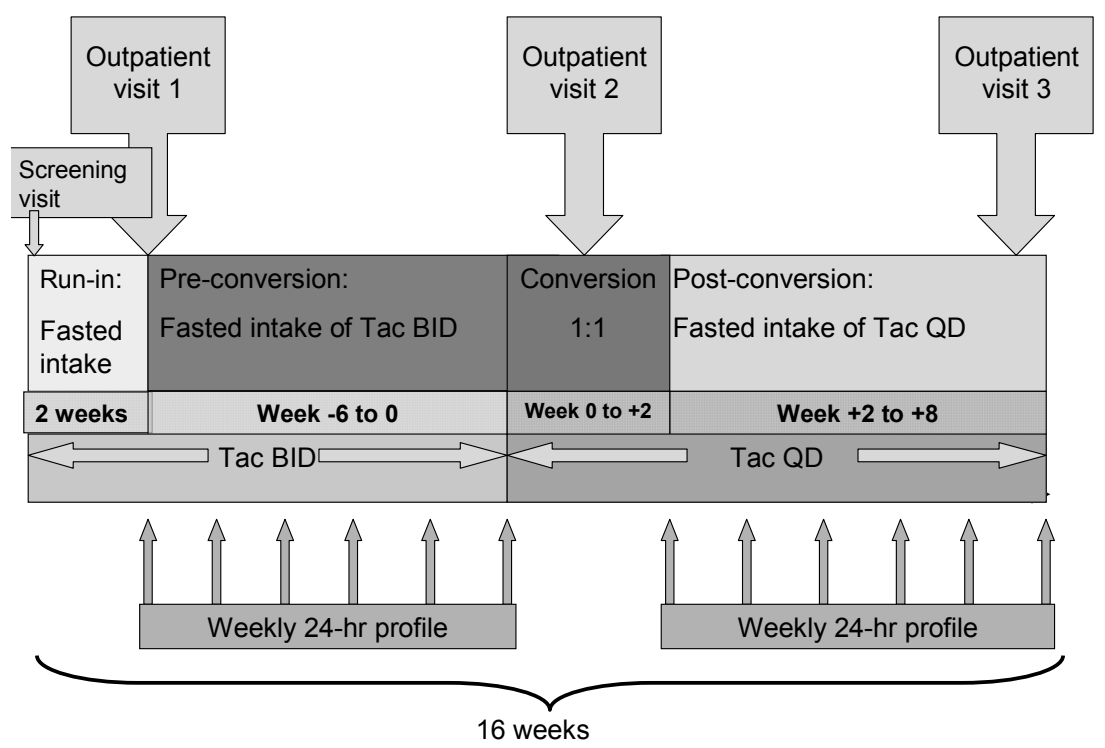

Figure 6.1 Study time table.

\section{Study parameters/endpoints}

The primary objective of this study was to evaluate and compare the intrapatient variability in $\mathrm{AUC}_{0-24}$ of $\mathrm{Tac} B I D$ and $\mathrm{Tac} Q \mathrm{QD}$ in stable renal transplant patients. Secondary endpoints were correlation of the PK profiles with the Cyp3A5 genotype and comparison of the intrapatient variability of the trough levels $\left(C_{\min }\right)$.

\section{Assessments and assay}

A total number of five AUC's were arbitrarily considered adequate for the assessment of the intrapatient variability for each formulation. Each PK profile consisted of 8 blood samples that were collected before dosing and at 2, 4, 8, 12, 14, 16 and at 24 hours after dosing by means of the Dried Blood Spot method (DBS). This 8-point 24-hour PK profile was assumed to give a valid AUC for both Tac BID and Tac QD.

As for Tac BID, earlier reports guarantee prediction of the $A U C_{0-12}$ when samples at $t=0$ hour, $\mathrm{t}=2$ hours and $\mathrm{t}=4$ hours are included. ${ }^{15}$ For the $\mathrm{AUC}_{0-24}$, we added the corresponding time points after the evening dose, namely, $t=12$ hours, $t=14$ hours and $\mathrm{t}=16$ hours. We extended this 6 -point strategy to 8 points by adding $\mathrm{t}=8$ hours and $\mathrm{t}=24$ hours, to get more information about the elimination phase. For the patient's convenience, we did not add $\mathrm{t}=20$ hours. 
As for the Tac QD profiles, our centre has previously participated in a local board approved phase 3 trial (METC code 03-102; Eudra CT no. 2005-005714-20) in which 27 stable renal transplant patients provided a venous 24 -hour PK profile under fasting conditions. This profile consisted of 10 time points at $\mathrm{t}=0,1,2,3,4,6,8,10,12$ and 24 hours. The 8-point AUC was then validated by means of linear regression: this model revealed a maximum $R^{2}$ of 0.995 when the samples at $t=2,4,8,12$, and 24 hours were included. An AUC was regarded as adequate in case at least 6 valid blood spot samples were available, and if none of the above-mentioned crucial sample nor the trough level were missing.

If a profile did not fulfil these criteria it had to be repeated one week later. In addition, patients visited our outpatient clinics before the start of the study, one week after conversion and at the end of the study (Figure 6.1). During these outpatient visits regular venous blood and urine samples were drawn for routine post-transplantation medical care and safety reasons, including determination of a venous tacrolimus $C_{\min }$. These data were not included in the PK analysis. To rule out potential non-compliance medication intake had to be registered and was controlled by pill count for both formulations.

The patients themselves collected the required blood samples by means of the DBS method at home with strict recording of dosing and sampling time points. This method has been described and validated before. ${ }^{11}$ In brief, finger prick blood samples were collected using a contact-activated lancet (Becton, Dickinson and Company, Franklin Lakes, NJ, USA). The first two drops were collected from the lateral part of the fingertip to fill two $8 \mathrm{~mm}$ pre-marked circles, for duplicate sampling, on the sampling paper (item no. 10535097, Whatman, Schleicher and Schuell, Dassel, Germany). The blood spots were allowed to dry at room temperature for at least ten minutes before packaging and storage in a sealed plastic bag containing silicone granules. After completion of the 24-hour profile, all samples were collectively sent by regular post to our laboratory where they were stored at $4^{\circ} \mathrm{C}$ until further analysis. It has been proven that the tacrolimus in dry blood spot is stable during postal transport. ${ }^{16,17}$ Before the start of the study, patients were trained in blood spot sampling and the study was initiated only after all patients had repeatedly sent in a blood spot sample that had passed the quality control.

Each of the two blood spots on the sampling paper was analyzed on separate analytical runs, and the average value of both analyses was the reported value of the tacrolimus concentration. If the two results diverged by more than $30 \%$, this value was reported as missing and not considered. If one analytical run was invalid due to a poor quality control, we only disposed of one instead of two values. In case two runs had a missing non-crucial value at the same single time point, the average concentration at that particular time point was revealed by interpolation after natural logarithmic transformation. If more samples were missing due to analytical problems, the run was redone by analysis of the diluted extraction solution. In case a crucial 
sample was missing at a time point as defined above, the PK profile had to be repeated.

Analysis of the tacrolimus concentration started with punching out the dried blood spots and adding of an extraction solution. The prepared samples were analyzed in the same way as the routine tacrolimus assay in venous blood. The tacrolimus assay is validated according to and complies with the "Bioanalytical methods validation, Guidance for industry"18 with a lower limit of quantification of $1.0 \mu \mathrm{g} / \mathrm{I}^{11}$ The laboratory participated in the international tacrolimus proficiency testing scheme (www.bioanalytics.co.uk). The concentration of tacrolimus was determined by means of tandem mass spectrometry. Chromatography was performed using an Alliance 2795 system (Waters Ltd., Watford, UK). The mass spectrometer used in our study was a Quattro Micro (Micromass, Manchester, UK).

\section{Genotyping}

Each patient provided a venous blood sample at the screening visit of this study for genetic analysis. Genomic DNA was extracted according to the manufacturers' instructions (Qiagen, Leusden, the Netherlands). Real-time polymerase chain reaction (PCR) fluorescence resonance transfer (FRET) assays were used for genotyping with the LightCycler (Roche Diagnostics, Almere, The Netherlands).

\section{Statistics}

Demographic data are presented as mean and range, PK parameters were presented as geometric mean and $90 \%$ confidence interval $(\mathrm{Cl})$. AUC was calculated using the linear trapezoidal rule. Before statistical analysis, PK data were natural logtransformed and transformed back to the original scale for display of the data.

Intrapatient variability was calculated through the CV as follows:

$$
C V_{W R}(\%)=100 \sqrt{e^{s_{W R}^{2}}-1}
$$

where $S_{W R}$ is the within-subject standard deviation of the natural log-transformed values.

PK parameters were compared by the paired measurements T-test; Cyp3A5 polymorphism was analyzed by nonparametric tests (Wilcoxon signed ranking test for paired measurements and Mann Whitney $U$ test for non-paired measurements). $\mathrm{P}<0.05$ was considered statistically significant. Statistical analysis was performed using SPSS 16.0 for Windows, release 16.0.1. 


\section{Results}

Forty stable renal transplant patients on Tac BID (25 men, 15 women) were included in the study after giving informed consent. Mean age was 61.2 years (range 36-78) with an average time since transplantation of 4.5 years (range 1.3-13.5). They had an average estimated glomerular filtration rate of $40 \mathrm{ml} / \mathrm{min} / 1.73 \mathrm{~m}^{2}$ (range 20-66 ml/min/1.73 $\mathrm{m}^{2}$ ). Thirty-one patients were on tacrolimus monotherapy. Concomitant immunosuppression consisted of mycophenolic acid in 4 patients, sirolimus (1 mg QD) in three patients and prednisolone ( $5 \mathrm{mg}$ ) in two patients. None of the patients received more than one concomitant immunosuppressive drug. Of the 40 patients, 11 had the Сур3A5*1/*3 genotype and the other 29 had the Сур3A5*3/*3 genotype. There were no patients with the Cyp3A5*1/*1 genotype.

Each patient performed 10 PK profiles ( $n=400$ profiles), consisting of 8 duplicate samples per profile. For each profile, the duplicate samples were analyzed in separate analytical runs. In $6.3 \%$ of these runs the quality control of one of the runs was insufficient. In these instances, AUC was computed on one value per time-point. In $3 \%$ of the profiles, one noncrucial value was missed. This missing value could be estimated by interpolation. Hence, only 12 patients had to repeat one profile.

\section{Pharmacokinetics}

Relevant PK parameters and the corresponding average 24-hour profiles for both formulations before and after conversion are given in Table 6.1 and Figure 6.2. One week after conversion, in three patients the tacrolimus trough levels were $<3.5 \mu \mathrm{g} / \mathrm{l}$. In these patients, according to the protocol, the Tac doses were increased with 1, 1.5 and $2 \mathrm{mg}$ to reach a $\mathrm{C}_{\min }>4.0 \mu \mathrm{g} / \mathrm{l}$ and the profiles were made only after establishing stable $C_{\min }$. No further dose adjustments were made. Therefore, in Table 6.1, AUC $_{0-24}$ and $C_{\min }$ are also listed as dose-normalised values. There was good correlation between $\mathrm{AUC}_{0-24}$ and $\mathrm{C}_{\min }$ for both formulations with virtually identical slopes of the Tac BID and Tac QD regression curves (Figure 6.3).

\section{Intra-subject variability}

As all five-time concentration profiles $\left(\mathrm{AUC}_{0-24}\right)$ for each formulation in each patient were performed with a fixed Tac dose, all 40 patients could be included for the analyses. After conversion intrapatient $\mathrm{CV}$ of the $\mathrm{AUC}_{0-24}$ decreased significantly from 14.1 to $10.9 \%(p=0.012)$. The intrapatient $C V$ for $C_{\min }$ decreased only numerically from 15.3 to $13.7 \%(p=0.2)$. 
Table 6.1 Mean pharmacokinetic parameters for Tac BID and Tac QD.

\begin{tabular}{|c|c|c|c|}
\hline Parameter & $\begin{array}{c}\text { Tac BID } \\
\mathrm{N}=40 \\
(90 \% \mathrm{Cl})\end{array}$ & $\begin{array}{c}\text { Tac QD } \\
\mathrm{N}=40 \\
(90 \% \mathrm{Cl})\end{array}$ & $p$ \\
\hline Daily dose $(\mathrm{mg} / \mathrm{kg})$ & $\begin{array}{c}0.05 \\
(0.04 ; 0.02-0.12)^{a}\end{array}$ & $\begin{array}{c}0.05 \\
(0.04 ; 0.02-0.13)^{a}\end{array}$ & 0.09 \\
\hline $\mathrm{AUC}_{0-24}(\mu \mathrm{g} \cdot \mathrm{h} / \mathrm{l})$ & $\begin{array}{c}219.2 \\
(208.1-230.9)\end{array}$ & $\begin{array}{c}213.3 \\
(202.6-224.5)\end{array}$ & 0.37 \\
\hline $\operatorname{DnAUC}_{0-24}(\mu \mathrm{g} \cdot \mathrm{h} / \mathrm{l} / \mathrm{mg} / \mathrm{kg})$ & $\begin{array}{c}4944 \\
(4358-5414)\end{array}$ & $\begin{array}{c}4793 \\
(4244-5414)\end{array}$ & 0.30 \\
\hline$C_{\min }(\mu \mathrm{g} / \mathrm{l})$ & $\begin{array}{c}7.4 \\
(7.0-7.7)\end{array}$ & $\begin{array}{c}6.6 \\
(6.2-7.0)\end{array}$ & 0.003 \\
\hline $\mathrm{DnC}_{\min }(\mu \mathrm{g} / \mathrm{l} / \mathrm{mg} / \mathrm{kg})$ & $\begin{array}{c}166.1 \\
(144.9-190.4)\end{array}$ & $\begin{array}{c}146.2 \\
(127.7-167.3)\end{array}$ & $<0.001$ \\
\hline $\begin{array}{l}\text { Intraindividual variability } \\
\text { AUC (\%) }\end{array}$ & $\begin{array}{c}14.1 \\
(12.3-16.0)\end{array}$ & $\begin{array}{c}10.9 \\
(9.4-12.4)\end{array}$ & 0.012 \\
\hline $\begin{array}{l}\text { Intraindividual variability } \\
\mathrm{C}_{\min }(\%)\end{array}$ & $\begin{array}{c}15.3 \\
(13.3-17.3) \\
\end{array}$ & $\begin{array}{c}13.7 \\
(12.2-15.2) \\
\end{array}$ & 0.21 \\
\hline
\end{tabular}

Paired samples t-test was used for statistical analysis. Values are mean ( $90 \%$ confidence interval) unless indicated otherwise. ${ }^{a}=$ mean (median; range).

\section{Cytochrome P450 3A5 polymorphisms}

Table 6.2 and Figure 6.4 depict the influence of the cytochrome P450 3A5 polymorphism on the intrapatient variability during Tac BID and Tac QD. In the Cyp3A5 *1/*3 group, the CV decreased from $18.2 \%$ to $12.8 \%(P=0.06)$, while in the Cyp3A $5 * 3 / * 3$ group the CV decreased from $12.6 \%$ to $10.2 \%$ ( $p=0.16)$.

The statistical significant difference in CV between both CYP3A5 groups during Tac BID became numerically much smaller after conversion and was not statistically significant anymore $(p=0.22)$. Changes in $C V$ for $C_{\min }$ were in the same direction, although to a lesser extent.

Table 6.2 Mean intrapatient variability (CV) according to Cyp3A5 genotype.

\begin{tabular}{|c|c|c|c|c|c|c|}
\hline \multirow[t]{2}{*}{ Parameter } & \multicolumn{3}{|c|}{ Сур3A5*3/*3 $(\mathrm{N}=29)$} & \multicolumn{3}{|c|}{ Сур3A5*1/*3 (N=11) } \\
\hline & $\begin{array}{l}\text { Tac BID } \\
(90 \% \mathrm{Cl})\end{array}$ & $\begin{array}{c}\text { Tac QD } \\
(90 \% \mathrm{Cl})\end{array}$ & $p^{a}$ & $\begin{array}{c}\text { Tac BID } \\
(90 \% \mathrm{Cl})\end{array}$ & $\begin{array}{c}\text { Tac QD } \\
(90 \% \mathrm{Cl})\end{array}$ & $p^{a}$ \\
\hline Daily dose & $0.04^{b}$ & $0.04^{c}$ & 0.317 & $0.07^{b}$ & $0.08^{\mathrm{c}}$ & 0.180 \\
\hline$(\mathrm{mg} / \mathrm{kg})$ & $\begin{array}{c}(0.03 \\
0.02-0.11)^{d}\end{array}$ & $\begin{array}{c}(0.04 ; \\
0.02-0.11)^{d}\end{array}$ & & $\begin{array}{c}(0.07 ; \\
0.03-0.12)^{d}\end{array}$ & $\begin{array}{c}(0.07 ; \\
0.03-0.13)^{d}\end{array}$ & \\
\hline $\begin{array}{l}\text { CV AUC }{ }_{0-24} \\
(90 \% \mathrm{Cl})\end{array}$ & $\begin{array}{c}12.6^{\mathrm{e}} \\
(10.9-14.4)\end{array}$ & $\begin{array}{c}10.2^{f} \\
(8.5-12.0)\end{array}$ & 0.163 & $\begin{array}{c}18.2^{\mathrm{e}} \\
(13.5-22.9)\end{array}$ & $\begin{array}{c}12.8^{f} \\
(9.8-15.8)\end{array}$ & 0.062 \\
\hline $\begin{array}{l}\mathrm{CV} \mathrm{C}_{\min } \\
(90 \% \mathrm{Cl})\end{array}$ & $\begin{array}{c}14.6^{\mathrm{g}} \\
(12.6-16.5)\end{array}$ & $\begin{array}{c}13.2^{\mathrm{h}} \\
(11.6-14.8)\end{array}$ & 0.524 & $\begin{array}{c}17.2^{\mathrm{g}} \\
(11.5-23.0)\end{array}$ & $\begin{array}{c}15.0^{\mathrm{h}} \\
(11.2-18.8)\end{array}$ & 0.657 \\
\hline
\end{tabular}

Values are mean (90\% confidence interval), unless indicated otherwise. ${ }^{a}$ Wilcoxon signed ranking test.

${ }^{b} p=0.03 ;{ }^{c} p=0.03 ;{ }^{d}$ mean (median; range); ${ }^{e} p=0.05 ;{ }^{f} p=0.22 ;{ }^{g} p=0.55 ;{ }^{h} p=0.49$; all Mann Whitney $U$ test of Сур3A5*3 vs. Сур3A5*1 within each Tac formulation. 


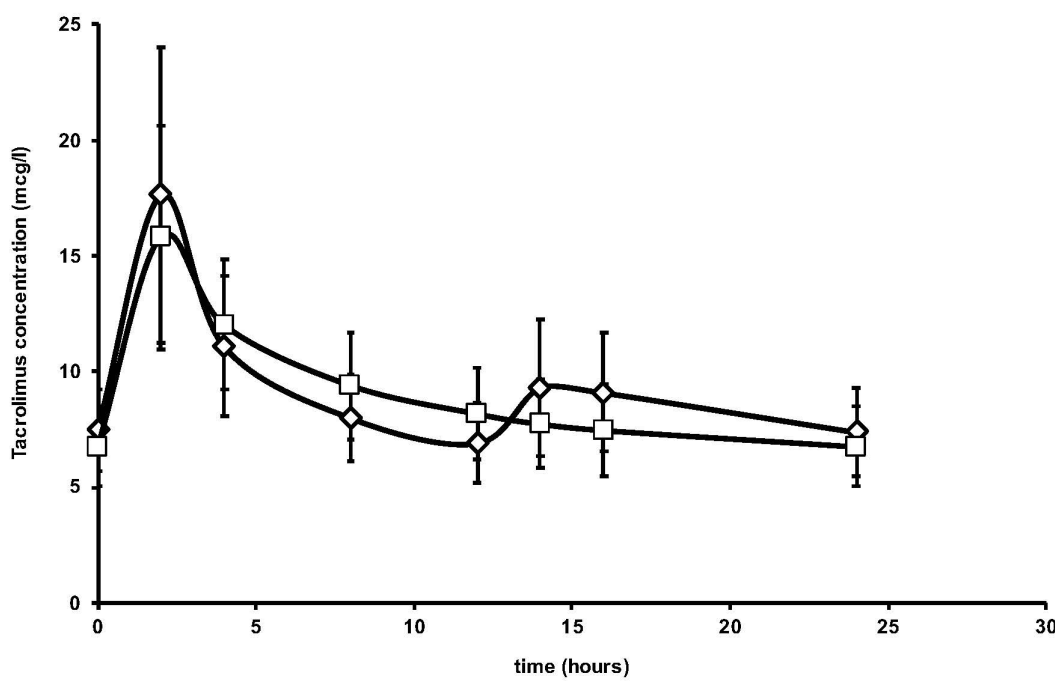

Figure 6.2 Mean PK profiles for Tac BID and Tac QD.

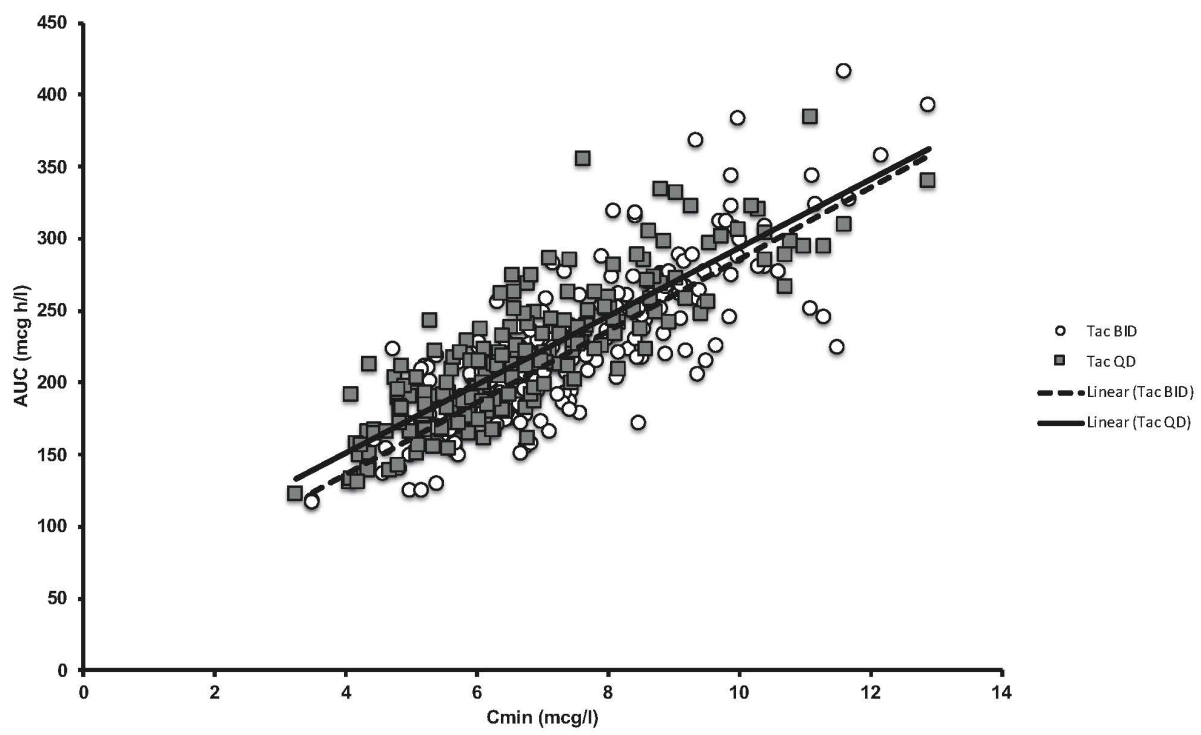

Figure 6.3 Correlation of $\mathrm{AUC}_{0-24}$ and $\mathrm{C}_{\min }$ for Tac BID and Tac QD. Regression equation for Tac BID: $y=24,938 x+36,618$. Regression equation for Tac $Q D: y=23,681 x+56,91$. 


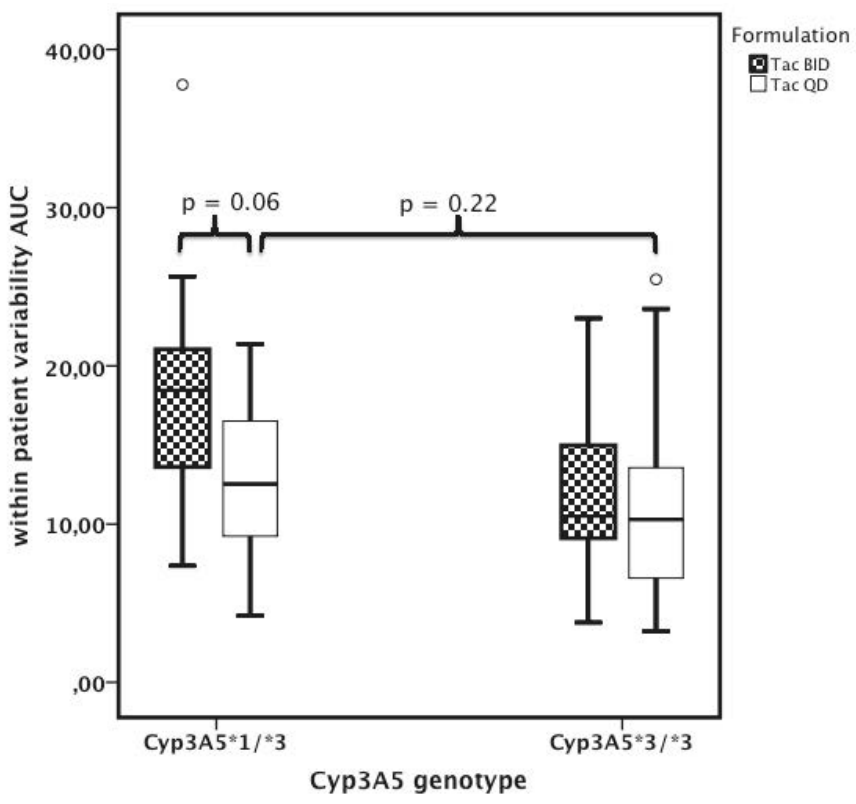

Figure 6.4 Blox plot of the intrapatient (CV) in AUC 0-24 for Tac BID and Tac QD according to the Cyp3A5 genotype. "o" denotes outlying value. Box delineates the first and third quartiles (interquartiles range [IQR]), the horizontal black line gives the median, and the whiskers give the highest and lowest values with 1.5 times the IQR.

\section{Discussion}

Our study is the first study that was designed to prospectively study the change in intrapatient variability in systemic tacrolimus exposure after converting from Tac BID to Tac QD in stable renal transplant patients. Five sequential PK profiles both during Tac BID and Tac QD were collected under steady state conditions by means of the DBS method. ${ }^{11}$ At the time of the study, this method had routinely been used for measuring Tac levels in our outpatient clinical practice for more than five years.

We have proven that this method is very reliable for determining Tac levels, especially when performed by well-instructed and motivated patients. ${ }^{11}$ The low percentage of missing crucial samples and samples of low quality in this study is another proof that the DBS method is robust. Only 12 out of the 400 profiles had to be repeated.

A significant improvement in the intrapatient variability in 24-hour exposure after converting from Tac BID to Tac QD was found, confirming previous post hoc analyses. ${ }^{1,2}$ This improvement of $\mathrm{CV}$ can be caused by either improved adherence of 
the patients or by intrinsic differences in the PK properties of the two Tac formulations. Although nonadherence can never completely be excluded, it is very unlikely that it has occurred with these selected patients and with this strict protocol. Hence, the improvement of intrapatient CV after converting from Tac BID to Tac QD is most likely due to differences in PK properties between Tac QD and Tac BID.

Although the AUC differed only by $-2.7 \%$ between Tac BID and Tac QD, $C_{\text {min }}$ did so by 10.8\%. Earlier phase 2 trials showed a 5-10\% lower AUC for Tac QD in stable kidney transplant recipients in comparison to $\mathrm{Tac} \mathrm{BID}^{1,2,12}$ with a larger patient population than in our study (60 and 67 vs. 40). It could be that our study was underpowered to detect a significant difference with respect to the AUC but values and the corresponding confidential interval of the current study comply with those earlier reports. Our current study was not designed to compare relative bioavailability of Tac BID and Tac QD. On the other hand, we also found an identical relationship between $C_{\min }$ and $A U C$ given the virtually identical slopes of the regression lines (Figure 6.3).

Especially in the Cyp3A5*1/*3 group, the intrapatient CV decreased after conversion, although this did not reach statistical significance $(\mathrm{P}=0.062)$. It has been hypothesized that Сур3A activities are generally decreased in the Cyp3A5*3/*3 genotype, and the chances of being influenced by environmental factors would then increase through induction of Cyp3A4 and Cyp3A5 by the Pregnane $X$ receptor (PXR). ${ }^{13}$ Cyp3A4 is postulated to be more prone to induction than Cyp3A5 and since, in Cyp3A5 expressors, the Cyp3A5 activity would be rather stable, different amounts of Cyp3A4 could cause substantial intra-individual variability.

On the contrary, Pashaee et al. could not establish a relationship between the Cyp3A5 genotype and the intrapatient variability. ${ }^{14}$ We found in our study that Cyp3A $5 * 1 /{ }^{*} 3$ carriers had a higher CV under Tac BID, which declined after converting to the oncedaily formulation. This would be in line with the findings of Alloway et al. and Wu et al. ${ }^{1,7}$, at least if we assume that the African-Americans and Chinese represent a high prevalence of the Cyp3A $5 * 1$ genotype. ${ }^{8,9}$

In our white population, we included only a limited number of patients with the Cyp3A5*1 allele and none of the patients were homozygous for this allele. Also in patients homozygous for the Cyp3A5*1 allele a larger decrease in CV can be expected. Nevertheless, in our study the Cyp $3 \mathrm{~A}^{*} 1 /{ }^{*} 3$ group had a significant higher intrapatient CV compared to the Cyp3A5*3/*3 group under Tac BID, a difference that was not significant anymore after converting to Tac QD.

In conclusion, we confirmed the reduced intrapatient CV for Tac QD compared with Tac BID. This improvement does not merely result from improved adherence but reflects intrinsic pharmacokinetic properties of Tac QD. The long-term clinical significance of a decreased variability in Tac drug exposure has to be determined yet. Thereafter, patients might profit from a drug with a reduced variability in systemic exposure. The reduction in intrapatient CV is numerically larger in patients with the Cyp3A5*1 allele. A limitation of our study is the relatively small number of patients 
carrying the Cyp3A5*1 allele and especially the absence of Cyp3A5*1 homozygotes. This should be explored in a future study.

\section{Acknowledgements}

We would like to thank our research nurse, Mrs Monique Mullens, for her support during the study. The study would not have been feasible otherwise. 


\section{References}

1. Alloway R, Steinberg S, Khalil K, et al. Conversion of stable kidney transplant recipients from a twice daily Prograf-based regimen to a once daily modified release tacrolimus-based regimen. Transplant Proc 2005;37(2):867-870.

2. van Hooff J, Van der Walt I, Kallmeyer J, et al. Pharmacokinetics in stable kidney transplant recipients after conversion from twice-daily to once-daily tacrolimus formulations. Ther Drug Monit 2012;34 (1): 46-52.

3. Budde K, Glander P. Pharmacokinetic principles of immunosuppressive drugs. Ann Transplant 2008; $13(3): 5-10$.

4. Florman S, Alloway R, Kalayoglu M, et al. Conversion of stable liver transplant recipients from a twicedaily Prograf-based regimen to a once-daily modified release tacrolimus-based regimen. Transplant Proc 2005;37(2):1211-1213.

5. Borra LC, Roodnat JI, Kal JA, Mathot RA, Weimar W, van Gelder T. High within-patient variability in the clearance of tacrolimus is a risk factor for poor long-term outcome after kidney transplantation. Nephrol Dial Transplant 2010;25(8):2757-2763.

6. Kuypers DR, Peeters PC, Sennesael JJ, et al. Improved adherence to tacrolimus once-daily formulation in renal recipients: a randomized controlled trial using electronic monitoring. Transplantation 2013; 95(2):333-340.

7. $\mathrm{Wu} \mathrm{MJ}$, Cheng $\mathrm{CY}, \mathrm{Chen} \mathrm{CH}$, et al. Lower variability of tacrolimus trough concentration after conversion from prograf to advagraf in stable kidney transplant recipients. Transplantation 2011;92 (6):648-652.

8. Cheung $\mathrm{CY}$, Op den Buijsch RA, Wong KM, et al. Influence of different allelic variants of the CYP3A and $A B C B 1$ genes on the tacrolimus pharmacokinetic profile of Chinese renal transplant recipients. Pharmacogenomics 2006;7(4):563-574.

9. Lee SJ, Usmani KA, Chanas B, et al. Genetic findings and functional studies of human CYP3A5 single nucleotide polymorphisms in different ethnic groups. Pharmacogenetics 2003;13(8):461-472.

10. van Schaik RH, van der Heiden IP, van den Anker JN, Lindemans J. CYP3A5 variant allele frequencies in Dutch whites. Clin Chem 2002;48(10):1668-1671.

11. Hoogtanders K, van der Heijden J, Christiaans M, Edelbroek P, van Hooff JP, Stolk LM. Therapeutic drug monitoring of tacrolimus with the dried blood spot method. J Pharm Biomed Anal 2007;44(3): 658-664.

12. Wlodarczyk Z, Squifflet JP, Ostrowski M, et al. Pharmacokinetics for once- versus twice-daily tacrolimus formulations in de novo kidney transplantation: a randomized, open-label trial. Am J Transplant 2009;9(11):2505-2513.

13. Yong Chung J, Jung Lee Y, Bok Jang S, Ahyoung Lim L, Soo Park M, Hwan Kim K. CYP3A5*3 genotype associated with intrasubject pharmacokinetic variation toward tacrolimus in bioequivalence study. Ther Drug Monit 2010;32(1):67-72.

14. Pashaee N, Bouamar R, Hesselink DA, et al. CYP3A5 genotype is not related to the intrapatient variability of tacrolimus clearance. Ther Drug Monit 2011; 33 (3): 369-371.

15. Op den Buijsch RA, van de Plas A, Stolk LM, et al. Evaluation of limited sampling strategies for tacrolimus. Eur J Clin Pharmacol 2007;63(11):1039-1044.

16. Cheung $\mathrm{CY}$, van der Heijden J, Hoogtanders K, et al. Dried blood spot measurement: application in tacrolimus monitoring using limited sampling strategy and abbreviated AUC estimation. Transpl Int 2008;21(2):140-145.

17. Hoogtanders K, van der Heijden J, Christiaans M, van de Plas A, van Hooff J, Stolk L. Dried blood spot measurement of tacrolimus is promising for patient monitoring. Transplantation 2007;83(2):237-238.

18. Note for Guidance on the Investigation of Bioavailability and Bioequivalence (NfG on BA BE). European Medicines Agency, ed. London, 2010. 


\section{Chapter 7}

General discussion and summary 



\section{Introduction}

Kidney transplantation is the leading modality for renal replacement therapy in endstage kidney disease. In 2018, 998 renal transplantations were performed in the Netherlands ${ }^{1}$, and 11405 patients were alive with a functioning transplant, whereas 6252 patients with end-stage kidney disease were on dialysis (data retrieved from: https://www.nefrovisie.nl/nefrodata/). Since induction of immunological tolerance of the kidney allograft is still not feasible, immunosuppression to prevent allograft rejection will remain the major component of post-transplant medical therapy. ${ }^{2}$ Currently, immunosuppressive drug regimens are mainly tacrolimus-based. ${ }^{3}$ Tacrolimus is often combined with other immunosuppressants like mycophenolate mofetil/mycophenolic acid, mTOR-inhibitors (sirolimus or everolimus) and steroids (prednisolone). The intensity of the immunosuppressive regimen may differ between transplantation centres, depending on the local protocol. The transplantation policy at the Maastricht University Medical Centre, for example, has been marked by early steroid withdrawal and $70 \%$ of the patients have tacrolimus monotherapy. ${ }^{4}$

Tacrolimus has been available since 1993 as "Prograf ${ }^{\circledR}$ (in the Netherlands "Prograft ${ }^{\circledR}$ "). The tacrolimus capsules are usually taken orally twice daily (BID). Since 2007, a modified release once-daily (QD) formulation of tacrolimus (registered under the trade name $\operatorname{Advagraf}^{\oplus}$ ) has become available with a similar efficacy and safety profile compared to the BID formulation. ${ }^{5}$ In this thesis, Tac BID refers to Prograf ${ }^{\circledR}$ and Tac QD refers to Advagraf ${ }^{\oplus}$. Also, an intravenous (i.v.) solution is available for cases in which normal oral administration is not feasible, however, in clinical practice, i.v. application comes with pitfalls. ${ }^{6}$

Since the mid 90's short-term graft survival has improved to over $90 \%$ at one year., ${ }^{7,8}$ Despite the improvement of short-term graft survival in this era, initially long term graft survival had not improved as much. ${ }^{9-11}$ However, more recently, long-term graft survival has improved as well, even more than in the short-term survival. ${ }^{9}$ These improvements were given despite worse donor and recipient characteristics, and might reflect advances in global kidney transplant management over the past decades. $^{9}$

Tacrolimus is a so-called 'Narrow Therapeutic Index Drug' (NTI) as reviewed extensively by Staatz et al. ${ }^{12}$, i.e. there is a small difference between underdosing (related to more rejection) and overdosing (related to more toxicity). Tacrolimus levels below $<5 \mu \mathrm{g} / \mathrm{l}$ are associated with antibody-mediated rejection (ABMR). ${ }^{13-15}$ In addition, there are also marked individual differences in absorption and metabolism which may also change during follow-up ${ }^{16}$, resulting in substantial inter- and intraindividual variability of tacrolimus levels. ${ }^{12}$ The latter is an established risk factor for later graft loss. ${ }^{17-23}$ 
Variability of tacrolimus levels is multifactorial. These factors can be patient- related but also drug-related. Some patient related factors may not be modifiable, like gender, Cyp3A5 genotype, age, albumin, or haematocrit. Other factors, however, may very well be modifiable, e.g. co-medication, ingestion of food and, above all, therapy adherence. Modifiable drug-related factors mainly consider its pharmacokinetic behaviour. These observations in the field of kidney transplantation implicate the urge for optimal tacrolimus-based therapy guided by therapeutic drug monitoring (TDM). Given the relevance for long-term graft survival, strategies to lower tacrolimus variability are extremely relevant, as are tools to identify the patients at risk. Altogether, this should result in stable and safe drug exposure.

This thesis tried to contribute to this aim by delivering new insights for the optimization of tacrolimus therapy. It focused on several pharmacokinetic and pharmacogenetic aspects of tacrolimus-based therapy in renal transplantation from a clinically relevant viewpoint. On the one hand, we investigated pharmacokinetic aspects referring to the Tac BID formulation (Part I: Chapters 2 and 3). Conclusions from these studies are probably valid for the substance tacrolimus, as such. On the other hand, pharmacokinetic studies on the newer Tac QD formulation were performed (Part II: Chapters 4-6).

\section{Part I}

As introduced above, the transplantation policy at the Maastricht University Medical Centre has been marked by early steroid withdrawal. It had been shown that stopping steroids after 10 days gave the same incidence of acute rejection as tapering after three months. ${ }^{24}$ Since then, steroids are preferentially withdrawn at day 10 after an uncomplicated transplantation in patients with a low immunological risk. It has been observed that after steroid tapering and withdrawal, dose-normalized tacrolimus trough levels $\left(\mathrm{DnC}_{0}\right)$ rise. ${ }^{25}$ In Chapter 2, the role of genetic polymorphisms (SNP's) of the Pregnane $X$ Receptor (PXR) and Cyp3A5 in this observation have been investigated.

In Chapter 3, sublingual and rectal routes of tacrolimus application were studied for potential circumstances that oral intake of the drug is not feasible. This study was based on reports that described therapeutic tacrolimus levels after sublingual application. ${ }^{26}$ These observations could not be confirmed in an earlier pilot study performed at our centre. ${ }^{27}$ However, significant tacrolimus levels after rectal application had been found. ${ }^{27}$ Firm pharmacokinetic data on sublingual and rectal application were lacking. This was studied as described in Chapter 3. 


\section{Part II}

In Chapters 4, 5 and 6 pharmacokinetic aspects of the newer once daily modified release tacrolimus formulation (Tac QD = Advagraf) are studied. In Chapter 4, Limited Sampling Strategies (LSS's) for the prediction of the 24-hour tacrolimus exposure $\left(\mathrm{AUC}_{0-24}\right)$ have been developed and validated.

In Chapter 5, the influence of a standard continental breakfast on the pharmacokinetics of Tac QD was studied in stable renal transplant patients. In healthy volunteers, a decreased absorption of Tac QD had been described when Tac QD was taken together with breakfast. ${ }^{5}$ However, these studies have not been performed in renal transplant recipients.

Finally, in Chapter 6, the intrapatient variability (IPV) was studied in stable renal transplant patients after converting Tac BID to Tac QD. There were no data available about the change in IPV of the $\mathrm{AUC}_{0-24}$ after converting to Tac QD, so far. This study was particularly important because a high IPV is related to decreased long-term graft survival $^{17-23}$, as already mentioned above.

\subsection{Steroids, Cyp3A and Pregnane X Receptor}

Since the introduction of tacrolimus, renal transplant recipients from the Maastricht University Medical Centre have been withdrawn from steroids progressively faster, Especially since the observation that stopping steroids after 10 days gave the same incidence of acute rejection as tapering after three months ${ }^{24}$, steroids are withdrawn at day ten after an uncomplicated transplantation. After steroid withdrawal, we and others have observed that tacrolimus dose requirements diminish in most, but not in all patients. ${ }^{16,25,28,29}$ The inter-individual difference in response to steroid tapering or withdrawal shows a wide scatter ${ }^{25}$ and it is unknown which factors determine this. Since steroids are reported to induce the activity of Cytochrome P450 3A (Cyp3A) enzymes ${ }^{30}$, it has been postulated that steroid tapering may result in diminished Сyp3A5 activity $^{25}$, resulting in decreased tacrolimus requirements.

Another candidate is the Pregnane $X$ Receptor (PXR, also called NR1/2: nuclear receptor 112). It is a regulatory factor of the Nuclear Receptor family with a steroid receptor and is involved in the upregulation of many drug-metabolizing enzymes and drug transporters ${ }^{31,32}$, amongst others, the Cyp3A. ${ }^{33}$ As the PXR is implicated in the transcriptional regulation of the Cyp3A enzymes, SNP's of the PXR may also influence tacrolimus metabolism during steroid taper. The PXR SNP's that have been associated with a higher magnitude of (intestinal) Cyp3A inducibility are the A7635G and the C8055T, while the C25385T SNP has been reported to be related to differences in tacrolimus apparent clearance. ${ }^{30,34}$ A change in tacrolimus exposure after steroid taper or withdrawal may thus differ between single nucleotide polymorphisms (SNPS) 
of either the Сyp3A5 or the PXR, or both. The possible mechanistic interaction between steroids, PXR, Cyp3A5 and the Tacrolimus concentration is illustrated in Figure 7.1.

a)

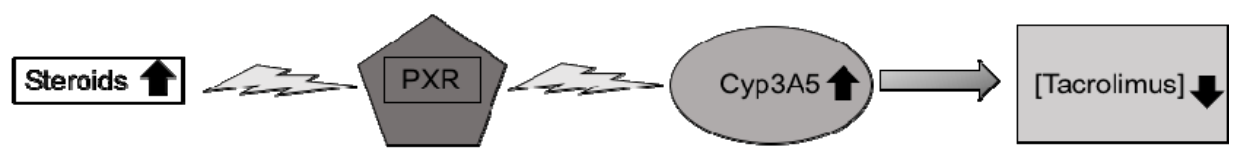

b)

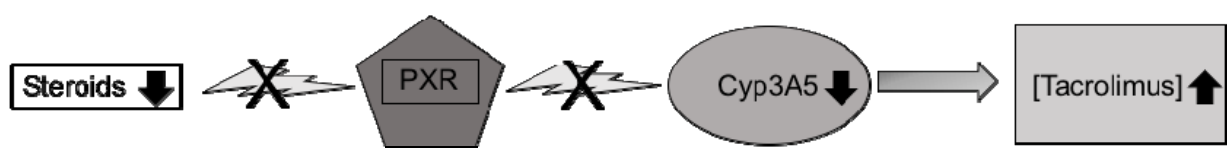

Figure 7.1 Interplay between steroids and tacrolimus via PXR and Cyp3A5. a) Steroids induce PXR that upregulates Cyp3A5 resulting in metabolization of tacrolimus. b) Lower or absent steroid dosing attenuates PXR and Cyp3A5 activity resulting in higher tacrolimus levels.

Therefore, in Chapter 2, it has been investigated whether the observed increase in tacrolimus exposure after steroid tapering ${ }^{16,25,29}$ could be mediated by SNP's of the PXR and Cyp3A5. In a cohort of 83 renal transplant recipients, the influence of PXR and Cyp3A5 SNP's and their potential interaction with each other on the Dosenormalized tacrolimus trough levels $\left(\mathrm{DnC}_{0}\right)$ was retrospectively analysed. The change in $\mathrm{DnC}_{0}$ was compared while the patients were on prednisone $10 \mathrm{mg}$ versus dose reduction from 10 to $5 \mathrm{mg}$ and after complete withdrawal. An (arbitrarily) 20\% change was considered to be clinically relevant,

Only 18 patients (21\%) remained stable with respect to the $\mathrm{DnC}_{0}$. About $75 \%$ of the individuals ( $n=62$ ), had an increase (up to $260 \%$ with a single outlier of $>400 \%$ ), while $5 \%(n=3)$ had a decline in $\mathrm{DnC}_{0}$ (up tot $\left.45 \%\right)$. This is comparable with an earlier report on the effect of steroid tapering on tacrolimus levels, where $61 \%$ of the patients had an increase in $\mathrm{DnC}_{0}$ of more than 20\% (up to 220\%) after withdrawal of $10 \mathrm{mg}$ prednisolone. ${ }^{25}$ In line with previous reports, carriers of the Cyp3A5*1 genotype had a lower $\mathrm{DnC}_{0}$ compared to the Cyp $3 \mathrm{~A} 5 * 3$-homozygotes ${ }^{35,36}$ both while on steroids (at baseline) and after steroid withdrawal . 
New findings of our study were:

- Carriers of Cyp3A5*1 exhibit a significant lower rise in $\mathrm{DnC}_{0}$ after steroid taper compared to the Cyp3A5*3 homozygotes (+33 vs. $+66 \%$ ).,

- PXR plays a role in tacrolimus pharmacokinetics: the slope of the change in $\mathrm{DnC}_{0}$ after steroid withdrawal is dependent on the PXR 7635A>G SNP. Compared to the homozygote PXR A7635A genotype, the A7635G heterozygotes had a trend towards a higher increase in $\mathrm{DnC}_{0}(+0.17 \mu \mathrm{g} / \mathrm{l} / \mathrm{mg}$ for every $5 \mathrm{mg}$ prednisolone tapering, $p=0.138$ ), while for the $G 7635 G$ homozygotes this increase was statistically and clinically significant and nearly twice as high $(+0.31 \mu \mathrm{g} / \mathrm{l} / \mathrm{mg}$, $\mathrm{p}=0.02)$.

- $\quad$ The PXR 7635A>G SNP and the Cyp3A5 SNP have independent correlations with the change in $\mathrm{DnC}_{0}$ after steroid withdrawal.

The rise in $\mathrm{DnC}_{0}$ has been documented in $75 \%$ of all patients, $25 \%$ of the patients in our cohort were PXR G7635G homozygotes. This means that the observed rise in $\mathrm{DnC}_{0}$ during steroid tapering is also present in patients without this SNP and thus only partially explains the inter-individual spread in response to steroid withdrawal as observed in our study. Still, the genotype frequency in our study population of this SNP is substantially higher than that for the Cyp3A5*1 SNP in a Caucasian kidney transplant recipient population, so there could be a clinical relevance. Due to the change of tacrolimus pharmacokinetics after steroid tapering or withdrawal, steroid dose reduction should be accompanied by therapeutic drug monitoring. On the other hand, when steroids are prescribed, increased tacrolimus requirements should be considered, and levels should be monitored.

The use of steroids in transplantation has been the subject of a longstanding debate. ${ }^{37}$ There is strong evidence that steroid withdrawal is advantageous in reducing cardiovascular risk factors (hyperlipidemia, hypertension and diabetes mellitus). ${ }^{38}$ There have been concerns about the short and long-term risk for the graft. The 10 -years patient survival has been reported to be higher in a large observational single-centre study, where steroids had been withdrawn in stable kidney allograft recipients with a low immunological risk. ${ }^{39}$ It has been observed in a very large randomized multicenter trial that stopping steroids after three months from tacrolimus, mycophenolate mofetil and steroids only resulted in a slight increased incidence in steroid-sensitive acute rejections. ${ }^{40}$ More recently, in a Cochrane review, no differences in patient mortality or graft loss up to five years after transplantation were found. ${ }^{37}$

In conclusion, besides the aforementioned reduction of hyperlipemia, hypertension and diabetes mellitus, the reduced tacrolimus requirements could considerably lower costs. Given the new insights that the PXR 7635A>G SNP is correlated to the change in $\mathrm{DnC}_{0}$, the clinician could now understand why patients show different patterns of tacrolimus dose requirement after steroid withdrawal. Theoretically, in which way and 
to which extent, respectively, SNP'S of the PXR and the Cyp3A5 might influence the changes in the $\mathrm{DnC}_{0}$. by steroid dose reduction is illustrated in Figure 7.2.

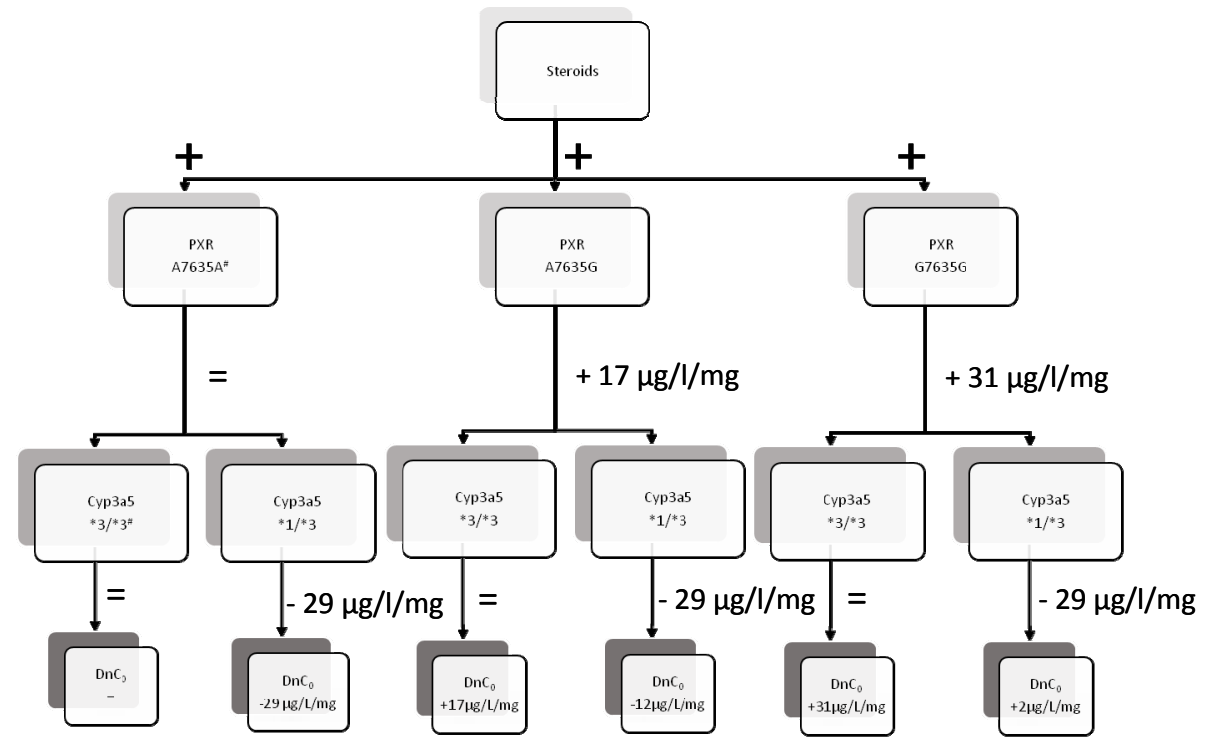

Figure 7.2 Schematic representation how SNP's of PXR and Cyp3A5 influence the $\mathrm{DnC}_{0}$. Steroids activate the PXR ("+"). Compared with the PXR A7635A wildtype ( ${ }^{\#}=$ Reference), per $5 \mathrm{mg}$ of steroid dose reduction, $\mathrm{DnC}_{0}$ will increase an additional 17 and $31 \mu \mathrm{g} / \mathrm{l} / \mathrm{mg}$ in PXR A7634G heterozygous and PXR G7635G homozygous patients, respectively. On the Cyp3A5 level, Cyp3A5*1-carriers have a $29 \mu \mathrm{g} / \mathrm{l} / \mathrm{mg}$ lower increase in $\mathrm{DnC}_{0}$ compared with Cyp3A5*3/*3 homozygous patients. The boxes in lowest line report the overall magnitude of the change in $\mathrm{DnC}_{0}$.

\subsection{Alternative formulations}

Normal gastro-intestinal absorption may be impaired under clinical circumstances (e.g. vomiting) or in cases that the normal swallowing act is not feasible (e.g. in intubated patients). These circumstances could threaten allograft survival. An intravenous solution is available, which comes with major adverse events owing to inappropriate dosages and adverse events of the infusion solution. ${ }^{6,41}$ Therefore, it has been suggested that drug absorption could be achieved through alternative sites such as the sublingual ${ }^{17}$ or the rectal route. ${ }^{42}$ However, for the sublingual route we were unable to confirm this in a pilot study. ${ }^{26,43}$ Extensive pharmacokinetic data on both sublingual and rectal tacrolimus absorption are lacking. Therefore, in Chapter $3, \mathrm{a}$ pharmacokinetic study of single dose sublingual and rectal formulations of tacrolimus 
in healthy volunteers has been performed. In this study, the pharmacokinetics of these formulations compared to the oral Tac BID formulation has been investigated.

\subsubsection{Sublingual application}

The powder obtained from the $3 \mathrm{mg}$ (i.e. $0.04 \mathrm{mg} / \mathrm{kg}$ ) Tac BID capsule was applied under the tongue for a period of 15 minutes without swallowing and subsequent rinsing of the mouth. Similar to the results our pilot study ${ }^{43}$, sublingual application failed to show consistent therapeutic tacrolimus blood concentrations: 11 out of 18 subjects did not have any concentrations above $1 \mu \mathrm{g} / \mathrm{l}$, i.e. below the lower limit of quantification. In 7 subjects tacrolimus concentrations were detected. In 3 of them a swallowing act was documented $\left(C_{\max }\right.$ ranging between 1.3 and $22 \mu \mathrm{g} / \mathrm{l}$ and a $T_{\max }$ of 2 hours), while in $4 \mathrm{C}_{\max }$ ranged from 1.3 to $7.2 \mu \mathrm{g} / \mathrm{l}$, also with a $\mathrm{T}_{\max }$ of 2 hours, without a documented swallowing act.

Our observations are in clear contrast with other reports that have described higher tacrolimus levels after sublingual administration..$^{26,44,45}$ We conclude that the systemic exposure described elsewhere probably resulted not from sublingual, but from regular lower gastrointestinal absorption. Our arguments for this conclusion are the following:

- $\quad A T_{\max }$ of 2 hours in the subjects that had swallowed is comparable with the $T_{\max }$ after oral intake of tacrolimus. ${ }^{46,47}$ The same $T_{\max }$ was found in previous reports that claimed therapeutic drug levels after sublingual application. 6,26,45 True sublingual absorption should come with a significantly shorter $\mathrm{T}_{\max .}{ }^{48}$ As in our study the tacrolimus powder was kept under the tongue for 15 minutes with mouth rinsing immediately afterwards, no delayed absorption could be expected. Therefore, the tacrolimus blood concentration-time profiles in reports that showed therapeutic drug levels are compatible with normal intestinal rather than with sublingual absorption. ${ }^{6,26,45}$

- Even in this prospective study with motivated healthy volunteers, it was nearly impossible to keep the powder content in their mouth without swallowing for the required 15 minutes, as stated by the volunteers and observed in several of them.

- A comment on our pilot study, was that the amount of powder applied was probably too low. ${ }^{6}$ However, it is our experience, that it is not possible to use a content larger than that of the $3 \mathrm{mg}$ capsule under the tongue for the required time without eliciting a swallowing act. Therefore, we assume unintended swallowing in other reports. Moreover, another study claimed detectable systemic tacrolimus levels with a similar amount of sublingual powder as we used. $^{49}$

- The studies that claimed systemic exposure after sublingual application were either retrospective ${ }^{6}$ or longitudinal clinical studies. ${ }^{26,44,45,49}$ Precautions to prevent swallowing have not been reported in the prospective studies. It is 
doubtable (and not mentioned) if this prohibition of swallowing could be successfully applied in the retrospective study. ${ }^{6}$ Under experimentally controlled circumstances, so far, no true consistent sublingual absorption after single dose application of the tacrolimus capsule powder content has been shown in healthy volunteers.

For these reasons, there is most probably no true sublingual absorption of tacrolimus powder. This way, feasibility of normal gastro-intestinal absorption should be considered during hospitalization of the renal transplant patient, instead of putting the patient at risk of the caveats associated with the i.v. solution. Contrary to the sublingual route, the rectal route has been found to be a potential site of tacrolimus application as reported in the next section.

\subsubsection{Rectal administration}

In Chapter 3, single dose rectal tacrolimus application via a suppository was studied in 18 healthy volunteers. This was the first PK study on rectal tacrolimus application, being adequately powered according to current EMEA guidelines. ${ }^{50}$ Compared to $5 \mathrm{mg}$ of the oral formulation, $15 \mathrm{mg}$ rectal tacrolimus had a lower dose-corrected $\mathrm{C}_{\max }$ $(1.5 \mu \mathrm{g} / \mathrm{l} / \mathrm{mg}$ vs. $4.9 \mu \mathrm{g} / \mathrm{l} / \mathrm{mg}$, geom. mean ratio 0.31 (Cl $0.27-0.37))$. It displayed a prolonged $\mathrm{T}_{\text {max }}$ : 4.2 hours ( $\mathrm{Cl} 3.3-5.1$ hours) vs. 1.58 hours ( $\mathrm{Cl} 1.4-1.80$ hours). The dose-corrected $\mathrm{AUC}_{0-24}$, was $30 \%$ lower for the rectal formulation: $19 \mathrm{vs.} 28 \mu \mathrm{g} \cdot \mathrm{h} / \mathrm{l} / \mathrm{mg}$; with a wide variation (14-43\% reduction). The elimination half-life was similar for the two formulations. The rectal formulation was more variable with an almost twice as high inter-subject variability compared to the oral formulation.

Intriguingly, despite the lower relative bioavailability all subjects had a relevant systemic tacrolimus exposure with the suppository with an average $A \mathrm{C}_{0-24}$ of $292 \mu \mathrm{g} \cdot \mathrm{h} / \mathrm{l}$ compared to $194 \mu \mathrm{g} \cdot \mathrm{h} / \mathrm{I}$ after oral intake. After 24 hours, the average tacrolimus concentration after oral administration was $3.32 \mu \mathrm{g} / \mathrm{l}$, whilst the concentration after rectal application was more than twice as high at that time point $(7.88 \mu \mathrm{g} / \mathrm{l})$, and with detectable concentrations even after 48 hours $(3.7 \mu \mathrm{g} / \mathrm{l})$. All these findings from our study imply several clinical practical considerations, which will be discussed below.

- First, rectal tacrolimus application might be an alternative route for adequate systemic tacrolimus exposure (e.g. in solid organ transplant recipients), indeed. However, higher doses have to be applied given the lower relative bioavailability.

- Second, rectal administration of tacrolimus not only provides therapeutic tacrolimus levels but systemic (side-)effects should also be considered.

- Third, rectal application comes with more variable drug exposure compared to oral application of the drug. 
So, when tacrolimus is given rectally, as is also done with the goal to induce a local therapeutic effect in patients suffering from colitis ${ }^{51}$, adequate therapeutic drug monitoring and screening for toxicity of tacrolimus is warranted.

Why rectal application of tacrolimus showed a lower bioavailability remains speculative, so far. In chapter 3 this has not been attributed to its amount or the compartmental size, but was hypothesized to be caused by other mechanisms like pre-systemic metabolism of tacrolimus mediated by mucosal enzymes, for example the Cyp3A5 and the P-glycoprotein (P-gp). ${ }^{52}$

The later $T_{\max }$ and the sustained detection of tacrolimus even after 48 hours indicate a prolonged absorption phase. This may also be related to the P-gp, since P-gp mRNA levels are reported to increase longitudinally along the intestine, with highest level in the colon. ${ }^{53,54}$ The Cyp3A5, on the other hand, is mainly localized in the proximal digestive tract $^{52,54,55}$ with decreasing catalytic activity downstream. ${ }^{56}$ Therefore, mucosal saturation and sustained mucosal P-gp-mediated drug efflux may be responsible for the prolonged absorption phase leading to sustained transport over the mucosal barrier and slow release of tacrolimus from the rectal mucosal cells into the circulation. However, the potential role of P-gp in the bioavailability and the absorption pattern was not investigated in our study. The generated questions need to be addressed in future studies.

Remarkably, eight of the 18 subjects depicted a biphasic absorption pattern (compared to a monophasic pattern in others) for reasons that are not completely understood. A possible explanation might be saturation of the mucosal transport, combined with insufficient delivery of tacrolimus molecules due to bad dissolution (absence of water in the rectum) ${ }^{47}$ and subsequent drug absorption in more proximal bowel segments. This remains speculative and this observation should also be confirmed in future studies.

Future studies should confirm our pharmacokinetic findings with repetitive ascending dosing (of labelled tacrolimus) to establish the optimal doses and dosing frequencies. This would also supply us with more data on dose-proportionality and on absorption patterns. These studies should also be performed in relevant patient populations (e.g. solid organ transplant recipients or patients with (distal) colitis).

In conclusion, if the oral route is unavailable, in contrast to sublingual application of the tacrolimus powder, rectal administration of a first-generation prototype suppository resulted in therapeutic drug exposure. In the clinical case of an absent oral route of administration, a regular suppository as could be manufactured in every hospital pharmacy, can deliver therapeutic tacrolimus blood concentrations. However, rectal tacrolimus is not routinely being used in transplant clinical practice yet and further data are lacking. For this reason, when tacrolimus will be given rectally, this 
should either be done under strict supervision of TDM expertise or in the context of scientific research.

\subsection{Therapeutic Drug Monitoring of Tac QD}

Since tacrolimus is an NTI-drug there is clear consensus that TDM has to be performed. The method, however, is a matter of debate. In a consensus report on Tac $B I D$, the Area under the blood concentration-time curve (AUC) from 0-12 hours $\left(A \cup C_{0-12}\right)$ has been denoted to be the best pharmacokinetic marker for Tac BID exposure. ${ }^{57}$ However, TDM by full AUC measurement is expensive, time consuming and inconvenient for patients and staff. Because the trough level showed good correlation with the $\mathrm{AUC}_{0-12} 57,58$, it is used as a surrogate marker for Tac BID exposure. However, several authors report only a moderate correlation and predictive value of the $C_{0}$ with the AUC for Tac BID. ${ }^{59-61}$ Limited sampling strategies (LSS's) consisting of two to three sampling points have shown to better predict AUC for Tac BID. ${ }^{62}$ For practical reasons, most of these sampling points are within the first hours post-dose.

Also for Tac QD formulation, a good correlation between trough level and 24 hour exposure $\left(A \cup C_{0-24}\right)$ has been reported. ${ }^{58}$ However, it is unknown whether other monitoring strategies allow more accurate prediction of the $\mathrm{AUC}_{0-24}$. Besides, LSS's developed to predict tacrolimus AUC for one formulation should not be considered to be valid for another formulation with a different absorption and elimination curve.

Therefore, in Chapter 4, LSS's for the prediction of $\mathrm{AUC}_{0-24}$ for Tac QD have been developed and validated in early and late post-transplant groups. The LSS's are based on linear regression analysis and its predictive performances have been compared with a population pharmacokinetic model (Maximum A Posteriori Bayesian estimation; MAPB).

It has been shown in our study that a linear regression-based LSS containing one single measurement during the elimination phase $\left(C_{8}\right)$ gives accurate estimation of $\mathrm{AUC}_{0-24}$ in an adult Caucasian stable renal transplant population. Sampling and estimation on the basis of MAPB confirmed the need for sampling in the elimination phase. None of the LSS's developed by linear regression analyses contained a trough value $\mathrm{C}_{0}$ or $\mathrm{C}_{24}$, unless it was forced into a model in combination with other sampling time points.

For de novo kidney transplant recipients, as from day 14 post-transplant, single time point measurement was insufficient. A three-point LSS most adequately predicted $\mathrm{AUC}_{0-24}$ : it contained a peak level at two hours post-dose $\left(\mathrm{C}_{2}\right)$, and two samples in the elimination phase at eight hours $\left(C_{8}\right)$ and at twelve hours $\left(C_{12}\right)$ post-dose. This means that accurate prediction in the early post-transplant phase, when the chances for acute rejection are high, estimation of the tacrolimus $\mathrm{AUC}_{0-24}$, is not guaranteed with 
trough level measurement only, but later and more frequent sampling should be considered. As long as the patients are hospitalized, this may be feasible. In an outpatient setting, later and more frequent venous sampling would be inconvenient. Capillary sampling, e.g. by a Dried Blood Spot (DBS), may facilitate this sampling strategy in an outpatient setting. ${ }^{63}$

\subsection{Tacrolimus-food interaction}

Both Tac BID and Tac QD are advised to be administered on an empty stomach, i.e. one hour before or two hours after a meal. This recommendation is based on reports that food decreases both the rate and the extent of absorption of tacrolimus with the effect being most pronounced after a high-fat meal. ${ }^{64,65}$ However, fasted intake of the drug is not convenient for the patients and may be a factor contributing to noncompliance.

Studies performed in our centre more than 20 years ago had demonstrated only a numerical, but not a significant change in Tac BID trough level in most, but not all patients when the drug was administered together with a continental breakfast. ${ }^{66-68}$ Therefore, in our centre, for reasons of convenience, patients have been allowed to convert to a non-fasted intake of the drug early post-transplant when their tacrolimus levels are stable. ${ }^{66}$

For Tac QD, fasted intake is also recommended on the basis of studies in healthy volunteers ${ }^{5,69}$, however, in transplant patients, no published data were available. Therefore, a prospective pharmacokinetic was conducted to assess the influence of a standard continental (low-fat) breakfast on the relative bioavailability of Tac QD in 27 (stable) kidney transplant recipients. (Chapter 5).

When Tac QD was ingested in a non-fasted state, i.e. together with a standard continental breakfast, the $\mathrm{AUC}_{0-24}$ showed an overall $14 \%$ decrease together with a prolongation of the $T_{\max }$ for one hour. Although the mean ratio of $\mathrm{AUC}_{0-24}$ is just below 0.9 , there was a large spread in individual changes in $\mathrm{AUC}_{0-24}$, ranging from -38 to $+29 \%$. In 16 out of 27 patients (59\%) the change of $A U_{0-24}$ was less than $20 \%$. Two patients (7\%) had an increase in $\mathrm{AUC}_{0-24}$ of $20 \%$ and $29 \%$, respectively. Nine patients (33\%) had a more than $20 \%$ decrease in $\mathrm{AUC}_{0-24}$ after ingestion of the drug together with the breakfast. Only in one patient the trough level fell below the lower limit of the target range $(<4 \mu \mathrm{g} / \mathrm{l})$, from 5.7 to $3.7 \mu \mathrm{g} / \mathrm{l}$.

Intriguingly, in patients with the Cyp3A5*1-genotype we observed only an $7 \%$ decrease in the $\mathrm{AUC}_{0-24}$ compared to the average $15 \%$ decrease in Cyp3A5*3/*3 homozygous patients. These results should be interpreted with precaution, since only five patients carried this genotype and no Cyp3A $5 * 1 /{ }^{*} 1$ homozygous patients could be included. 
Based on the results of our study, patients are allowed to take Tac QD together with a non-high fat continental breakfast, provided they have a stable renal function with adequate tacrolimus trough levels at about 2-3 weeks after transplantation, with trough level monitoring 7 days later. Future studies will have to prove if non-fasted intake of tacrolimus leads to better adherence. The observation of the weaker decrease in $\mathrm{AUC}_{0-24}$ in Cyp3A5*1-carriers could be of significance but needs confirmation in a larger Cyp3A $5 * 1$ population.

Given the inter-individual differences in the effect of food on tacrolimus exposure, we recommend monitoring of tacrolimus trough levels at one week after conversion to sustained non-fasted intake. Considering TDM, in this study, the relationship between the Tac QD trough level and the $\mathrm{AUC}_{0-24}$ is comparable in the therapeutic trough level range under fasted and non-fasted conditions. So as long as trough level measurement for TDM purposes is applied in an outpatient setting (with considerations as described in Chapter 4), trough level guidance of tacrolimus exposure can be used in clinical practice under these conditions.

\subsection{Intrapatient variability of Tac QD}

It is generally accepted that patients with a higher IPV (expressed as the coefficient of variation: (V) in Tacrolimus blood levels have disadvantageous long-term graft outcome. ${ }^{17-23,70}$ One factor contributing to fluctuating medication exposure considers differences between formulations. A drawback of conversion studies addressing IPV often is their retrospective design ${ }^{71}$, the use of trough levels as a surrogate marker for exposure $^{72-74}$ or only two measurements in case of calculation of the IPV of the $\mathrm{AUC}_{0-24}{ }^{58}$

For this reason, a prospective pharmacokinetic study was performed in 40 stable renal transplant patients with the purpose to investigate the change in IPV of systemic tacrolimus exposure after conversion from Tac BID to Tac QD (Chapter 6). For both formulations, five sequential 24-hour blood concentration-time profiles $\left(\mathrm{AUC}_{0-24}\right)$ consisting of 8 samples each were collected under steady state conditions. In addition, the potential influence of the Cyp3A5 carrier state was prospectively investigated.

After conversion from Tac BID to Tac QD, the IPV of the $\mathrm{AUC}_{0-24}$ improved statistically significant from $14.1 \%$ to $10.9 \%$ ( $22 \%$ relative reduction). The IPV was already low while on Tac BID as compared to other studies. ${ }^{20,71}$ Those studies are mostly observational. Since we investigated IPV prospectively, patients strictly had to follow the study protocol consisting of repetitive 24-hour AUC measurement (composed of eight blood samples each). For this reason, non-adherence can be assumed to be eliminated in our study, also supported by the fact that there were no missing blood concentration-time curves. Even with this strict adherence, we still found a significant 
reduction in $\mathrm{AUC}_{0-24}$ IPV after converting from Tac BID to Tac QD. This reduction in variability therefore reflect the mere differences in pharmacokinetic properties of the two tested formulations.

Due to the prospective nature of our conversion study with IPV measurement on the basis of repetitive 24-hour AUC measurement, direct comparison with other reports is impossible. Whereas we describe a reduced IPV in drug exposure by the gold standard $A \cup C_{0-24}$, other studies used the $C_{0}$ as a surrogate for drug exposure. ${ }^{74,75}$ In our study, the $\mathrm{CV}$ for $\mathrm{C}_{0}$ had also numerically declined (from 15.3 to $13.7 \%$ ) but was not statistically significant. As outlined in Chapter 4 (Limited Sampling strategies), we had found a worse correlation between the $\mathrm{C}_{0}$ and the $\mathrm{AUC}_{0-24}$. May be for this reason, the $\mathrm{C}_{0}$ is a less sensitive marker to detect changes in the IPV of true tacrolimus exposure and may explain the negative results of other reports with respect to changes in IPV, in line with Shuker et al.. ${ }^{75}$

A high IPV is particularly important in the lower range of tacrolimus levels with a higher risk of undertreatment and subsequent formation of $\operatorname{dnDSA}{ }^{76,77}$, histological lesions $^{78}$ and eventual graft loss. ${ }^{17-23}$ In earlier reports, a lower limit of the tacrolimus trough level of approximately $5 \mu \mathrm{g} / \mathrm{l}$ has been described, below which formation of dnDSA had been found. ${ }^{13,14}$ In a more recent paper, a tacrolimus trough level of $<4 \mu \mathrm{g} / \mathrm{l}$ at year 3 after transplantation has been associated with poorer graft survival in the two consecutive years. ${ }^{15}$ Also late after transplantation, a high CV has been found to be a strong predictor of kidney graft loss. ${ }^{15,21}$

In Cyp3A5*1-carriers, the CV for the $\mathrm{AUC}_{0-24}$ decreased even more from 18.2 to $12.8 \%$ (30\% relative reduction). The observed stronger improvement in IPV with switching to Tac QD in Cyp3A5*1-carriers has also been described after post-hoc analysis of a prospective trial in a Cyp3A $5 * 1$-enriched population ${ }^{79}$, but could not be confirmed in a recent retrospective study. ${ }^{80}$ These conflicting results should be clarified in prospective trials, since, if this influence was given, it suggests that Cyp3A5*1-carriers might potentially profit most from conversion to Tac QD in terms of reducing IPV. Nevertheless, conversion to TAC QD still contributes to minimize variability in drug exposure.

In conclusion, this study is so far the only prospective pharmacokinetic study on IPV during both Tac BID and Tac QD that has been able to collect data from repetitive 24-hour tacrolimus blood concentration-time profiles on each formulation and hereby delivering robust data on IPV in 24-hour drug exposure. With once daily dosing adherence to tacrolimus has been reported to improve ${ }^{81}$ and it intrinsically reduces pharmacokinetic variability of tacrolimus exposure compared with Tac BID as has been proven in Chapter 6 .

Given the association of worse graft survival in patients with a high IPV, tacrolimus therapy should not only consist of trough level measurement but the clinician should also be aware of the IPV. ${ }^{15}$ It has been suggested to be an important measure to 
identify patients "at risk" and may deliver a tool to monitor the success of implemented interventions. $^{82,83}$ In case of a high IPV, the clinician should look for potential causes. The search should certainly focus on therapy adherence but also on other influences. These might, amongst others, consider randomly switching between fasted or non-fasted intake of tacrolimus, the use of interacting co-medication or herbal supplements. How the IPV could be implemented in clinical practice and how to identify potential causes for a high IPV is presented in Figure 7.3 and Table 7.1, respectively. Home-based TDM (DBS) could facilitate the patient and the clinician in identifying these factors.

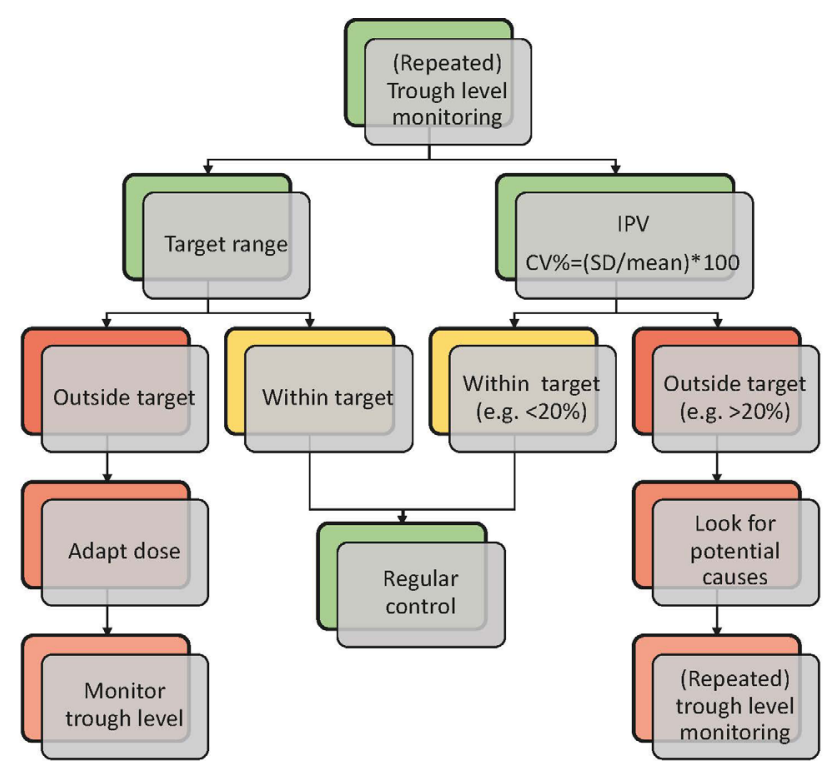

Figure 7.3 Flow chart for implementation of the IPV during post transplantation care. After repeated trough level measurement the clinician should not only consider whether or not the trough level is within the target range, but also the intra-patient variability (IPV) should be considered. The variation coefficient percentage (CV\%) is a measure for the IPV and calculated by dividing the standard deviation (SD) through the mean. Up until now, the cut-off value that qualifies a high IPV is unknown. Based on values used in literature, values from $16 \%$ or $22 \%$ have been used. ${ }^{22,82,83}$ In this flow chart a value in between (20\%) has arbitrarily been chosen. 
Table 7.1 Potential tools for identifying causes for high intra-patient variability of tacrolimus exposure.

\begin{tabular}{ll}
\hline Risk factor & Check for: \\
\hline Therapy adherence & Medication delivery (contact pharmacy) \\
& Correct dose \\
& Correct time \\
& Trough level (on demand: DBS) \\
& IPV \\
& Medication in use (contact pharmacy) \\
Drug or herbal interaction & Use of herbal products \\
& Trough level \\
Food intake & Random switch of fasted vs. non-fasted intake \\
& Time of drug intake in relation to food \\
& Fat content of food \\
Corticosteroids & Trough level \\
Generic substitution & Prescription or withdrawal of steroids \\
& Trough level \\
Tacrolimus assay & Medication in use (contact pharmacy) \\
& Trough level \\
Intercurrent illness & Contact laboratory: \\
& Different assay? \\
Quality control issues? & Diarrhea? \\
& Low haematocrit or albumin? \\
& Liver disease? \\
& Trough level \\
(Switch of) Time of ingestion & Trough level \\
\hline
\end{tabular}

\subsection{Conclusions, future directions and areas of uncertainty}

This thesis gives new insights in several aspects related to tacrolimus therapy. Discussed were the influence of polymorphisms of Cyp3A5 and PXR on the effect of steroid withdrawal, alternative routes of administration, adequate monitoring strategies, drug-food interaction and differences in IPV between Tac BID and Tac QD. The results presented in this thesis were derived from studies performed at our centre. Our kidney transplant population may differ from those in other centres. The majority of our patients is on tacrolimus monotherapy while in other centres, patients are mostly treated with more immunosuppressants (e.g. steroids, mycophenolate mofetil and/or mTor-inhibitors). It is unknown, whether this major difference in therapy influences some of the results presented in this thesis.

Considering sublingual application of the Tac BID powder, we have shown that tacrolimus will not be absorbed by the oral mucosa when the maximally tolerable quantity during the maximally tolerable duration is administered. Although repetitive 
or higher dosing might result in sublingual tacrolimus absorption, on the basis of our study, this seems rather unlikely. Improving oral mucosal absorption by incorporating a hydrophilic carrier for the lipophilic drug to increase the dissolution rate of tacrolimus ${ }^{84}$ has to be investigated in further studies.

For the rectal route of administration, we have demonstrated clinically relevant absorption of a single dose tacrolimus suppository, enabling systemic (side-) effects. Albeit the promising results of our study in healthy volunteers, studies in kidney transplant recipients are lacking up until now. First, further studies are needed to address the observed prolonged absorption, the causes and the role of the observed biphasic absorption pattern in part of the volunteers and the high inter-individual variability after rectal application. Second, there are no data on repetitive dosing and IPV for this formulation. Until then, whenever tacrolimus will be applied rectally, adequate TDM is mandatory.

Within the field of pharmacogenetics, differences in the rise of the $\mathrm{DnC}_{0}$ depending on Cyp3A5 and PXR polymorphisms have been demonstrated. As the genotype frequency of this SNP is substantially higher in our study population compared to that of the Cyp3A5*1 SNP in a Caucasian kidney transplant population, there could be a clinical relevance. As knowledge of the PXR genotype does not come with therapeutic consequences, so far, we do not advise to determine the PXR genotype pretransplantation, as is the case for the Cyp3A5 genotype. ${ }^{85}$ Although genotyping is not common practice in the field of transplant care, yet, research on pharmacogenetic testing and the potential introduction of a pharmacogenetic passport is currently being performed. ${ }^{86}$ Until then, awareness of the influences of the PXR SNP'S and the Cyp3A5 SNP's on tacrolimus pharmacokinetics could help the clinician to understand inter- and intra-individual differences in tacrolimus levels implying strict TDM.

Considering TDM, in this thesis, it has also been addressed that single sampling eight hours after dosing $\left(C_{8}\right)$ will better predict the Tac QD $A U C_{0-24}$ compared with $C_{0^{-}}$ sampling. It has also been shown that, especially early after transplantation, no single trough level-measurement sufficiently predicted $\mathrm{AUC}_{0-24}$, contrary to the three-point measurement of $C_{2}, C_{8}$ and $C_{12}$. Concerns considering the feasibility of more frequent or late blood sampling can be overcome by means of the dried blood spot sampling (DBS). ${ }^{63}$ However, there have been no studies that addressed how tight AUC-based TDM could result in better patient or graft outcome compared to $\mathrm{C}_{0}$-based TDM. ${ }^{87}$ Given the required time of follow-up with changing variability, it is unlikely that there will ever be a long- term prospective study on this issue.

Next, when Tac QD was ingested together with a non-high-fat (continental) breakfast, we found a mild decrease in drug exposure so that patients are allowed to convert to a non-fasted tacrolimus intake. Such a non-fasted intake should then be really consistent, i.e. without randomly switching between fasted and non-fasted intake. Also, the study has been restricted to taking the drug really together with the breakfast, i.e. not 15 minutes before or after. Conversion to non-fasted intake should 
always be followed by trough level monitoring given the large inter-individual spread in changes of the $\mathrm{AUC}_{0-24}$. It is currently unknown to what extent this implementation of non-fasted drug intake really results in improved therapy adherence. It has also been suggested in our study that patients with the Cyp3A5*1-genotype had a weaker influence of breakfast on tacrolimus exposure. This should be confirmed in a larger Cyp3A5*1 population.

Last, we performed the first clinical prospective study on the change in IPV in $\mathrm{AUC}_{0-24}$ after conversion from Tac BID to Tac QD and demonstrated an improved intrinsic IPV for Tac QD. Given the increased awareness of the relation between the IPV and the long-term graft outcome, our finding may help to minimize the IPV and its detrimental effects on graft survival. ${ }^{17-23}$ Still, medication non-adherence may mainly be responsible for tacrolimus variability. ${ }^{88}$ It is unknown how important the improved intrinsic IPV is for long term graft outcome, on top of improved therapy adherence. This will also probably be hard to prove, due to the required time of follow-up (similar to the question regarding TDM by AUC-guidance) and the high prevalence of Tac QD use in clinical transplant practice.

Whilst home-based TDM by means of the DBS has already been established in our outpatient practice ${ }^{63,89}$, the DBS-method can also be used for compilations of repetitive full 24-hour blood concentration-time profiles for the implementation in clinical research. The DBS allows for day to day trough level measurement or more extended LSS's for prediction of the $\mathrm{AUC}_{0-24}$. This delivers a tool to monitor the IPV for the sake of identifying patients at risk with subsequent intervention when necessary. This way, patients are empowered to take a blood sample at any desired time point while they are at home, contributing to home-based monitoring strategies and selfmanagement in the field of tailored transplantation care. 


\section{References}

1. Jaarverslag 2018 Nederlandse Transplantatie Stichting. Nederlandse Transplantatie Stichting. 2018.

2. Saidi R F, Hejazii Kenari SK. Clinical transplantation and tolerance: are we there yet? Int J Organ Transplant Med 2014;5(4): 137-145.

3. Staatz CE, Tett SE. Clinical Pharmacokinetics of Once-Daily Tacrolimus in Solid-Organ Transplant Patients. Clin Pharmacokinet 2015;54(10):993-1025.

4. van Hooff JG, van Duijnhoven E, van Heurn E; van den Berg-Loonen E, Christiaans M. Steroid withdrawal/avoidance: The Maastricht experience. . International Updates Transplantation, Permanyer. 2019.

5. Advagraf: scientific discussion. Available at: http://www.ema.europa.eu/Once-Daily Tacrolimus 1575. 2007.

6. Collin C, Boussaud V, Lefeuvre S, et al. Sublingual tacrolimus as an alternative to intravenous route in patients with thoracic transplant: a retrospective study. Transplant Proc 2010;42(10):4331-4337.

7. Kahan,BD. Cyclosporine. N Engl J Med 1989;321(25):1725-1738.

8. Vincenti F, Laskow DA, Neylan JF, et al. One-year follow-up of an open-label trial of FK506 for primary kidney transplantation. A report of the U.S. Multicenter FK506 Kidney Transplant Group. Transplantation 1996;61(11):1576-1581.

9. Coemans $M$, Susal C, Dohler B, et al. Analyses of the short- and long-term graft survival after kidney transplantation in Europe between 1986 and 2015. Kidney Int 2018;94(5):964-973.

10. Lamb KE, Lodhi S, Meier-Kriesche HU. Long-term renal allograft survival in the United States: a critical reappraisal. Am J Transplant 2011;11(3):450-462.

11. Meier-Kriesche HU, Schold JD, Srinivas TR. Lack of improvement in renal allograft survival despite a marked decrease in acute rejection rates over the most recent era. Am J Transplant 2004;4(3): 378-383.

12. Staatz CE, Tett SE. Clinical pharmacokinetics and pharmacodynamics of tacrolimus in solid organ transplantation. Clin Pharmacokinet 2004;43(10):623-653.

13. Beland MA, Lapointe I, Noel R, et al. Higher calcineurin inhibitor levels predict better kidney graft survival in patients with de novo donor-specific anti-HLA antibodies: a cohort study. Transpl Int 2017; 30(5):502-509.

14. Opelz G. Tacrolimus trough levels and graft survival. Collaborative Transplant Study Newsletter. 2014.

15. Susal C, Dohler B. Late intra-patient tacrolimus trough level variability as a major problem in kidney transplantation: A Collaborative Transplant Study Report. Am J Transplant 2019;19(10):2805-2813.

16. Kuypers DR, Claes K, Evenepoel P, et al. Time-related clinical determinants of long-term tacrolimus pharmacokinetics in combination therapy with mycophenolic acid and corticosteroids: a prospective study in one hundred de novo renal transplant recipients. Clin Pharmacokinet 2004;43(11):741-762.

17. Borra LC, Roodnat JI, Kal JA, et al. High within-patient variability in the clearance of tacrolimus is a risk factor for poor long-term outcome after kidney transplantation. Nephrol Dial Transplant 2010;25(8): 2757-2763.

18. Kahan BD, Welsh M, Schoenberg $L$, et al. Variable oral absorption of cyclosporine. A biopharmaceutical risk factor for chronic renal allograft rejection. Transplantation 1996;62(5): 599-606.

19. O'Regan JA, Canney M, Connaughton DM, et al. Tacrolimus trough-level variability predicts long-term allograft survival following kidney transplantation. J Nephrol 2016;29(2):269-276.

20. Rodrigo E, Segundo DS, Fernandez-Fresnedo G, et al. Within-Patient Variability in Tacrolimus Blood Levels Predicts Kidney Graft Loss and Donor-Specific Antibody Development. Transplantation 2016; 100(11):2479-2485.

21. Sapir-Pichhadze R, Wang Y, Famure O, et al. Time-dependent variability in tacrolimus trough blood levels is a risk factor for late kidney transplant failure. Kidney Int 2014;85(6):1404-1411.

22. Shuker N, Shuker L, van Rosmalen J, et al. A high intrapatient variability in tacrolimus exposure is associated with poor long-term outcome of kidney transplantation. Transpl Int 2016;29(11): 1158-1167. 
23. Whalen HR, Glen JA, Harkins V, et al. High Intrapatient Tacrolimus Variability Is Associated With Worse Outcomes in Renal Transplantation Using a Low-Dose Tacrolimus Immunosuppressive Regime. Transplantation 2017;101(2):430-436.

24. Boots JM, Christiaans $\mathrm{MH}$, Van Duijnhoven EM, et al. Early steroid withdrawal in renal transplantation with tacrolimus dual therapy: a pilot study. Transplantation 2002;74(12):1703-1709.

25. van Duijnhoven EM, Boots JM, Christiaans $\mathrm{MH}$, et al. Increase in tacrolimus trough levels after steroid withdrawal. Transpl Int 2003;16(10):721-725.

26. Reams D, Rea J, Davis D, et al. Utility of sublingual tacrolimus in cystic fibrosis patients after lung transplantation. J Heart Lung Transplant 2001;20(2):207-208.

27. van de Plas A, Dackus J, Christiaans $\mathrm{MH}$, et al. Pharmacokinetics of sublingual and rectal administration of tacrolimus: a pilot study. Pharmaceutisch Weekblad 2008;2(3):66-68.

28. Anglicheau D, Flamant $\mathrm{M}$, Schlageter $\mathrm{MH}$, et al. Pharmacokinetic interaction between corticosteroids and tacrolimus after renal transplantation. Nephrol Dial Transplant 2003;18(11):2409-2414.

29. Hesselink DA, Ngyuen $\mathrm{H}$, Wabbijn $\mathrm{M}$, et al. Tacrolimus dose requirement in renal transplant recipients is significantly higher when used in combination with corticosteroids. Br J Clin Pharmacol 2003;56(3): 327-330.

30. Zhang J, Kuehl P, Green ED, et al. The human pregnane $X$ receptor: genomic structure and identification and functional characterization of natural allelic variants. Pharmacogenetics 2001;11(7): 555-572.

31. Kliewer SA, Moore JT, Wade L, et al. An orphan nuclear receptor activated by pregnanes defines a novel steroid signaling pathway. Cell 1998;92(1):73-82.

32. Ma X, Idle JR, Gonzalez FJ. The pregnane X receptor: from bench to bedside. Expert Opin Drug Metab Toxicol 2008;4(7):895-908.

33. Burk O, Koch I, Raucy J, et al. The induction of cytochrome P450 3A5 (CYP3A5) in the human liver and intestine is mediated by the xenobiotic sensors pregnane $X$ receptor (PXR) and constitutively activated receptor (CAR). J Biol Chem 2004;279(37):38379-38385.

34. Benkali K, Premaud A, Picard N, et al. Tacrolimus population pharmacokinetic-pharmacogenetic analysis and Bayesian estimation in renal transplant recipients. Clin Pharmacokinet 2009;48(12): 805-816.

35. Hesselink DA, van Schaik RH, van Agteren $M$, et al. CYP3A5 genotype is not associated with a higher risk of acute rejection in tacrolimus-treated renal transplant recipients. Pharmacogenet Genomics 2008;18(4):339-348.

36. Macphee IA, Fredericks S, Mohamed M, et al. Tacrolimus pharmacogenetics: the CYP3A5*1 allele predicts low dose-normalized tacrolimus blood concentrations in whites and South Asians. Transplantation 2005;79(4):499-502.

37. Haller MC, Royuela A, Nagler EV, et al. Steroid avoidance or withdrawal for kidney transplant recipients. Cochrane Database Syst Rev 2016;(8):CD005632.

38. Pascual J, van Hooff JP, Salmela K, et al. Three-year observational follow-up of a multicenter, randomized trial on tacrolimus-based therapy with withdrawal of steroids or mycophenolate mofetil after renal transplant. Transplantation 2006;82(1):55-61.

39. Maes BD, Claes K, Coosemans W, et al. Cessation of steroids in stable renal transplant patients: the Leuven experience. Clin Transpl: 2002:181-189.

40. Vanrenterghem Y, van Hooff JP, Squifflet JP, et al. Minimization of immunosuppressive therapy after renal transplantation: results of a randomized controlled trial. Am J Transplant 2005;5(1):87-95.

41. Nicolai S, Bunyavanich S. Hypersensitivity reaction to intravenous but not oral tacrolimus. Transplantation 2012;94(9):e61-63.

42. van Dieren JM, van Bodegraven AA, Kuipers EJ, et al. Local application of tacrolimus in distal colitis: feasible and safe. Inflamm Bowel Dis 2009;15(2):193-198.

43. van de Plas A, Dackus J, Christiaans $\mathrm{MH}$, et al. A pilot study on sublingual administration of tacrolimus. Transpl Int 2009;22(3): 358-359.

44. Nasiri-Toosi Z, Dashti-Khavidaki S, Nasiri-Toosi M, et al. Clinical pharmacokinetics of oral versus sublingual administration of tacrolimus in adult liver transplant recipients. Exp Clin Transplant 2012; 10(6):586-591. 
45. Romero I, Jimenez C, Gil F, et al. Sublingual administration of tacrolimus in a renal transplant patient. J Clin Pharm Ther 2008;33(1):87-89.

46. Undre NA, Stevenson P, Schafer A. Pharmacokinetics of tacrolimus: clinically relevant aspects. Transplant Proc 1999;31(7A):21S-24S.

47. Venkataramanan R, Jain A, Warty VS, et al. Pharmacokinetics of FK 506 in transplant patients. Transplant Proc 1991;23(6):2736-2740.

48. Zhang $\mathrm{H}$, Zhang J, Streisand JB. Oral mucosal drug delivery: clinical pharmacokinetics and therapeutic applications. Clin Pharmacokinet 2002;41(9):661-680.

49. Federico S, Carrano R, Sabbatini M, et al. Sublingual administration improves systemic exposure of tacrolimus in kidney transplant recipients: comparison with oral administration. Eur J Clin Invest 2016;46(7):651-657.

50. Note for Guidance on the Investigation of Bioavailability and Bioequivalence (NfG on BA BE). E. M. Agency. London. 2010.

51. Jaeger SU, Klag T, Hoeger K, et al. Tacrolimus Suppositories in Therapy-Resistant Ulcerative Proctitis. Inflamm Intest Dis 2019;3(3):116-124.

52. Zhang $\mathrm{Y}$, Benet LZ. The gut as a barrier to drug absorption: combined role of cytochrome P450 $3 \mathrm{~A}$ and P-glycoprotein. Clin Pharmacokinet 2001;40(3):159-168.

53. Fojo AT, Ueda K, Slamon DJ, et al. Expression of a multidrug-resistance gene in human tumors and tissues. Proc Natl Acad Sci U S A 1987;84(1):265-269.

54. Thorn M, Finnstrom N, Lundgren S, et al. Cytochromes P450 and MDR1 mRNA expression along the human gastrointestinal tract. Br J Clin Pharmacol 2005;60(1):54-60.

55. Paine MF, Hart HL, Ludington SS, et al. The human intestinal cytochrome P450 "pie". Drug Metab Dispos 2006;34(5):880-886.

56. Wacher VJ, Silverman JA, Zhang Y, et al. Role of P-glycoprotein and cytochrome P450 3A in limiting oral absorption of peptides and peptidomimetics. J Pharm Sci 1998;87(11):1322-1330.

57. Wallemacq $\mathrm{P}$, Armstrong VW, Brunet $\mathrm{M}$, et al. Opportunities to optimize tacrolimus therapy in solid organ transplantation: report of the European consensus conference. Ther Drug Monit 2009;31(2): 139-152.

58. Alloway R, Steinberg S, Khalil K, et al. Conversion of stable kidney transplant recipients from a twice daily Prograf-based regimen to a once daily modified release tacrolimus-based regimen. Transplant Proc 2005;37(2):867-870.

59. Pisitkun T, Eiam-Ong S, Chusil S, et al. The roles of $\mathrm{C} 4$ and AUCO-4 in monitoring of tacrolimus in stable kidney transplant patients. Transplant Proc 2002;34(8):3173-3175.

60. Stolk LM, Van Duijnhoven EM, Christiaans $\mathrm{MH}$, et al. Trough levels of tacrolimus. Ther Drug Monit 2002;24(4): 573; author reply 573-574.

61. Wong KM, Shek CC, Chau KF, et al. Abbreviated tacrolimus area-under-the-curve monitoring for renal transplant recipients. Am J Kidney Dis 2000;35(4):660-666.

62. Op den Buijsch RA, van de Plas A, Stolk LM, et al. Evaluation of limited sampling strategies for tacrolimus. Eur J Clin Pharmacol 2007;63(11):1039-1044.

63. Hoogtanders K, van der Heijden J, Christiaans M, et al. Therapeutic drug monitoring of tacrolimus with the dried blood spot method. J Pharm Biomed Anal 2007;44(3):658-664.

64. Bekersky I, Dressler D, Mekki QA. Effect of low- and high-fat meals on tacrolimus absorption following $5 \mathrm{mg}$ single oral doses to healthy human subjects. J Clin Pharmacol 2001;41(2):176-182.

65. Prograf: Summary of Product Characteristics (2006). Available at: https://http://www.ema.europa.eu/ en/documents/referral/prograf-article-30-referral-annex-i-ii-iii_en.pdf

66. van Duijnhoven EM, Christiaans $\mathrm{MH}$, Boots JM, et al. Evidence that fasting does not significantly affect trough levels of tacrolimus in stable renal transplant recipients. Transplant Proc 2002;34(5):1723-1725.

67. Christiaans $M$, van Duijnhoven E, Beysens $T$, et al. Effect of breakfast on the oral bioavailability of tacrolimus and changes in pharmacokinetics at different times posttransplant in renal transplant recipients. Transplant Proc 1998;30(4):1271-1273.

68. van Duijnhoven E, Christiaans M, Undre N, et al. The effect of breakfast on the oral bioavailability of tacrolimus in diabetic and nondiabetic patients before transplantation. Transplant Proc 1998;30(4): 1268-1270. 
69. Advagraf: Summary of Product Characteristics (2009). Available at: https://http://www.ema.europa.eu/ en/documents/product-information/advagraf-epar-product-information_en.pdf

70. van Gelder T, van Schaik RH, Hesselink DA. Pharmacogenetics and immunosuppressive drugs in solid organ transplantation. Nat Rev Nephrol 2014;10(12):725-731.

71. Kurnatowska I, Krawczyk J, Oleksik T, et al. Tacrolimus dose and blood concentration variability in kidney transplant recipients undergoing conversion from twice daily to once daily modified release tacrolimus. Transplant Proc 2011;43(8):2954-2956.

72. Shuker N, van Gelder T, Hesselink DA. Intra-patient variability in tacrolimus exposure: causes, consequences for clinical management. Transplant Rev (Orlando) 2015;29(2):78-84.

73. Wehland $M$, Bauer S, Brakemeier S, et al. Differential impact of the CYP3A5* 1 and CYP3A5*3 alleles on pre-dose concentrations of two tacrolimus formulations. Pharmacogenet Genomics 2011;21(4): 179-184.

74. Wu MJ, Cheng $\mathrm{CY}$, Chen $\mathrm{CH}$, et al. Lower variability of tacrolimus trough concentration after conversion from prograf to advagraf in stable kidney transplant recipients. Transplantation 2011; 92(6):648-652.

75. Shuker N, Cadogan M, van Gelder T, et al. Conversion from twice-daily to once-daily tacrolimus does not reduce intrapatient variability in tacrolimus exposure. Ther Drug Monit 2015;37(2):262-269.

76. Butler JA, Roderick $\mathrm{P}$, Mullee $\mathrm{M}$, et al. Frequency and impact of nonadherence to immunosuppressants after renal transplantation: a systematic review. Transplantation 2004;77(5): 769-776.

77. Wiebe C, Gibson IW, Blydt-Hansen TD, et al. Evolution and clinical pathologic correlations of de novo donor-specific HLA antibody post kidney transplant. Am J Transplant 2012;12(5):1157-1167.

78. Vanhove $T$, Vermeulen $T$, Annaert $P$, et al. High Intrapatient Variability of Tacrolimus Concentrations Predicts Accelerated Progression of Chronic Histologic Lesions in Renal Recipients. Am J Transplant 2016;16(10):2954-2963.

79. Wu MJ, Chang $\mathrm{CH}$, Cheng $\mathrm{CY}$, et al. Reduced variability of tacrolimus trough level in once-daily tacrolimus-based Taiwanese kidney transplant recipients with high-expressive genotype of cytochrome P450 3A5. Transplant Proc 2014;46(2):403-405.

80. Cheung CY, Chan KM, Wong YT, et al. Impact of CYP3A5 Genetic Polymorphism on Intrapatient Variability of Tacrolimus Exposure in Chinese Kidney Transplant Recipients. Transplant Proc 2019; 51(6):1754-1757.

81. Kuypers DR, Peeters PC, Sennesael JJ, et al. Improved adherence to tacrolimus once-daily formulation in renal recipients: a randomized controlled trial using electronic monitoring. Transplantation 2013; 95(2):333-340.

82. Knight SR. Intrapatient variability in tacrolimus exposure - a useful tool for clinical practice? Transpl Int 2016;29(11):1155-1157.

83. Kuypers DRJ. Intrapatient Variability of Tacrolimus Exposure in Solid Organ Transplantation: A Novel Marker for Clinical Outcome. Clin Pharmacol Ther 2019;107(2):347-358.

84. Srinarong $\mathrm{P}$, Pham BT, Holen $\mathrm{M}$, et al. Preparation and physicochemical evaluation of a new tacrolimus tablet formulation for sublingual administration. Drug Dev Ind Pharm. 2011.

85. Pallet N, Etienne I, Buchler M, et al. Long-Term Clinical Impact of Adaptation of Initial Tacrolimus Dosing to CYP3A5 Genotype. Am J Transplant 2016;16(9):2670-2675.

86. van der Wouden $\mathrm{CH}$, van Rhenen $\mathrm{MH}$, Jama WOM, et al. Development of the PGx-Passport: A Panel of Actionable Germline Genetic Variants for Pre-Emptive Pharmacogenetic Testing. Clin Pharmacol Ther 2019;106(4):866-873.

87. Benkali K, Rostaing L, Premaud A, et al. Population pharmacokinetics and Bayesian estimation of tacrolimus exposure in renal transplant recipients on a new once-daily formulation. Clin Pharmacokinet 2010;49(10):683-692.

88. Goodall DL, Willicombe M, McLean AG, et al. High Intrapatient Variability of Tacrolimus Levels and Outpatient Clinic Nonattendance Are Associated With Inferior Outcomes in Renal Transplant Patients. Transplant Direct 2017;3(8):e192.

89. Hoogtanders K, van der Heijden J, Christiaans M, et al. Dried blood spot measurement of tacrolimus is promising for patient monitoring. Transplantation 2007;83(2):237-238. 

Nederlandse samenvatting 



\section{Introductie}

Niertransplantatie is de beste nierfunctie vervangende therapie voor patiënten met eindstadium nierfalen. In Nederland werden in 2018998 niertransplantaties verricht. In totaal waren 11405 patiënten in leven met een functionerend niertransplantaat. Ter voorkoming van een afstotingsreactie zijn immuunsuppressiva noodzakelijk.

Tacrolimus is tegenwoordig daarbij het hoofdbestanddeel van de immuunsuppressieve therapie. Het middel wordt afhankelijk van het vingerende protocol gecombineerd met andere middelen zoals mycofenolaat/mycofenolzuur, mTORremmers (sirolimus of everolimus), maar ook met steroïden (prednisolon). Tacrolimus bestaat als een tweemaal daagse formulering (Prograf(t): Tac BID en een eenmaal daagse formulering met gereguleerde afgifte (Advagraf: Tac QD). Er is ook een intraveneuze toedieningsvorm beschikbaar voor het geval dat orale inname niet mogelijk is. Echter, intraveneuze toediening kan problematisch zijn i.v.m foutieve dosisberekeningen en anafylactische reacties op bestanddelen uit de oplossing en/of de infuuslijnen.

Tacrolimus heeft een smalle therapeutische breedte, d.w.z. er is een nauwe marge tussen een te lage spiegel met gevaar voor afstoting, en een te hoge spiegel, gepaard gaande met meer toxiciteit van het medicijn. Daarnaast bestaan er grote individuele verschillen in de mate van absorptie en metabolisme van het middel, hetgeen o.a. wordt beïnvloed door activiteit van het cytochroom P450 3A5 (Cyp3A5). De tacrolimus streefwaarde en de farmacokinetiek veranderen tevens in het beloop na transplantatie. Een grote variabiliteit in tacrolimus spiegels is inmiddels een bewezen risicofactor voor verlies van het transplantaat op de lange termijn. Strikte spiegel controle als een maat voor blootstelling d.m.v. 'therapeutic drug monitoring' (TDM) is hierbij belangrijk.

In dit proefschrift werden verschillende farmacokinetische en farmaco-genetische aspecten van tacrolimus therapie onderzocht. Enerzijds werden farmacokinetische aspecten van de twee maal daagse formulering Tac BID bestudeerd (Deel I: Hoofdstukken 2 en 3). Anderzijds werd de farmacokinetiek van de nieuwere Tac QDformulering vanuit een klinisch relevant perspectief bekeken (Deel II: Hoofdstukken $4 \mathrm{t} / \mathrm{m}$ 6).

\section{Deel I}

Het post-transplantatie beleid binnen het Maastrichtse transplantatiecentrum wordt gekenmerkt door het vroeg afbouwen van steroïden (prednisolon) na een ongecompliceerde transplantatie. Opvallenderwijs, neemt de tacrolimus behoefte na het afbouwen van steroïden af en stijgt de voor de dosis gecorrigeerde tacrolimus dalspiegel $\left(\mathrm{DnC}_{0}\right)$. Hierbij zijn er echter grote interindividuele verschillen waarneembaar, waarvan men niet weet waarom deze verschillen bestaan. 
Steroïden induceren de Сур3A enzymen. Er werd daarom verondersteld dat het afbouwen van steroïden tot verminderde activiteit van deze enzymen leidt, waardoor er dus een lagere tacrolimus dosis nodig is. Een andere mogelijke factor die hierbij betrokken zou kunnen zijn, betreft de Pregnane X Receptor (PXR). Het is een eiwit met een steroïdreceptor dat veel geneesmiddel metaboliserende enzymsystemen (zoals het Сур3А systeem) en transporteiwitten reguleert. Daarom zouden genetische polymorfismen (SNP'S ) van het PXR-gen ook van invloed kunnen zijn op het tacrolimus metabolisme bij het afbouwen van steroïden. In Hoofdstuk 2 werd onderzocht in hoeverre SNP's van het PXR-gen en het Cyp3A5 enzym hierop van invloed zijn.

In een cohort van 83 niertransplantatiepatiënten werden de veranderingen in de $\mathrm{DnC}_{0}$ bij het afbouwen van de prednisolon en na het stoppen ervan vergeleken tussen verschillende genotypen van het Cyp3A5 en het PXR gen. Patiënten met het Cyp3A5*1 genotype (zogenaamde fast metabolizers) hadden een lagere $\mathrm{DnC}_{0}$ in vergelijking met Cyp 3A5*3-homozygote patiënten (zogenaamde slow metabolizers), zowel tijdens als na steroïd therapie. Cyp3A5 *1 dragers toonden tevens een significant minder sterke stijging van de $\mathrm{DnC}_{0}$ na het afbouwen van steroïden vergeleken met Cyp3A5 *3 homozygoten (+33 vs. +66\%). Ook het PXR-gen speelt een rol in de farmacokinetiek van tacrolimus: de mate van verandering van de $\mathrm{DnC}_{0}$ na het afbouwen van steroïden is afhankelijk van het PXR 7635A>G SNP. De correlaties tussen de SNP's van het PXR 7635A>G en het Cyp3A5-gen en de mate van verandering in de $\mathrm{DnC}_{0}$ zijn onafhankelijk van elkaar.

Deze bevindingen verklaren (een deel van) de interindividuele verschillen in de tacrolimus behoefte na het afbouwen van de steroïden. Anderzijds, dient de clinicus ook rekening te houden met een veranderde farmacokinetiek zodra steroïden worden voorgeschreven voor andere indicaties. Dit benadrukt tevens de noodzaak voor TDM na dosisaanpassingen van steroïden.

In Hoofdstuk 3, werden sublinguale en rectale toediening van tacrolimus onderzocht. Dit zou belangrijk kunnen zijn in geval dat normale orale inname niet mogelijk is. In een eerdere studie werden therapeutische spiegels beschreven na sublinguale toediening, hetgeen echter niet bevestigd kon worden in een pilotstudie die door onze groep werd verricht. Anderzijds, werden in deze pilotstudie wel significante tacrolimus spiegels gemeten na rectale toediening via een zetpil. De relatieve biologische beschikbaarheid van tacrolimus na sublinguale en rectale toediening werd vergeleken met eenmalige reguliere toediening van de Tac BID-formulering (Hoofdstuk 3). Bij dit onderzoek werden de drie verschillende tacrolimus formuleringen bij 18 gezonde vrijwilligers in verschillende volgorde toegepast.

$\mathrm{Na}$ sublinguale toediening konden in tegenstelling tot andere klinische studies geen therapeutische tacrolimus spiegels worden gemeten. Wij veronderstellen dat de therapeutische spiegels in de andere studies waarschijnlijk niet door daadwerkelijke 
sublinguale maar door reguliere gastro-intestinale absorptie werden bereikt. Onze proefpersonen waren onder strikte controle: zij mochten gedurende 15 minuten na toediening niet slikken met maatregelen tegen een late absorptie. ledere slikactie werd geregistreerd. Soortgelijke maatregelen en controles waren niet gegeven in de klinische studies. De tacrolimus curves in de eerder beschreven studies vertoonden tevens een profiel dat meer overeenkomt met gastro-intestinale dan met sublinguale absorptie. Omdat wij normale gastro-intestinale absorptie in de eerder beschreven studies veronderstellen, betekent dit ook dat men onder klinische omstandigheden zou moeten overwegen om bij tacrolimus eerder voor een enterale (b.v. via een sonde) dan voor een vaak bezwaarlijke parenterale (i.v.) toedieningsvorm te kiezen.

De rectale toediening zoals onderzocht in Hoofdstuk 3 daarentegen gaf wél significante spiegels. Rectale toediening van tacrolimus zou dus een alternatief voor de orale route kunnen zijn, waarbij hogere doseringen dienen te worden toegepast gezien de lagere relatieve biologische beschikbaarheid in onze studie. Naast te verwachten systemische effecten zullen echter ook de bijwerkingen kunnen optreden. lets waarvan men zich bewust dient te zijn wanneer tacrolimus wordt toegepast voor andere doeleinden zoals locale behandeling van een refractaire colitis. Ook dient rekening te worden gehouden met een grotere variabiliteit na rectale toediening. Toekomstige farmacokinetische studies dienen ook te worden uitgevoerd bij transplantatiepatiënten. Zolang deze studies er niet zijn, dient rectale toediening van tacrolimus onder supervisie van expertise te gebeuren met regelmatige TDM.

\section{Deel II}

In Hoofdstuk 4 werden strategieën onderzocht hoe de $\mathrm{AUC}_{0-24}$ van TAC QD het beste voorspeld zou kunnen worden. Zoals eerder vermeld, heeft tacrolimus een smalle therapeutische breedte en is TDM aangewezen. Voor Tac BID is de $\mathrm{AUC}_{0-12}$ de gouden standaard. Omdat dit in de praktijk moeilijk te realiseren is, wordt de dalspiegel $\left(C_{0}\right)$ als surrogaat gebruikt gezien de goede correlatie die er zou bestaan tussen deze waarde en de AUC. Er wordt echter getwijfeld aan de voorspellende waarde van de $\mathrm{C}_{0}$ voor de AUC van Tac BID. Daarom zijn er 'Limited Sampling Strategieën (LSS's) met twee of drie meetmomenten (meestal binnen enkele uren na inname van het medicijn) ontwikkeld die een betere voorspelling van de Tac BID-AUC zouden geven. Terwijl voor Tac QD ook een goede correlatie tussen de $C_{0}$ en de $A U_{0-24}$ wordt gerapporteerd, is het echter nog onbekend of andere monitoring strategieën een betere voorspelling kunnen geven voor de $\mathrm{AUC}_{0-24}$ bij dit middel met een vertraagde afgifte. Het is tevens aannemelijk dat de tekortkomingen van de $C_{0}$ voor voorspelling van de AUC van Tac BID ook van toepassing zullen zijn bij Tac QD. In Hoofdstuk 4 werden LSS's voor de voorspelling van de $A U_{0-24}$ van Tac QD ontwikkeld en in een vroege ( 2 weken) en een late ( $>6$ maanden) fase na niertransplantatie gevalideerd. De LSS's zijn gebaseerd op lineaire regressieanalyse (LRA). De voorspellende waarde werd 
tevens vergeleken met een populatiekinetisch model (Maximum A Posteriori Bayesian estimation; MAPB).

Uit de LRA blijkt dat bij de late validatiegroep (>6 maanden na transplantatie) een enkele spiegel gemeten in de eliminatiefase 8 uur na inname van Tac QD $\left(C_{8}\right)$ een accurate voorspelling van de $A \mathrm{CC}_{0-24}$ geeft. MAPB bevestigde de noodzaak voor laattijdige meting. Geen van de door de LRA vastgestelde LSS's bevatte een dalspiegel $\left(C_{0}\right.$ dan wel $\left.C_{24}\right)$, tenzij deze metingen geforceerd in het predictiemodel werden opgenomen, in combinatie met andere tijdstippen.

$\mathrm{Bij}$ de vroege validatiegroep volstond een strategie met een eenmalige meting niet om de $\mathrm{AUC}_{0-24}$ adequaat te voorspellen. Hierbij gaf een meting op drie tijdstippen de beste voorspelling. Naast twee metingen in de eliminatiefase $\left(C_{8}\right.$ en $\left.C_{12}\right)$ werd een meting rondom de piekconcentratie $\left(C_{2}\right)$ aan het model toegevoegd. In de vroege fase na transplantatie, wanneer de kansen van een afstotingsreactie het hoogste zijn, is voorspelling van de $\mathrm{AUC}_{0-24}$ dus niet zonder meer gegeven door meting van de dalspiegel. Hiervoor dient vaker en ook later gemeten te worden. Dit is haalbaar zolang de patiënt klinisch is, maar voor poliklinische controle is dit onpraktisch. Deze beperking zou d.m.v. een capillaire bloedafname zoals de dried blood spot (DBS) methode opgelost kunnen worden.

In Hoofdstuk 5 werd bij stabiele niertransplantatiepatiënten naar de invloed van een continentaal ontbijt op de farmacokinetiek van Tac QD gekeken. $\mathrm{Er}$ is een verminderde absorptie van Tac BID na inname met voedsel beschreven, m.n na een vetrijke maaltijd. Daarom wordt nuchtere inname van het medicijn (d.w.z. één uur vóór of twee uur na een maaltijd) aanbevolen. Dit is niet erg patiëntvriendelijk en zou kunnen bijdragen tot verminderde therapietrouw.

In eerdere studies was reeds beschreven dat de $\mathrm{AUC}_{0-24}$ van Tac BID bij de meeste patiënten getalsmatig maar niet klinisch relevant verlaagd was wanneer het medicijn samen met een ontbijt werd ingenomen. Het effect van voedsel was drie weken na de transplantatie het geringst. Omwille van de patiëntvriendelijkheid wordt patiënten in het Maastrichtse centrum daarom vanaf de derde week na de transplantatie toegestaan de Tac BID samen met het ontbijt in te nemen, mits er stabiele spiegels zijn.

Ook voor Tac QD wordt nuchtere inname aanbevolen. Volgens de product bijsluiter is er anders een verminderde absorptie van Tac $Q D$, hetgeen is onderzocht bij gezonde vrijwilligers. Bij transplantatiepatiënten waren nog geen farmacokinetische data over de invloed van voedselinname beschikbaar. In Hoofdstuk 5 worden de resultaten beschreven van een prospectieve farmacokinetische studie naar de invloed van een ontbijt op de relatieve biologische beschikbaarheid van Tac QD ten opzichte van nuchtere inname.

Na niet nuchtere inname van Tac QD werd een $14 \%$ lagere $\mathrm{AUC}_{0-24}$ gemeten met een verlenging van de $\mathrm{T}_{\max }$ met 1 uur. De mate van verandering in de $\mathrm{AUC}_{0-24}$ na niet 
nuchtere inname toonde een brede spreiding van $-38 \%$ tot $+29 \%$. In de meerderheid van de gevallen (59\%) was de verandering in $\mathrm{AUC}_{0-24}$ kleiner dan $20 \%$, hetgeen arbitrair als grenswaarde van relevante verandering werd beschouwd. Bij patiënten met het Cyp3A5*1 genotype leek het effect van voedsel op de $\mathrm{AUC}_{0-24}$ minder uitgesproken, maar omdat het aantal Cyp3A5*1-dragers $(n=5)$ zeer beperkt was, kan hier geen definitieve uitspraak over worden gedaan. Deze bevinding dient in een grotere Cyp3A5*1-populatie bevestigd te worden.

Op basis van onze bevindingen wordt vanaf ongeveer 3 weken na transplantatie ook Tac QD-inname samen met een ontbijt met een relatief laag vetgehalte toegestaan onder de voorwaarde van een stabiele nierfunctie en stabiele spiegels. Gezien de grote spreiding in de potentiële verandering na conversie dient een tacrolimus spiegel 1 week nadien (wanneer een steady state is bereikt) te worden gecontroleerd. In hoeverre niet nuchtere inname van tacrolimus daadwerkelijk de therapietrouw bevordert, dient nog bewezen te worden.

Tot slot werd in Hoofdstuk 6 de verandering in intra-patiënt variabiliteit (IPV) na conversie van Tac BID naar Tac QD onderzocht. Deze studie was van groot belang omdat een hoge IPV gepaard gaat met een verminderde overleving van het niertransplantaat. Eén van de factoren die van invloed kan zijn op de IPV betreft verschil in formuleringen. Beperkingen van de eerder gepubliceerde studies hierover waren het retrospectieve design, het gebruik van de dalspiegel als surrogaat voor blootstelling of het beperkt aantal metingen van de $\mathrm{AUC}_{0-24}$.

Daarom werd een prospectieve farmacokinetische studie in 40 stabiele niertransplantatiepatiënten verricht. Hierbij werd de IPV van de $\mathrm{AUC}_{0-24}$ onderzocht na conversie naar Tac QD. Voor beide formuleringen werden bij farmacokinetisch evenwicht vijf 24-uurs curves verzameld d.m.v. de DBS-methode, elk bestaande uit 8 meetmomenten. Daarnaast werd in deze studie de invloed van het Cyp3A5 systeem bestudeerd.

Na conversie van Tac BID naar Tac QD daalde de IPV van 14,1\% naar 10,9\% (22\% relatieve daling). Opvallenderwijs was de IPV reeds laag onder Tac BID bij deze a priori zeer therapietrouwe patiëntpopulatie, blijkende uit de 24-uurs curves. Therapieontrouw is derhalve nagenoeg uitgesloten in deze studie zodat de daling van de IPV een intrinsiek farmacokinetisch verschil tussen de twee formuleringen betekent. Bij Cyp3A5*1-dragers was de daling in IPV nog sterker: van 18.2\% naar $12.8 \%$ (30\% relatieve daling). In hoeverre het Cyp3A5 genotype hierbij daadwerkelijk een rol speelt, is op basis van de huidige literatuur tegenstrijdig. Toekomstige prospectieve studies zouden moeten uitwijzen of Cyp3A5*1-dragers daadwerkelijk het meest gebaat zouden zijn bij conversie naar Tac QD.

Concluderend, is dit tot dusver de enige prospectieve studie die o.b.v. herhaaldelijke 24-uurs metingen robuuste data heeft verzameld aangaande de $\mathrm{AUC}_{0-24}$-IPV van Tac BID en Tac QD. Naast een verbeterde therapietrouw draagt ook de farmacokinetiek 
van Tac QD bij aan de lagere IPV. Gezien het belang van de IPV voor de lange-termijn transplantaatoverleving zou bij controle niet slechts de dalspiegel maar ook de IPV moeten worden gemeten. Een hoge IPV zou een risicofactor voor de patiënt betekenen en in dat geval zou naar een oorzaak gezocht moeten worden.

\section{Conclusies, toekomstperspectieven en onzekerheden}

Dit proefschift geeft nieuwe inzichten in verschillende facetten van tacrolimus therapie. Bestudeerd werden de invloed van genetische polymorfismen van het PXRen het Cyp3A5-gen op het afbouwen van steroïden, alternatieve toedieningsroutes, TDM-strategieën voor Tac QD, de invloed van voedsel en de verschillen in IPV tussen Tac BID en Tac QD. De resultaten van dit proefschrift zijn gebaseerd op patiënten vanuit het Maastrichtse transplantatiecentrum dat gekenmerkt wordt door een hoge prevalentie van tacrolimus monotherapie ten opzichte van andere transplantatiecentra. In hoeverre dit gegeven van invloed is op de uitkomsten, is onduidelijk.

Vanuit farmacogenetisch oogpunt werd een invloed van PXR- en Cyp3A5-SNP's op de mate van stijging van de $\mathrm{DnC}_{0}$ aangetoond. Bepaling van het genotype vóór transplantatie wordt nog niet aanbevolen, maar onderzoeken naar een farmacogenetisch paspoort zijn gaande. Mogelijke genetische invloeden op de interindividuele verschillen bij het afbouwen of het voorschijven van steroïden dienen overwogen te worden. Daarom is hierbij TDM strikt noodzakelijk.

Het werd aangetoond dat er geen echte sublinguale absorptie van tacrolimus is. Hoewel herhaaldelijke of hogere doseringen tot meetbare tacrolimus spiegels zouden kunnen leiden, lijkt dit op basis van onze bevindingen en ervaringen onwaarschijnlijk. Toevoegen van een hydrofiele drager zou orale mucosale absorptie door verbeterde wateroplosbaarheid mogelijk kunnen maken, maar dient verder onderzocht te worden.

Rectale toediening lijkt op basis van de studie bij gezonde proefpersonen haalbaar te zijn. Toekomstige studies dienen verdere inzichten te verschaffen in de farmacokinetiek bij transplantatiepatiënten. Bij colitis patiënten, waar dit middel offlabel wordt toegepast, dient men rekening te houden met mogelijke bijwerkingen. TDM is onder alle omstandigheden aangewezen.

Op het gebied van TDM werd aangetoond dat bij niertransplantatiepatiënten in de onderhoudsfase, meting van de 8 -uurs spiegel $\left(C_{8}\right)$ een adequatere voorspelling geeft van de Tac QD-AUC $C_{0-24}$ dan een dalspiegel. Vroeg na transplantatie was de $A_{U C} C_{0-24}$ slecht voorspelbaar d.m.v een dalspiegel, in tegenstelling tot de 3-punts meting van $\mathrm{C}_{2}, \mathrm{C}_{8}$ en $\mathrm{C}_{12}$. Bezwaren tegen vaker of later meten kunnen worden overwonnen door gebruik te maken van de dried blood spot (DBS). Tot dusver, zijn er echter geen studies die hebben aangetoond dat TDM o.b.v. AUC-metingen een betere patiënt- of transplantaatuitkomst geeft. 
Van de inname van Tac QD samen met een ontbijt dat relatief weinig vet bevat, werd aangetoond dat dit gemiddeld tot een milde afname van de $\mathrm{AUC}_{0-24}$ en de dalspiegel leidt. Daarom wordt patiënten toegestaan Tac QD ook niet nuchter in te nemen onder spiegelcontrole, gegeven de grote interindividuele spreiding. In hoeverre deze maatregel met een betere therapietrouw gepaard gaat moet nog aangetoond worden. Ongeacht of de patiënt Tac QD nuchter of niet nuchter inneemt, dient het consistent op dezelfde manier te gebeuren. Als voor niet nuchtere inname wordt gekozen, dient er een nauwe tijdsrelatie tussen inname van het voedsel en het medicijn te zijn. Frequente veranderingen in deze kunnen met een hogere variabiliteit gepaard gaan.

In een prospectieve farmacokinetische studie werd aangetoond dat ook in een strikt therapietrouwe populatie de IPV van de $A_{U C} C_{0-24}$ afneemt onder Tac QD. Dit is belangrijk gezien de negatieve invloed van een hoge IPV op transplantaat overleving. Het is aannemelijk dat therapieontrouw de belangrijkste oorzaak is voor een hoge IPV. Het is onbekend en of een lagere intrinsieke farmacokinetische IPV naast verbeterde therapietrouw bijdraagt aan een betere lange-termijn transplantaatoverleving.

De studie in Hoofdstuk 6 heeft ook aangetoond dat de DBS-methode een belangrijk gereedschap kan zijn voor het uitvoeren van wetenschappelijk onderzoek. Door de DBS zijn frequentere en latere metingen mogelijk en levert hierdoor een meetinstrument voor de IPV. Patiënten met een hoge IPV kunnen worden geïdentificeerd en interventies kunnen worden toegepast. De DBS-methode kan door de patiënt zelf thuis worden uitgevoerd en draagt op deze manier bij aan het concept van geïndividualiseerde patiëntenzorg. 

Valorisation 



\section{Valorisation}

\subsection{What is the relevance of improving tacrolimus therapy?}

Chronic kidney disease is a major burden for public health. Patients with end stage renal disease (ESRD) have to undergo renal replacement therapy. Dialysis is one of the modalities with annual costs of approximately $€ 80,000$ per patient in the Netherlands (https://nieratlas.nl). Renal transplantation comes with better patient survival, better quality of life and lower costs as from one year after transplantation. ${ }^{1,2}$ Annually, each year of survival with a functioning kidney graft translates into a saving of about $€ 60,000$ per patient compared with hemodialysis treatment (https://nieratlas.nl). For all these reasons, for patients with ESRD that are eligible for renal transplantation, this is the modality of choice, and prolonging graft survival is of utmost importance. The main reasons for transplant failure are 1 . death of the patient (especially due to cardiovascular, malignant and infectious diseases), 2. Rejection of the allograft. Both are related to the use of immunosuppressive drugs.

In 2018, more than 11,000 patients were alive with a functioning renal allograft in the Netherlands. Tacrolimus-based therapy is a cornerstone of the post-transplant care: about $90 \%$ of all transplant patients are on a tacrolimus-based immunosuppressive regimen. Optimizing tacrolimus therapy might contribute to a better graft survival and this can only be achieved by extending the knowledge of the drug, also from a clinical viewpoint which has been the main focus of this thesis.

\subsection{Which target groups are addressed in this thesis?}

The studies presented in this thesis have mainly been conducted in kidney transplant recipients. The conclusions drawn in this thesis with its practical recommendations aim at optimizing tacrolimus therapy as part of improved kidney transplant patient management. The patients themselves profit on the short-term through more convenient drug intake (i.e. once-daily dosing and consistent non-fasted intake) and home-based strategies for therapeutic drug monitoring (TDM). But also, patients suffering from refractory colitis that are sometimes treated with rectal tacrolimus may profit from the precautions recommended in this thesis.

Apart from the patients, the practical recommendations presented in this thesis are important for any clinician involved at some point in the care for the tacrolimustreated patient. This can be the nephrologist, the gastro-enterologist treating patients with colitis, the intensive care physician treating the sedated and intubated transplant patient, the general physician treating the transplant patient with corticosteroids for indications like e.g. chronic obstructive lung disease, gout or other inflammatory diseases. 


\subsection{Which activities may develop?}

This thesis delivers simple tools to identify the kidney transplant patient at increased risk for graft loss. During each regular outpatient control, the treating physician should routinely monitor the trough level together with the IPV, which can be determined from the coefficient of variation (CV). If both lie within a predefined target, no further action would be required except regular control. In case the trough level is outside the target range, tacrolimus dose should of course be adapted. At the same time, the physician should also determine the IPV. This requires repeated trough level measurements. These can be randomly ordered and done at home by the patients themselves by means of the Dried Blood Spot (DBS) method.

The clinician should be sensitized if tacrolimus levels depict a high IPV (Figure 9.1). This should prompt a search for the potential causes (Table 9.1). The search warrants a thorough anamnesis and a medication check, but also repeated randomly ordered home-based tacrolimus (trough) level measurement might provide important information. The anamnesis should focus on therapy adherence (i.e. intake of the correct dose at the correct time), changes in nutritional behaviors (randomly switching between fasted and non-fasted drug intake, randomly switching the time relation between food- and drug intake, changes in food fat content) or on intercurrent (diarrheal) illness. Contacting the pharmacy may be required to provide information considering correct delivery of the medication or use of other medication than registered in the patient file. Especially, there should be awareness of interacting co-medication. The required information could be provided by (online) questionnaires and/or by e-health applications and implemented in algorithms for risk stratification with subsequent awareness and implementation of interventions, when necessary.

Besides, on indication, determination of the patient's genotype may be of interest for future treatments that may help to better anticipate drug therapy. In this thesis, this mainly concerns the Cyp3A5 and the PXR genotype. Currently, in kidney transplant practice, genotyping is not routinely performed, yet. Research on pharmacogenetic testing and the potential introduction of a pharmacogenetic passport is currently being performed. ${ }^{3}$ Until then, awareness of the influences of the PXR SNP's and the Cyp3A5 SNP's on tacrolimus pharmacokinetics could help the clinician to understand inter- and intra-individual differences in tacrolimus levels implying the need for strict TDM. 


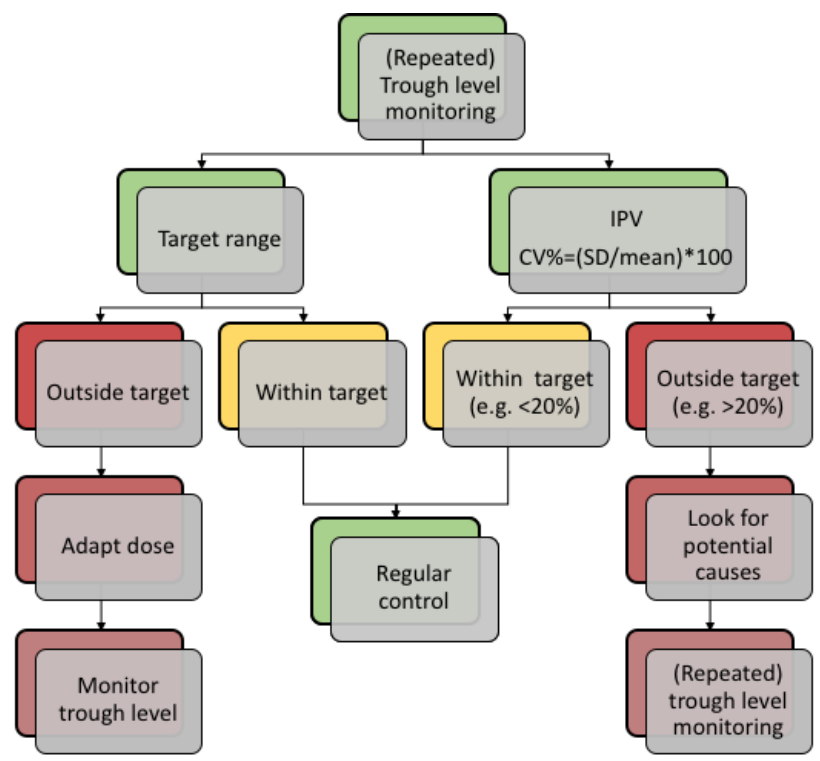

Figure 9.1 Flow chart for (repeated) trough level monitoring and usage of the IPV. CV = coefficient of variation; SD = standard deviation.

Table 9.1 Potential tools for identifying causes for high intra-patient variability of tacrolimus exposure.

\begin{tabular}{ll}
\hline Risk factor & Check for: \\
\hline Therapy adherence & Medication delivery (contact pharmacy) \\
& Correct dose \\
& Correct time \\
& Tacrolimus blood level (on demand: DBS) \\
& IPV \\
& Medication in use (contact pharmacy) \\
Drug or herbal interaction & Use of herbal products \\
& Tacrolimus blood level \\
Corticosteroids & Prescription or withdrawal of steroids \\
& Tacrolimus blood level \\
Food & Random switch of fasted vs. non-fasted intake \\
& Time of drug intake in relation to food \\
& Fat content of food \\
Generic substitution & Tacrolimus blood level \\
& Medication in use (contact pharmacy) \\
Tacrolimus assay & Tacrolimus blood level \\
& Contact laboratory: \\
Intercurrent illness & Different assay? \\
Quality control issues? & Diarrhea? \\
Liurnal variation & Low haematocrit or albumin? \\
& Liver disease? \\
& Tacrolimus blood level \\
(Switch of) Time of ingestion & Tacrolimus blood level \\
\hline
\end{tabular}




\subsection{What is innovative in this thesis?}

In Chapter 2, we have demonstrated that carriers of Cyp3A5*1 exhibit a significant lower rise in dose-normalized trough levels $\left(\mathrm{DnC}_{0}\right)$ after steroid taper compared to the Cyp3A5 *3 homozygotes and that PXR plays a role in tacrolimus pharmacokinetics. Given these new insights, the clinician could now understand why patients show different patterns of tacrolimus dose requirement after steroid withdrawal. This way, changes in tacrolimus pharmacokinetics after steroid tapering, withdrawal or after steroid prescription can be anticipated by the clinician through therapeutic drug monitoring. Also, apart from a potential reduction of cardiovascular risk, the reduced tacrolimus requirements could considerably lower costs.

In Chapter 3, a pharmacokinetic study on alternative routes of tacrolimus administration has been presented. This study was the first to deliver robust data of true sublingual and of rectal application, since firm pharmacokinetic data on this issue had not been available, so far. The findings of this study may reveal an alternative for the potentially cumbersome intravenous application of tacrolimus.

It has clearly been demonstrated that true sublingual tacrolimus absorption is absent, which is contradictory to other, clinical studies. We concluded that the levels achieved in these studies rather resulted from regular gastro-intestinal absorption. This let us also conclude that the gastro-intestinal tract is available for tacrolimus absorption, even under circumstances that this is not obvious. Therefore, normal gastro-intestinal absorption should be considered during hospitalization of the renal transplant patient, instead of putting the patient at risk by conversion to the i.v. solution.

The rectal route of absorption, on the other hand, has clearly been shown to be an alternative site of tacrolimus application. A simple suppository as used in our study could be manufactured by every hospital pharmacy. Not only did this study reveal an alternative for the potentially cumbersome intravenous application, but it also raised awareness of potential systemic (side-) effects when used as a local therapy for colitis. $^{4}$

The dosing recommendations for oral tacrolimus according to the product specifications, i.e. one hour before or two hours after a meal, is not very convenient for the patient and could also be a source for non-adherence and thus variability. No such data had been available for Tac QD in kidney transplant patients, yet. In Chapter 5 , a study has been performed to describe the influence of a continental breakfast on the bioavailability of Tac QD compared with fasted intake of tacrolimus. Similar to the former findings on the Tac BID formulation ${ }^{5,6}$, it has been demonstrated that when Tac QD was ingested in a non-fasted state, i.e. together with a standard continental breakfast, the $\mathrm{AUC}_{0-24}$ slightly decreased together with a prolongation of the $\mathrm{T}_{\max }$. Also, there was a large spread in individual changes in $\mathrm{AUC}_{0-24}$, and all but one trough level remained within the target range. Based on these findings, non-fasted tacrolimus 
intake, i.e. together with a continental breakfast, is considered to be safe and may be more convenient.

Therefore, patients from our centre are allowed to take the drug together with a continental breakfast, provided they have a stable renal function with adequate tacrolimus trough levels at about 2-3 weeks after transplantation. Given the interindividual differences in the effect of food on tacrolimus exposure, we recommend monitoring of tacrolimus blood levels at one week after conversion to sustained nonfasted intake. Future studies will have to prove if non-fasted intake of tacrolimus leads to better adherence, whether the IPV changes under non-fasted intake and whether trough level monitoring suffices for estimation of drug exposure under non-fasted intake compared with other TDM strategies (e.g. later or more frequent sampling).

We have shown in Chapter 4, that measurement of the Tac QD levels eight hours after dosing, more adequately predicts true drug exposure (AUC) than the trough level does in stable kidney transplant patients. Early after transplantation, adding samples also at two and twelve hours after dosing gave the best prediction of the tacrolimus exposure. Later and more frequent venous measurement for this would be rather inconvenient. Home-based therapy monitoring by capillary blood sampling, like the DBS method used in this thesis, can overcome these inconveniences.

Capillary blood sampling is a relatively novel tool for home measurement of tacrolimus levels. The patients themselves at home can perform it. Methods for capillary sampling are further improving with respect to patient convenience and accuracy of blood specimen analysis. ${ }^{7}$ Whilst home-based TDM by means of the DBS has already been established in our outpatient practice ${ }^{8,9}$, the DBS-method has also been used for compilations of repetitive full 24-hour blood concentration-time profiles presented in this thesis (Chapter 6). Capillary blood sampling thereby enabled this scientific research that would not otherwise have been feasible due to a substantial patient burden and costs for hospitalization.

In Chapter 6, it has been shown that Tac QD has a reduced pharmacokinetic variability of tacrolimus exposure compared with Tac BID. Given the association of worse graft survival in patients with a high IPV, tacrolimus therapy should not only consist of trough level measurement, but the clinician should also be aware of the IPV. ${ }^{10}$ It has been suggested to be an important measure to identify patients "at risk" and may deliver a tool to monitor the success of implemented interventions. ${ }^{11,12}$ In case of a high IPV, the clinician should look for potential causes, as outlined under paragraph 9.3.

Altogether, the findings presented in this thesis could result in improved (out-) patient management through patient empowerment, shared decision-making and possible implementation of an e-health concept that could help to reduce costs. 


\section{References}

1. Laupacis A, Keown P, Pus N, et al. A study of the quality of life and cost-utility of renal transplantation. Kidney Int 1996;50(1):235-242.

2. Wolfe RA, Ashby VB, Milford EL, et al. Comparison of mortality in all patients on dialysis, patients on dialysis awaiting transplantation, and recipients of a first cadaveric transplant. N Engl J Med 1999; 341(23):1725-1730.

3. van der Wouden $\mathrm{CH}$, van Rhenen $\mathrm{MH}$, Jama WOM, et al. Development of the PGx-Passport: A Panel of Actionable Germline Genetic Variants for Pre-Emptive Pharmacogenetic Testing. Clin Pharmacol Ther 2019;106(4):866-873.

4. Jaeger SU, Klag T, Hoeger K, et al. Tacrolimus Suppositories in Therapy-Resistant Ulcerative Proctitis. Inflamm Intest Dis 2019;3(3):116-124.

5. Christiaans M, van Duijnhoven E, Beysens $T$, et al. Effect of breakfast on the oral bioavailability of tacrolimus and changes in pharmacokinetics at different times posttransplant in renal transplant recipients. Transplant Proc 1998;30(4):1271-1273.

6. van Duijnhoven E, Christiaans M, Undre N, et al. The effect of breakfast on the oral bioavailability of tacrolimus in diabetic and nondiabetic patients before transplantation. Transplant Proc 1998;30(4): 1268-1270.

7. Protti M, Mandrioli R, Mercolini L. Tutorial: Volumetric absorptive microsampling (VAMS). Anal Chim Acta 2019;1046: 32-47.

8. Hoogtanders $\mathrm{K}$, van der Heijden J, Christiaans M, et al. Therapeutic drug monitoring of tacrolimus with the dried blood spot method. J Pharm Biomed Anal 2007;44(3):658-664.

9. Hoogtanders K, van der Heijden J, Christiaans M, et al. Dried blood spot measurement of tacrolimus is promising for patient monitoring. Transplantation 2007;83(2):237-238.

10. Susal C, Dohler B. Late intra-patient tacrolimus trough level variability as a major problem in kidney transplantation: A Collaborative Transplant Study Report. Am J Transplant 2019;19(10):2805-2813.

11. Knight SR. Intrapatient variability in tacrolimus exposure - a useful tool for clinical practice? Transpl Int 2016;29(11): 1155-1157.

12. Kuypers DR. Intrapatient Variability of Tacrolimus Exposure in Solid Organ Transplantation: A Novel Marker for Clinical Outcome. Clin Pharmacol Ther 2020;107(2):347-358. 
Addenda 
Dankwoord 


\section{Dankwoord}

Tsja, daar gaan we dan: een niet beoordeeld maar het meest gelezen onderwerp van een proefschrift: het dankwoord. Het dankwoord, waar in mijn geval zo menigeen lang op heeft zitten wachten...Een traject van tien jaar betekent ook dat het aantal mensen waar dank aan verschuldigd is, evenredig toeneemt. Ik hoop dan ook dat ik niemand vergeet die enige rol van betekenis heeft gespeeld bij het tot stand komen van dit boekje. Maar ja, bij een inmiddels bereikte leeftijd van bijna 50 jaar en een druk bestaan, kunnen hier en daar wel eens wat dingen vergeten worden...

Als eerste gaat mijn dank uit naar de mensen die direct betrokken zijn geweest bij het tot stand komen van het proefschrift. Dank aan mijn promotor Hans van Hooff. Toen wij aan het project promotie begonnen was jij nog volop aan het werk als hoofd van de transplantatieafdeling in het MUMC. De kans zat erin dat met een dergelijke duur voor het afronden van mijn promotie er een pensioen voor je aan zat te komen. Inmiddels is dit reeds sinds meerdere jaren het geval maar blijf je met al je enthousiasme betrokken bij de transplantatiegeneeskunde. Ook je "ius promovendi" heb je laten verlengen om dit proefschrift tot een goed einde te brengen, waarvoor mijn allergrootste dank. Ik hoop dat ik de eer die mij is toebedeeld je laatste promovendus te zijn, waardig ben.

Ook dank aan mijn co-promotor Maarten Christiaans die een bijzondere rol in mijn promotietraject heeft gespeeld. Deze rol rijkt verder dan alleen maar dit boekje. Reeds als mentor tijdens mijn 2e jaar van mijn studie geneeskunde kwam jij in beeld. Vervolgens feliciteerde jij mij als lid van de examencommissie in de Sint Janskerk met het behalen van mijn artsdiploma en was je mijn supervisor tijdens mijn opleiding tot internist en in het bijzonder tijdens mijn specialisatie tot nefroloog. Op jouw uitnodiging mocht ik mee naar het grote ATC-congres in Toronto. Onder het genot van een kop koffie met een muffin bij de Starbucks heb jij je voorstellen gedaan wat ik verder zou kunnen onderzoeken in het kader van een promotie. Dat was voor mij dus het gevoelde startschot voor dit proefschrift. Met precisie en volharding heb jij mij vervolgens geleerd hoe de data te interpreteren en hoe daar ook de relevante klinische implicaties aan toe te kennen. Inmiddels zijn wij ongeveer 21 jaar verder sinds onze eerste ontmoeting en hoop ik dat wij elkaar ook in de toekomst met enige regelmaat zullen tegenkomen.

Dear Nas Undre. For you, I had to decide in which language to acknowledge you for your distinguished contribution to my thesis. You are a real cosmopolitan and lived for quite some time in my home country. As I assume that English will be the preferred language of the readership I will continue in English. You have often visited the 
transplantation department of the MUMC and shared your pharmacokinetic viewpoint with us that eventually led to the conversion study presented in Chapter 6 . Thanks to your brainstorming participation we were able to design the conversion study to definitely clarify the unanswered question on intra-patient variability of the modified release once daily tacrolimus formulation. Besides, you had previously been involved in the phase 3 trial that led to the food sub-study which we were able to publish, as presented in Chapter 5 . Last but not least, you also kindly provided us with the raw pharmacokinetic data from the French group that let us validate a limited sampling strategy for prediction of tacrolimus exposure early after transplantation. To put it in a nutshell: your footprint is indispensable in this thesis. Thank you very much!

Een hele grote dank ook aan Sander van Kuijk, van de afdeling KEMTA. Sander, jouw methodologische blik en statistische analyse van de data uit de hoofdstukken 2 en 4 hebben essentieel ertoe bijgedragen dat de enorme hoeveelheid aan getallen en variabelen overzichtelijk en inzichtelijk werd met een boodschap. Jij wist de methodologische vertaalslag te maken van data naar kliniek en hebt dit met veel geduld en accuratesse uitgewerkt en, met name, begrijpelijk uitgelegd. Ik ben je hiervoor zeer erkentelijk.

Karin Hoogtanders en Yvo de Beer, jullie lab heeft een onmisbare bijdrage geleverd door bepaling van de Tacrolimus spiegels uit de in de totaal 3.200 (!!!!) monsters die via de post jullie lab hebben bereikt. En als de monsters via de post worden bezorgd dan weet iedere ingewijde lezer dat het hierbij gaat om de dried blood spot methode. Een methode waar jullie samen met Jac van der Heijden pionierswerk hebben verricht. Werk dat een soortgelijke studie zoals de conversie studie überhaupt mogelijk heeft gemaakt. Daarnaast ben ik jullie dankbaar voor de praktische inzichten rondom de kwaliteitscontrole van de tacrolimus bepalingen. Ik hoop dat we ons voornemen om dit proefschrift met een etentje af te sluiten, nog waar zullen maken.

Ook een grote dank voor Ptal Wijnen en Otto Bekers. De grote DNA-databank is jullie onmisbare bijdrage voor dit proefschrift. Ptal, jij hebt me mee laten kijken in je DNAkeuken waardoor iets abstracts voor mij concreet is geworden. Ik was enorm gerustgesteld dat ook jij het Hardy Weinberg disequilibrium had gezien. Simpelweg als bevestiging dat ik geen domme rekenfout had gemaakt. En Otto, uiteraard dank voor je bijdrage tot de PXR-studie met het mede publiceren van resultaten.

Monique Mullens. Het is zo ver. Het dankwoord is geschreven! Hoe het dankwoord naar jou gericht te schrijven, heeft me wel menige hoofdpijn bezorgd. De overpeinzingen begonnen al bij de vraag naar de juiste aanrede: "uwe koninklijke hoogheid", "Princes Monique" of gewoon "Monique"? En hoe je bijdrage voldoende in de juiste, in de verblindende schijnwerpers te plaatsen? 
Met enige regelmaat begon jij na bepaalde tijd reeds te informeren naar de vorderingen van het dankwoord. Deze onmiskenbare hint bleef een niet te onderschatten drijfveer om je welgemeende aanmoediging niet in de wind te slaan. Dat heb ik blijkende dit gedrukte werk ook niet gedaan en mocht ik je uiteindelijk de verlossende app sturen dat het proefschrift bij de beoordelingscommissie was beland. Bewust van de historische waarde ervan stuurde jij zowaar een kaartje om te feliciteren. lets dat onderstreept hoe betrokken jij bent geweest gedurende al die jaren. Deze betrokkenheid hield niet op met het officiële einde van de conversie- en de voedselstudie maar duurde voort tot het bittere einde. Hiermee ben jij een waardige paranimf geworden, en was het overigens al snel duidelijk dat de helft van de vacatures voor deze post aan jou vergeven zou worden.

Jouw betrokkenheid is een waarde die ook voor de patiënten herkenbaar is. Jij staat er voor ze als een rots in de branding. Jij leerde ze het prikken in de vinger, jij motiveerde de patiënten, jij bewaakte de goede gang van zaken. Deze motivatie en begeleiding toon je niet slechts in studieverband. Nee, jij bent een echte 'zuster' voor de patiënten. Ik durf te wedden dat de transplantatie patiënten uit het MUMC het mede zo goed doen dankzij jou. lets wat wij nog nooit wetenschappelijk hebben onderzocht, waarvan ik echter stellig durf te beweren dat het de toets zeker zou doorstaan (zie ook de stellingen). Monique, dank je wel voor je steun en toeverlaat.

Dit proefschrift zou uiteraard nooit tot stand zijn gekomen zonder de hoofdrolspelers waar dit boek om draait: de patiënten zelf. Dankzij hun interesse in veranderingen en medewerking is een mooie studie tot stand gekomen die tot op de dag van vandaag niet geëvenaard kon worden: een studie waar iedere patiënt wekelijks in totaal 10 opeenvolgende 24-uurs curves bij elkaar wist te prikken. Daarnaast heeft het overgrote deel ook nog hun medewerking verleend aan de voedselstudie die met een 24 uurs opname gepaard ging. Ik hoop via dit proefschrift een kleine bijdrage te kunnen leveren dat het beter met hun mag gaan.

Tiny Wouters, ook jij bent inmiddels met pensioen, maar blijf jij als een soort hobby de lay out maken van menig proefschrift. Dank dat ook ik nog toe mocht treden tot de grote club van promovendi waar jij de lay out van het boekje hebt verzorgd!

Jean Scheijen. Aan jou de eer om in de laatste fase van mijn proefschrift betrokken te worden. Jij hebt mijn persoonlijke wensen met jouw persoonlijke ideeën en artistieke creativiteit omgezet in het zichtbaarste resultaat van dit proefschrift: de kaft. Zoals voor iedere promovendus zal gelden, bevat de kaft een boodschap vol persoonlijke symboliek. lets dat evenveel over de persoon vertelt als de stellingen. Jij hebt dit tot uitdrukking weten te brengen waarvoor mijn zeer grote dank. 
Pascal en Maaike Walters. Dank voor jullie adviezen en inzet om mijn proefschrift in jullie drukkerij vorm te geven.

Binnen mijn Zuyderland MC gaat natuurlijk als eerste grote dank naar mijn collega's binnen de Co-MIK. De groep is ondertussen dermate groot dat een dankwoord aan ieder individu alleen al hierdoor het hele proefschrift zou vullen. De oorspronkelijk twee aparte maatschappen zijn namelijk samengegroeid tot één groot geheel. Teamspirit staat hoog in het vaandel en zonder dit team was dit werk niet haalbaar geweest. Een team dat gedurende mijn werk als medisch specialist successievelijk werd geleid door Frans Erdkamp, Eric Keulen, Liekele Oostenbrug en Kristy Pickwell. Zonder jullie strakke leiding en organisatie waren er geen randvoorwaarden denkbaar geweest voor dit soort exercities. Bijzondere dank ook aan Kristy, voor je tips, je morele steun en de nodige coffeïne gedurende al die jaren. Ik hoop dat de cappuccino rekening nog meevalt.

Bijzondere dank in deze aan mijn directe collega's nefrologen. Jullie zijn altijd voor mij in de bres gesprongen om ondanks alle drukte, mij de ruimte te geven om deadlines te kunnen halen, maar ook om in minder vrolijke tijden mijn moeder bij te kunnen staan. Mariëlle, die al direct in actie komt om het rooster aan te passen, en samen met Gaico en Frans nooit te beroerd om taken over te nemen. Leon, Sören (sorry voor het snurken) en Nicole (lang leve de smallo) die in deze tijden alleen het Parkstad-fort moesten verdedigen en uiteraard Karen die ons heel veel werk uit handen neemt. Liv, bijzondere dank ook naar jou. Jij hebt de roosterplanning overgenomen, waardoor er in mijn agenda meer ruimte is ontstaan. En niet te vergeten: zonder je inlogcodes was het verkrijgen van de nodige artikelen toch een stuk lastiger geweest! Ik hoop dat voor jouw promotietraject ook ik een bijdrage kan leveren dat het uiteindelijk afgerond kan worden.

En over inlogcodes gesproken: Daan, dankzij je SPSS-licentie kon ik nog de nodige grafieken van mijn laatste publicatie aanpassen en versturen! En uiteraard bijzondere dank aan Ad van Bodegraven (@je). Jij nam ondanks je multiple taken als begeleider van talloze promovendi, medeauteur van niet op te tellen publicaties en lid van menige review board de tijd, om mijn stuk door te nemen. "Beter is de vijand van goed" zou een mooie stelling zijn geweest, maar zou wellicht niet de toetsing van plagiaat hebben doorstaan. Dank Ad, en ik hoop dat je het mij als opponent niet te lastig zult maken (ik ken je wel een beetje ;-)). Guy, jou wilde ik nog bedanken als trouwe kamer- en Loipe-genoot in Sankt Christoph! Dank voor de gezelligheid en de Spass,...wa! 
Dank ook aan mij voormalige Opleider Bert-Jan Looij. Bert, jij bent een voorbeeld geweest en aan mij de eer om in jouw voetstappen te treden (in waarnemende functie). Het moge duidelijk zijn dat je voetsporen een dermate grote afdruk hebben achtergelaten, dat dit nauwelijks eervol op te volgen is. Tot de dag van vandaag haal ik ook na jouw pensioen soms nog advies bij je in. Ik hoop dat het al lang geplande etentje toch een keer doorgang kan vinden. Met het vorderen van de tijd waardeer ik steeds meer wat jij als opleider voor iedereen hebt betekend. Ik hoop dat ik vele wijze lessen van je door mag geven aan de huidige generatie arts assistenten $(A(N) I O S)$. Eén van de inzichten die ik probeer door te geven is dat wij elkaar opleiden.

Daarom wilde ik alle $\mathbf{A}(\mathbf{N})$ IOS die ik in de afgelopen jaren heb mogen leren kennen, op deze plaats bedanken. Ik bewonder jullie flexibiliteit, de snelheid waarmee jullie dingen oppakken, jullie snelheid om foutloos te typen (wat ik niet van mezelf kan beweren), jullie inspiratie en jullie inzichten. Met name dank voor de sfeer die jullie erin blijven houden, de collegialiteit en het voortzetten van het teamspel. Het Medisch Team Covid-19 toont waartoe jullie in staat zijn in deze historische tijden van de SARS CoV2 pandemie. Nogmaals, grote dank hiervoor.

Ook opleiden doe je niet alleen. Hiervoor hebben we een heel team geformeerd met Jacqueline en Roderick in de voorste gelederen. Maar aan het gehele CoMIK-eigen opleidings-DB ben ik grote dank verschuldigd dat wij de taken kunnen verdelen. Taken waar onze Annette Marneef voor zorgt, dat alles in goede banen verloopt.

Naast mijn werk-familie, gaat uiteraard ook grote dank uit naar mijn naaste familieleden en vrienden. Mama. Jij hebt het niet gemakkelijk gehad. Toen ik zou gaan studeren heb jij alles op alles gezet om dit voor mij mogelijk te maken. En ook al haalde je zoonlief het in zijn hoofd om nog maar eens een studie er achteraan te plakken; jij steunde mijn beslissingen. Deze steun was onvoorwaardelijk en dierbaar. Alleen hiervoor al reden genoeg om ieder begonnen traject tot een goed einde te brengen. Zo ook dit boekje. Helaas mag je dit niet meer meemaken, maar ik weet hoe apetrots je zou zijn geweest. Zo trots dat ik je waarschijnlijk een beetje had moeten temperen. Samen met Gerard had jij mij door je aanwezigheid gedurende het uur tot het verlossende 'hora est' willen geruststellen. Je kunt je niet voorstellen hoe zeer ik het mis dat zich deze gelegenheid niet voordoet. Maar ik weet dat je erbij bent. Dank je wel Mama. Ook dank aan Gerard. Jij week nooit van de zijde van Mama. Samen hebben jullie mij in alles gesteund en mij naar iedere uithoek in Duitsland gebracht, zodat ik bij iedere belangrijke wedstrijd aan de start kon verschijnen. Jij maakte deel uit van deze voor mij zeer belangrijke levensfase en dank dat jij nog steeds een lieve Opa bent voor mijn kinderen. 
Vater. Nach all den Jahren ist dieses nun der vorläufige Höhepunkt meiner bis dahin für dich wahrscheinlich nicht immer durchsichtigen Karriere. Eine Karriere, die sich über Jahrzehnte dahingezogen hat, in denen du mich mitgeprägt hast. Vom Wisseler See und dem Readymix Betonmischer, über die Liebe zur Natur und zu den Bergen, bis zum Autoverleih des "Red Porsche Killers", der mir die notwendige Mobilität in meinem abenteuerlichen Leben verlieh. Auch du hast versucht, mich zu unterstützen, wo es im Rahmen der Möglichkeiten ging. Danke dafür und auch Dank an alle meine Familienmitglieder, die mich mit ihren Gratulationen und Stolzbekundungen sehr glücklich gemacht haben.

William, Jan en Henk (R.I.P.). Dank aan eenieder van jullie dat jullie in al die jaren voor mij voorbeelden hebben mogen zijn. William, die grapjes met mij maakte toen ik nog klein was ("Willam drauf";-)). Later met Mirjam overal met mij op pad, mij deel hebben laten uitmaken in de wereld van de sport. Jan, jij hebt laten zien hoe ver men het kan schoppen met discipline om hard te studeren, ook al zijn de omstandigheden niet optimaal. Jammer dat je eigen toestand het niet meer toelaat om mijn promotie bij te wonen. Ik weet dat je trots zou zijn. Henk, helaas ben ook jij veel te vroeg van ons heen gegaan. Maar ook jij hebt samen met Diane een belangrijk deel van mijn leven vormgegeven, met je aanstekelijk humor en de onvergetelijke, gezellige BBQ's in de Vogelweide. Mogen wij deze banden en tradities met Diederik, Roeland, Bram en Nik in ere houden.

Dank aan mijn schoonouders, Jack en Leny die altijd klaar stonden om onze kinderen op te vangen. Jack, ook bedankt voor al die klussen die je toch altijd weer rond ons huis weet te klaren. Bijzondere dank ook aan Michelle, voor je feedback en het meedenken bij de stellingen. Thanks also to Danny Rozen, for your empathy and for sharing your knowledge. Dank ook aan Laurent en Liza, Kelly en Piet voor de gezellige verjaardagen, Sinterklaasavonden en de weekenden samen in Center Parcs. Jullie zorgden allemaal voor de nodige afleiding. Piet, ook dank voor je knowhow en je speurwerk als er weer eens wat mankeert aan de auto!

Jens. Mensch, wer hätte es sich ausdenken können, dass du 28 Jahre nach unserem ersten gemeinsamen Trainingslager, als die Medizin nichts Weiteres war als ein intensives Gesprächsthema, mir nun am Tag meiner Dissertation beistehen würdest? Die legendäre Schicht auf der Intensivstation in Essen, hatte mich damals definitiv infiziert und mich den Weg des Medizinstudiums einschlagen lassen. In der Zwischenzeit ist viel in unserem Leben passiert, und schließt sich der Kreis. Eine Freundschaft, über die man ein Buch schreiben könnte. Danke, dass du immer für mich zur Stelle warst, danke für all die gemeinsamen Trainingsstunden, Bergtouren und Loipenkilometer in Leutasch! Möge uns dies noch weiterhin gelingen! 
Und wo man von Prägung und Freundschaften spricht, dürfen meine Freunde des ersten Triathlon Teams in der Geschichte der SSV Hellas Emmerich nicht fehlen, Ulrich, Guido, Klaus und Markus. Wir haben etwas aufgebaut, was uns keiner so leicht nachmacht. Nebst unseren Vereinsrekorden, werden auch unsere gemeinsamen Abenteuer als „Windeier" in den Geschichtsbüchern vermerkt bleiben. 30 Jahre nach unserem ersten gemeinsamen Rennen haben wir vorgemacht, was Durchhaltevermögen, Mannschaftsgeist und Freundschaft bedeutet. Eigenschaften, ohne die ich diese Doktorarbeit niemals vollbracht hätte.

Lothar und Nicole. Gemeinsam im C-Kader, zusammen am Start in Montluçon und in Orlando, zu sechst im Volvo von Embrun nach Darmstadt und hoffentlich (wenn es die Corona Krise zulässt), nach 30 Jahren, die erste Passhelden Etappe ab Girona. Ich glaube es bedarf keiner weiteren Worte, um auszudrücken, wie dankbar ich bin, dass sich unsere Wege gekreuzt haben. Danke für die alljährlichen Aktionen und ab und an ein Weinfest ;-). Die Gedanken an unsere gemeinsamen Abenteuer verleihen mir stetig Rückhalt.

Natürlich darf mein alter Trainer und Freund Peter (Piotr) nicht unerwähnt bleiben. $\mathrm{Du}$ hast mich auf den Höhepunkt meiner sportlichen Karriere gebracht. Dabei allerdings so manchen Tropfen Blut aus meinem Ohrläppchen gemolken, der nicht nur der Trainingssteuerung, sondern auch der Datensammlung deiner damals anvisierten Doktorarbeit dienen sollten. So manchen Witz haben wir damals darüber gerissen, habe jetzt aber eigens erfahren, dass eine Doktorarbeit nicht immer ein Honigschlecken ist und dem Hochleistungssport ähnelt. Dank für deinen unermüdlichen Einsatz und der fortwährenden Freundschaft.

Prof.Dr.rer.nat. Norbert Maassen. Norbert, auch du hast mich geprägt. Während meiner Zeit in deinem Labor an der Medizinischen Hochschule Hannover habe ich gelernt, was es bedeutet, akribisch Daten zu verarbeiten und kritisch zu interpretieren. Voraussetzungen, die mir sicherlich bei der vorliegenden Doktorarbeit von Nutzen waren. Ich hoffe, dass wir uns nochmals zu gegebenem Anlass begegnen werden. Bis dahin lautet unser Motto: „Laktat macht dumm“.

Robin und Katrin: dank Eurer Freundschaft habe ich eine wunderbare Zeit in Hannover verbringen können. Gemeinsame Trainingsstunden rundum „Harnblasen“, sprachwitzige Unterhaltungen bis sich die Balken biegen und wortgewandte Italiener, die ihren Urlaub in Malta verbringen, um nur einige Kapitel meiner Autobiographie zu erwähnen. Robin hat vorgelegt mit seiner Doktorarbeit, da musste ich nachziehen. Danke, dass es euch gibt. 
Mijn studie- en sportvrienden Nico, Armand, Aart, Robert, Jochen, Gerben, Mark en Casper. De wereld van een triatleet in de jaren 90 was klein en het was geen toeval dat wij elkaar zouden vinden met onze gemeenschappelijke achtergrond. Ook wij gaan samen door dik en dun, steunen elkaar, hebben een kritische kijk op de (medische) wereld en motiveren elkaar in onze academische loopbanen. Dank jongens.

Dank aan al onze buren in Wolder en in het bijzonder Piet en Florence. Jullie zijn er altijd om ons uit de brand te helpen. Jullie zijn onze redding met de reservesleutel, jullie letten op onze Grummelke en letten op ons huis. ledereen in onze buurt toont interesse voor elkaar en jullie hoorden ook geduldig de eerste versie van mijn lekenpraatje aan (in de hoop dat alles doorgang mag vinden). Mijn dank hiervoor.

Audrey, du bist ein wunderbarer Mensch. Dein Humor, deine fröhliche Natur, deine Ausstrahlung, deine Musikalität haben mich verzaubert. Du bist die beste Mutter, die ich mir für unsere Kinder vorstellen kann. In den vergangenen 10 Jahren war das Leben bei uns zu Hause leider viel geprägt von Arbeit und so manchem Stress. Es wird nicht immer leicht gewesen sein, meinen Unmut zu ertragen. Dennoch hast du zu mir gehalten. Danke. Ich hoffe, dass mit dieser Arbeit ein wichtiges Kapitel abgeschlossen werden kann und ich etwas entspannter das Leben mit Dir und den Kindern genießen werde. Lasse uns die Zukunft gemeinsam so positiv gestalten, wie du bist.

Yana und Laura. Ihr seid inzwischen groß geworden und werdet bald auf eigenen Beinen stehen. Dennoch hoffe ich, trotz allen Stresses, ein guter Vater für euch gewesen zu sein. Ihr habt mich bisweilen das Leben relativieren lassen, mich zurück auf den Boden der Tatsachen gebracht. Yana, deine Leidenschaften zum Fußball, deine Reiselust, deinen Einsatz für dein Studium, dein Zeichentalent und Laura, deine Leidenschaft für Jolly und dein Ehrgeiz beim Lernen erfüllen mich mit Stolz und Liebe. Eure Liebe auch für unser zusätzliches Familienmitglied Grummelke zeigt, wie groß eure Herzen sind. Mögen diese Herzen lange klopfen und nicht gebrochen werden. Ich möchte auch für euch in der Zukunft zusammen mit Mamma ein Rückhalt sein. Ich bin unglaublich stolz auf euch, habe euch ganz doll lieb!

Met andere woorden: promoveren doe je niet alleen. Getrouw het motto in deze historische Covid-19 dagen: You never walk alone (however, you better should, in these days). 
List of publications 



\section{List of publications}

1. Dammers, R., F. Stifft, J. H. Tordoir, et al. (2003). Shear stress depends on vascular territory: comparison between common carotid and brachial artery. J Appl Physiol (1985) 94(2): 485-489.

2. Stifft, F., R. Dammers, J. Tordoir, et al. (2003). [Real-time registration of flowmediated dilatation for the assessment of endothelial function]. Ultraschall Med 24(1): 34-39.

3. Stifft, F., L. M. Stolk, N. Undre, et al. (2014). Lower variability in 24-hour exposure during once-daily compared to twice-daily tacrolimus formulation in kidney transplantation. Transplantation 97(7): 775-780.

4. Stifft, F., F. Vanmolkot, I. Scheffers, et al. (2014). Rectal and sublingual administration of tacrolimus: a single-dose pharmacokinetic study in healthy volunteers. Br J Clin Pharmacol 78(5): 996-1004.

5. Stifft, F., N. Undre, J. P. van Hooff, et al. (2016). Effect of Breakfast on the Exposure of the Once-Daily Tacrolimus Formulation in Stable Kidney Transplant Recipients. Ther Drug Monit 38(4): 456-462.

6. Timmermans, S. A., M. H. Christiaans, M. A. Abdul-Hamid, et al. (2016). Granulomatous interstitial nephritis and Crohn's disease. Clin Kidney J 9(4): 556559.

7. Broers, N. J. H., R. J. H. Martens, T. Cornelis, et al. (2017). Physical Activity in EndStage Renal Disease Patients: The Effects of Starting Dialysis in the First 6 Months after the Transition Period. Nephron 137(1): 47-56.

8. Broers, N. J. H., R. J. H. Martens, B. Canaud, et al. (2018). Health-related quality of life in end-stage renal disease patients: the effects of starting dialysis in the first year after the transition period. Int Urol Nephrol 50(6): 1131-1142.

9. Stifft, F., S. M. J. van Kuijk, O. Bekers, et al. (2018). Increase in tacrolimus exposure after steroid tapering is influenced by CYP3A5 and pregnane $\mathrm{X}$ receptor genetic polymorphisms in renal transplant recipients. Nephrol Dial Transplant 33(9): 1668-1675.

10. Gennip, A., N. J. H. Broers, K. J. T. Meulen, et al. (2019). Endothelial dysfunction and low-grade inflammation in the transition to renal replacement therapy. PLoS One 14(9): e0222547.

11. Martens, R. J. H., N. J. H. Broers, B. Canaud, et al. (2019). Relations of advanced glycation endproducts and dicarbonyls with endothelial dysfunction and lowgrade inflammation in individuals with end-stage renal disease in the transition to renal replacement therapy: A cross-sectional observational study. PLoS One 14(8): e0221058. 
12. Stifft F, Vandermeer F, Neef C, van Kuijk S, Christiaans MHL (2020) A limited sampling strategy to estimate exposure of once-daily modified release tacrolimus in renal transplant recipients using linear regression analysis and comparison with Bayesian population pharmacokinetics in different cohorts. European journal of clinical pharmacology DOI 10.1007/s00228-019-02814-x. 
Curriculum vitae 



\section{Curriculum vitae}

Frank Stifft werd geboren op 14 augustus 1970 te Goch, Duitsland. Na het behalen van het 'Abitur' (vergelijkbaar met het Nederlandse VWO-examen) aan het Städtische Hansa Gymnasium te Emmerich (Duitsland) studeerde hij vanaf 1989 aan de Rijkshogeschool Limburg, Opleiding tot Tolk-Vertaler in Maastricht.

In 1993 studeerde hij af in de vreemde talen Nederlands en Engels voor de afstudeerrichting Medicijnen en Biologie. Aanvankelijk met de bedoeling om de vakinhoudelijke medisch-biologische kennis te verdiepen begon hij in hetzelfde jaar met de studie Gezondheidswetenschappen aan de toenmalige Rijksuniversiteit Limburg, later Universiteit Maastricht.

Al snel bleek zijn voorkeur voor de bio-medische wetenschappen. Nadat hij in 1997 eerst het vereiste VWO-diploma scheikunde had gehaald, startte hij in dat jaar nog tijdens de studie Gezondheidswetenschappen ook met de studie Geneeskunde. Dit combineerde hij gedurende de eerste twee jaar met de Gezondheidswetenschappen. In 1999 behaalde hij met zijn afstudeerscriptie 'The Influence of Acute Normobaric Hypoxia on Blood Lactate Concentration during Exercise at Medium Intensity with Small and Large Muscle Groups' zijn Master's degree of Health Sciences voor de afstudeerrichting Bewegingswetenschappen onder Prof. Dr. Harm Kuipers.

In juli 2003 behaalde Frank het artsexamen (cum laude) en begon in diezelfde maand als arts-assistent niet in opleiding bij de Interne Geneeskunde in het destijds nog Atrium Medisch Centrum, te Heerlen onder dr. Paul Voogt. In november 2003 startte hij met zijn opleiding tot Internist. De eerste twee jaar aan het Academische Ziekenhuis Maastricht onder prof. dr. Karel Leunissen. Tijdens jaar 3 en 4 van zijn opleiding was Frank werkzaam in het voormalige Maaslandziekenhuis te Sittard, opgeleid door dr. Bert Jan Looij. Na terugkeer naar het Maastrichts Universitair Medisch Centrum (MUMC) onder de opleiders prof. dr. Coen Stehouwer en prof. dr. Richard Koopmans begon hij in 2007 aan het aandachtsgebied voor nefrologie onder prof. dr. Karel Leunissen en rondde op 31 januari 2010 zijn opleiding tot medisch specialist af.

Sinds 1 februari 2010 is hij werkzaam als Internist-Nefroloog aan het huidige Zuyderland Medisch Centrum (voormalig Orbis Medisch Centrum) te Sittard-Geleen en Heerlen. Vanaf diezelfde datum verrichtte hij ook het wetenschappelijk onderzoek dat tot het huidige proefschrift heeft geleid.

Frank woont sinds een groot deel van zijn curriculum vitae in Maastricht samen met Audrey Habets. Zij hebben twee dochters, Yana en Laura, die inmiddels al 19 resp. 17 jaar oud zijn. In de vrije tijd oefent hij graag zijn hobbies in verschillende takken van sport uit. 
